\title{
Biologics for chronic rhinosinusitis (Review)
}

Chong LY, Piromchai P, Sharp S, Snidvongs K, Philpott C, Hopkins C, Burton MJ

Chong LY, Piromchai P, Sharp S, Snidvongs K, Philpott C, Hopkins C, Burton MJ.

Biologics for chronic rhinosinusitis.

Cochrane Database of Systematic Reviews 2020, Issue 2. Art. No.: CD013513.

DOI: 10.1002/14651858.CD013513.pub2.

www.cochranelibrary.com 
TABLE OF CONTENTS

HEADER

ABSTRACT

PLAIN LANGUAGE SUMMARY

SUMMARY OF FINDINGS

BACKGROUND

OBJECTIVES

METHODS

RESULTS

Figure 1.

Figure 2.

Figure 3.

DISCUSSION

AUTHORS' CONCLUSIONS

ACKNOWLEDGEMENTS

REFERENCES

CHARACTERISTICS OF STUDIES

DATA AND ANALYSES

Analysis 1.1. Comparison 1 Anti-IL-4Ra mAb (dupilumab) versus placebo (on top of topical steroids), Outcome $1 \mathrm{HRQL}$ - diseasespecific (SNOT-22, 0 to 110 , lower = better).

Analysis 1.2. Comparison 1 Anti-IL-4Ra mAb (dupilumab) versus placebo (on top of topical steroids), Outcome 2 Disease severity - VAS (0 to 10, lower = better).

Analysis 1.3. Comparison 1 Anti-IL-4Ra mAb (dupilumab) versus placebo (on top of topical steroids), Outcome 3 Serious adverse events.

Analysis 1.4. Comparison 1 Anti-IL-4Ra mAb (dupilumab) versus placebo (on top of topical steroids), Outcome 4 Avoidance of surgery - number of patients who had surgery as rescue treatment.

Analysis 1.5. Comparison 1 Anti-IL-4Ra mAb (dupilumab) versus placebo (on top of topical steroids), Outcome 5 Extent of disease - endoscopy ('nasal polyps score', 0 to 8 , higher = worse).

Analysis 1.6. Comparison 1 Anti-IL-4Ra mAb (dupilumab) versus placebo (on top of topical steroids), Outcome 6 Extent of disease - CT scan (Lund Mackay, 0 to 24, higher = worse).

Analysis 1.7. Comparison 1 Anti-IL-4Ra mAb (dupilumab) versus placebo (on top of topical steroids), Outcome 7 HRQL - generic (EQ-5D VAS, 0 to 100, higher = better).

Analysis 1.8. Comparison 1 Anti-IL-4Ra mAb (dupilumab) versus placebo (on top of topical steroids), Outcome 8 Adverse events - nasopharyngitis, including sore throat (longest available data).

Analysis 2.1. Comparison 2 Anti-IL-5 mAb (mepolizumab) versus placebo (on top of topical steroids), Outcome 1 HRQL - SNOT-22 (1 to 100 , lower $=$ better) up to 25 weeks.

Analysis 2.2. Comparison 2 Anti-IL-5 mAb (mepolizumab) versus placebo (on top of topical steroids), Outcome 2 Disease severity - VAS (0 to 10 , lower = better).

Analysis 2.3. Comparison 2 Anti-IL-5 mAb (mepolizumab) versus placebo (on top of topical steroids), Outcome 3 Severe adverse events.

Analysis 2.4. Comparison 2 Anti-IL-5 mAb (mepolizumab) versus placebo (on top of topical steroids), Outcome 4 Avoidance of surgery - patients no longer meeting criteria for surgery at end of follow-up.

Analysis 2.5. Comparison 2 Anti-IL-5 mAb (mepolizumab) versus placebo (on top of topical steroids), Outcome 5 Extent of disease - endoscopic score.

Analysis 2.6. Comparison 2 Anti-IL-5 mAb (mepolizumab) versus placebo (on top of topical steroids), Outcome 6 HRQL - generic measured using EQ-5D VAS (range 0 to 100; 0 = worst, 100 = best imaginable health state) at week 25.

Analysis 2.7. Comparison 2 Anti-IL-5 mAb (mepolizumab) versus placebo (on top of topical steroids), Outcome 7 Adverse events - nasopharyngitis, including sore throat.

Analysis 3.1. Comparison 3 Anti-IgE mAb (omalizumab) versus placebo (on top of topical steroids), Outcome 1 Severe adverse events.

Analysis 3.2. Comparison 3 Anti-IgE mAb (omalizumab) versus placebo (on top of topical steroids), Outcome 2 Extent of disease - endoscopic score.

Analysis 3.3. Comparison 3 Anti-IgE mAb (omalizumab) versus placebo (on top of topical steroids), Outcome 3 Extent of disease - CT scan (lower score = better). 
Analysis 3.4. Comparison 3 Anti-IgE mAb (omalizumab) versus placebo (on top of topical steroids), Outcome 4 Adverse events

- nasopharyngitis, including sore throat.

ADDITIONAL TABLES

APPENDICES

CONTRIBUTIONS OF AUTHORS

110

DECLARATIONS OF INTEREST

110

SOURCES OF SUPPORT

111

DIFFERENCES BETWEEN PROTOCOL AND REVIEW 
[Intervention Review]

\section{Biologics for chronic rhinosinusitis}

Lee-Yee Chong ${ }^{1}$, Patorn Piromchai ${ }^{2}$, Steve Sharp ${ }^{3}$, Kornkiat Snidvongs ${ }^{4}$, Carl Philpott ${ }^{5}$, Claire Hopkins 6 , Martin J Burton ${ }^{7}$

1UK Cochrane Centre, Oxford, UK. 2Department of Otorhinolaryngology, Faculty of Medicine, Khon Kaen University, Khon Kaen, Thailand. ${ }^{3}$ National Institute for Health and Care Excellence, Manchester, UK. ${ }^{4}$ Department of Otolaryngology, Faculty of Medicine, Chulalongkorn University, Bangkok, Thailand. ${ }^{5}$ Department of Medicine, Norwich Medical School, University of East Anglia, Norwich, UK. 6ENT Department, Guy's Hospital, London, UK. ${ }^{7}$ Cochrane UK, Oxford, UK

Contact address: Martin J Burton, Cochrane UK, Summertown Pavilion, 18 - 24 Middle Way, Oxford, OX2 7LG, UK. martin.burton@cochrane.nhs.uk.

Editorial group: Cochrane ENT Group.

Publication status and date: New, published in Issue 2, 2020.

Citation: Chong LY, Piromchai P, Sharp S, Snidvongs K, Philpott C, Hopkins C, Burton MJ. Biologics for chronic rhinosinusitis. Cochrane Database of Systematic Reviews 2020, Issue 2. Art. No.: CD013513. DOI: 10.1002/14651858.CD013513.pub2.

Copyright ( 2020 The Cochrane Collaboration. Published by John Wiley \& Sons, Ltd.

\section{A B S T R A C T}

\section{Background}

This living systematic review is one of several Cochrane Reviews evaluating the medical management of patients with chronic rhinosinusitis.

Chronic rhinosinusitis is common. It is characterised by inflammation of the nasal and sinus linings, nasal blockage, rhinorrhoea, facial pressure/pain and loss of sense of smell. It occurs with or without nasal polyps.

'Biologics' are medicinal products produced by a biological process. Monoclonal antibodies are one type, already evaluated in related inflammatory conditions (e.g. asthma and atopic dermatitis).

\section{Objectives}

To assess the effects of biologics for the treatment of chronic rhinosinusitis.

\section{Search methods}

The Cochrane ENT Information Specialist searched the Cochrane ENT Register; CENTRAL (2019, Issue 9); Ovid MEDLINE; Ovid Embase; Web of Science; ClinicalTrials.gov; ICTRP and additional sources for published and unpublished trials. The date of the search was 16 September 2019.

\section{Selection criteria}

Randomised controlled trials (RCTs) with at least three months follow-up comparing biologics (currently, monoclonal antibodies) against placebo/no treatment in patients with chronic rhinosinusitis.

\section{Data collection and analysis}

We used standard Cochrane methodological procedures. Our primary outcomes were disease-specific health-related quality of life (HRQL), disease severity and serious adverse events (SAEs). The secondary outcomes were avoidance of surgery, extent of disease (measured by endoscopic or computerised tomography (CT) score), generic HRQL and adverse events (nasopharyngitis, including sore throat). We used GRADE to assess the certainty of the evidence for each outcome. 


\section{Main results}

We included eight RCTs. Of 986 adult participants, 984 had severe chronic rhinosinusitis with nasal polyps; $43 \%$ to $100 \%$ of participants also had asthma. Three biologics, with different targets, were evaluated: dupilumab, mepolizumab and omalizumab. All the studies were sponsored or supported by industry.

Anti-IL-4R $\alpha$ mAb (dupilumab) versusplacebo/no treatment (all receiving intranasal steroids)

Three studies (784 participants) evaluated dupilumab.

Disease-specific HRQL was measured with the SNOT-22 (score 0 to 110; minimal clinically important difference (MCID) 8.9 points). At 24 weeks, the SNOT-22 score was 19.61 points lower (better) in participants receiving dupilumab (mean difference (MD) - $19.61,95 \%$ confidence interval (Cl) -22.54 to -16.69; 3 studies; 784 participants; high certainty).

Symptom severity measured on a 0 - to 10 -point visual analogue scale (VAS) was 3.00 lower in those receiving dupilumab (95\% $\mathrm{Cl}-3.47$ to -2.53; 3 studies; 784 participants; moderate certainty).

The risk of serious adverse events may be lower in the dupilumab group (risk ratio (RR) $0.45,95 \%$ Cl 0.28 to $0.75 ; 3$ studies; 782 participants; low certainty).

The number of participants requiring nasal polyp surgery (actual or planned) during the treatment period is probably lower in those receiving dupilumab (RR $0.17,95 \% \mathrm{Cl} 0.05$ to $0.52 ; 2$ studies; 725 participants; moderate certainty).

Change in the extent of disease using the Lund Mackay computerised tomography (CT) score ( 0 to 24 , higher $=$ worse) was -7.00 ( $95 \% \mathrm{Cl}$ -9.61 to $-4.39 ; 3$ studies; 784 participants; high certainty), a large effect favouring the dupilumab group.

The EQ-5D visual analogue scale (0 to 100, higher = better; MCID 8 points) was used to measure change in generic quality of life. The mean difference favouring dupilumab was 8.59 ( $95 \% \mathrm{Cl} 5.31$ to 11.86; 2 studies; 706 participants; moderate certainty).

There may be little or no difference in the risk of nasopharyngitis (RR $0.95,95 \% \mathrm{Cl} 0.72$ to $1.25 ; 3$ studies; 783 participants; low certainty).

\section{Anti-IL-5 mAb (mepolizumab) versusplacebo/no treatment (all receiving intranasal steroids)}

Two studies (137 participants) evaluated mepolizumab.

Disease-specific HRQL measured with the SNOT-22 at 25 weeks was 13.26 points lower (better) in participants receiving mepolizumab (95\% Cl -22.08 to $-4.44 ; 1$ study; 105 participants; low certainty; MCID 8.9).

It is very uncertain whether there is a difference in s ymptom severity: on a 0 - to 10-point VAS symptom severity was -2.03 lower in those receiving mepolizumab $(95 \% \mathrm{Cl}-3.65$ to $-0.41 ; 1$ study; 72 participants; very low certainty).

It is very uncertain if there is difference in the risk of serious adverse events (RR 1.57, 95\% Cl 0.07 to $35.46 ; 2$ studies; 135 participants, very low certainty).

It is very uncertain whether or not the overall risk that patients still need surgery at trial end is lower in the mepolizumab group (RR 0.78 , $95 \% \mathrm{Cl} 0.64$ to $0.94 ; 2$ studies; 135 participants; very low certainty).

It is very uncertain whether mepolizumab reduces the extent of disease as measured by endoscopic nasal polyps score (scale range 0 to 8). The mean difference was 1.23 points lower in the mepolizumab group (MD $-1.23,95 \%-1.79$ to $-0.68 ; 2$ studies; 137 participants; very low certainty).

The difference in generic quality of life (EQ-5D) was 5.68 (95\% Cl -1.18 to $12.54 ; 1$ study; 105 participants; low certainty), favouring the mepolizumab group. This difference is smaller than the MCID of 8 points.

There may be little or no difference in the risk of nasopharyngitis (RR $0.73,95 \% 0.36$ to $1.47 ; 2$ studies; 135 participants; low certainty).

\section{Anti-IgE mAb (omalizumab) versus placebo/no treatment (all receiving intranasal steroids)}

Three very small studies (65 participants) evaluated omalizumab. We are very uncertain about the effect of omalizumab on disease-specific HRQL, severe adverse events, extent of disease (CT scan scores), generic HRQL and adverse effects.

\section{Authors' conclusions}

In adults with severe chronic rhinosinusitis and nasal polyps, using regular topical nasal steroids, dupilumab improves disease-specific HRQL compared to placebo, and reduces the extent of the disease as measured on a CT scan. It probably also improves symptoms and generic HRQL and there is no evidence of an increased risk of serious adverse events. It may reduce the need for further surgery. There may be little or no difference in the risk of nasopharyngitis. 
In similar patients, mepolizumab may improve both disease-specific and generic HRQL. It is uncertain whether it reduces the need for surgery or improves nasal polyp scores. There may be little or no difference in the risk of nasopharyngitis. It is uncertain if there is a difference in symptom severity and the risk of serious adverse events.

We are uncertain about the effects of omalizumab.

\section{PLAIN LANGUAGE SUMMARY}

\section{Biologics for people with chronic rhinosinusitis}

\section{What is the aim of this review?}

'Biologics' is the name given to a new type of drug. This type is increasingly being used to help people with diseases due to inflammation of body tissues. The aim of this review is to see if any of these drugs are effective in treating people with 'chronic rhinosinusitis'. These patients have long-term problems with inflammation of the nose and sinuses. This leads to them having blocked, stuffy, runny noses and pain in their cheeks. They often need to use long-term steroid nasal sprays. Some patients with chronic rhinosinusitis also get polyps in their nose. These can make their symptoms worse.

\section{Key message}

One of the new biologics - called dupilumab - helps people with severe chronic rhinosinusitis who also have nasal polyps. It makes their symptoms better and shrinks their polyps. It does not seem to cause any severe side effects. Another similar drug - called mepolizumab - may do the same but we are less certain about that.

\section{What was studied in the review?}

We looked for trials where patients with chronic rhinosinusitis had been given either one of the new biologic drugs or a placebo (dummy) treatment. They needed to have been treated for at least three months. We looked for studies that measured the effect of the drug on people's symptoms and their general health.

\section{What are the main results of the review?}

Almost all the people studied in the trials had severe chronic rhinosinusitis with nasal polyps (so we can only draw conclusions about the effects of the drugs on people like this). We found eight studies, looking at three different drugs. Most of the information we have comes from two big trials (with nearly 800 patients) looking at the effect of one drug - dupilumab.

\section{Effect of dupilumab}

After 24 weeks of treatment, people taking dupilumab have a better quality of life than those who do not and their polyps have shrunk more. On average their symptoms are probably better too, and they do not have more severe side effects than those taking placebo.

\section{Effect of mepolizumab}

The effect of mepolizumab was studied in far fewer patients and so we are less certain about the results. We can say that this drug may have similar effects to dupilumab.

\section{Effect of omalizumab}

We found very little information about the use of this drug and cannot say whether it is effective or not.

\section{How up-to-date is this review?}

The evidence is up-to-date to September 2019. 
SUMMARY OF FINDINGS

Summary of findings for the main comparison. Anti-IL-4Ra mAb (dupilumab) compared to placebo (on top of topical steroids) for chronic rhinosinusitis

Anti-IL-4Ra mAb (dupilumab) compared to placebo (on top of topical steroids) for chronic rhinosinusitis

Patients or population: patients with severe chronic rhinosinusitis with nasal polyps

Setting: tertiary care

Intervention: anti-IL-4Ra mAb (dupilumab)

Comparison: placebo (on top of topical steroids)

\begin{tabular}{|c|c|c|c|c|c|c|c|}
\hline \multirow[t]{2}{*}{ Outcomes } & \multirow{2}{*}{$\begin{array}{l}\text { Number } \\
\text { of par- } \\
\text { ticipants } \\
\text { (studies) }\end{array}$} & \multirow{2}{*}{$\begin{array}{l}\text { Relative ef- } \\
\text { fect } \\
(95 \% \mathrm{Cl})\end{array}$} & \multicolumn{3}{|c|}{ Anticipated absolute effects ${ }^{\star}(95 \% \mathrm{Cl})$} & \multirow{2}{*}{$\begin{array}{l}\text { Certainty } \\
\text { of the evi- } \\
\text { dence } \\
\text { (GRADE) }\end{array}$} & \multirow[t]{2}{*}{ What happens } \\
\hline & & & $\begin{array}{l}\text { Without an- } \\
\text { ti-IL-4Ra mAb } \\
\text { (dupilumab) }\end{array}$ & $\begin{array}{l}\text { With an- } \\
\text { ti-IL-4Ra } \\
\text { mAb } \\
\text { (dupilum- } \\
\text { ab) }\end{array}$ & Difference & & \\
\hline $\begin{array}{l}\text { Health-related quality of life } \\
\text { - disease-specific (SNOT-22, } \\
\text { range } 0 \text { to } 110 \text {, lower = better) } \\
\text { Follow-up (range): } 16 \text { to } 52 \\
\text { weeks }\end{array}$ & $\begin{array}{l}784 \\
\text { (3 RCTs) }\end{array}$ & - & $\begin{array}{l}\text { The median } \\
\text { disease-speci- } \\
\text { fic health-related } \\
\text { quality of life score } \\
\text { without anti-IL-4Ra } \\
\text { mAb (dupilumab) } \\
\text { was } 40.5 \text { points }\end{array}$ & - & $\begin{array}{l}\text { MD } 19.61 \\
\text { points lower } \\
\text { ( } 22.54 \text { low- } \\
\text { er to } 16.69 \\
\text { lower) }\end{array}$ & $\begin{array}{l}\oplus \oplus \oplus \oplus \\
\mathrm{HIGH}\end{array}$ & $\begin{array}{l}\text { At up to } 24 \text { weeks, aspects of } \\
\text { health-related quality of life that } \\
\text { are directly impacted by chronic } \\
\text { rhinosinusitis were better in par- } \\
\text { ticipants who received dupilumab. } \\
\text { The size of the difference is clinical- } \\
\text { ly significant. }\end{array}$ \\
\hline $\begin{array}{l}\text { Disease severity - VAS (range } 0 \\
\text { to } 10 \text {, lower = better) } \\
\text { Follow-up (range): } 16 \text { to } 52 \\
\text { weeks }\end{array}$ & $\begin{array}{l}784 \\
\text { (3 RCTs) }\end{array}$ & - & $\begin{array}{l}\text { The median dis- } \\
\text { ease severity score } \\
\text { without anti-IL-4Ra } \\
\text { mAb (dupilumab) } \\
\text { was - } 1.3 \text { points }\end{array}$ & - & $\begin{array}{l}\text { MD } 3 \text { points } \\
\text { lower } \\
\text { ( } 3.47 \text { lower } \\
\text { to } 2.53 \text { low- } \\
\text { er) }\end{array}$ & $\begin{array}{l}\oplus \oplus \oplus \ominus \\
\text { MODER- } \\
\text { ATE1 }^{-1}\end{array}$ & $\begin{array}{l}\text { Overall chronic rhinosinusitis } \\
\text { symptoms were probably bet- } \\
\text { ter in participants who received } \\
\text { dupilumab. }\end{array}$ \\
\hline Serious adverse events & \multirow{2}{*}{$\begin{array}{l}782 \\
\text { (3 RCTs) }\end{array}$} & \multirow{2}{*}{$\begin{array}{l}\text { RR } 0.45 \\
(0.28 \text { to } \\
0.75)\end{array}$} & Study population & & & \multirow{2}{*}{$\begin{array}{l}\oplus \oplus \odot \odot \\
\text { LOW2 }\end{array}$} & \multirow[b]{2}{*}{$\begin{array}{l}\text { Participants who had dupilumab } \\
\text { may have had fewer serious ad- } \\
\text { verse events than participants who } \\
\text { received placebo in } 3 \text { RCTs ( } 26 / 470 \\
\text { with dupilumab versus } 38 / 312 \text { with } \\
\text { placebo), but we have limited con- } \\
\text { fidence in this estimate because } \\
\text { the sample size may be too small } \\
\text { to estimate this accurately, or cap- } \\
\text { ture the range of adverse events } \\
\text { that could possibly occur in a larg- }\end{array}$} \\
\hline $\begin{array}{l}\text { Follow-up (range): } 16 \text { to } 52 \\
\text { weeks }\end{array}$ & & & $12.2 \%$ & $\begin{array}{l}5.5 \% \\
\text { (3.4 to 9.1) }\end{array}$ & $\begin{array}{l}6.7 \% \text { fewer } \\
\text { ( } 8.8 \text { fewer } \\
\text { to } 3 \text { fewer) }\end{array}$ & & \\
\hline
\end{tabular}




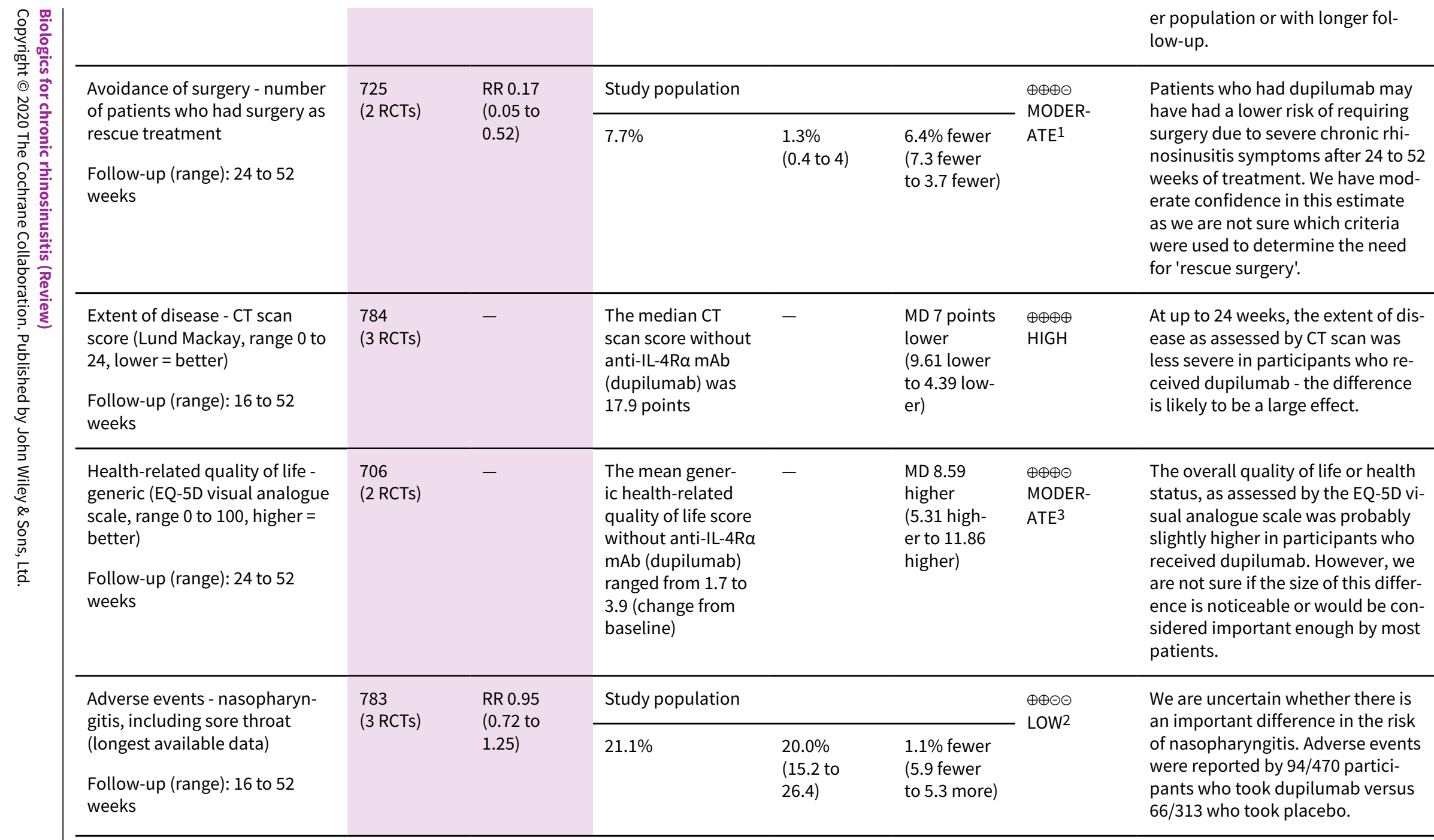

${ }^{*}$ The risk in the intervention group (and its $95 \%$ confidence interval) is based on the assumed risk in the comparison group and the relative effect of the intervention (and its $95 \% \mathrm{Cl})$.

CI: confidence interval; CT: computerised tomography; MD: mean difference; RCT: randomised controlled trial; RR: risk ratio; SNOT-22: Sino-Nasal Outcome Test-22; VAS: visual analogue scale

\section{GRADE Working Group grades of evidence}

High certainty: We are very confident that the true effect lies close to that of the estimate of the effect 
Moderate certainty: We are moderately confident in the effect estimate: The true effect is likely to be close to the estimate of the effect, but there is a possibility that it is substantially different

Low certainty: Our confidence in the effect estimate is limited: The true effect may be substantially different from the estimate of the effect

Very low certainty: We have very little confidence in the effect estimate: The true effect is likely to be substantially different from the estimate of effect

1Downgraded by one level due to study limitations: methods or criteria used in the measurement of the outcome were not validated.

2Downgraded by two levels due to imprecision and indirectness: small sample size for the outcome estimated resulting in an imprecise estimation of effect size. Moreover, some serious adverse events are relatively rare; a larger and more heterogenous population or longer periods of treatment and follow-up may be needed.

3Downgraded by one level due to serious limitations: the criteria used for requiring/not requiring 'rescue surgery' were unclear.

\section{Summary of findings 2. Anti-IL-5 mAb (mepolizumab) compared to placebo (on top of topical steroids) for chronic rhinosinusitis}

\section{Anti-IL-5 mAb (mepolizumab) compared to placebo (on top of topical steroids) for chronic rhinosinusitis}

Patients or population: patients with severe chronic rhinosinusitis with nasal polyps

Setting: tertiary care

Intervention: anti-IL-5 mAb (mepolizumab)

Comparison: placebo (on top of topical steroids)

\begin{tabular}{|c|c|c|c|c|c|c|c|}
\hline \multirow[t]{2}{*}{ Outcomes } & \multirow{2}{*}{$\begin{array}{l}\text { Number } \\
\text { of par- } \\
\text { ticipants } \\
\text { (studies) }\end{array}$} & \multirow{2}{*}{$\begin{array}{l}\text { Relative ef- } \\
\text { fect } \\
(95 \% \mathrm{Cl})\end{array}$} & \multicolumn{3}{|c|}{ Anticipated absolute effects ${ }^{\star}(95 \% \mathrm{Cl})$} & \multirow{2}{*}{$\begin{array}{l}\text { Certainty } \\
\text { of the evi- } \\
\text { dence } \\
\text { (GRADE) }\end{array}$} & \multirow[t]{2}{*}{ What happens } \\
\hline & & & $\begin{array}{l}\text { Without anti-IL-5 mAb } \\
\text { (mepolizumab) }\end{array}$ & $\begin{array}{l}\text { With an- } \\
\text { ti-IL-5 mAb } \\
\text { (mepolizum- } \\
\text { ab) }\end{array}$ & Difference & & \\
\hline $\begin{array}{l}\text { Health-related quality of life - dis- } \\
\text { ease-specific (SNOT-22, range } 1 \text { to } 100 \text {, } \\
\text { lower = better) } \\
\text { Follow-up: } 25 \text { weeks }\end{array}$ & $\begin{array}{l}105 \\
(1 \mathrm{RCT})\end{array}$ & - & $\begin{array}{l}\text { The mean disease-spe- } \\
\text { cific health-related } \\
\text { quality of life score } \\
\text { without anti-IL-5 mAb } \\
\text { (mepolizumab) was } \\
40.36 \text {. }\end{array}$ & - & $\begin{array}{l}\text { MD } 13.26 \text { lower } \\
\text { (22.08 lower to } \\
4.44 \text { lower) }\end{array}$ & $\begin{array}{l}\oplus \oplus \ominus \ominus \\
\text { LOW1 }\end{array}$ & $\begin{array}{l}\text { Aspects of health- } \\
\text { related quality } \\
\text { of life that are di- } \\
\text { rectly impacted } \\
\text { by chronic rhi- } \\
\text { nosinusitis may } \\
\text { have been bet- } \\
\text { ter in participants } \\
\text { who received } \\
\text { mepolizumab but } \\
\text { we are uncertain } \\
\text { about this esti- } \\
\text { mate. }\end{array}$ \\
\hline $\begin{array}{l}\text { Disease severity - VAS (range } 0 \text { to } 10 \text {, } \\
\text { lower = better) } \\
\text { Follow-up: } 25 \text { weeks }\end{array}$ & $\begin{array}{l}72 \\
(1 \mathrm{RCT})\end{array}$ & - & $\begin{array}{l}\text { The mean disease sever- } \\
\text { ity score without an- } \\
\text { ti-IL-5 mAb (mepolizum- } \\
\text { ab) was } 6.21 \text {. }\end{array}$ & - & $\begin{array}{l}\text { MD } 2.03 \text { lower } \\
\text { (3.65 lower to } \\
0.41 \text { lower) }\end{array}$ & $\begin{array}{l}\oplus \ominus \ominus \ominus \\
\text { VERY } \\
\text { LOW } 1,2\end{array}$ & $\begin{array}{l}\text { We are very un- } \\
\text { certain about } \\
\text { the impact of } \\
\text { mepolizumab }\end{array}$ \\
\hline
\end{tabular}




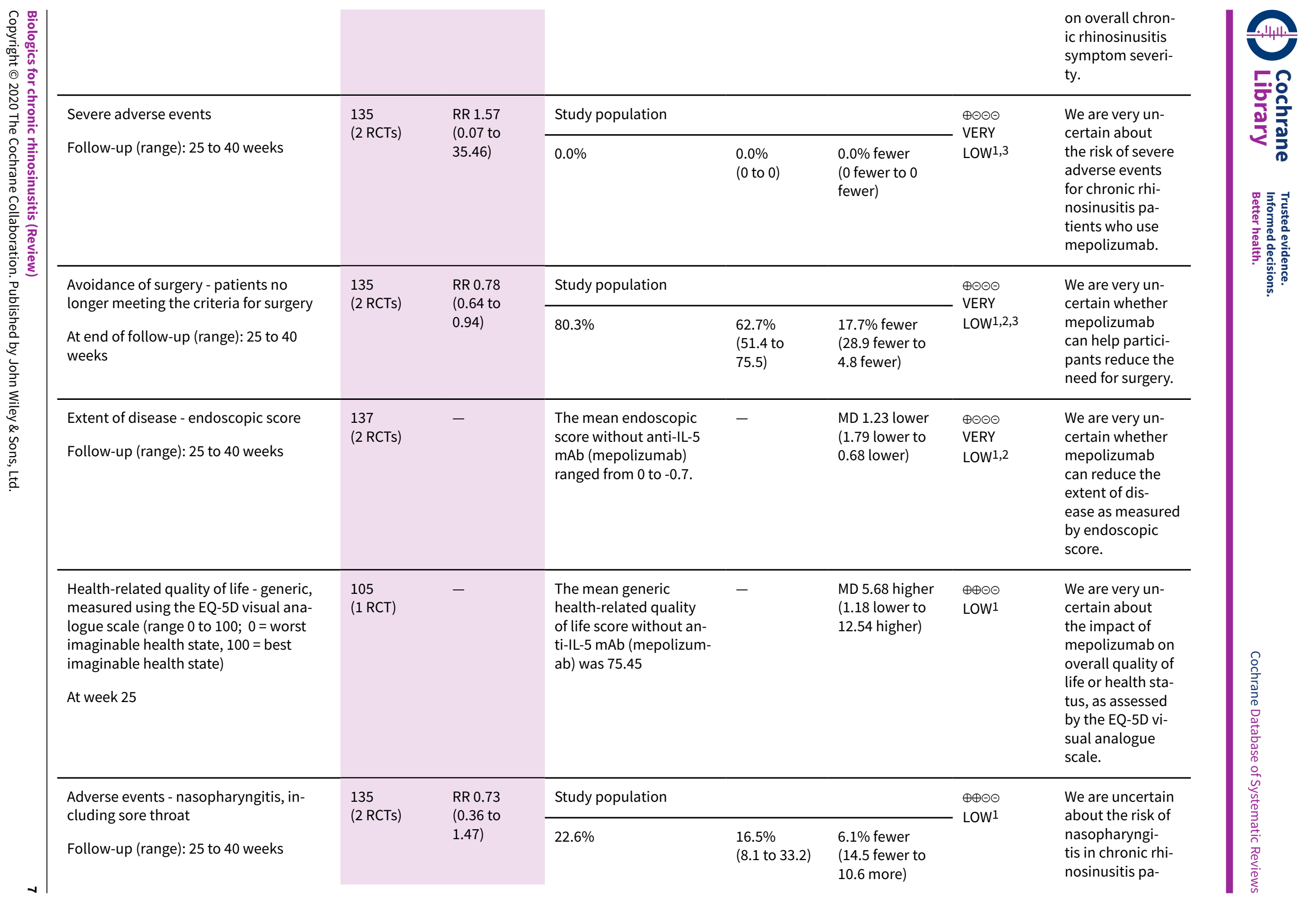


${ }^{*}$ The risk in the intervention group (and its 95\% confidence interval) is based on the assumed risk in the comparison group and the relative effect of the intervention (and its $95 \% \mathrm{Cl})$.

CI: confidence interval; MD: mean difference; RCT: randomised controlled trial; RR: risk ratio; SNOT-22: Sino-Nasal Outcome Test-22; VAS: visual analogue scale

\section{GRADE Working Group grades of evidence}

High certainty: We are very confident that the true effect lies close to that of the estimate of the effect

Moderate certainty: We are moderately confident in the effect estimate: The true effect is likely to be close to the estimate of the effect, but there is a possibility that it is

substantially different

Low certainty: Our confidence in the effect estimate is limited: The true effect may be substantially different from the estimate of the effect

Very low certainty: We have very little confidence in the effect estimate: The true effect is likely to be substantially different from the estimate of effect

1Downgraded by two levels due to imprecision: very small sample size resulting in a very imprecise estimation of effect sizes.

2Downgraded by one level due to study limitations: methods or criteria used in the measurement of the outcome were not validated.

3Downgraded by one level due to indirectness: one study only assessed patients for two doses (Gevaert 2011). The other study evaluated six doses (24 weeks), but had a more than $30 \%$ dropout rate (Bachert 2017). Therefore, the length of follow-up is inadequate and it is unclear whether this evidence related to safety is generalisable.

\section{Summary of findings 3. Anti-IgE mAb (omalizumab) compared to placebo (on top of topical steroids) for chronic rhinosinusitis}

Anti-IgE mAb (omalizumab) compared to placebo (on top of topical steroids) for chronic rhinosinusitis

Patients or population: patients with chronic rhinosinusitis with nasal polyps

Setting: tertiary care

Intervention: anti-IgE mAb (omalizumab)

Comparison: placebo (on top of topical steroids)

\begin{tabular}{|c|c|c|c|c|c|c|c|}
\hline \multirow[t]{2}{*}{ Outcomes } & \multirow{2}{*}{$\begin{array}{l}\text { Number } \\
\text { of par- } \\
\text { ticipants } \\
\text { (studies) }\end{array}$} & \multirow{2}{*}{$\begin{array}{l}\text { Relative ef- } \\
\text { fect } \\
(95 \% \mathrm{CI})\end{array}$} & \multicolumn{3}{|c|}{ Anticipated absolute effects ${ }^{\star}(95 \% \mathrm{Cl})$} & \multirow{2}{*}{$\begin{array}{l}\text { Certainty } \\
\text { of the evi- } \\
\text { dence } \\
\text { (GRADE) }\end{array}$} & \multirow[t]{2}{*}{ What happens } \\
\hline & & & $\begin{array}{l}\text { Without anti-IgE } \\
\text { mAb (omalizumab) }\end{array}$ & $\begin{array}{l}\text { With anti-IgE } \\
\text { mAb (omal- } \\
\text { izumab) }\end{array}$ & Difference & & \\
\hline $\begin{array}{l}\text { Health-related quali- } \\
\text { ty of life- RSOM-31 and } \\
\text { SNOT-20 }\end{array}$ & $\begin{array}{l}38 \\
(2 \mathrm{RCTS})\end{array}$ & \multicolumn{4}{|c|}{$\begin{array}{l}\text { One study found a significant improvement in sleep }(P=0.03) \text { and general } \\
\text { symptoms }(P=0.01) \text { in the omalizumab group compared to baseline, where- } \\
\text { as in the placebo group no significant changes were seen. }\end{array}$} & $\begin{array}{l}\oplus \ominus \ominus \ominus \\
\text { VERY } \\
\text { LOW1,6 }\end{array}$ & $\begin{array}{l}\text { We are very uncertain about } \\
\text { the impact of omalizumab on } \\
\text { health-related quality of life. }\end{array}$ \\
\hline $\begin{array}{l}\text { Follow-up (range): } 20 \\
\text { weeks to } 6 \text { months }\end{array}$ & & \multicolumn{4}{|c|}{$\begin{array}{l}\text { A second study reported that the median change in SNOT- } 20 \text { score was }-1.05 \\
\text { in the omalizumab group and }-0.20 \text { in the placebo group ( } P<0.78 \text { for the dif- } \\
\text { ference between groups). }\end{array}$} & & \\
\hline
\end{tabular}




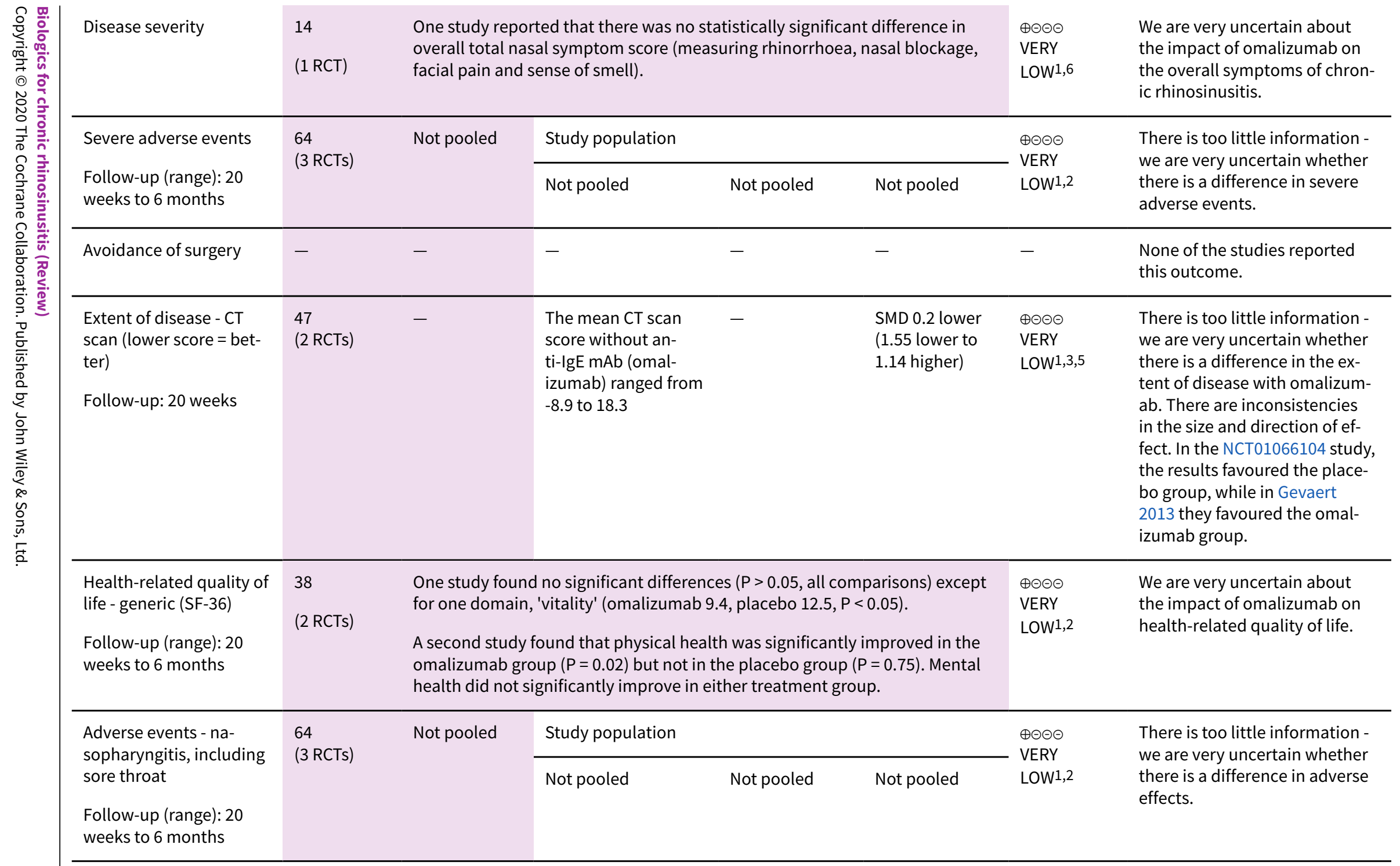

${ }^{*}$ The risk in the intervention group (and its $95 \%$ confidence interval) is based on the assumed risk in the comparison group and the relative effect of the intervention (and its $95 \% \mathrm{Cl})$.

Cl: confidence interval; CT: computerised tomography; RCT: randomised controlled trial; RSOM-31: Rhinosinusitis Outcome Measures-31; SMD: standardised mean difference; SNOT-22: Sino-Nasal Outcome Test-22 
High certainty: We are very confident that the true effect lies close to that of the estimate of the effect

Moderate certainty: We are moderately confident in the effect estimate: The true effect is likely to be close to the estimate of the effect, but there is a possibility that it is substantially different

Low certainty: Our confidence in the effect estimate is limited: The true effect may be substantially different from the estimate of the effect

Very low certainty: We have very little confidence in the effect estimate: The true effect is likely to be substantially different from the estimate of effect

1Downgraded by two levels due to imprecision: very small sample size.

2Downgraded by one level due indirectness: a limited number of doses ( 4 to 12 ) and duration of follow-up (16 to 24 weeks, with most patients followed up for about 4 months). It is unclear how this information on adverse events is generalisable to others.

${ }^{3}$ Downgraded by one level due to study limitations: method of assessment not validated.

${ }^{4}$ Downgraded by one level due to inconsistency: high (unexplained) heterogeneity for the effect sizes.

5Downgraded by one level due to inconsistency: high and unexplained heterogeneity as the size and direction of the effect differed between studies.

${ }^{6}$ Downgraded by one level due to study limitations: rather than overall scores, results were only reported for a very small number of items. 


\section{B A C K G R O U N D}

This review is one of a suite of Cochrane Reviews looking at common management options for patients with chronic rhinosinusitis (Chong 2016a; Chong 2016b; Chong 2016c; Head 2016a; Head 2016b; Head 2016c; Head 2018).

\section{Description of the condition}

Chronic rhinosinusitis represents a common source of ill health; $11 \%$ of UK adults reported chronic rhinosinusitis symptoms in a worldwide population study (Hastan 2011). Symptoms including nasal obstruction, nasal discharge, facial pain, anosmia (loss of sense of smell) and sleep disturbance have a major impact on quality of life, reportedly greater in several domains of the SF-36 than angina or chronic respiratory disease (Gliklich 1995). Acute exacerbations (worsening), inadequate symptom control and respiratory disease exacerbation are common. Complications are rare, but may include visual impairment and intracranial infection.

Two major phenotypes of chronic rhinosinusitis have been described based on the presence or absence of nasal polyps on examination. Nasal polyps are tumour-like hyperplastic swellings of the nasal mucosa, most commonly originating from within the ostiomeatal complex (Larsen 2004). Chronic rhinosinusitis with nasal polyps (CRSwNP) is diagnosed when polyps are seen (on direct or endoscopic examination) in the middle meatus or nasal cavity. Chronic rhinosinusitis without nasal polyps (CRSsNP) is diagnosed when no polyps are observed on examination.

Although the aetiology of chronic rhinosinusitis is not fully understood, it may involve abnormalities in the host response to irritants, commensal and pathogenic organisms and allergens, obstruction of sinus drainage pathways, abnormalities of normal mucociliary function, loss of the normal mucosal barrier or infection. Chronic rhinosinusitis is a heterogenous group of diseases, but three main patterns of inflammation have been identified: type 1 driven, usually associated with chronic rhinosinusitis without nasal polyps; type 2 driven, usually associated with chronic rhinosinusitis with nasal polyps in Caucasian patients; and type 17 driven, associated typically with chronic rhinosinusitis with nasal polyps in Asian patients (Smith 2018). There is some overlap between phenotypes and inflammatory patterns and the current division of chronic rhinosinusitis into two main phenotypes, with and without polyps, is therefore likely to be inadequate for defining patient subgroups. Endotyping, using measurable biomarkers, is increasingly being performed but is not yet routinely incorporated into clinical practice.

Despite the differences in aetiology and phenotype, in clinical practice many treatments for chronic rhinosinusitis are initiated without knowledge of a patient's 'polyp status'. Even when it is known whether or not a patient with chronic rhinosinusitis has polyps, this knowledge does not always suggest adjustments to treatment. This review (and most of its companion reviews) will consider patients with and without polyps together in the initial evaluation of treatment effects. However, as biologics are primarily used in hospital settings and in well-defined patient populations, we planned subgroup analyses to explore potential differences between them (see below).

\section{Description of the intervention}

The term 'biologics' refers to medicinal products produced by a biological process. Monoclonal antibodies are one type of biologic. They target specific inflammatory mediators or immune cells in the pathophysiological pathways that produce chronic inflammatory diseases. Trials have evaluated these agents in conditions such as asthma and atopic dermatitis leading to growing interest in the possibility of using them to treat patients with chronic rhinosinusitis.

\section{How the intervention might work}

Monoclonal antibodies work on different target substances or receptors in the inflammatory pathway. The more we understand about the inflammatory pathways involved in chronic rhinosinusitis, the more we may be able to affect those pathways with biologics. Different biologics are likely to have very different efficacy in different patient populations depending on the pattern of inflammation in those patients. Recent trials in patients with chronic rhinosinusitis with nasal polyps have focused on biologics directed at the inflammatory mediators and receptors involved in type 2 pathways. As yet none have investigated the effectiveness of biologics in type 1 or type 17 driven inflammation.

Currently, biologics are mainly used in patients with severe chronic rhinosinusitis where pharmacological therapy does not provide adequate symptom control, with the aim of reducing those symptoms and leading to an improvement in their quality of life. Some patients with severe chronic rhinosinusitis undergo surgical treatment aimed at achieving these goals. If patients respond well to biologics, surgical intervention may be avoided. If biologics are successful in reducing inflammation and reducing the size of nasal polyps, this should also be visible using endoscopy and computerised tomography (CT) scans. These changes can be documented and quantified using the relevant scoring system.

Biologics are, however, associated with adverse reactions that may be immune-related and can be serious - such as anaphylaxis. Biologics are widely used in rheumatology and some of the serious adverse events documented in those patients include tuberculosis reactivation, lymphoma and severe infections (Singh 2011; Tarp 2017). Another adverse reaction is pharyngitis, which may be serious enough for patients to discontinue treatment.

The following are descriptions of a number of classes and mechanisms of actions of monoclonal antibodies (mAb) with some specific named biologics. This is not an exhaustive list. The field is growing and our understanding of the mechanisms of action may change over time. Biologics not listed here may be evaluated in this review.

\section{Anti-IL-4Ra mAb and anti-IL-13 mAb}

Dupilumab, delivered by subcutaneous injection, is a human monoclonal antibody of the IgG4 subclass that targets the IL-4Ra subunit and disrupts IL-4 and IL-13 signalling. This is involved in the type 2 inflammatory pathway most typically seen in patients with chronic rhinosinusitis with nasal polyps. Trials of dupilumab in asthma have also shown improvement in the symptoms of coexisting chronic rhinosinusitis (Wenzel 2016). Lebrikizumab and tralokinumab are anti-IL-13 monoclonal antibodies. 


\section{Anti-IL-5 mAb}

Mepolizumab, reslizumab and benralizumab are delivered subcutaneously or intravenously, and are human monoclonal $\left(\mathrm{IgG}_{1}\right)$ antibodies targeting interleukin 5 (IL-5) or the IL-5 receptor a subunit on the surface of eosinophil white blood cells. IL-5 promotes eosinophil development survival, so targeting IL-5 reduces blood and tissue eosinophil counts. Mepolizumab is currently approved by the UK's National Institute for Health and Care Excellence (NICE) for the treatment of severe eosinophilic asthma and as IL-5 has been suggested as a parallel marker for the severity of both asthma and chronic rhinosinusitis with nasal polyps, it has the potential to treat both simultaneously (Chupp 2017; Dasgupta 2017; Pavord 2012). Reslizumab and benralizumab have had early success in patients with poorly controlled asthma (DuBuske 2018; Máspero 2017).

\section{Anti-IgE mAb}

Omalizumab, also delivered subcutaneously, is a recombinant DNA-derived humanised $\left(\lg G_{1 k}\right)$ monoclonal antibody that specifically binds to free human immunoglobulin $E$ (IgE) in the blood and interstitial fluid, and to the membrane-bound form of IgE (mIgE) on the surface of mlgE-expressing B-lymphocytes. It therefore has the effect of reducing the levels of IgE in the serum and tissues, with a subsequent blocking of the IgE-mediated inflammatory cascade. This anti-IgE treatment has to date been shown to be effective in allergic rhinitis and asthma (Casale 2001; Hanania 2011).

Further information about the mechanisms of action of biologics in this field can be found in Kariyawasam 2019.

\section{Why it is important to do this review}

To date much of the literature around the role of these new drugs has been focused on the allergy, asthma and immunology subspecialties. As the role for biologic therapies in chronic rhinosinusitis continues to be defined and pharmaceutical companies are now targeting this condition, it is increasingly important for practising otorhinolaryngologists, especially subspecialist rhinologists, to determine the place of biologics in the treatment cascade by keeping up-to-date on their progression. NICE is currently conducting a health technology appraisal of the clinical and cost-effectiveness of dupilumab for chronic rhinosinusitis with nasal polyps (NICE 2019). This Cochrane Review looks at the balance of benefits and harms for biologic drugs in the treatment of patients with chronic rhinosinusitis. It also serves to identify areas for future research, especially as the knowledge of specific chronic rhinosinusitis endotypes increases.

This review is a living systematic review, whereby we will search key databases monthly and update the review as and when new important evidence is found. A living systematic review approach is appropriate for this review because: 1 ) the topic is important for health care decision-making; 2) there is uncertainty about the existing evidence; and 3 ) this is a rapidly developing field where new trials are being actively planned and completed. We will revisit the scope (population, intervention, comparison, outcomes) of the review yearly, or more frequently as appropriate, to ensure that new agents or uses are included as this field develops. In addition to having more data on safety and efficacy, our understanding of how biologics work, the best way to measure outcomes and how outcomes are interpreted will very likely change as more research is completed. Therefore, we will adapt our definition of what outcomes to measure and how outcomes should be measured and interpreted over time.

\section{O B JECTIVES}

\section{Main objective}

To assess the effects of biologics for the treatment of chronic rhinosinusitis.

\section{Secondary objective}

To maintain the currency of the evidence, using a living systematic review approach.

\section{METHODS}

\section{Criteria for considering studies for this review Types of studies}

Randomised controlled trials and quasi-randomised trials.

We only considered cross-over trials if there was sufficient evidence to suggest that the condition of patients was stable and the washout period was adequate. Otherwise, we only planned to use the first phase of cross-over trials.

We only included studies where patients were followed up for at least three months, to reflect the importance of focusing on longterm outcomes for a chronic condition.

\section{Types of participants}

Patients with chronic rhinosinusitis, whether with polyps (CRSwNP) or without polyps (CRSsNP).

We excluded studies that had included a majority of patients with:

- cystic fibrosis;

- allergic fungal sinusitis/eosinophilic fungal/mucinous rhinosinusitis;

- antrochoanal polyps (benign polyps originating from the mucosa of the maxillary sinus);

- malignant polyps;

- primary ciliary dyskinesia;

- a history of surgery for nasal polyps within three months of entry to the study.

\section{Types of interventions}

\section{Intervention}

All monoclonal antibodies used for the treatment of chronic rhinosinusitis. This included but was not limited to the following:

- anti-IL-4Ra mAb (dupilumab);

- anti-IL-13 (lebrikizumab, tralokinumab);

- anti-IL-5 mAb (reslizumab, benralizumab, mepolizumab);

- anti-IgE mAb (omalizumab).

These are the biologics identified in November 2019 as most likely to be used in patients with chronic rhinosinusitis. Additional monoclonal antibodies and other classes of biologics will also be 
included in this review when they are evaluated in patients with chronic rhinosinusitis.

All routes of administration, doses and duration of treatment were included. However, studies should have followed up participants for three months or more.

\section{Comparison}

Placebo or no treatment. Surgery will be an alternative treatment (comparison) when trials in the area become available.

\section{Concurrent treatments}

It was expected that most studies would have used intranasal steroids as a concurrent treatment. There was no limitation on the type of pharmacological concurrent treatments used.

\section{Comparison pairs}

The following main comparison pairs were proposed in the protocol (Chong 2019):

- anti-IL-4Ra mAb plus intranasal steroids versus placebo/no treatment plus intranasal steroids;

- anti-IL-13 plus intranasal steroids versus placebo/no treatment plus intranasal steroids;

- anti-IL-5 mAb plus intranasal steroids versus placebo/no treatment plus intranasal steroids;

- anti-IgE mAb plus intranasal steroids versus placebo/no treatment plus intranasal steroids.

\section{Types of outcome measures}

We analysed the following outcomes in the review, but we did not use them as a basis for including or excluding studies.

Our primary intention was to assess the effects of assignment, rather than adherence to treatment.

\section{Primary outcomes}

- Health-related quality of life, using validated disease-specific health-related quality of life scores, such as the SinoNasal Outcome Test-22 (SNOT-22), Rhinosinusitis Outcome Measures-31 (RSOM-31) and SNOT-20.

- Disease severity, as measured by validated patient-reported symptom score (such as the Chronic Sinusitis Survey (CSS) questionnaire and visual analogue scales). Where this was unavailable, we considered including data measuring the severity of individual symptoms (see below).

- Serious adverse events (SAEs), measured by the number of participants affected. A serious adverse event is defined as "Death, a life-threatening adverse event, inpatient hospitalisation or prolongation of existing hospitalisation, a persistent or significant incapacity or substantial disruption of the ability to conduct normal life functions, or a congenital anomaly/birth defect. Important medical events that may not result in death, be life-threatening, or require hospitalisation may be considered serious when, based upon appropriate medical judgment, they may jeopardize the patient or subject and may require medical or surgical intervention to prevent one of the outcomes listed in this definition" (FDA 2018).
Many studies within this suite of reviews (Chong 2016a; Chong 2016b; Chong 2016c; Head 2016a; Head 2016b; Head 2016c; Head 2018) did not use/present data using instruments that were either validated or evaluated all four types of symptoms meeting the EPOS 2012 diagnostic criteria in a composite score. If data from a validated score were unavailable, we planned to analyse data related to each of these individual symptoms, if presented.

\section{Secondary outcomes}

- Avoidance of surgery, measured by the number (proportion) of participants who had, or did not have, surgery for chronic rhinosinusitis symptoms, or who no longer fulfilled the eligibility criteria for surgery ${ }^{\star}$. (See comments in Assessment of risk of bias in included studies).

- Extent of disease as measured by either:

* endoscopic score (depending on population, either nasal polyps size score or other such as Lund Kennedy); and/or

* computerised tomography (CT) scan score (e.g. Lund Mackay with a range of 0 to 24 , higher $=$ worse) .

- Health-related quality of life, using generic quality of life scores, such as the SF-36, EQ-5D and other well-validated instruments.

- Adverse effects: nasopharyngitis, including sore throat.

Outcomes were measured at 3 to 6 months, 6 to 12 months and more than 12 months. For adverse events, we analysed data from the longest time periods.

*We recorded and tabulated the eligibility criteria for surgery used in the included studies.

\section{Search methods for identification of studies}

The Cochrane ENT Information Specialist conducted systematic searches for randomised controlled trials and controlled clinical trials. There were no language, publication year or publication status restrictions. The date of the search was 16 September 2019.

\section{Electronic searches}

The Information Specialist searched:

- the Cochrane ENT Register (searched via the Cochrane Register of Studies 18 September 2019);

- the Cochrane Central Register of Controlled Trials (CENTRAL 2019, Issue 9) (searched via the Cochrane Register of Studies);

- Ovid MEDLINE(R) Epub Ahead of Print, In-Process \& Other NonIndexed Citations, Ovid MEDLINE(R) Daily and Ovid MEDLINE(R) (1946 to 16 September 2019);

- Ovid EMBASE (1974 to 16 September 2019);

- Web of Science (1945 to 16 September 2019);

- ClinicalTrials.gov, www.clinicaltrials.gov (searched via the Cochrane Register of Studies to 18 September 2019);

- ClinicalTrials.gov, www.clinicaltrials.gov (searched via www.clinicaltrials.gov to date 18 September 2019);

- World Health Organization (WHO) International Clinical Trials Registry Platform (ICTRP) (searched via the Cochrane Register of Studies to 18 September 2019);

- World Health Organization (WHO) International Clinical Trials Registry Platform (ICTRP) (searched 18 September 2019). 
The Information Specialist modelled subject strategies for databases on the search strategy designed for CENTRAL, Ovid MEDLINE and Ovid Embase. Where appropriate, they were combined with subject strategy adaptations of the highly sensitive search strategy designed by Cochrane for identifying randomised controlled trials and controlled clinical trials (as described in the Cochrane Handbook for Systematic Reviews of Interventions Version 5.1.0, Box 6.4.b. (Handbook 2011). Search strategies for major databases including CENTRAL are provided in Appendix 1.

\section{Living systematic review considerations}

As a living systematic review, the Information Specialist will conduct monthly searches of:

- the Cochrane ENT Trials Register (search via the Cochrane Register of Studies to date);

- the Cochrane Central Register of Controlled Trials (CENTRAL) (search via the Cochrane Register of Studies to date);

- Ovid MEDLINE(R) Epub Ahead of Print, In-Process \& Other NonIndexed Citations, Ovid MEDLINE(R) Daily and Ovid MEDLINE(R) (1946 to date):

- Ovid Embase (1974 to date);

- Web of Knowledge, Web of Science (1945 to date);

- ClinicalTrials.gov, www.clinicaltrials.gov (search via the Cochrane Register of Studies to date);

- World Health Organization (WHO) International Clinical Trials Registry Platform (ICTRP) (search via the Cochrane Register of Studies to date).

To facilitate these searches the Information Specialist will set up monthly auto-alerts where available and appropriate.

The Information Specialist will also conduct quarterly searches of the following sources, and prior to publication of any update:

- ClinicalTrials.gov (search via www.clinicaltrials.gov to date);

- World Health Organization (WHO) International Clinical Trials Registry Platform (ICTRP) (search to date).

A summary of trials identified versus reports obtained will be published in the review.

Biologics are a new class of intervention. The search strategy developed is highly sensitive, in order to try to capture new interventions as they are introduced. The Information Specialist will review the search methods (the sources and search frequency) and the search terms (index terms and free text terms) on an annual basis. The aim will be to include new terms for new interventions as they are introduced, and remove terms to increase precision as interventions are removed or withdrawn.

\section{Searching other resources}

We scanned the reference lists of identified publications for additional trials and contacted trial authors where necessary. In addition, the Information Specialist searched Ovid MEDLINE to retrieve existing systematic reviews relevant to this systematic review, so that we could scan their reference lists for additional trials.

We did not perform a separate search for adverse effects. We considered adverse effects described in included studies only.

\section{Clinical study reports (CSRs) and other sources of evidence}

This review meets many of the 18 criteria for considering clinical study reports as a source of evidence (Jefferson 2018). In particular, there is a concern about publication bias with a new class of drugs for this current condition. Moreover, these are very costly agents that are already marketed for other conditions and there is a risk of off-label use.

There are no established search procedures to identify clinical study reports at the time of publication. We attempted to identify unpublished studies and clinical study reports. The Information Specialist searched:

1. Regulatory bodies: We searched the websites of the:

a. US Food and Drug Administration (FDA) (http://www.fda.gov and https://www.fda.gov/about-fda/about-website/fdagovarchive) (searched 11 December 2019);

b. European Medicines Agency (EMEA) (http:// www.emea.europa.eu) (searched 18 November 2019);

c. European Union Clinical Trials Register (EUCRT) (https:// www.clinicaltrialsregister.eu/) (searched 15 November 2019).

2. Manufacturer-specific clinical trial repositories and data sharing platforms:

- Novartis Clinical Trial Results Database (https:// www.novctrd.com) (searched 18 November 2019);

- GSK Study Register (https://www.gsk-studyregister.com) (searched 18 November 2019).

3. Direct requests to manufacturers: We did not identify additional trials and therefore did not write to the manufacturer/ sponsors. We plan to contact the principal investigators/ manufacturers/sponsors of each of the known trials individually to ask for additional data as part of the planned update of this living systematic review. We did identify one clinical study report (Bachert 2017) and additional data from ClinicalTrials.gov and EUCTR for five included studies (Bachert 2016; Bachert 2017; LIBERTY SINUS 24; LIBERTY SINUS 52; NCT01066104), which were identified as part of the regular electronic searches.

\section{Living systematic review considerations}

As a living systematic review, the Information Specialist will conduct quarterly searches to retrieve existing systematic reviews relevant to this systematic review and monthly searches of the Web of Knowledge Science Citation Index for articles referencing the published review and its included studies. Google Scholar searches will be conducted on an annual basis. We will review on an ongoing basis (and at least every six months) the various sources to search for clinical study reports, updating the list of sources searched and when as required. We will make contact with the principal investigators of ongoing trials and ask them to advise when results are available, or to share early or unpublished data.

We have a number of plans to investigate further the identification of clinical study reports and other sources of evidence. These are detailed in Differences between protocol and review.

\section{Data collection and analysis}

\section{Selection of studies}

The Cochrane ENT Information Specialist used Cochrane's Screen4Me workflow to help assess the initial search results for the first iteration of this living systematic review because of the high 
number of results retrieved from the database searches. Screen $4 \mathrm{Me}$ comprises three components: 1) known assessments - a service that matches records in the search results to records that have already been screened in Cochrane Crowd and been labelled as 'a RCT' or as 'not a RCT'. 2) The machine learning classifier (RCT model) (Wallace 2017), available in the Cochrane Register of Studies (CRS-Web), assigns a probability of being a true RCT (from 0 to $100)$ to each citation. For citations that are assigned a probability score below the cut-point at a recall of $99 \%$ we have assumed these to be non-RCTs. For those that score on or above the cut-point we either manually dual screened these results or sent them to 3 ) Cochrane Crowd for screening (Cochrane's citizen science platform where the Crowd help to identify and describe health evidence). For more information about Screen4Me and the evaluations that have been done, please go to the Screen4Me website on the Cochrane Information Specialist's portal and see Marshal 2018, McDonald 2017, Noel-Storr 2018 and Thomas 2017.

At least two review authors (LYC/PP), or the Cochrane ENT Information Specialist (SC) acting as one screener, independently screened the remaining titles and abstracts to identify potentially relevant studies. At least two review authors (MB/PP/SS) independently evaluated the full text of each potentially relevant study to determine whether it met the inclusion/exclusion criteria for this review.

We resolved any differences by discussion and consensus, with the involvement of a third author (KS) for clinical and/methodological input where necessary.

\section{Living systematic review considerations}

We will immediately collate and screen any new citations retrieved by the monthly searches using the approach outlined above including, as a first step in monthly screening, applying the Screen4Me workflow starting with the RCT model.

\section{Data extraction and management}

One review author (MB) and one of two Cochrane ENT methodologists (AT/KW, listed in the Acknowledgements) independently extracted outcome data from each study using a standardised data collection form (see Appendix 2). Whenever a study had more than one publication, we retrieved all publications to ensure complete extraction of data. Where there were discrepancies in the data extracted by different review authors, we checked these against the original reports and resolved differences by discussion and consensus, with the involvement of a third author (MB) or a methodologist (LYC) where appropriate. We contacted the original study authors for clarification or for missing data whenever possible. If differences were found between publications of a study, we contacted the original authors for clarification. We used data from the main paper(s) if no further information was found.

In addition, we also compared trials identified through study registers with identified publications. If an unpublished trial was identified (registered in trial registry, but more than 12 months since completion of recruitment and no data/incomplete data published), we contacted the contact person listed in the trial registry websites for information. Whenever clinical study reports or data from regulatory bodies are available, we will compare these against the journal reports and use them as the primary source of data if there is a discrepancy in the information. However, current experience with the use of clinical study reports suggests that there is often a considerable time lag between requesting these data and obtaining them. Therefore, we will make use of data from journal reports as the main source of evidence as a starting point and then check the data against the clinical study reports and regulatory data as and when these are available.

We included key characteristics of the studies, such as study design, setting, sample size, population and how outcomes were defined or collected in the studies. In addition, we also collected baseline information on prognostic factors or effect modifiers. For this review, this included:

- presence or absence of nasal polyps;

- polyp score (where applicable);

- whether the patient has had previous sinus surgery.

The primary effect of interest is the effect of treatment assignment, which reflects the outcomes of treatment for people who were prescribed the intervention rather than per protocol analysis (the effect on people who completed the full course of treatment as planned). For the outcomes of interest to the review, we extracted the findings from the studies on an available case analysis basis, i.e. we included available data from all participants at the time points based on the treatment randomised whenever possible, irrespective of compliance or whether patients had received the treatment as planned.

In addition to extracting pre-specified information about study characteristics and aspects of methodology relevant to risk of bias, we extracted the following summary statistics for each trial and each outcome:

- For continuous data: the mean values, standard deviations and number of patients for each treatment group. Where endpoint data were not available, we extracted the values for change from baseline. We analysed data from measurement scales such as SNOT-22 and EQ-5D as continuous data.

- For binary data: the number of participants experiencing an event and the number of patients assessed at the time point.

- For ordinal scale data: if the data appeared to be approximately normally distributed or if the analysis that the investigators performed suggested parametric tests were appropriate, then we treated the outcome measures as continuous data. Alternatively, if data were available, we planned to convert into binary data.

We prespecified the time points of interest for the outcomes in this review. While studies may report data at multiple time points, we only extracted the longest available data within the time points of interest. For example, for 'short' follow-up periods, our time point was defined as three to six months post-randomisation. If a study reported data at three, four and six months, we only extracted and analysed the data for the six-month follow-up.

\section{Assessment of risk of bias in included studies}

Two Cochrane ENT methodologists (AT/KW, listed in the Acknowledgements) independently assessed the risk of bias of each included study.

In the first version of the review, we used the original version of the Cochrane 'Risk of bias' tool (ROB-1) (Handbook 2011). For future 
versions of this living systematic review, we anticipate using the Cochrane 'Risk of bias 2.0' tool (ROB-2) (Sterne 2019), according to the guidance in the latest version of the Cochrane Handbook for Systematic Reviews of Interventions (version 6; Handbook 2019).

When using the ROB-1 tool, we followed the guidance in the Cochrane Handbook for Systematic Reviews of Interventions (version 5; Handbook 2011). We assessed the risk of bias as 'low', 'high' or 'unclear' for each of the following six domains:

- sequence generation;

- allocation concealment;

- blinding of participants, personnel and outcome assessment;

- incomplete outcome data;

- selective reporting;

- other sources of bias (if required).

In future iterations of this living systematic review, we plan to apply the ROB-2 tool (rather than ROB-1) according to the guidance in the latest version of the Cochrane Handbook for Systematic Reviews of Interventions (Handbook 2019). We will assess the risk of bias as 'low', 'high' or 'some concerns' for each of the following five domains:

- risk of bias arising from the randomisation process;

- risk of bias due to deviations from the intended interventions;

- risk of bias due to missing outcome data;

- risk of bias in measurement of outcome;

- risk of bias in selection of the reported result.

For ROB-2, we will only assess the outcomes included in the 'Summary of findings' table.

For the outcome 'disease severity, as measured by validated patient-reported symptom score' we will only conduct a ROB-2 assessment if this is reported. If only the results from individual symptoms, or non-validated scores, are reported we will not individually assess these, as the risk of bias is likely to be present due to the choice of outcome measure and selective reporting of only certain aspects of the condition.

There is a particular risk of bias in assessing the outcome 'avoidance of surgery', as there are no widely accepted criteria to determine when patients should or should not have surgery. Unless studies explicitly specify what criteria are used for making judgements and both the investigator (offering/deciding on the surgery) and participants were blinded, there are potential biases in the decision-making process of the study personnel in determining whether or not a participant fulfils the criteria for surgery and/ or whether they should be offered the option of surgery. We assessed this in the 'Blinding, outcomes assessment' domain using the ROB-1 tool and we will assess this in the 'Risk of bias in the measurement of outcome' domain when we are using the ROB-2 tool.

\section{Measures of treatment effect}

We summarised the effects of dichotomous outcomes (e.g. proportion of patients with symptom resolution) as risk ratios (RR) with 95\% confidence intervals (Cls). For the key outcomes that we presented in the 'Summary of findings' tables, we also expressed the results as absolute numbers based on the pooled results and compared to the assumed risk. If appropriate, we would also have considered calculating the number needed to treat to benefit (NNTB) using the pooled results. The assumed baseline risk is typically either (a) the median of the risks of the control groups in the included studies, this being used to represent a 'medium-risk population' or, alternatively, (b) the average risk of the control groups in the included studies is used as the 'study population' (Handbook 2019). If a large number of studies are available, and where appropriate, we may also present additional data based on the assumed baseline risk in (c) a low-risk population and (d) a high-risk population.

For continuous outcomes, we expressed treatment effects as a mean difference (MD) with standard deviation (SD) or as a standardised mean difference (SMD) if different scales had been used to measure the same outcome. We provided a clinical interpretation of the SMD values using either Cohen's $d$ or by conversion to a recognised scale if possible.

\section{Unit of analysis issues}

Cross-over trials and cluster-randomised trials are unlikely for this review topic. We did not plan to use data from phase II of crossover studies (unless there was sufficient evidence to suggest that the condition of patients was stable and the washout period was adequate). If these trial designs are found and deemed suitable to use in the future, we will seek advice from the Cochrane Bias Methods Group and use the latest version of the ROB-2 tool for cross-over and cluster-randomised trials.

We expected that studies would take multiple measurements or observations of a single outcome in the same patients (repeated measurements). In these situations, we only extracted and analysed the data point for the longest available follow-up specified in our protocol (Chong 2019).

\section{Dealing with missing data}

We tried to contact study authors via email whenever the outcome of interest was not reported, if the methods of the study suggest that the outcome had been measured. We did the same if not all data required for meta-analysis had been reported, unless the missing data were standard deviations. If standard deviation data were not available, we approximated these using the standard estimation methods from $P$ values, standard errors or $95 \%$ Cls where reported, as detailed in the Cochrane Handbook for Systematic Reviews of Interventions (Handbook 2019). If it was impossible to estimate these, we planned to contact the study authors.

Apart from imputations for missing standard deviations, we conducted no other imputations. We will extracted and analysed all data using the available case analysis method.

\section{Assessment of heterogeneity}

We assessed clinical heterogeneity (which may be present even in the absence of statistical heterogeneity) by examining the included trials for potential differences between studies in the types of participants recruited, interventions or controls used and the outcomes measured.

We assessed statistical heterogeneity by visually inspecting the forest plots and by considering the $\mathrm{Chi}^{2}$ test (with a significance level set at $P$ value $<0.10$ ) and the $I^{2}$ statistic, which calculates the 
percentage of variability that is due to heterogeneity rather than chance (Handbook 2019).

\section{Assessment of reporting biases}

We assessed reporting bias as between-study publication bias and within-study outcome reporting bias.

\section{Outcome reporting bias (within-study reporting bias)}

We assessed within-study reporting bias by comparing the outcomes reported in the published report against the study protocol, whenever this could be obtained. If the protocol or trial registry entry was not available, we compared the outcomes reported to those listed in the methods section. If results are mentioned but not reported adequately in a way that allows analysis (e.g. the report only mentions whether the results were statistically significant or not), bias in a meta-analysis is likely to occur. We sought further information from the study authors. If no further information could be found, we planned to note this as being a 'high' risk of bias when the ROB-1 tool was used. If there was insufficient information to judge the risk of bias we noted this as an 'unclear' risk of bias (Handbook 2011). When the ROB-2 tool is used in the future, we will assess selective reporting bias in a similar way, according to the signalling questions in the 'risk of bias in selection of the reported result' domain (Handbook 2019). However, we will assess selective non-reporting bias at the synthesis level, using the latest tools (e.g. ROB-ME) if available.

\section{Publication bias (between-study reporting bias)}

We planned to assess funnel plots if sufficient studies (more than 10) were available for an outcome. If we had observed asymmetry of the funnel plot, we would have conducted more formal investigation using the methods proposed by Egger 1997. We also report on whether there were any studies identified through trial registries and other sources (Searching other resources), with unpublished reports.

\section{Data synthesis}

We conducted all meta-analyses using RevMan Web (RevMan Web 2019). For dichotomous data, we planned to analyse treatment differences as a risk ratio (RR) calculated using the Mantel-Haenszel methods.

For continuous outcomes, if all the data were from the same scale, we pooled mean values obtained at follow-up with change outcomes and reported this as a MD. However, if the SMD had to be used as an effect measure, we did not pool change and endpoint data.

We proposed using a random-effects model since it was likely that there would be clinical heterogeneity in the response to different types of biologics or different types of monoclonal antibodies. However, we also planned to undertake a sensitivity analysis to examine the effects of using the alternative fixed-effect model.

\section{Living systematic review considerations}

\section{When new evidence will be incorporated into the living systematic review}

Whenever new evidence (meaning studies, data or information) relevant to the review is identified, we will extract the data and assess risk of bias, as appropriate. We will immediately incorporate any important new evidence into the review.

We will not adjust the meta-analyses to account for multiple testing, given that the methods related to frequent updating of metaanalyses are under development (Simmonds 2017). We will not use sequential methods for updated meta-analyses (Handbook 2019).

\section{Subgroup analysis and investigation of heterogeneity}

When studies had a mixed group of patients, we planned to analyse the study as one subgroup (rather than as a mixed group) if more than $80 \%$ of patients belonged to one category. For example, if $81 \%$ of patients had chronic rhinosinusitis without nasal polyps, we would analyse the study as that subgroup.

We planned to conduct subgroup analyses based on the phenotypes of patients (whether patients had chronic rhinosinusitis with or without nasal polyps, are a mixed group or the status of polyps is not known or not reported) regardless of whether statistical heterogeneity was observed, as these are widely suspected to be potential effect modifiers. Although there appears to be a considerable overlap between the two forms of chronic rhinosinusitis with regards to inflammatory profile, clinical presentation and effect of treatment (Cho 2012; DeMarcantonio 2011; Ebbens 2010; EPOS 2007; Ragab 2004; Ragab 2010; van Drunen 2009), there is some evidence pointing to differences in the respective inflammatory profiles (Kern 2008; Keswani 2012; Tan 2011; Tomassen 2011; Zhang 2008; Zhang 2009), and potentially even differences in treatment outcome (Ebbens 2011).

We planned to present this as the main subgroup analysis for effectiveness outcomes in this review. We planned to present all other subgroup analysis results in tables.

In addition to subgrouping by phenotype, we planned to conduct the following subgroup analyses in the presence of statistical heterogeneity:

- Patients with asthma as a comorbidity. Patients with asthma may have different inflammatory markers and respond differently. In addition to chronic rhinosinusitis symptoms, they may also benefit from better control of asthma symptoms. However, there are no clear data to tell us which patients will benefit more or less from certain types of biologics, therefore the direction of effects is unclear.

- Patients with non-steroidal anti-inflammatory drug (NSAID)exacerbated respiratory disease ( $\mathrm{N}$-ERD). The rationale is similar to that for patients with asthma as a comorbidity.

- Treatment regimens. For agents acting on the same target substance or receptor, treatment regimens such as dose and frequency of initial treatment and maintenance treatment are likely to be important. However, at the preparation of the protocol in 2019 there was not enough information to inform how these subgroups should be defined. We will revisit this question as part of our regular re-evaluation of the review methods, as and when more data are available from trials.

\section{Sensitivity analysis}

We planned to carry out sensitivity analyses to determine whether the findings are robust to the decisions made in the course of identifying, screening and analysing the trials. We planned to 
conduct sensitivity analysis for the following factors, if there were relevant data to do so:

- risk of bias of included studies: excluding studies with high risk of overall bias for the results, as assessed using the Cochrane ROB-1 and ROB-2 tools;

- impact of model chosen: fixed-effect versus random-effects model;

- how outcomes were measured: we planned to investigate the impact of including data where the validity of the measurement was unclear.

If any of these investigations found a difference in the size of the effect or heterogeneity, we would mention this in the 'Effects of interventions' section. However, there were insufficient studies and data meeting these criteria and these analysis were not required.

\section{Summary of findings and assessment of the certainty of the evidence}

We used the GRADE approach to rate the overall certainty of evidence for each outcome using the GDT tool (https:// gradepro.org/) for the main comparison pairs listed in the Types of interventions section. The certainty of evidence reflects the extent to which we are confident that an estimate of effect is correct and we applied this in the interpretation of results. There are four possible ratings: 'high', 'moderate', 'low' and 'very low'. A rating of 'high' certainty evidence implies that we are confident in our estimate of effect and that further research is very unlikely to change our confidence in the estimate of effect. A rating of 'very low' certainty implies that any estimate of effect obtained is very uncertain.

The GRADE approach rates evidence from RCTs that do not have serious limitations as high certainty. However, several factors can lead to the downgrading of the evidence to moderate, low or very low. The degree of downgrading is determined by the seriousness of these factors:

- study limitations (risk of bias);

- inconsistency;

- indirectness of evidence;

- imprecision;

- publication bias.
The 'Summary of findings' tables present only the seven top priority outcomes (primary outcomes: disease-specific healthrelated quality of life, disease severity as measured by validated patient-reported symptom score, serious adverse events (SAEs) and secondary outcomes: avoidance of surgery, extent of disease as measured by endoscopic score or CT scan score, generic healthrelated quality of life and other adverse effects).

\section{Methods for future updates}

We will review the scope and methods of this review approximately yearly (or more frequently if appropriate) in the light of potential changes in the topic area, or the evidence being included in the review (for example, additional comparisons, interventions or outcomes, or new review methods available).

\section{Conditions under which the review will no longer be maintained as a living systematic review}

The review will no longer be maintained as a living systematic review once there is high-certainty evidence obtained for the primary effectiveness outcomes of the review; new studies are not expected to be conducted regularly for the interventions included in this review; or the review topic is no longer a priority for health care decision-making.

\section{RES U L T S}

\section{Description of studies}

\section{Results of the search}

The searches retrieved a total of 4914 references. This reduced to 3341 after the removal of duplicates. The Cochrane ENT Information Specialist sent all 3341 references to the Screen4Me workflow. The Screen4Me workflow identified 399 references as having been previously assessed: 179 had been rejected as not RCTs and 220 had been assessed as possible RCTs. The RCT classifier rejected an additional 1253 references as not RCTs (with a $99 \%$ sensitivity). The Cochrane Crowd assessed the remaining 1689 references, rejecting 1046 as not RCTs and identifying 643 as possible RCTs. Following this process, the Screen4Me workflow had therefore identified 863 possible RCTs for title and abstract screening.

The results of this process are detailed in the PRISMA flow diagram (Figure 1) and summarised in the table below. 
Figure 1. PRISMA flow diagram for initial search (September 2019).

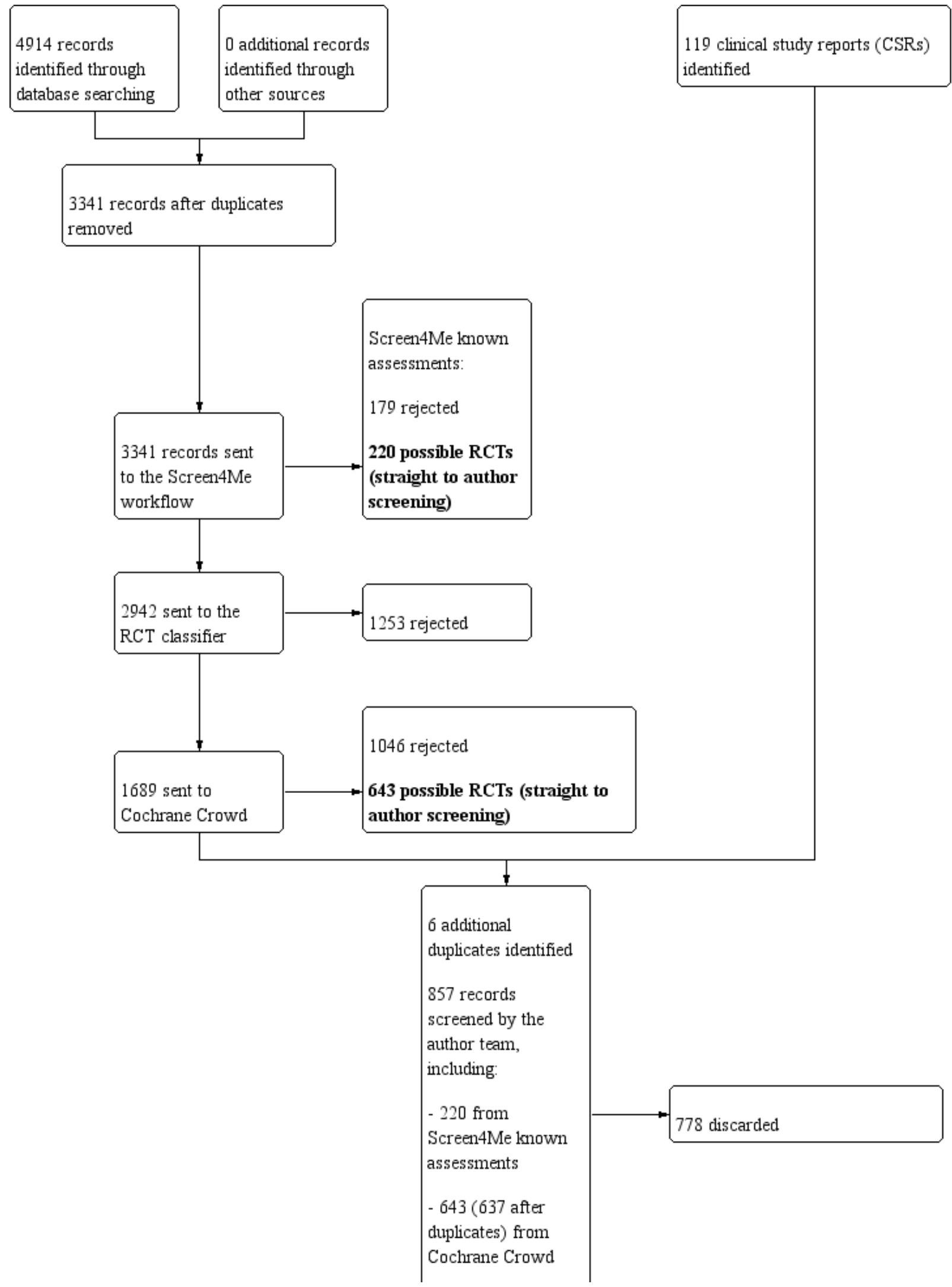


Figure 1. (Continued)

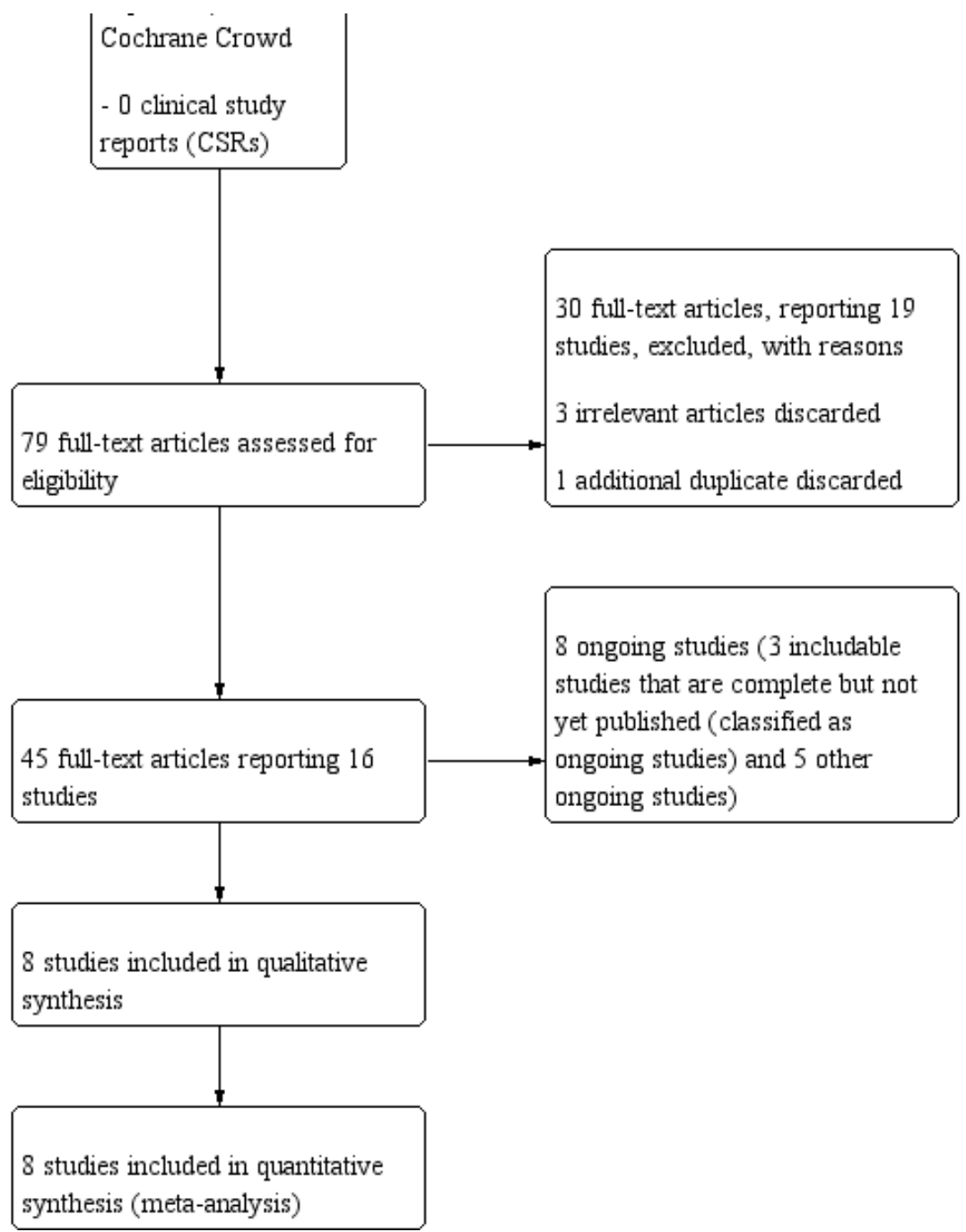

Possible RCTs

Rejected

\begin{tabular}{llc}
\hline Known assessments & 220 & 179 \\
\hline RCT classifier & & 1253 \\
\hline Cochrane Crowd & 643 & 1046 \\
\hline Total & $\mathbf{8 6 3}$ & $\mathbf{2 4 7 8}$ \\
\hline
\end{tabular}

We subsequently identified six additional duplicates, leaving 857 references to screen.

For further details of this process please see Selection of studies in the Methods section.

We screened the title and abstracts of the remaining 857 references. We discarded 778 references and assessed 79 full-text articles. We discarded three additional references at the full-text screening stage and identified one additional duplicate. We excluded 30 of these references (19 studies) with reasons recorded in the review (see Excluded studies).

We included eight completed studies, where results were available (31 references) (Bachert 2016; Bachert 2017; Gevaert 2011; Gevaert 2013; LIBERTY SINUS 24; LIBERTY SINUS 52; NCT01066104; Pinto 2010). NCT01066104 is an unpublished study 
(no journal publications or abstracts found), but the results of the study were available on the clinicaltrials.gov website.

There is one reference to one study that completed in March 2017 where the results have not yet been published and no information on the findings are available on clinicaltrials.gov (NCT02772419). The study was conducted by Kyowa Kirin Co. Ltd. The company confirmed on 7 January 2019 that the study is complete and that they are considering publication of the results. We requested access to the study results or clinical study report on 7 January 2019. The response from Kyowa Kirin is shown in Appendix 4. This study is classified as ongoing.

We identified another seven studies (13 references) that we classified as ongoing. Five studies were due to be completed in December 2019 and during 2020 (NCT02799446; NCT03450083; NCT03614923; OSTRO; SYNAPSE). An additional two studies were completed in 2019 and are due to publish their results in 2020 (POLYP 1; POLYP 2).

See Characteristics of ongoing studies for further details of all eight studies.

A flow chart of study retrieval and selection is provided in Figure 1.

\section{Included studies}

We found a total of eight completed RCTs (Bachert 2016; Bachert 2017; Gevaert 2011; Gevaert 2013; LIBERTY SINUS 24; LIBERTY SINUS 52; NCT01066104; Pinto 2010). All the studies were sponsored or supported by industry.

A summary of key participant characteristics, interventions, comparison pairs and outcomes measured and reported is provided in Table 1.

\section{Study design}

All studies were double-blind RCTs and used a placebo. The shortest planned duration was eight weeks (Gevaert 2011), the longest was 52 weeks (LIBERTY SINUS 52). One study was stopped early and only had 14 participants (Pinto 2010). Some studies were phase II or proof of concept studies and had fewer than 30 patients in each treatment arm (Gevaert 2011; Gevaert 2013; NCT01066104; Pinto 2010).

\section{Participants}

A total of 986 participants were included. With the exception of two participants in one study (Pinto 2010), all the participants were adults with chronic rhinosinusitis with nasal polyps and a significant number of participants (43\% to $100 \%$ ) also had asthma as a co-morbidity.

\section{Interventions and comparisons}

Studies were available to evaluate three of our four proposed comparison pairs. (No studies assessed the comparison antiIL-13 plus intranasal steroids versus placebo/no treatment plus intranasal steroids). All studies compared a biologic against placebo and all participants received intranasal corticosteroids.
Comparison 1: Anti-IL-4Ra mAb versus placebo/no treatment (all receiving intranasal steroids)

Three RCTs (784 participants) investigated dupilumab $300 \mathrm{mg}$ versus placebo.

- LIBERTY SINUS 24 (276 participants) gave 300 mg (subcutaneous, SC) dupilumab every two weeks and followed up patients for 24 weeks.

- LIBERTY SINUS 52 (448 participants) randomised patients 1:1:1 into three arms (two dupilumab arms and one placebo arm): $300 \mathrm{mg}$ SC dupilumab every two weeks for 52 weeks, or $300 \mathrm{mg}$ SC dupilumab every two weeks for 24 weeks followed by 300 mg SC dupilumab every four weeks for another 28 weeks. The total period of follow-up was 52 weeks and results were reported for both week 24 and 52 . The study had prespecified that some of the data would be pooled across both studies and/or both treatment arms of dupilumab, and did not report the results of the individual trials separately. For the purpose of this review, we combined the results of the different dupilumab arms in the LIBERTY SINUS 52 study, but reported the results of SINUS-52 and SINUS-24 independently by using the data presented in trial registries whenever possible.

- Bachert 2016 (60 participants) gave a 500 mg SC loading dose of dupilumab followed by $300 \mathrm{mg}$ SC weekly for 15 weeks.

Comparison 2: Anti-IL-5 mAb versus placebo/no treatment (all receiving intranasal steroids)

Two RCTs were found for this comparison.

- Bachert 2017 (107 participants).

- Gevaert 2011 (30 participants).

Both studied mepolizumab 750 mg intravenously every four weeks for 24 weeks.

Comparison 3: Anti-IgE mAb versus placebo/no treatment (all receiving intranasal steroids)

Three very small studies were found.

- Gevaert 2013 (24 participants).

- NCT01066104 (27 participants).

- Pinto 2010 (14 participants).

All studied subcutaneous omalizumab, at a dose dependent on the participants' weight and other characteristics, every two or four weeks for between 16 weeks and six months.

\section{Outcomes}

\section{Health-related quality of life (HRQL), using validated disease- specific HRQL scores}

Most studies measured and reported the SNOT-22. Two did not: Gevaert 2011 and NCT01066104. SNOT-22 has a range of 0 to 110 and the minimal clinically important difference (MCID) is 8.9 points (Hopkins 2009).

\section{Disease severity, as measured by validated patient-reported symptom score (such as the CSS questionnaire or visual analogue} scales)

LIBERTY SINUS 24 used a 0 to $10 \mathrm{~cm}$ visual analogue scale (VAS) to measure overall (global) symptoms ("How troublesome are your symptoms?", 0 = "not troublesome", 10 = "worst thinkable 
troublesome"). Other studies either did not provide details or reported some variation in how the question was asked. Bachert 2017 reported using a VAS of 0 to 10 with the question, "How troublesome are your symptoms of nasal polyposis?", 0 = "not troublesome", 10 = "worst possible". These studies generally made reference to the recommendation in EPOS 2007 to use a VAS, but did not report whether or not the format or wording of the questions they used in the trials had been validated.

Other measures such as "total symptom score" with a scale range of 0 to 9 points were used by some studies. However, this scale only measured symptoms of rhinitis (posterior and anterior rhinorrhoea) and nasal blockage rather than the overall symptom score of chronic rhinosinusitis, and other individual symptom scores, and there was no evidence of validation. Data from these scales, and on those relating to specific, individual symptoms, are not considered in our meta-analysis as they are not global symptom scores.

\section{Severe adverse events}

Most studies used the definition of treatment-emergent severe adverse events, where the events and participants were accounted for according to the treatment actually received (rather than by randomised group) and at least one dose was taken.

\section{Avoidance of surgery}

A few studies attempted to measure the degree of improvement (or non-improvement) experienced by participants, by identifying those participants who required some form of surgery to alleviate their symptoms. This took the form of determining the number of patients who required some form of 'rescue surgery', or the number of patients who met (or no longer met) the criteria for surgery. There are many issues and potential risks of bias associated with this measure. Table 2 summarises information for each included study about (a) whether or not the eligibility for surgery was defined at randomisation, and (b) in studies where the need for surgery was an 'outcome', what were the criteria for surgery in those circumstances?

In the two largest studies (724 participants), no specific criteria were given; it was stated that surgery was performed "when there was worsening of signs and/or symptoms during the study" (LIBERTY SINUS 24; LIBERTY SINUS 52).

In Bachert 2017, a set of criteria was used at randomisation and a different set at the trial's endpoint, to determine "eligibility for surgery". The criteria used were hypothetical; it is unclear how many participants were offered or underwent surgery. Moreover, whether or not these criteria correlate with actual patients' decisions to accept (and undergo) surgery (if offered) is unclear. It is also uncertain whether patients fulfilling these criteria would actually benefit from surgery (i.e. whether surgery is appropriate in these cases).

Therefore, although we identified a number of attempts by trialists to provide an indicator of whether biologics could reduce the need for surgery in patients, none of the studies used a validated method that can provide conclusive answers.

\section{5a. Extent of disease: endoscopic score}

A number of studies reported using "endoscopic nasal polyps score" (NPS) or total polyps score (TPS) and referenced Gevaert 2013, whereas the protocol for Bachert 2016 referenced a nonrelated paper. These had the same scoring system, utilising the total scores from both sides (bilateral, range 0 to 8). Unlike the Lund Kennedy and other scales with reported validation, these scales focused on the size of polyps, and not other factors such as the presence of inflammation and secretions/mucus.

Table: Scoring system for endoscopic nasal polyps score (NPS), or total polyps score (TPS)

\begin{tabular}{ll}
\hline Polyp score & Polyp size \\
\hline 0 & No polyps \\
\hline 1 & Small polyps in the middle meatus not reaching below the inferior border of the middle turbinate \\
\hline 2 & Polyps reaching below the lower border of the middle turbinate \\
\hline 3 & Large polyps reaching the lower border of the inferior turbinate or polyps medial to the middle \\
\hline 4 & turbinate \\
\hline
\end{tabular}

\section{5b. Extent of disease: computerised tomography (CT) scan score}

All studies (other than Bachert 2017) used the Lund Mackay score.

\section{Health-related quality of life (HRQL), using generic HRQL scores}

Generic health-related quality of life data were available from five studies. Data on the overall health status measured using the EQ-5D visual analogue scale were commonly reported and were used in our meta-analysis. A minimal clinically important difference (MCID) of 8 points has been reported by Hoehle 2019. Data from studies using the SF-36 are reported narratively, as incompleteness of the information did not allow data analysis.

\section{Adverse effects: nasopharyngitis, including sore throat}

Most studies reported this outcome. 


\section{Excluded studies}

We excluded 19 studies (30 references) after reviewing the full text. Further details of the reasons for exclusion can be found in the Characteristics of excluded studies table.

We excluded five studies due to the population (Castro 2011; Liberty Asthma Quest; MUSCA; NCT01285323; NCT02170337). NCT01285323 and MUSCA were in asthma patients. NCT02170337 was a safety study in healthy patients. Liberty Asthma Quest and Castro 2011 were studies in asthma patients with a subset of chronic rhinosinusitis patients. The chronic rhinosinusitis patients did not meet our inclusion criteria.

We excluded one study due to the intervention (Gevaert 2006). In this safety study a single dose of biologic was given, rather than a course of treatment.
We excluded 12 studies that were not RCTs (Boguniewicz 2019; De Schryver 2015; Gevaert 2008; Gonzalez-Diaz 2014; Hellings 2017; Laidlaw 2019; Naclerio 2017; NCT02743871; Perez De Llano 2018; Tajiri 2013; Zangrilli 2019).

Two studies were withdrawn (NCT00603785; NCT02734849).

\section{Risk of bias in included studies}

We included eight studies in this review. Overall the risk of bias was low or unclear for most domains.

See Figure 2 for the 'Risk of bias' graph (our judgements about each risk of bias item presented as percentages across all included studies) and Figure 3 for the 'Risk of bias' summary (our judgements about each risk of bias item for each included study).

\section{Figure 2. 'Risk of bias graph': review authors' judgements about each risk of bias item presented as percentages across all included studies.}

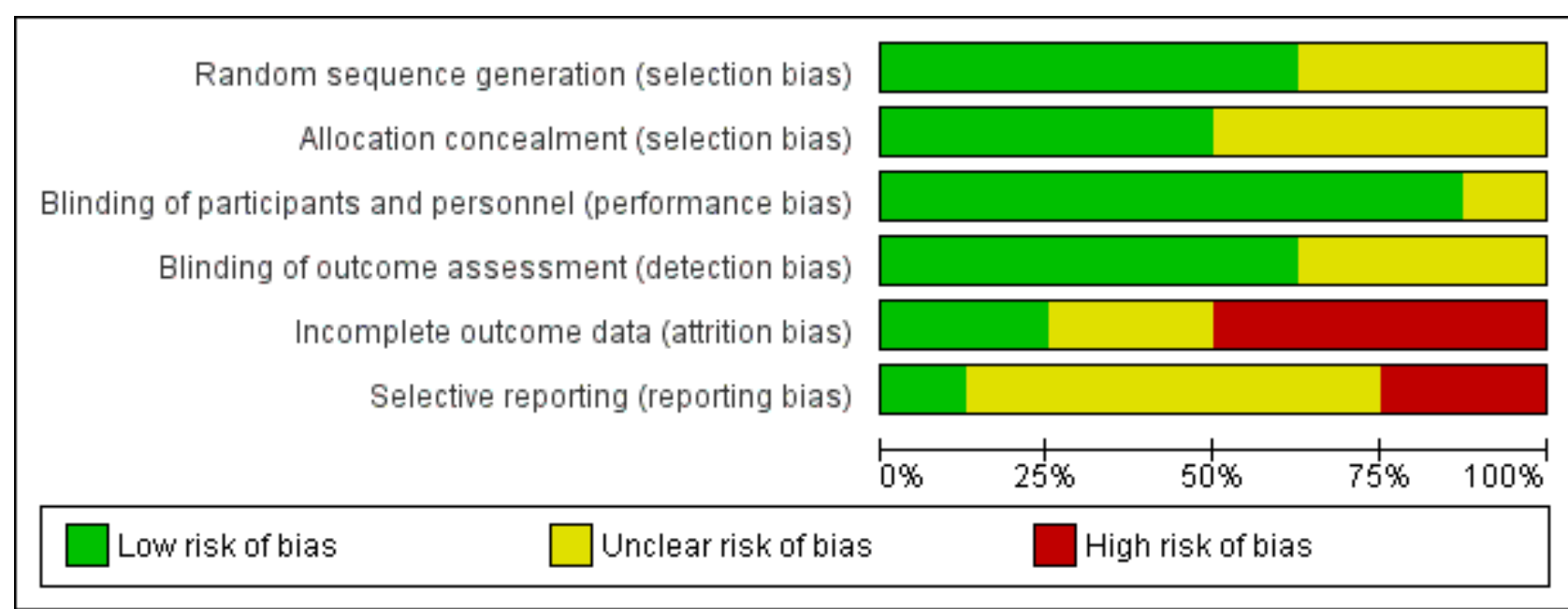


Figure 3. 'Risk of bias' summary: review authors' judgements about each risk of bias item for each included study.

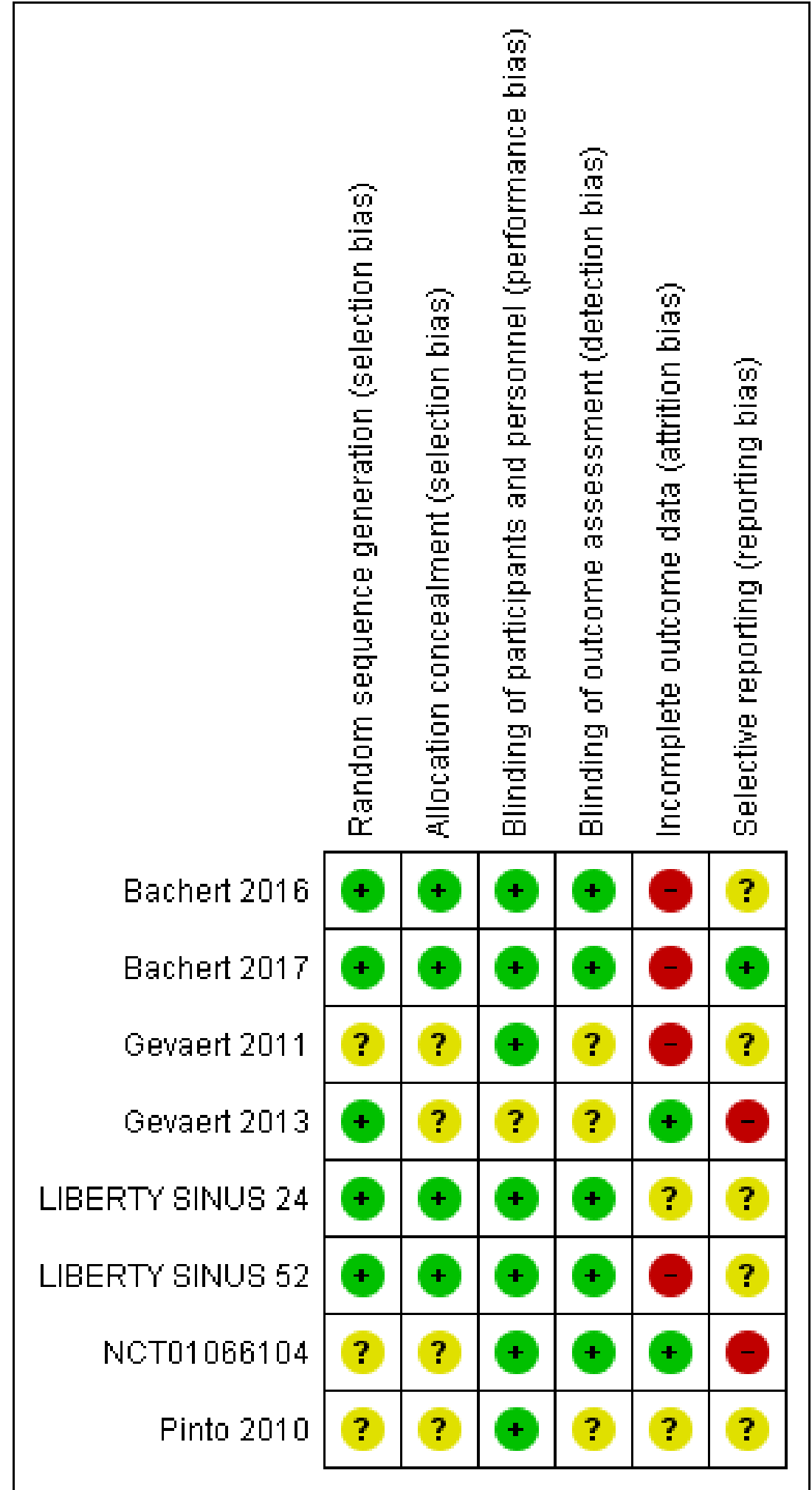

\section{Allocation}

The risk of selection bias was low or unclear in the majority of studies. We considered the risk of bias to be low for both random sequence generation and allocation concealment in four studies
(Bachert 2016; Bachert 2017; LIBERTY SINUS 24; LIBERTY SINUS 52), and the risk in both of these domains to be unclear for three studies (Gevaert 2011; NCT01066104; Pinto 2010). We considered the Gevaert 2013 study to be at low risk of bias for random sequence 
generation, but at high risk for allocation concealment, because a randomisation list was used.

\section{Blinding}

We considered seven of the eight studies to be at low risk of performance bias, since all participants and personnel were blind to treatment allocation. Both the investigator and participants were blinded in the Gevaert 2013 study, but it is not clear whether or not the study personnel were also blind. We therefore marked this domain as being at unclear risk of bias.

In five of the studies it was clear that people who were blind to treatment allocation assessed outcomes, so we considered these to be at low risk of detection bias (Bachert 2016; Bachert 2017; LIBERTY SINUS 24; LIBERTY SINUS 52; NCT01066104). We considered the remaining three studies to be at unclear risk of bias (Gevaert 2011; Gevaert 2013; Pinto 2010). Although Gevaert 2013 and Pinto 2010 mentioned that the CT scans were read by blinded assessors, it was not clear whether or not the nasal endoscopy outcome assessment was blind.

\section{Incomplete outcome data}

We assessed four of the studies to be at high risk of attrition bias (Bachert 2016; Bachert 2017; Gevaert 2011; LIBERTY SINUS 52), mostly due to high rates of discontinuation in these small studies. We assessed LIBERTY SINUS 52 to be at high risk because, although the investigators used a last observation carried forward (LOCF) imputation method, there were proportionally more discontinuations in the placebo arm. We assessed Gevaert 2013 and NCT01066104 to be at low risk of attrition bias, and considered LIBERTY SINUS 24 and Pinto 2010 to be at unclear risk of bias for this domain.

\section{Selective reporting}

We only considered one of the studies to be at low risk of selective reporting (Bachert 2017). There were differences between the NCT trial registration and reported outcomes for Gevaert 2013 and NCT01066104, so we assessed these to be at high risk of reporting bias. We found the other trials to be at unclear risk of reporting bias.

\section{Other potential sources of bias}

There are concerns about whether or not appropriate and validated tools were used for some outcomes. None of the studies reported using validated methods for their endoscopic scoring systems. All of the studies either did not provide details of the method used or had reported using a scoring system that took into account only the size of the polyps and we did not find any references to the validation of this system. Similarly, whilst many studies reported using a VAS for overall symptom score, they made no reference to validation. Although a VAS is a well-used type of scale, its validity needs to be confirmed in each specific population and for each outcome measured; factors such as the clarity of questions and the definition used for the 'best' and 'worst' points in the scale could affect a scale's validity.

The assessment of 'avoidance of surgery' (outcome 4 above) is fraught with difficulty; there is a high risk of bias in the included studies. Only a small number of studies defined eligibility for surgery at baseline. However, these studies did not use the same criteria for assessment of surgical eligibility at the trial's endpoint. Moreover, there is an absence of generally accepted or validated criteria as to what constitutes a situation which is 'severe' enough for patients to be willing to undergo surgery, or to benefit from it. Therefore, it is particularly unclear how these criteria were determined and/or the basis on which criteria were changed between entry and the endpoint of a study.

In those studies without any predefined or explicit criteria for surgery, it is even less clear how decisions were made to offer 'rescue surgery'. See Table 2 for further details.

\section{Effects of interventions}

See: Summary of findings for the main comparison Anti-IL-4Ra $\mathrm{mAb}$ (dupilumab) compared to placebo (on top of topical steroids) for chronic rhinosinusitis; Summary of findings 2 Anti-IL-5 mAb (mepolizumab) compared to placebo (on top of topical steroids) for chronic rhinosinusitis; Summary of findings 3 Anti-IgE mAb (omalizumab) compared to placebo (on top of topical steroids) for chronic rhinosinusitis

\section{Comparison 1: Anti-IL-4Ra mAb plus intranasal steroids versus} placebo/no treatment plus intranasal steroids

Three studies (784 participants) investigated dupilumab (Bachert 2016; LIBERTY SINUS 24; LIBERTY SINUS 52). See Summary of findings for the main comparison.

\section{Health-related quality of life, using validated disease-specific health-related quality of life scores}

Disease-specific health-related quality of life was measured with the Sino-Nasal Outcome Test-22 (SNOT-22, range 0 to 110, minimal clinically important difference (MCID) 8.9 points).

At 24 weeks, the SNOT-22 score was 19.61 points lower (better) in participants who received dupilumab (mean difference (MD) $-19.61,95 \%$ confidence interval (CI) -22.54 to $-16.69 ; 3$ studies; 784 participants; $I^{2}=0 \%$; high-certainty evidence; Analysis 1.1 ).

This effect was also seen at 52 weeks (MD $-22.38,95 \% \mathrm{Cl}-27.10$ to -17.66 ; 1 study; 303 participants), but the certainty of evidence is moderate due to imprecision (Analysis 1.1).

\section{Disease severity, as measured by validated patient-reported symptom score}

All of the studies used a 0 to $10 \mathrm{~cm}$ visual analogue scale (VAS) score to measure overall chronic rhinosinusitis symptoms. For the LIBERTY SINUS 24 and LIBERTY SINUS 52 studies (724 participants), the question asked was "How troublesome are your symptoms?". We found no evidence to indicate that this tool has been validated.

The pooled mean difference is -3.00 favouring the groups receiving dupilumab $\left(95 \% \mathrm{Cl}-3.47\right.$ to $-2.53 ; 3$ studies; 784 participants; $\mathrm{I}^{2}=$ $0 \%$; moderate-certainty evidence; Analysis 1.2 ). This is likely to be clinically significant.

\section{Serious adverse events}

The incidence of serious adverse events was measured over different periods: up to 16 weeks in Bachert 2016, 24 weeks in LIBERTY SINUS 24 and 52 weeks in LIBERTY SINUS 52. The risk seems to be lower in the treatment group (risk ratio (RR) 0.45 , $95 \% \mathrm{Cl} 0.28$ to $0.75 ; 3$ studies; 782 participants; $12=0 \%$; lowcertainty evidence; Analysis 1.3). There were discrepancies in the 
numbers reported in the different publications reporting the results of LIBERTY SINUS 24. Therefore, we used the data that matched those reported in clinicaltrials.gov in this analysis.

\section{Avoidance of surgery}

Two studies reported the number of participants requiring "nasal polyps surgery (actual or planned) during the treatment period". The proportion may be lower in the groups that received dupilumab (RR $0.17,95 \% \mathrm{Cl} 0.05$ to $0.52 ; 2$ studies; 725 participants; $\mathrm{R}=28 \%$; moderate-certainty evidence; Analysis 1.4). However, between baseline and endpoint there were changes in the criteria that determined whether or not a participant qualified for surgery. It was unclear how many qualified for surgery compared with how many actually received surgery, and the specific factors that determined whether or not a patient received 'rescue' surgery during follow-up. See Table 2 for more details on how this outcome was measured.

\section{5a. Extent of disease: endoscopy score}

All studies used a nasal polyps score, which summed the scores for both nostrils ( 0 to 8 points; $0=$ no polyp, $4=$ large polyps, for each nostril, with a lower score indicating smaller-sized polyps). The differences between the intervention arms were large (Cohen's effect size $>0.7=$ large effect), favouring the dupilumab group.

At 24 weeks follow-up the mean difference was $-1.80(95 \% \mathrm{Cl}$ -2.25 to -1.35 ; 3 studies; 784 participants; $\left.\right|^{2}=65 \%$; moderatecertainty evidence; Analysis 1.5), with a corresponding effect size of standardised mean difference (SMD) -1.05 (95\% Cl -1.29 to -0.82$)$. We found no evidence to indicate that this scoring system has been validated.

At 52 weeks, the mean difference was $-2.34(95 \% \mathrm{Cl}-2.77$ to -1.91 ; 1 study; 303 participants; low-certainty evidence; Analysis 1.5), and the corresponding effect size was SMD $-1.24(95 \% \mathrm{Cl}-1.48$ to -0.99$)$.

\section{5b. Extent of disease: computerised tomography (CT) scan score}

We pooled data from 16 weeks to 52 weeks as data were only available from one time point from each study.

The changes in the extent of disease were evaluated using a CT scan and scored using the Lund Mackay scale (0 to 24, higher = worse). The mean difference was $-7.00(95 \% \mathrm{Cl}-9.61$ to $-4.39 ; 3$ studies; 784 participants; $\left.\right|^{2}=92 \%$; high-certainty evidence; Analysis 1.6), showing a large effect favouring the dupilumab group. The corresponding SMD was $-1.50(95 \% \mathrm{Cl}-1.84$ to -1.15 ; Cohen's effect size $>0.7=$ large effect). We considered the certainty of the evidence to be high despite the large $1^{2}$ value; there is no inconsistency in terms of direction or size of effects between the three studies.

\section{Health-related quality of life, using generic health-related quality of life scores}

Two studies used the EQ-5D visual analogue scale (0 to 100, higher $=$ better) to measure the change in generic health-related quality of life (overall health state). The pooled MD of two studies was 8.59 points $\left(95 \% \mathrm{Cl} 5.31\right.$ to $11.86 ; 2$ studies; 706 participants; $\mathrm{I}^{2}=100 \%$; moderate-certainty evidence; Analysis 1.7). This effect size is similar to the size of the MCID ( 8 points, as suggested by Hoehle 2019) and therefore there is probably a clinically important improvement in this outcome. We noted the high $\mathrm{I}^{2}$ value, however the two pooled studies are LIBERTY SINUS 24 and LIBERTY SINUS 52 and the direction of effect is the same in both studies despite the differing time periods for outcome assessment ( 24 weeks and 52 weeks), therefore we considered this to be more a statistical quirk than an issue of concern.

\section{Adverse effects: nasopharyngitis, including sore throat}

The pooled results indicate that there is probably little or no difference in the risk of nasopharyngitis, but larger sample sizes are needed for a more precise estimate (RR $0.95,95 \% \mathrm{Cl} 0.72$ to 1.25 ; 3 studies; 783 participants; $12=0 \%$; low-certainty evidence; Analysis $1.8)$.

\section{Comparison 2: Anti-IL-5 mAb plus intranasal steroids versus placebo/no treatment plus intranasal steroids}

Two studies evaluated mepolizumab (Bachert 2017; Gevaert 2011). See Summary of findings 2.

\section{Health-related quality of life, using validated disease-specific health-related quality of life scores}

Data on disease-specific health-related quality of life as measured with the SNOT-22 were only available from one study (Bachert 2017: data from the EudraCT website). The mean difference of -13.26 lower (better) with mepolizumab $(95 \% \mathrm{Cl}-22.08$ to $-4.44 ; 1$ study; 105 participants; low-certainty evidence; Analysis 2.1) is greater than the MCID of 8.9 points.

\section{Disease severity, as measured by validated patient-reported symptom score}

Bachert 2017 reported using a VAS of 0 to 10 with the question "How troublesome are your symptoms of nasal polyposis?" $(0=$ "not troublesome", 10 = "worst possible"). The MD was $-2.03(95 \% \mathrm{Cl}$ -3.65 to $-0.41 ; 1$ study; 72 participants; very low-certainty evidence; Analysis 2.2). We are very uncertain about these data due to the very small sample size and the absence of evidence that a validated tool was used.

\section{Serious adverse events (SAEs)}

It is uncertain whether or not there is a difference in the risk of serious adverse events (RR 1.57, $95 \% \mathrm{Cl} 0.07$ to 35.46; 2 studies; 135 participants; $I^{2}=0 \%$; very low-certainty evidence; Analysis 2.3).

\section{Avoidance of surgery}

Each study applied different criteria for assessing the need for surgery (see Table 2). While Bachert 2017 reported the number of patients who still met the criteria for surgery at the end of trial, Gevaert 2011 reported the number that required surgery during the period of the trial. It is very uncertain whether or not the overall risk that patients still need surgery at the end of trial is lower in the mepolizumab group (RR $0.78,95 \% \mathrm{Cl} 0.64$ to $0.94 ; 2$ studies; 135 participants; 12 =0\%; very low-certainty evidence; Analysis 2.4).

\section{5a. Extent of disease: endoscopic score}

The mean difference in the change of the nasal polyps score was 1.23 points lower in the mepolizumab group (MD -1.23, 95\% - 1.79 to -0.68 ; 2 studies; 137 participants; $12=0 \%$; very low-certainty evidence; Analysis 2.5). This corresponds to a moderate effect size (SMD - $0.69,95 \%-1.04$ to -0.34 ; low-certainty evidence). We found no evidence to indicate that this scoring system has been validated. 


\section{5b. Extent of disease: computerised tomography (CT) scan score}

Gevaert 2011 did not report the numerical values of the CT scan scores, but stated that at week eight the scores "were not significantly different between groups". Bachert 2017 did not measure CT scan scores. The evidence for this outcome was of very low certainty.

\section{Health-related quality of life, using generic quality of life scores}

The mean difference on the EQ-5D visual analogue scale was 5.68 in one study $(95 \% \mathrm{Cl}-1.18$ to $12.54 ; 1$ study; 105 participants; lowcertainty evidence; Analysis 2.6), favouring the mepolizumab group (Bachert 2017). This difference is smaller than the MCID of 8 points.

\section{Adverse effects: nasopharyngitis, including sore throat}

There may be little or no difference in the risk of nasopharyngitis (RR 0.73 , 95\% 0.36 to 1.47 ; 2 studies; 135 participants; 12 = 0\%; lowcertainty evidence; Analysis 2.7).

\section{Comparison 3: Anti-IgE mAb plus intranasal steroids versus placebo/no treatment plus intranasal steroids}

We identified three very small studies evaluating omalizumab (Gevaert 2013; NCT01066104; Pinto 2010). See Summary of findings 3.

\section{Health-related quality of life, using validated disease-specific health-related quality of life scores}

A narrative summary was reported in Gevaert 2013 (24 participants): "On the basis of the 31-item Rhinosinusitis Outcome Measuring Instrument (RSOM-31), sleep $(P=0.03)$ and general symptoms $(P=0.01)$ showed a significant improvement in the omalizumab group, whereas in the placebo group no significant changes were seen".

Pinto 2010 reported that the median change in SNOT-20 score was -1.05 for the omalizumab group and -0.20 for the placebo group ( $P$ $<0.78$ for the difference between groups).

The evidence for this outcome was of very low certainty.

\section{Disease severity, as measured by validated patient-reported symptom score}

Pinto 2010 reported that "The median TNSS for each month did not vary between visits by analysis of variance for either group ( $P$ $>0.05$, all comparisons), with no significant net difference across treatments (omalizumab -1 , placebo $0, \mathrm{P}<0.21$ )".

\section{Serious adverse events (SAEs)}

Although all three studies collected data on severe adverse events, no event was reported (very low-certainty evidence). In total, the treatment groups across three studies had 35 participants, while the placebo group had 29 participants; this number is too small to properly assess severe adverse events (Analysis 3.1).

\section{Avoidance of surgery}

None of the studies reported this outcome.

\section{5a. Extent of disease: endoscopic score}

Two studies evaluated and reported nasal polyps scores $(0$ to 8 points, higher $=$ worse). Although the pooled mean difference of $-1.63\left(95 \% \mathrm{Cl}-3.73\right.$ to $0.47 ; 2$ studies; 47 participants; $\left.\mathrm{I}^{2}=81 \%\right)$ corresponds to a large effect size (SMD $-1.51,95 \% \mathrm{Cl}-4.22$ to 1.21 ; Analysis 3.2), there is inconsistency because the effect sizes seen in the two studies are very different. One study showed a large effect size with an SMD of nearly 3 (Gevaert 2013); in the other study both arms had similar scores (NCT01066104). The evidence for this outcome is of very low certainty.

Pinto 2010 reported that "There were no significant changes within in endoscopy scores for either group (data not shown). Net change across treatments were not significantly different (omalizumab 0 , placebo $-0.5, \mathrm{P}<0.58)$ ". There was no information about what scoring system was used or whether one or both sides of the nose were assessed and scored. The paper reported using a 0 - to 4-point score, but referenced a paper using a 0 - to 3-point scale.

\section{5b. Extent of disease: computerised tomography (CT) scan score}

Gevaert 2013 reported the Lund Mackay scores at the endpoint whereas NCT01066104 reported the percentage change compared to baseline using a modification of the Lund Mackay score (no reports of validation). In both studies, lower scores mean a better outcome for the patients. The observed pooled results correspond to a small effect size (SMD $-0.20,95 \% \mathrm{Cl}-1.55$ to $1.14 ; 2$ studies; 47 participants; $\left.\right|^{2}=80 \%$; Analysis 3.3).

Statistical heterogeneity is high and there are inconsistencies in the size and direction of effect. In the NCT01066104 study, the results favoured the placebo group, while in Gevaert 2013 they favoured the intervention group. The evidence for this outcome was of very low certainty.

\section{Health-related quality of life, using generic quality of life scores}

Two studies used the SF-36 to measure health-related quality of life. Pinto 2010 reported that "Across treatments, there were also no significant differences ( $P>0.05$, all comparisons) except for one domain, Vitality (omalizumab 9.4, placebo 12.5, $\mathrm{P}<$ 0.05)." Gevaert 2013 reported, "After 16 weeks, the Short-Form Health Questionnaire (SF-36) for physical health was significantly improved in the omalizumab group $(P=0.02)$ but not in the placebo group $(P=0.75)$. Unlike physical health, mental health did not significantly improve in either treatment group." The evidence for this outcome was of very low certainty.

\section{Adverse effects: nasopharyngitis, including sore throat}

No nasopharyngitis was reported in any of the three studies in either intervention arm. The total sample size (35 participants in the intervention group, 29 in the placebo group) is probably too small to detect adverse events (very low-certainty evidence).

\section{DISCUSSION}

\section{Summary of main results}

We identified randomised controlled trials (RCTs) evaluating the effectiveness of three different drugs, representing three different types of monoclonal antibodies. These were dupilumab (an anti- 
IL-4Ra mAb), mepolizumab (an anti-IL-5 mAb) and omalizumab (an anti-IgE $m A b)$.

The first two drugs were evaluated in adults with chronic rhinosinusitis and nasal polyps who were also using regular topical nasal steroids. In these patients, we found high-certainty evidence from three studies (with nearly 800 participants) that dupilumab improves disease-specific health-related quality of life (HRQL) compared to placebo, and reduces the extent of the disease as measured on a computerised tomography (CT) scan. Moderatecertainty evidence shows that it probably also improves symptoms, generic HRQL as measured by overall health status and size of polyps as measured by nasal polyp scores. It may reduce the need for further surgery but it is difficult to interpret the clinical implications of this finding due to methodological limitations. There is probably little or no difference in the risk of nasopharyngitis.

Mepolizumab has been evaluated in similar patients but the certainty of evidence is either low or very low. It may improve both disease-specific and generic HRQL, and improve nasal polyp scores. As with dupilumab, it may reduce the need for surgery, but there are important limitations of the methodology that limit the clinical interpretation of the data. There may be little or no difference in the risk of nasopharyngitis. It is very uncertain if there is a difference in the risk of serious adverse events.

We are very uncertain about the effects of omalizumab because the evidence is very limited and of low or very low certainty.

\section{Overall completeness and applicability of evidence}

There are four major limitations pertaining to the completeness and applicability of the evidence:

1. All but one study (Pinto 2010) recruited patients with moderate to severe chronic rhinosinusitis with nasal polyps, as defined by polyp size and need for systemic steroids and/or surgery, and at least half of the participants also had asthma as a comorbidity. Therefore, there is no evidence on whether or not patients with less severe disease (with or without nasal polyposis or asthma) would benefit as much or at all.

2. All studies were in adults. There are no data for children.

3. There is a lack of long-term evidence. Whilst treatment with biologics is arguably a lifetime commitment, there was only one study with a 52-week follow-up. It was not always possible to compare the mid-term (24-week) data with the longer-term data in this study. However, where data were published (SNOT-22 and endoscopy score) the effect size was maintained (LIBERTY SINUS 52).

4. The sample sizes were insufficient and the length of follow-up too short to comprehensively and adequately assess the risks of side effects.

\section{Quality of the evidence}

The primary reason for downgrading the quality of the available evidence was imprecision, where sample sizes were too small to provide a precise estimate.

In addition, the lack of evidence that validated scales or scoring systems were used was also a concern, especially for symptom scores and endoscopy scores. As in other studies found in this series of Cochrane Reviews, the lack of use of a globally validated symptom score scale, which focuses on overall disease severity, continues to be a problem. It is difficult to compare 'the overall improvement' of symptoms across trials or reviews if studies use different scales, with different weightings given to different types of symptoms. Although there have been improvements in methodology compared to previous studies, in the sense that studies attempted to use visual analogue scales, there was no evidence that these scales had been validated and that they are comparable across studies. In addition, many studies also used a scoring system for nasal endoscopy that only takes into account the size of polyps. There is no reference to how this scale has been validated against patient outcomes.

All but one study (Pinto 2010) focused (sometimes solely) on recruiting patients who had comorbid asthma and more severe nasal polyposis. However, notwithstanding this we did not further downgrade studies based on applicability.

It should also be noted that the evidence available is relatively short-term; only one study was conducted for more than six months. We did not downgrade the evidence for indirectness due to the relatively short follow-up.

\section{Potential biases in the review process}

None of the studies reported using a validated overall symptom score measure to assess changes in patients' symptom severity. Some studies reported specific types of chronic rhinosinusitis symptoms using different tools, for many of which there was no evidence of validation.

To provide the best possible picture of overall symptoms, we examined each reported tool carefully and used data from questions/questionnaires that asked about overall symptoms. We avoided using data from tools that only measured one or two specific symptoms of chronic rhinosinusitis. For example, we did not use data from the 'total symptom score' (TSS); this only measured symptoms of anterior and posterior rhinorrhoea and nasal blockage. The symptoms of loss of sense of smell and facial pain were not measured.

Whenever an overall symptom assessment was reported using a visual analogue scale, we recorded and used those data even though there were slight variations between studies in how the questions were worded.

\section{Agreements and disagreements with other studies or reviews}

No previous systematic reviews have included the two largest trials (LIBERTY SINUS 24; LIBERTY SINUS 52), published in November 2019, which evaluate the effects of dupilumab and together contribute 724 or 986 participants to the current review and metaanalysis.

A systematic review, Tsetsos 2018, reported five trials that we also included in this Cochrane Review (Bachert 2016; Bachert 2017; Gevaert 2011; Gevaert 2013; Pinto 2010) and one that we excluded (Gevaert 2006). Their primary outcome was total nasal endoscopic polyp score. They did not perform a meta-analysis.

Rivero 2017 included randomised and non-randomised studies in their systematic review and meta-analysis. Three of our included 
studies were also included in their review (Gevaert 2011; Gevaert 2013; Pinto 2010). Nasal polyp score was their primary outcome of interest. The differences in the study types means that is not appropriate to compare the results of their meta-analyses with those in this review.

An earlier systematic review, Hong 2015, only identified two RCTs (Gevaert 2013; Pinto 2010).

In summary, there are no systematic reviews or meta-analyses with which it is appropriate to compare the results of the present review.

\section{AUTHORS' CONCLUSIONS}

\section{Implications for practice}

Patients with chronic rhinosinusitis, with and without nasal polyps, often need long-term treatment. Many have surgery and revision surgery is common, with a 10-year revision rate in excess of $15 \%$ in a large population study (Smith 2019), and with over $50 \%$ of patients in a UK epidemiological study reporting previous surgery for chronic rhinosinusitis with nasal polyps (CRSwNP) (Philpott 2015). Patients with chronic rhinosinusitis with nasal polyps and comorbid asthma are at a higher risk of undergoing revision surgery, and many of these patients experience poor symptom control, the need for repeated systemic steroids and multiple surgeries. The majority of trials included in this review have selected patients with severe chronic rhinosinusitis with nasal polyps, as defined by polyp size and the need for systemic steroids and/or surgery, both of which carry a risk of significant adverse effects. These severely affected patients, who had effectively failed other treatment options, experienced significant improvements in health-related quality of life and reduced disease severity on radiological imaging. Importantly, there does not appear to be any increased risk of severe adverse events, at least in the short term. This has the potential, therefore, to be a 'game-changer' in the management of patients with severe disease, allowing them to avoid other treatments associated with higher risk.

We are currently unable to predict which patients will respond to biologics. The included studies report response rates between $50 \%$ and $70 \%$, and therefore not all patients will respond to these drugs. Nor is it clear how to choose the optimum biologic, and when to consider these drugs, particularly with regards to using them before or after surgery. We also do not know if these drugs are effective in patients with less severe disease so we must highlight the potentially limited generalisability of the reported findings to the wider population of patients with chronic rhinosinusitis.

Finally, although not considered in this review, currently these drugs are high-cost compared to conventional treatment with topical and systemic corticosteroids and surgery, and patients require ongoing treatment with them. Both health economic analysis and long-term effectiveness studies are required to help guide usage and balance the societal costs with the needs of individual patients as the costs of long-term treatment with biologics, at current drug price levels, will be substantial.

\section{Implications for research}

Trials continue to use a heterogenous group of outcomes and do not include the recently published core outcome set for chronic rhinosinusitis (Hopkins 2018). There is an urgent need to validate or refine the nasal polyp scoring system and to ensure that it is uniformly applied.

Further data analysis is required to report response rates and future trials should aim to identify biomarkers that will predict response and allow selection of the 'best' biologic in each individual patient, in what is likely to be a growing field of different biologics. It will also be important to evaluate response rates and effectiveness in different subgroups as outlined above.

In many healthcare settings, the current high cost of biologics, and the fact that their efficacy has only been demonstrated in severely affected patients, will likely limit their use only to these patients at the present time. Studies are required to evaluate their effectiveness in patients with a less severe disease burden and in patients with chronic rhinosinusitis without nasal polyps. We also need comparative studies to evaluate different biologics and to compare them with conventional therapies, as well as studies that evaluate the optimum timing of use of different interventions. For example, studies are needed to determine if biologics can be disease-modifying if given early in the disease process (and therefore may be discontinued without relapse) or whether ongoing usage is required regardless of when the treatment is initiated. Also, studies are required to determine whether there is any difference in effectiveness if biologics are used before or after surgery. Finally, long-term observational studies are required to determine if biologics lose effectiveness over time, for example due to the development of neutralising antibodies, or whether there are any late adverse events.

\section{ACKN OWLEDGEMENTS}

This project was supported by the National Institute for Health Research, via Cochrane Infrastructure, Cochrane Programme Grant or Cochrane Incentive funding to Cochrane ENT. The views and opinions expressed therein are those of the authors and do not necessarily reflect those of the Systematic Reviews Programme, NIHR, NHS or the Department of Health.

We would like to thank Andrea Takeda and Katie Webster, Systematic Reviewers with Cochrane ENT, for their work and guidance, which facilitated the completion of this review.

We are grateful to Dr Hugo Farne and Professor Wytske Fokkens for clinical peer review of the draft protocol, and to Dr Rodney Schlosser for clinical peer review of the draft review. Thank you to Joanne Brooker and Britta Tendal from the Living Evidence Network for peer review of the living systematic review methodology and to Justin Clark, Information Specialist with Cochrane Acute Respiratory Infections and the Living Evidence Network, for providing peer review comments on the draft search methods. Our thanks also to Joan Blakley for her consumer refereeing at both protocol and review stage, which helped to improve our drafts.

We would also like to thank Julian Elliot, Anneliese Synnot, Ella Flemyng, Kerry Dwan, Toby Lasserson and Julian Higgins for their advice on living systematic reviews, inclusion of clinical study reports and ROB-2, and Nuala Livingstone from the Cochrane MOSS Network for her input.

We are grateful to the 47 Cochrane Crowd screeners for screening 1689 records to identify 643 possible RCTs, and reject 1046 
references as not RCTs. We are particularly grateful to Nicole Edworthy, Kamal Sharif, Leah Mohandas, Abhijna Vithal Yergolkar, Julie Cattini, Anna Resolver, Rachel Playforth, Miguel Rodriguez-
Rubio, Brian Duncan, Dhasarathi Kumar, Abhijit Dutta, Anna NoelStorr, Karen Ma, Diana De la Torre, Nikolaos Sideris and Emmet Farragher for screening more than 200 records each. 


\section{R E F E R E N C E S}

\section{References to studies included in this review}

\section{Bachert 2016 \{published and unpublished data\}}

Bachert C, Hellings P, Mullol J, Hamilos D, Naclerio R, Joish VN, et al. Dupilumab improves patient-reported outcomes in chronic sinusitis with nasal polyps patients with comorbid asthma: results from a phase 2a trial. European Respiratory Journal 2016;48(Suppl 60):OA251. [5632585]

Bachert C, Hellings PW, Mullol J, Hamilos DL, Gevaert P, Naclerio RM, et al. Dupilumab improves health-related quality of life in patients with chronic rhinosinusitis with nasal polyposis. Allergy 2019;71:12. [PUBMED: 31306495]

Bachert C, Hellings PW, Mullol J, Naclerio RM, Hamilos DL, Gevaert P, et al. Atopic comorbidities and biomarkers of type 2 inflammation in patients with chronic rhinosinusitis with nasal polyposis (CRSwNP) who failed intranasal corticosteroids. Journal of Allergy and Clinical Immunology 2018;141(2 Suppl 1):AB90. [7912807]

* Bachert C, Mannent L, Naclerio RM, Mullol J, Ferguson BJ, Gevaert P, et al. Effect of subcutaneous dupilumab on nasal polyp burden in patients with chronic sinusitis and nasal polyposis: a randomized clinical trial. JAMA 2016;315(5):469-79. [1861626; PUBMED: 26836729]

Bachert C, Mannent L, Naclerio RM, Mullol J, Ferguson BJ, Jiao $L$, et al. Dupilumab in chronic sinusitis with nasal polyposis, with and without asthma. Allergy 2015;70:107. [1866543]

Bachert C, Naclerio R, Hellings P, Guillonneau S, Taniou C, Maroni J, et al. Dupilumab improves mental health measures in patients with chronic rhinosinusitis and nasal polyposis (CRSWNP). European Respiratory Journal 2018;52(Suppl 62):PA5004. [10534714]

EUCTR2013-001803-35-BE. An evaluation of dupilumab in patients with nasal polyposis and chronic symptoms of sinusitis [A randomized, double-blind, phase 2, placebo controlled, 2 arm study to evaluate dupilumab in patients with bilateral nasal polyposis and chronic symptoms of sinusitis]. http://apps.who.int/trialsearch/Trial2.aspx? TrialID=EUCTR2013-001803-35-BE (first received 30 May 2013). [1706303]

Jonstam K, Swanson BN, Mannent LP, Cardell LO, Tian N, Wang Y, et al. Dupilumab reduces local type 2 pro-inflammatory biomarkers in chronic rhinosinusitis with nasal polyposis. Allergy 2019;74(4):743-52. [9965908; PUBMED: 30488542]

NCT01920893. An evaluation of dupilumab in patients with nasal polyposis and chronic symptoms of sinusitis [A randomized, double-blind, phase 2, placebo controlled, 2 arm study to evaluate dupilumab in patients with bilateral nasal polyposis and chronic symptoms of sinusitis]. https:// clinicaltrials.gov/show/NCT01920893 (first received 12 August 2013). [1689333]

Schneider JS. Subcutaneous dupilumab and mometasone furoate nasal spray for chronic rhinosinusitis with polyps. JAMA
Otolaryngology - Head and Neck Surgery 2016;142(7):698-9. [4435569; PUBMED: 27258602]

Swanson BN, Mannent L, Hamilton JD, Zhang D, Tian N, Wang Y, et al. The effect of dupilumab on biomarkers in the peripheral blood and nasal secretions in the treatment of chronic sinusitis with nasal polyposis. Inflammation Research 2015;64(2 Suppl 1):S118-9. [1838460]

Bachert 2017 \{published and unpublished data\}

* Bachert C, Sousa AR, Lund VJ, Scadding GK, Gevaert P, Nasser $S$, et al. Reduced need for surgery in severe nasal polyposis with mepolizumab: randomized trial. Journal of Allergy and Clinical Immunology 2017;140(4):1024-31.e14. [7111780; PUBMED: 28687232]

EUCTR2008-003772-21-NL. A two-part, randomised, doubleblind, placebo controlled, multi-center study to investigate the use of mepolizumab (SB-240563) in reducing the need for surgery in subjects with severe bilateral nasal polyposis. https:// www.clinicaltrialsregister.eu/ctr-search/trial/2008-003772-21/ NL (first received 27 February 2009). [1743659]

GlaxoSmithKline. A randomised, double blind, placebo controlled, multi-centre study to investigate the use of mepolizumab (SB240563) in reducing the need for surgery in subjects with severe bilateral nasal polyposis. GSK Clinical Study Report. [MPP111782]

NCT01362244. A randomised, double-blind, placebo controlled, multi-center study to investigate the use of mepolizumab (Sb-240563) in reducing the need for surgery in subjects with severe bilateral nasal polyposis. https://clinicaltrials.gov/show/ nct01362244 (first received 26 May 2011). [1629203]

\section{Gevaert 2011 \{published data only\}}

* Gevaert P, Van Bruaene N, Cattaert T, Van Steen K, Van Zele T, Acke F, et al. Mepolizumab, a humanized anti-IL-5 mAb, as a treatment option for severe nasal polyposis. Journal of Allergy and Clinical Immunology 2011;128(5):989-95.e1-8. [DOI: 10.1016/j.jaci.2011.07.056; 1601924; PUBMED: 21958585]

\section{Gevaert 2013 \{published and unpublished data\}}

EUCTR2006-003524-11-BE. Clinical and biological effects of anti-IgE (omalizumab) in patients with bilateral nasal polyposis and asthma [A randomized, double-blind, phase 2, placebo controlled, 2 arm study to evaluate dupilumab in patients with bilateral nasal polyposis and chronic symptoms of sinusitis]. http://apps.who.int/trialsearch/Trial2.aspx? TrialID=EUCTR2006-003524-11-BE (first received 11 August 2006). [1770331]

Gevaert P, Calus L, Van Zele T, Blomme K, De Ruyck N, Bauters W, et al. Omalizumab is effective in allergic and nonallergic patients with nasal polyps and asthma. Journal of Allergy and Clinical Immunology 2012;129(2 Suppl 1):AB69. [1765640]

* Gevaert P, Calus L, Van Zele T, Blomme K, De Ruyck N, Bauters W, et al. Omalizumab is effective in allergic and nonallergic patients with nasal polyps and asthma. Journal of 
Allergy and Clinical Immunology 2013;131(1):110-6.e1. [1617401; PUBMED: 23021878]

NCT01393340. Clinical and biological effects of anti-lgE (omalizumab) in patients with bilateral nasal polyposis and asthma. https://clinicaltrials.gov/show/NCT01393340 (first received 13 July 2011). [1629212]

\section{LIBERTY SINUS 24 \{published and unpublished data\}}

* Bachert C, Han JK, Desrosiers M, Hellings PW, Amin N, Lee SE, et al. Efficacy and safety of dupilumab in patients with severe chronic rhinosinusitis with nasal polyps (LIBERTY NP SINUS-24 and LIBERTY NP SINUS-52): results from two multicentre, randomised, double-blind, placebo-controlled, parallel-group phase 3 trials. Lancet 2019;394(10209):1638-50. [12131774; PUBMED: 31543428$]$

EUCTR2015-003101-42-BG. Controlled clinical study of dupilumab in patients with nasal polyps [A randomized, 24week treatment, double-blind, placebo-controlled efficacy and safety study of dupilumab $300 \mathrm{mg}$ every other week, in patients with bilateral nasal polyposis on a background therapy with intranasal corticosteroids - SYNUS-24]. https://www.clinicaltrialsregister.eu/ctr-search/search? query=eudract_number:2015-003101-42 (first received 4 January 2017). [10716209]

Han JK, Bachert C, Desrosiers M, Laidlaw TM, Hopkins C, Fokkens WJ, et al. Efficacy and safety of dupilumab in patients with chronic rhinosinusitis with nasal polyps: results from the randomized phase 3 sinus-24 study. Journal of Allergy and Clinical Immunology 2019;143(2):AB422. [10639235]

NCT02912468. A controlled clinical study of dupilumab in patients with bilateral nasal polyps [A randomized, 24-week treatment, double-blind, placebo-controlled efficacy and safety study of dupilumab $300 \mathrm{mg}$ every other week, in patients with bilateral nasal polyposis on a background therapy with intranasal corticosteroids]. https://clinicaltrials.gov/show/ NCT02912468 (first received 23 September 2016). [6978608]

\section{LIBERTY SINUS 52 \{published and unpublished data\}}

Bachert C, Desrosiers M, Mullol J, Hellings PW, Cervin A, Sher L, et al. A randomized phase 3 study, sinus-52, evaluating the efficacy and safety of dupilumab in patients with severe chronic rhinosinusitis with nasal polyps. Journal of Allergy and Clinical Immunology 2019;143(2):AB433. [9965830]

* Bachert C, Han JK, Desrosiers M, Hellings PW, Amin N, Lee SE, et al. Efficacy and safety of dupilumab in patients with severe chronic rhinosinusitis with nasal polyps (LIBERTY NP SINUS-24 and LIBERTY NP SINUS-52): results from two multicentre, randomised, double-blind, placebo-controlled, parallel-group phase 3 trials. Lancet 2019;394(10209):1638-50. [12131774; PUBMED: 31543428]

Bachert C, Hellings PW, Mullol J, Naclerio RM, Chao J, Amin N, et al. Dupilumab improves patient-reported outcomes in patients with chronic rhinosinusitis with nasal polyps and comorbid asthma. Journal of Allergy and Clinical Immunology: In Practice 2019;7(7):2447-9.e2. [12043116; PUBMED: 30928658]
EUCTR2015-001314-10-ES. Controlled clinical study of dupilumab in patients with nasal polyps [A randomized, double-blind, 52-week, placebo controlled efficacy and safety study of dupilumab, in patients with bilateral nasal polyposis on a background therapy with intranasal corticosteroids]. https://www.clinicaltrialsregister.eu/ctr-search/search? query=eudract_number:2015-001314-10 (first received 10 October 2016). [10715675]

NCT02898454. Controlled clinical study of dupilumab in patients with nasal polyps [A randomized, double-blind, 52-week, placebo controlled efficacy and safety study of dupilumab, in patients with bilateral nasal polyposis on a background therapy with intranasal corticosteroids]. https:// clinicaltrials.gov/show/NCT02898454 (first received 13 September 2016). [6978611]

\section{NCT01066104 \{unpublished data only\}}

NCT01066104. Subcutaneous omalizumab for treatment of chronic rhinosinusitis with nasal polyposis. https:// clinicaltrials.gov/show/nct01066104 2009. [CRS: 1647960]

\section{Pinto 2010 \{published data only\}}

Mehta NJ, Pinto J, de Tineo M, Baroody FM, Naclerio RM. A randomized, double-blind, placebo-controlled clinical trial of omalizumab for chronic rhinosinusitis. Journal of Allergy and Clinical Immunology 2009;123(2 Suppl 1):S201. [CRS: 1494226]

NCT00117611. Xolair in patients with chronic sinusitis [Effects of anti-IgE antibody omalizumab (Xolair) on patients with chronic sinusitis and a positive allergen test]. https://clinicaltrials.gov/ show/NCT00117611 2005. [CRS: 1770122]

* Pinto JM, Mehta N, DiTineo M, Wang J, Baroody FM, Naclerio RM. A randomized, double-blind, placebo-controlled trial of anti-IgE for chronic rhinosinusitis. Rhinology 2010;48(3):318-24. [CRS: 1569961; PUBMED: 21038023]

\section{References to studies excluded from this review}

Boguniewicz 2019 \{published data only\}

Boguniewicz M, Thaci D, Lio PA, Hultsch T, Rossi AB, Eckert L, et al. Dupilumab improves outcomes of concurrent asthma and chronic sino-nasal conditions in patients with atopic dermatitisa pooled analysis of four phase 3 studies (LIBERTY AD SOLO $1 \& 2$, CHRONOS, and CAFE). Journal of Allergy and Clinical Immunology 2019;143(2 Suppl):AB123. [CRS: 9965833]

\section{Castro 2011 \{published data only\}}

Castro M, Mathur S, Hargreave F, Boulet LP, Xie F, Young J, et al. Reslizumab for poorly controlled, eosinophilic asthma: a randomized, placebo-controlled study. American Journal of Respiratory and Critical Care Medicine 2011;184(10):1125-32. [CRS: 1602806; PUBMED: 21852542]

Mathur S, Castro M, Hargreave F, Xie F, Wilkins HJ, Henkel T, et al. Efficacy of reslizumab in patients with poorly controlled eosinophilic asthma: subgroup analysis of patients with nasal polyps [Abstract]. Journal of Allergy and Clinical Immunology 2011;127(2 Suppl 1):AB84. [CENTRAL: CN-00793509] 


\section{De Schryver 2015 \{published data only\}}

De Schryver E, Van Zele T, Bachert C, Gevaert P. Comparison of different medical treatment options for CRSwNP: doxycycline, methylprednisolone, mepolizumab and omalizumab. Allergy 2015;70:442. [CENTRAL: CN-01135998; CRS: 1866506; EMBASE: 72029693]

\section{Gevaert 2006 \{published data only\}}

* Gevaert P, Lang-Loidolt D, Lackner A, Stammberger H, Staudinger $\mathrm{H}$, Van Zele T, et al. Nasal IL-5 levels determine the response to anti-IL-5 treatment in patients with nasal polyps. Journal of Allergy and Clinical Immunology 2006;118(5):1133-41. [CENTRAL: CN-00573474; CRS: 1402690; EMBASE: 2006535588; PUBMED: 17088140$]$

Gevaert P, Van Zele T, Stammberger $\mathrm{H}$, Staudinger $\mathrm{H}$, Tavernier J, van Cauwenberge P, et al. Nasal interleukin-5 levels determine the response to anti-interleukin- 5 treatment in nasal polyp patients. 3rd EAACI Davos Meeting in Basic Immunology in Allergy and Clinical Immunology. Davos, Switzerland, 3-6 February, 2005. 2005. [CENTRAL: CN-00519575; CRS: 1362295]

Gevaert P, can Zele T, Stammberger H, Sacks H, van Cauwenberge, Bachert C. Anti-interleukin-5 treatment in nasal polyposis. Journal of Allergy and Clinical Immunology 2005;115(2):S138. [DOI: 10.1016/j.jaci.2004.12.566]

\section{Gevaert 2008 \{published data only\}}

Gevaert P, Van Bruaene N, Blomme K, Sousa AR, Marshal RP, Bachert C. Mepolizumab, a humanised anti-IL-5 monoclonal antibody, as treatment of severe nasal polyposis. American Academy of Allergy, Asthma and Immunology (AAAAI) 64th Annual Meeting. Philadelphia, PA, USA, March 14-18, 2008. Journal of Allergy and Clinical Immunology 2008;121(2 (Suppl 1)):Abstract No. L26. [CRS: 1449627]

\section{Gonzalez-Diaz 2014 \{published data only\}}

Gonzalez-Diaz SN, Rangel-Garza L. Omalizumab efficiency in patients with allergic rhinitis and chronic sinusitis. World Allergy Organization Journal 2014;7(Suppl 1):9. [CRS: 12486358]

\section{Hellings 2017 \{published data only\}}

Hellings P, Bachert C, Mullol J, Hamilos D, Naclerio R, Joish V, et al. Dupilumab improves all ACQ-5 individual items in patients with chronic rhinosinusitis with nasal polyposis (CRSwNP) and asthma: results from a phase 2a trial. European Respiratory Journal 2017;50(Suppl 61):PA3549. [CRS: 9863239]

Hellings P, Bachert C, Mullol J, Hamilos D, Naclerio R, Mannent $L$, et al. Dupilumab improves ACQ-5 items in CRSwNP patients with comorbid asthma. Respiratology 2018;23(Suppl 1):153. [DOI: 10.1111/resp.13268]

\section{Laidlaw 2019 \{published data only\}}

Laidlaw TM, Mullol J, Fan C, Zhang D, Amin N, Khan A, et al. Dupilumab improves nasal polyp burden and asthma control in patients with CRSwNP and AERD. Journal of Allergy and Clinical Immunology: In Practice 2019;7(7):2462-5.e1. [CRS: 12486356]

\section{Liberty Asthma Quest \{published data only\}}

Bousquet J, Maspero JF, Chipps BE, Corren J, FitzGerald JM, Chen Z, et al. Dupilumab consistently improves rhinoconjunctivitis-specific health-related quality of life in patients with uncontrolled, moderate-to-severe asthma and comorbid allergic rhinitis: results from the phase 3 LIBERTY ASTHMA QUEST study. Journal of Allergy and Clinical Immunology 2019;143(2 Suppl):AB101. [CENTRAL: CN-01932317; CRS: 9965843; EMBASE: 2001510554]

Busse W, Maspero JF, Katelaris CH, Saralaya D, Guillonneau S, Zhang B, et al. Dupilumab improves SNOT-22 scores in asthma patients with chronic rhinosinusitis or nasal polyposis (CRS/NP) in liberty asthma quest. European Respiratory Journal 2018;52(Suppl 62):PA1125. [CRS: 10534564; DOI: 10.1183/13993003.congress-2018.PA1125]

Busse WW, Maspero JF, Hanania NA, FitzGerald JM, Ford LB, Rice $M$, et al. Dupilumab improves lung function and reduces severe exacerbation rate in patients with uncontrolled, moderate-to-severe asthma with or without comorbid allergic rhinitis: results from the phase 3 LIBERTY ASTHMA QUEST study. Journal of Allergy and Clinical Immunology 2019;143(2 Suppl):AB97. [CENTRAL: CN-01945651; CRS: 10053593; EMBASE: 2001510335]

Busse WW, Maspero JF, Rabe KF, Papi A, Wenzel SE, Ford LB, et al. Liberty asthma QUEST: phase 3 randomized, double-blind, placebo-controlled, parallel-group study to evaluate dupilumab efficacy/safety in patients with uncontrolled, moderate-tosevere asthma. Advances in Therapy 2018;35(5):1-12. [CENTRAL: CN-01612071; CRS: 8435206; EMBASE: 622013044; PUBMED: 29725983]

Castro M, Busse WW, Zhang B, Maroni J, Rowe P, Amin N, et al. Dupilumab treatment produces rapid and sustained improvements in FEV1 in patients with uncontrolled, moderateto-severe asthma from the LIBERTY ASTHMA QUEST study. American Journal of Respiratory and Critical Care Medicine 2018;197:A6163. [CENTRAL: CN-01619354; CRS: 8919994; EMBASE: 622964392]

Castro M, Corren J, Hanania N, Pavord I, Quirce S, Thangavelu K, et al. Dupilumab efficacy in uncontrolled, moderate-tosevere allergic asthma in the phase 3 liberty asthma quest study. Annals of Allergy, Asthma and Immunology 2018;121 (5 Suppl):S8. [CENTRAL: CN-01680382; CRS: 9723247; EMBASE: 2001294249]

Castro M, Corren J, Pavord I D, Maspero J F, Wenzel S E, Rabe K F, et al. A randomized, controlled phase 3 study, LIBERTY ASTHMA QUEST, evaluating the efficacy and safety of dupilumab in uncontrolled moderate-to-severe asthma. American Journal of Respiratory and Critical Care Medicine 2018;197:A7700. [CENTRAL: CN-01619139; CRS: 8919610; EMBASE: 622968870]

Castro M, Corren J, Pavord ID, Maspero J, Wenzel S, Rabe KF, et al. Dupilumab efficacy and safety in moderate-to-severe uncontrolled asthma. New England Journal of Medicine 2018;378(26):2486-96. [CENTRAL: CN-01614085; CRS: 8574637; PUBMED: 29782217

Castro M, Msapero JF, Staudinger H, Jayawardena S, Maroni J, Rowe $P$, et al. Dupilumab improves lung function and reduces severe exacerbations in uncontrolled persistent asthma 
patients with high and low reversibility. European Respiratory Journal 2017;50(Suppl 61):PA4081. [CRS: 11161230; DOI: 10.1183/1393003.congress-2017.PA4018]

Corren J, Bousquet J, Busse WW, Maspero JF, Hanania NA, Ford LB, et al. Dupilumab suppresses inflammatory biomarkers in asthma patients with or without allergic rhinitis: post hoc analysis of the LIBERTY ASTHMA QUEST study. Journal of Allergy and Clinical Immunology 2019;143(2 Suppl):AB97. [CENTRAL: CN-01932588; CRS: 10053613; EMBASE: 2001509971]

Corren J, Castro M, Chanez P, Fabbri L, Joish VN, Amin N, et al. Dupilumab improves symptoms, quality of life, and productivity in uncontrolled persistent asthma. Annals of Allergy, Asthma \& Immunology 2019;122(1):41-9.e2. [CENTRAL: CN-01680383; CRS: 9508016; EMBASE: 2001274049; PUBMED: 30138668]

Corren J, Castro M, Guillonneau S, Chao J, Amin N, Pirozzi G, et al. Dupilumab produces rapid and sustained improvements in asthma-related symptoms in patients with uncontrolled, moderate-to-severe asthma from the LIBERTY ASTHMA QUEST study. American Journal of Respiratory and Critical Care Medicine 2018;197:A5948. [CENTRAL: CN-01619259; CRS: 8919832; EMBASE: 622966675]

Corren J, Castro M, Jayawardena S, Joish V, Amin N, Pirozzi G, et al. Dupilumab improves asthma control and asthma-related quality of life in uncontrolled persistent asthma patients across all baseline exacerbation rates. Chest 2017;152 (4 Suppl 1):A26. [CENTRAL: CN-01428595; CRS: 7210894; EMBASE: 619297553]

Corren J, Castro M, Maspero J, Cosio B, Kuna P, Chen Z, et al. Dupilumab improves asthma control in patients with uncontrolled, moderate-to-severe asthma, regardless of exacerbation history. Annals of Allergy, Asthma and Immunology 2018;121(5 Suppl):S42-3. [CENTRAL: CN-01680384; CRS: 9723268; EMBASE: 2001294081]

Corren J, Castro M, Maspero JF, Santiago ALV, Kuna P, Guillonneau S, et al. Dupilumab improves asthma-related patient reported outcomes in asthma patients with chronic rhinosinusitis or nasal polyposis (CRS/NP) in liberty asthma quest. European Respiratory Journal 2018;52(Suppl 62):PA1124. [CRS: 10534574; DOI: 10.1183/13993003.congress-2018.PA1124]

Fabbri LM, Bernstein JA, Staudinger H, Maroni J, Rowe P, Jayawardena $S$, et al. Dupilumab efficacy in severe asthma exacerbations by different baseline patient characteristics in patients with uncontrolled persistent asthma. Allergy 2017;72:108-9. [CENTRAL: CN-01417608; CRS: 6780311; EMBASE: 618250297]

Katelaris C, Rabe K, Corren J, Langton D, Bardin P, Park H, et al. Dupilumab improves asthma outcomes regardless of baseline lung function. Respirology (Carlton, Vic.) 2019;24(Suppl 1):110. [CENTRAL: CN-01946148; CRS: 10649712; EMBASE: 626940558]

Katelaris CH, Maspero JF, Jayawardena S, Rowe P, Maroni J, Pirozzi G, et al. Dupilumab efficacy and effect on asthma control in patients with uncontrolled persistent asthma and comorbid chronic rhinosinusitis with or without nasal polyps. Internal Medicine Journal 2017;47(Suppl 5):22. [CENTRAL: CN-01622495; CRS: 6943429; EMBASE: 618562783]
Katial R, Joish VN, Amin N, Rowe P, Maroni J, Pirozzi G, et al. Dupilumab improves patient-reported outcomes in uncontrolled persistent asthma patients with ongoing allergic rhinitis. European Respiratory Journal 2017;50(Suppl 61):PA3351. [CRS: 9863228; DOI: 10.1183/1393003.congress-2017.PA3551]

Korn S, Corren J, Castro M, Maspero J, Chen Z, Niemann I, et al. Dupilumab improved asthma control in patients with uncontrolled, moderate-to-severe asthma, regardless of exacerbations in the previous year. Pneumologie (Stuttgart, Germany) 2019;73(Suppl 1):P04. [CRS: 11766873; DOI: 10.1055/ s-0039-1678039]

Maspero J, Busse WW, Katelaris CH, Yanez A, Guillonneau S, Chen Z, et al. Dupilumab improves health related quality of life in uncontrolled, moderate-to-severe asthma patients with comorbid allergic rhinitis from the phase 3 LIBERTY ASTHMA QUEST study. Allergy. Netherlands: Blackwell Publishing Ltd, 2018; Vol. 73, issue Suppl 105:30. [CENTRAL: CN-01655010; CRS: 9254487; EMBASE: 623867942]

Maspero JF, Corren J, Ford LB, Sher L, Chipps BE, Peters AT, et al. Dupilumab suppresses type 2 biomarkers in asthma patients with and without comorbid chronic rhinosinusitis with or without nasal polyposis (CRS/NP): post hoc analysis of LIBERTY ASTHMA QUEST. Journal of Allergy and Clinical Immunology 2019;143(2 Suppl):AB98. [CENTRAL: CN-01945714; CRS: 10053578; EMBASE: 2001510892]

Maspero JF, Katelaris C, Jayawardena S, Rowe P, Maroni J, Pirozzi G, et al. Dupilumab efficacy in uncontrolled persistent asthma patients with history of comorbid chronic rhinosinusitis with or without nasal polyps. American Journal of Respiratory and Critical Care Medicine. Netherlands: American Thoracic Society, 2017; Vol. 195. [CENTRAL: CN-01408935; CRS: 6624660; EMBASE: 617708346]

NCT02414854. Evaluation of dupilumab in patients with persistent asthma (liberty asthma quest) [A randomized, double blind, placebo-controlled, parallel group study to evaluate the efficacy and safety of dupilumab in patients with persistent asthma]. https://clinicaltrials.gov/show/nct02414854 (first received 13 April 2015). [CENTRAL: CN-01505676; CRS: 7497877]

Pavord I, Ford LB, Corren J, Kuna P, Dong Q, Staudinger H, et al. Dupilumab reduces exacerbations and improves lung function in uncontrolled, moderate-to-severe asthma patients regardless of prior exacerbation history in the phase 3 liberty asthma quest study. Thorax 2018;73:A121-2. [CRS: 12084387]

Pavord I, Papi A, Wenzel S, Park H, Zhang B, Staudinger H, et al. Dupilumab reduces risk of severe exacerbations and improves FEV1 in patients on both high-and medium dose ICS with uncontrolled, moderate-to-severe asthma from the phase 3 LIBERTY ASTHMA QUEST Study. Allergy 2018;73(Suppl 105):463-4. [CENTRAL: CN-01655016; CRS: 9254514; EMBASE: $623867426]$

Pavord ID, Ford L, Sher L, Rabe KF, Park H-S, Cosio BG, et al. Dupilumab efficacy in asthma patients with comorbid chronic rhinosinusitis or nasal polyposis (CRS/ NP) in LIBERTY ASTHMA QUEST. European Respiratory 
Journal 2018;52(Suppl 62):OA1651. [CRS: 10534548; DOI: 10.1183/13993003.congress-2018.0A1651]

Weinstein S, Staudinger H, Guillonneau S, Taniou C, Eckert L, Maroni J, et al. Dupilumab improves FEV $<$ inf $>1</$ inf $>$ and exacerbations in asthma with allergic rhinitis. Respirology (Carlton, Vic.). Netherlands: Blackwell Publishing, 2018; Vol. 23, issue Suppl 1:154. [CENTRAL: CN-01911329; CRS: 8435106; EMBASE: 622091654]

Weinstein SF, Katial R, Jayawardena S, Pirozzi G, Staudinger H, Eckert $L$, et al. Dupilumab improves sinonasal symptoms of perennial allergic rhinitis (PAR) in uncontrolled persistent asthma patients with comorbid PAR. Allergy and Asthma Proceedings 2017;38(3):237. [CENTRAL: CN-01477100; CRS: 7611958; EMBASE: 620614985]

Weinstein SF, Katial R, Jayawardena S, Pirozzi G, Staudinger H, Eckert $L$, et al. Efficacy and safety of dupilumab in perennial allergic rhinitis and comorbid asthma. Journal of Allergy and Clinical Immunology 2018;142(1):171-7.e1. [CENTRAL: CN-01643878; CRS: 7517279; EMBASE: 620773805; PUBMED: 29355679]

Wenzel S, Pavord ID, Rabe KF, Papi A, Mark Fitzgerald J, Jagerschmidt A, et al. Dupilumab shows rapid and sustained suppression of inflammatory biomarkers in asthma patients in liberty asthma quest. European Respiratory Journal 2018;52(Suppl 62):PA5005. [CENTRAL: CN-01967194; CRS: 11120829; EMBASE: 626625174]

Zhang L, Li M, Meng Z, Davis JD, Kanamaluru V, Lu Q. Semimechanistic pharmacokinetic/pharmacodynamic (PK/PD) modeling of dupilumab on pre-bronchodilator forced expiratory volume in 1 second (FEV1) in uncontrolled moderate-to-severe asthma. Journal of Pharmacokinetics and Pharmacodynamics 2018;45(Suppl 1):S69-70. [CENTRAL: CN-01924653; CRS: 9294772; EMBASE: 624153193]

\section{MUSCA \{published data only\}}

NCT02281318. Efficacy and safety study of mepolizumab adjunctive therapy in participants with severe eosinophilic asthma on markers of asthma control [A randomised, doubleblind, placebo-controlled, parallel-group, multi-centre 24week study to evaluate the efficacy and safety of mepolizumab adjunctive therapy in subjects with severe eosinophilic asthma on markers of asthma control]. https://clinicaltrials.gov/show/ nct02281318 (first received 3 November 2014). [CRS: 3545753]

Nelsen L, Bradford ES, Bratton DJ, Albers FC, Brusselle G. Improvement in rhinosinusitis health related quality of life in patients with severe eosinophilic asthma. European Respiratory Journal 2017;50(Suppl 61):PA3583. [CRS: 10403903; DOI: 10.1183/1393003.congress-2017.PA3583]

\section{Naclerio 2017 \{published data only\}}

Naclerio RM, Hamilos DL, Ferguson BJ, Bachert C, Hellings PW, Mullol J, et al. Dupilumab improves sense of smell and reduces anosmia among patients with nasal polyposis and chronic sinusitis: results from a phase 2a trial. Journal of Allergy and Clinical Immunology 2017;139(2 Suppl):AB90. [CRS: 8071505]

\section{NCT00603785 \{published data only\}}

NCT00603785. Effects of anti-IgE antibody omalizumab on patients with chronic sinusitis [Effects of anti-IgE antibody omalizumab (Xolair) on patients with chronic sinusitis and a positive allergen test]. https://clinicaltrials.gov/show/ nct00603785 (first received 29 January 2008). [CRS: 1643067]

\section{NCT01285323 \{published data only\}}

Castro M, Zangrilli J, Wechsler ME, Bateman ED, Brusselle GG, Bardin P, et al. Reslizumab for inadequately controlled asthma with elevated blood eosinophil counts: results from two multicentre, parallel, double-blind, randomised, placebocontrolled, phase 3 trials. Lancet Respiratory Medicine 2015;3(5):355-66. [CRS: 1797555; PUBMED: 25736990]

NCT01285323. A study to evaluate the efficacy and safety of reslizumab in patients with eosinophilic asthma [A 12month, double-blind, placebo-controlled, parallel-group study to evaluate the efficacy and safety of reslizumab (3.0 $\mathrm{mg} / \mathrm{kg}$ ) in the reduction of clinical asthma exacerbations in patients (12-75 years of age) with eosinophilic asthma]. https:// clinicaltrials.gov/show/NCT01285323 (first received 28 January 2011). [CRS: 6716614]

Weinstein SF, Germinaro M, Bardin P, Korn S, Bateman ED. Efficacy of reslizumab with asthma, chronic sinusitis with nasal polyps and elevated blood eosinophils. Journal of Allergy and Clinical Immunology 2016;137(2 Suppl 1):AB86. [CRS: 1865798]

Weinstein SF, Katial RK, Bardin P, Korn S, McDonald M, Garin M, et al. Effects of reslizumab on asthma outcomes in a subgroup of eosinophilic asthma patients with self-reported chronic rhinosinusitis with nasal polyps. Journal of Allergy \& Clinical Immunology in Practice 2019;7(2):589-96.e3. [CENTRAL: CN-01922720; CRS: 9618835; EMBASE: 2001152799; PUBMED: 30193936]

\section{NCT02170337 \{published data only\}}

NCT02170337. A study to evaluate the safety, tolerability, pharmacokinetics, and pharmacodynamics of AMG 282 in healthy subjects and subjects with chronic rhinosinusitis with nasal polyps [A randomized, double-blind, placebocontrolled, ascending multiple-dose study to evaluate the safety, tolerability, pharmacokinetics, and pharmacodynamics of AMG 282 in healthy subjects and subjects with chronic rhinosinusitis with nasal polyps]. https://clinicaltrials.gov/ show/nct02170337 (first received 19 June 2014). [CRS: 8298441]

\section{NCT02734849 \{published data only\}}

NCT02734849. Study to evaluate multiple doses in patients with nasal polyposis [A phase 2, randomized, double-blind, placebo-controlled, study to evaluate multiple doses of AK001 in patients with moderate to severe nasal polyposis]. https:// clinicaltrials.gov/show/NCT02734849 (first received 12 April 2016). [CENTRAL: CN-01415083; CRS: 6977025]

\section{NCT02743871 \{published data only\}}

NCT02743871. Study of PF-06817024 in healthy subjects, in patients with chronic rhinosinusitis with nasal polyps and in patients with atopic dermatitis [A phase 1 , randomized, doubleblind, third-party open, placebo-controlled, dose escalating study to evaluate the safety, tolerability, pharmacokinetics 
and pharmacodynamics of single and/or multiple intravenous and/or subcutaneous doses of pf-06817024 in healthy subjects who may BE mildly atopic, subjects with chronic rhinosinusitis with nasal polyps, and subjects with moderate-severe atopic dermatitis]. https://clinicaltrials.gov/show/NCT02743871 2016. [CRS: 6978629]

\section{Perez De Llano 2018 \{published data only\}}

Perez De Llano L, Meizlik P, McDonald M, Mustafa SS. Reslizumab decreases nasal adverse events and upper respiratory-associated concomitant medication use in patients with eosinophilic asthma and nasal polyps. Allergy 2018;73(Suppl 105):91-2. [CRS: 12486354]

\section{Tajiri 2013 \{published data only\}}

Tajiri T, Matsumoto H, Hiraumi H, Ikeda H, Morita K, Izuhara K, et al. Efficacy of omalizumab in eosinophilic chronic rhinosinusitis patients with asthma. Annals of Allergy, Asthma, \& Immunology 2013;110(5):387-8. [CRS: 12486360]

\section{Zangrilli 2019 \{published data only\}}

Zangrilli JG, Maspero J, Harrison T, Werkstrom V, Wu Y. Clinical efficacy of benralizumab in patients with severe, uncontrolled eosinophilic asthma and nasal polyposis: pooled Analysis of the SIROCCO and CALIMA Trials. Pneumologie (Stuttgart, Germany) 2019;73(Suppl 1):AB12. [CRS: 11766888; DOI: 10.1016/ j.jaci.2017.12.038]

\section{References to ongoing studies}

\section{NCT02772419 \{published data only\}}

NCT02772419. Study of benralizumab (KHK4563) in patients with eosinophilic chronic rhinosinusitis [A phase 2, doubleblind, placebo-controlled study of benralizumab (KHK4563) in patients with eosinophilic chronic rhinosinusitis]. https:// clinicaltrials.gov/show/NCT02772419 (first received 13 May 2016). [CENTRAL: CN-01415166; CRS: 6978699]

\section{NCT02799446 \{published data only\}}

NCT02799446. Effect of reslizumab in chronic rhinosinusitis [Efficacy of reslizumab for the treatment of chronic rhinosinusitis a double blind, randomized, placebo-controlled, phase III trial]. https://clinicaltrials.gov/show/NCT02799446 (first received 14 June 2016). [CRS: 6978626]

\section{NCT03450083 \{published data only\}}

NCT03450083. Benralizumab effect on severe chronic rhinosinusitis with eosinophilic polyposis [Benralizumab effect on severe chronic rhinosinusitis with eosinophilic polyposis: a phase II randomized placebo controlled trial]. https:// clinicaltrials.gov/show/nct03450083 (first received 17 August 2017). [CRS: 8239610]

\section{NCT03614923 \{published data only\}}

NCT03614923. Etokimab in adult patients with chronic rhinosinusitis with nasal polyps (CRSwNP) [A phase 2 doubleblind, placebo-controlled multi-dose study to investigate etokimab (ANB020) activity in adult patients with chronic rhinosinusitis with nasal polyps]. https://clinicaltrials.gov/ show/NCT03614923 (first received 3 August 2018). [CRS: 9157986]

\section{OSTRO \{published data only\}}

NCT03401229. Efficacy and safety study of benralizumab for patients with severe nasal polyposis [A multicenter, randomized, double-blind, parallel-group, placebo-controlled phase 3 efficacy and safety study of benralizumab in patients with severe nasal polyposis]. https://clinicaltrials.gov/show/ NCT03401229 (first received 17 January 2018). [CRS: 8275488]

\section{POLYP 1 \{unpublished data only\}}

NCT03280550. A clinical trial of omalizumab in participants with chronic rhinosinusitis with nasal polyps [A phase III, randomized, multicenter, double-blind, placebo-controlled clinical trial of omalizumab in patients with chronic rhinosinusitis with nasal polyps]. https://clinicaltrials.gov/ show/NCT03280550 (first received 12 September 2017). [CENTRAL: CN-01415214; CRS: 6978602]

\section{POLYP 2 \{unpublished data only\}}

NCT03280537. A clinical trial of omalizumab in participants with chronic rhinosinusitis with nasal polyps [A phase III, randomized, multicenter, double-blind, placebo-controlled clinical trial of omalizumab in patients with chronic rhinosinusitis with nasal polyps]. https://clinicaltrials.gov/ show/NCT03280537 (first received 12 September 2017). [CENTRAL: CN-01415096; CRS: 6977504]

\section{SYNAPSE \{published data only\}}

EUCTR2016-004255-70-SE. Effect of mepolizumab in severe bilateral nasal polyps [A randomised, double-blind, parallel group PhIll study to assess the clinical efficacy and safety of 100 mg SC mepolizumab as an add on to maintenance treatment in adults with severe bilateral nasal polyps - SYNAPSE (StudY in NAsal Polyps patients to assess the Safety and Efficacy of mepolizumab)]. https://www.clinicaltrialsregister.eu/ctr-search/ trial/2016-004255-70/SE (first received 4 October 2017). [CRS: 10787349]

NCT03085797. Effect of mepolizumab in severe bilateral nasal polyps [A randomised, double-blind, parallel group PhIII study to assess the clinical efficacy and safety of $100 \mathrm{mg}$ SC mepolizumab AS an add on to maintenance treatment in adults with severe bilateral nasal polyps - SYNAPSE (study in nasal polyps patients to assess the safety and efficacy of mepolizumab)]. https://clinicaltrials.gov/show/NCT03085797 (first received 21 March 2017). [CRS: 6978655]

\section{Additional references}

\section{Cho 2012}

Cho SH, Hong SJ, Han B, Lee SH, Suh L, Norton J, et al. Age-related differences in the pathogenesis of chronic rhinosinusitis. Journal of Allergy and Clinical Immunology 2012;129(3):858-60.e2.

\section{Chong 2016a}

Chong LY, Head K, Hopkins C, Philpott C, Schilder AGM, Burton MJ. Intranasal steroids versus placebo or no intervention for chronic rhinosinusitis. Cochrane Database of Systematic Reviews 2016, Issue 4. [DOI: 10.1002/14651858.CD011996.pub2] 


\section{Chong 2016b}

Chong LY, Head K, Hopkins C, Philpott C, Glew S, Scadding G, et al. Saline irrigation for chronic rhinosinusitis. Cochrane Database of Systematic Reviews 2016, Issue 4. [DOI: 10.1002/14651858.CD011995.pub2]

\section{Chong 2016c}

Chong LY, Head K, Hopkins C, Philpott C, Burton MJ, Schilder AGM. Different types of intranasal steroids for chronic rhinosinusitis. Cochrane Database of Systematic Reviews 2016 , Issue 4. [DOI: 10.1002/14651858.CD011993.pub2]

\section{DeMarcantonio 2011}

DeMarcantonio MA, Han JK. Nasal polyps: pathogenesis and treatment implications. Otolaryngologic Clinics of North America 2011;44(3):685-95, ix.

\section{Ebbens 2010}

Ebbens FA, Toppila-Salmi SK, Renkonen JA, Renkonen RL, Mullol J, van Drunen CM, et al. Endothelial L-selectin ligand expression in nasal polyps. Allergy 2010;65(1):95-102.

\section{Ebbens 2011}

Ebbens FA, Toppila-Salmi S, de Groot EJ, Renkonen J, Renkonen R, van Drunen CM, et al. Predictors of post-operative response to treatment: a double blind placebo controlled study in chronic rhinosinusitis patients. Rhinology 2011;49(4):413-9.

\section{Egger 1997}

Egger M, Davey Smith G, Schneider M, Minder C. Bias in metaanalysis detected by a simple, graphical test. BMJ (Clinical research ed.) 1997;315(7109):629-34.

\section{EPOS 2007}

Fokkens W, Lund V, Mullol J, European Position Paper on Rhinosinusitis and Nasal Polyps Group. European position paper on rhinosinusitis and nasal polyps 2007. Rhinology. Supplement 2007;45 Suppl 20:1-136.

\section{EPOS 2012}

Fokkens WJ, Lund VJ, Mullol J, Bachert C, Alobid I, Baroody F, et al. European Position Paper on Rhinosinusitis and Nasal Polyps 2012. Rhinology. Supplement 2012;50 Suppl 23:1-298.

\section{FDA 2018}

US Food, Drug Administration. CFR - Code of Federal Regulations Title 21. https://www.accessdata.fda.gov/scripts/ cdrh/cfdocs/cfcfr/cfrsearch.cfm?fr=312.32 (accessed 18 February 2019).

\section{Gliklich 1995}

Gliklich RE, Metson R. The health impact of chronic sinusitis in patients seeking otolaryngologic care. Otolaryngology - Head and Neck Surgery 1995;113(1):104-9.

\section{Handbook 2011}

Higgins JPT, Green S (editors). Cochrane Handbook for Systematic Reviews of Interventions Version 5.1.0 [updated March 2011]. The Cochrane Collaboration, 2011. Available from www.cochrane-handbook.org.

\section{Handbook 2019}

Higgins JPT, Thomas J, Chandler J, Cumpston M, Li T, Page MJ, Welch VA (editors). Cochrane Handbook for Systematic Reviews of Interventions version 6.0 (updated July 2019). Cochrane, 2019. Available from www.training.cochrane.org/handbook.

\section{Hastan 2011}

Hastan D, Fokkens WJ, Bachert C, Newson RB, Bislimovska J, Bockelbrink $A$, et al. Chronic rhinosinusitis in Europe - an underestimated disease. A GA2LEN study. Allergy 2011;66(9):1216-23.

\section{Head 2016a}

Head K, Chong LY, Hopkins C, Philpott C, Burton MJ, Schilder AGM. Short-course oral steroids alone for chronic rhinosinusitis. Cochrane Database of Systematic Reviews 2016, Issue 4. [DOI: 10.1002/14651858.CD011991.pub2]

\section{Head 2016b}

Head K, Chong LY, Hopkins C, Philpott C, Schilder AGM, Burton MJ. Short-course oral steroids as an adjunct therapy for chronic rhinosinusitis. Cochrane Database of Systematic Reviews 2016, Issue 4. [DOI: 10.1002/14651858.CD011992.pub2]

\section{Head 2016c}

Head K, Chong LY, Piromchai P, Hopkins C, Philpott C, Schilder AGM, et al. Systemic and topical antibiotics for chronic rhinosinusitis. Cochrane Database of Systematic Reviews 2016, Issue 4. [DOI: 10.1002/14651858.CD011994.pub2]

\section{Head 2018}

Head K, Sacks PL, Chong LY, Hopkins C, Philpott C. Topical and systemic antifungal therapy for chronic rhinosinusitis. Cochrane Database of Systematic Reviews 2018, Issue 9. [DOI: 10.1002/14651858.CD012453.pub2]

\section{Hoehle 2019}

Hoehle LP, Phillips KM, Speth MM, Caradonna DS, Gray ST, Sedaghat AR. Responsiveness and minimal clinically important difference for the EQ-5D in chronic rhinosinusitis. Rhinology 2019;57(2):110-6.

\section{Hong 2015}

Hong CJ, Tsang AC, Quinn JG, Bonaparte JP, Stevens A, Kilty SJ. Anti-IgE monoclonal antibody therapy for the treatment of chronic rhinosinusitis: a systematic review. Systematic Reviews 2015;4:166. [DOI: 10.1186/s13643-015-0157-5]

\section{Hopkins 2009}

Hopkins C, Gillett S, Slack R, Lund VJ, Browne JP. Psychometric validity of the 22-item Sinonasal Outcome Test. Clinical Otolaryngology 2009;34(5):447-54. [DOI: 10.1111/ j.1749-4486.2009.01995.x]

\section{Hopkins 2018}

Hopkins C, Hettige R, Soni-Jaiswal A, Lakhani R, Carrie S, et al. CHronic Rhinosinusitis Outcome MEasures (CHROME), developing a core outcome set for trials of interventions in chronic rhinosinusitis. Rhinology 2018;56(1):22-32. [PUBMED: 29306959] 


\section{Jefferson 2018}

Jefferson T, Doshi P, Boutron I, Golder S, Heneghan C, Hodkinson A, et al. When to include clinical study reports and regulatory documents in systematic reviews. $B M J$ Evidence-Based Medicine 2018;23(6):210-7. [DOI: 10.1136/ bmjebm-2018-110963]

\section{Kariyawasam 2019}

Kariyawasam HH. Chronic rhinosinusitis with nasal polyps: insights into mechanisms of disease from emerging biological therapies. Expert Review of Clinical Immunology 2019;15(1):59-71. [DOI: 10.1080/1744666X.2019.1541738]

\section{Kern 2008}

Kern RC, Conley DB, Walsh W, Chandra R, Kato A, TripathiPeters $A$, et al. Perspectives on the etiology of chronic rhinosinusitis: an immune barrier hypothesis. American Journal of Rhinology 2008;22(6):549-59.

\section{Keswani 2012}

Keswani A, Chustz RT, Suh L, Carter R, Peters AT, Tan BK, et al. Differential expression of interleukin-32 in chronic rhinosinusitis with and without nasal polyps. Allergy 2012;67(1):25-32.

\section{Larsen 2004}

Larsen P, Tos M. Origin of nasal polyps: an endoscopic autopsy study. Laryngoscope 2004;114(4):710-9.

\section{Marshal 2018}

Marshall J, Noel-Storr AH, Kuiper J, Thomas J, Wallace BC. Machine learning for identifying randomized controlled trials: an evaluation and practitioner's guide. Research Synthesis Methods 2018;9(4):602-14.

\section{McDonald 2017}

McDonald S, Noel-Storr AH, Thomas J. Harnessing the efficiencies of machine learning and Cochrane Crowd to identify randomised trials for individual Cochrane reviews. Global Evidence Summit; 2017 Sep 13-17; Cape Town, South Africa. 2017.

\section{NICE 2019}

National Institute for Health and Care Excellence (NICE). Dupilumab for treating chronic rhinosinusitis with nasal polyps ID1179. https://www.nice.org.uk/guidance/proposed/gidta10450 (accessed 18 February 2019).

\section{Noel-Storr 2018}

Noel-Storr AH, The Project Transform Team. Cochrane Crowd: new ways of working together to produce health evidence. Evidence Live; 2018 Jun 18-20; Oxford, UK. 2018.

\section{Philpott 2015}

Philpott C, Hopkins C, Erskine S, Kumar N, Robertson A, et al. The burden of revision sinonasal surgery in the UK-data from the Chronic Rhinosinusitis Epidemiology Study (CRES): a cross-sectional study. BMJ Open 2015;5:e006680. [DOI: 10.1136/ bmjopen-2014-006680]

\section{Ragab 2004}

Ragab SM, Lund VJ, Scadding G. Evaluation of the medical and surgical treatment of chronic rhinosinusitis: a prospective, randomised, controlled trial. Laryngoscope 2004;114(5):923-30.

\section{Ragab 2010}

Ragab SM, Lund VJ, Scadding G, Saleh HA, Khalifa MA. Impact of chronic rhinosinusitis therapy on quality of life: a prospective randomized controlled trial. Rhinology 2010;48(3):305-11.

\section{RevMan Web 2019 [Computer program]}

The Cochrane Collaboration. Review Manager Web (RevMan Web). The Cochrane Collaboration, 2019.

\section{Rivero 2017}

Rivero A, Liang J. Anti-IgE and anti-IL5 biologic therapy in the treatment of nasal polyposis: a systematic review and meta-analysis. Annals of Otology, Rhinology, and Laryngology 2017;126(11):739-47. [DOI: 10.1177/0003489417731782]

\section{Simmonds 2017}

Simmonds M, Salanti G, McKenzie J, Elliott J, Living Systematic Review Network. Living systematic reviews: 3. Statistical methods for updating meta-analyses. Journal of Clinical Epidemiology 2017;91:38-46. [DOI: 10.1016/ j.jclinepi.2017.08.008]

\section{Smith 2018}

Smith KA, Pulsipher A, Gabrielsen DA, Alt JA. Biologics in chronic rhinosinusitis: an update and thoughts for future directions. American Journal of Rhinology \& Allergy 2018;32(5):412-23. [DOI: 10.1177/1945892418787132]

\section{Smith 2019}

Smith KA, Orlandi RR, Oakley G, Meeks H, Curtin K, Alt JA. Longterm revision rates for endoscopic sinus surgery. International Forum of Allergy \& Rhinology 2019 2019;9(4):402-8.

\section{Sterne 2019}

Sterne JAC, Savovic J, Page MJ, Elbers RG, Blencowe NS, Boutron I, et al. RoB 2: a revised tool for assessing risk of bias in randomised trials. BMJ (Clinical research ed.) 2019;366:14898. [DOI: 10.1136/bmj.l4898; PUBMED: 31462531]

\section{Tan 2011}

Tan BK, Li QZ, Suh L, Kato A, Conley DB, Chandra RK, et al. Evidence for intranasal antinuclear autoantibodies in patients with chronic rhinosinusitis with nasal polyps. Journal of Allergy and Clinical Immunology 2011;128(6):1198-206.e1.

\section{Thomas 2017}

Thomas J, Noel-Storr AH, Marshall I, Wallace B, McDonald S, Mavergames C, et al. Living Systematic Review Network. Living systematic reviews 2: combining human and machine effort. Journal of Clinical Epidemiology 2017;91:31-7. [DOI: 10.1016/ j.jclinepi.2017.08.011]

\section{Tomassen 2011}

Tomassen P, Van Zele T, Zhang N, Perez-Novo C, Van Bruaene N, Gevaert P, et al. Pathophysiology of chronic rhinosinusitis. Proceedings of the American Thoracic Society 2011;8(1):115-20. 


\section{Tsetsos 2018}

Tsetsos N, Goudakos JK, Daskalakis D, Konstantinidis I, Markou K. Monoclonal antibodies for the treatment of chronic rhinosinusitis with nasal polyposis: a systematic review. Rhinology 2018;56(1):11-21. [DOI: 10.4193/Rhin17.156]

\section{van Drunen 2009}

van Drunen CM, Reinartz SM, Wigman J, Fokkens W. Inflammation in chronic rhinosinusitis and nasal polyposis. Immunology and Allergy Clinics of North America 2009;29(4):621-9.

\section{Wallace 2017}

Wallace BC, Noel-Storr AH, Marshall IJ, Cohen AM, Smalheiser NR, et al. Identifying reports of randomized controlled trials (RCTs) via a hybrid machine learning and crowdsourcing approach. Journal of the American Medical Informatics Association 2017;24(6):1165-8. [DOI: 10.1093/jamia/ ocx053]

\section{Zhang 2008}

Zhang N, Van Zele T, Perez-Novo C, Van Bruaene N, Holtappels G, DeRuyck N, et al. Different types of T-effector cells orchestrate mucosal inflammation in chronic sinus disease. Journal of Allergy and Clinical Immunology 2008;122(5):961-8.

\section{Zhang 2009}

Zhang XH, Lu X, Long XB, You XJ, Gao QX, Cui YH, et al. Chronic rhinosinusitis with and without nasal polyps is associated with decreased expression of glucocorticoid-induced leucine zipper. Clinical and Experimental Allergy 2009;39(5):647-54.

\section{References to other published versions of this review Chong 2019}

Chong LY, Piromchai P, Sharp S, Snidvongs K, Philpott C, Hopkins C, Burton MJ. Biologics for chronic rhinosinusitis. Cochrane Database of Systematic Reviews 2019, Issue 12. [DOI: 10.1002/14651858.CD013513]

* Indicates the major publication for the study

\section{CHARACTERISTICS OF STUDIES}

Characteristics of included studies [ordered by study ID]

Bachert 2016

Methods Double-blind, parallel-group RCT with 16 weeks of treatment/follow-up

Participants

Setting: multicentre; 13 hospitals/clinical centres in the USA and Europe (Belgium, Spain and Sweden)

Sample size: 60

- Number randomised: 60

- Number completed: 51 (28 in intervention group, 23 in comparator)

\section{Participant (baseline) characteristics}

- Age: mean 47.4 years dupilumab group; mean 49.3 years placebo group

- Gender: $60 \%$ male dupilumab group, $53.3 \%$ male placebo group

- Main diagnosis: chronic sinusitis with nasal polyps

- Polyps status: bilateral nasal polyp score (range 0 to 8, higher = worse) 5.9 (1.0) dupilumab group; 5.7 (0.9) placebo group

- Previous sinus surgery status: $53.3 \%$ had $\geq 1$ previous surgery for nasal polyps in dupilumab group; $63.3 \%$ of placebo group

- Previous courses of steroids: excluded if received oral corticosteroids within past 2 months

- Aspirin sensitivity: $20 \%$ of dupilumab group and $30 \%$ of placebo group

- Asthma: $53.3 \%$ dupilumab group and $63.3 \%$ placebo group

- Need for surgery: no information provided regarding whether participants were deemed to require surgery at baseline (no surgical outcomes reported)

\section{Inclusion criteria:}

- A minimum bilateral nasal polyp score of 5 out of a maximum score of 8 for both nostrils (with at least a score of 2 for each nostril) despite completion of a prior INCS treatment for at least 8 weeks before screening; and 
- Presence of at least 2 of the following symptoms prior to screening: nasal blockade/obstruction/congestion or nasal discharge (anterior/posterior nasal drip); facial pain/pressure; reduction or loss of smell.

The study had a prespecified enrolment goal that $50 \%$ of patients had comorbid asthma (based on patient history).

\section{Exclusion criteria:}

- Patients $<18$ or $>65$ years of age

- SNOT-22 score of $<7$

- Patients who have taken other investigational drugs or the following prohibited therapy within 2 months before screening or 5 half-lives, whichever is longer

* Burst of oral corticosteroids (OCS) or intranasal corticosteroid drops within the 2 months before screening or are scheduled to receive OCS during the study period for another condition

* Monoclonal antibody (mAb) and immunosuppressive treatment

* Anti-immunoglobulin E (IgE) therapy (omalizumab) within 130 days of Visit 1

* Leukotriene antagonists/modifiers unless patient is on a continuous treatment for at least 30 days prior to Visit 1

- Patients who have undergone nasal surgery within 6 months before screening or have had more than 2 surgeries in the past for nasal polyps

- Patients with conditions/concomitant diseases making them non-evaluable for the primary efficacy endpoint, such as:

* Antrochoanal polyps

* Nasal septal deviation that would occlude at least one nostril

* Acute sinusitis, nasal infection or upper respiratory infection at screening or in the 2 weeks before screening

* Ongoing rhinitis medicamentosa

* Churg-Strauss syndrome, Young's syndrome, Kartagener's syndrome or dyskinetic ciliary syndromes, concomitant cystic fibrosis

* Signs or a CT scan suggestive of Allergic fungal rhinosinusitis

- Patients with co-morbid asthma are excluded if one of the following criteria is met:

* Patients with $\mathrm{FEV}_{1}<60 \%$ (of predicted normal);

* Patients with an asthma exacerbation requiring systemic (oral and/or parenteral) steroid treatment or hospitalisation for $>24$ hours for treatment of asthma, within 3 months prior to screening or are on a dose of greater than $1000 \mu \mathrm{g}$ fluticasone or an equivalent inhaled corticosteroid.

Interventions

\section{Intervention $(\mathbf{n}=\mathbf{3 0})$ :}

- $600 \mathrm{mg}$ loading dose of subcutaneous dupilumab, followed by $300 \mathrm{mg}$ every week for 15 weeks

\section{Control $(\mathbf{n}=\mathbf{3 0})$ :}

- Placebo given subcutaneously every week for 16 weeks

Use of additional medication (common to both groups): $100 \mu \mathrm{g}$ mometasone furoate nasal spray in each nostril twice daily given during the 4-week run-in period and continued at a stable dose throughout the trial. Inhaled asthma controller therapies could be continued.

Outcomes

\section{Primary outcomes (relevant to this review):}

All reported at 16 weeks

- Disease specific health-related quality of life (SNOT-22 score)

- Disease severity symptom score (VAS score for "how troublesome are your symptoms?"; individual symptoms severity scores for nasal congestion/obstruction, anterior/posterior rhinorrhoea, loss of sense of smell, nocturnal awakenings)

- Severe adverse events 


\section{Secondary outcomes (relevant to this review):}

All reported at 16 weeks

- Endoscopic polyp score (change in bilateral score, range 0 to 8 , each nostril scored between 0 and 4; higher $=$ larger polyps)

- CT scan score (Lund Mackay CT score, range 0 to 24, higher = worse)

- Adverse events (nasopharyngitis)

\section{Other outcomes reported by the study:}

All reported at 16 weeks

- UPSIT smell test

- Peak nasal inspiratory flow

- Patient-rated nasal congestion/obstruction

- Anterior and posterior rhinorrhoea (score 0 to 3 )

- Loss of sense of smell (score 0 to 3)

- Nocturnal awakening (score 0 to 3 )

Funding sources Sanofi and Regeneron Pharmaceuticals

Declarations of interest Trial authors employed/received funding from Sanofi and Regeneron Pharmaceuticals. Sanofi and Regeneron Pharmaceuticals Inc, in collaboration with the academic clinical investigators, provided input on the design and conduct of the study; oversaw the collection, management and statistical analysis of data; and contributed to the interpretation of the data and the preparation, review and submission of the manuscript. The final decision on manuscript submission was made by the authors; the sponsors did not have the right to veto or require submission or publication.

Notes

A prespecified enrolment goal was that $50 \%$ of the patients had comorbid asthma. Recruitment of nasal polyps patients without co-morbid asthma would stop when approximately 28 patients without asthma were randomised.

Trial registration number NCT01920893.

\section{Risk of bias}

Bias Authors' judgement Support for judgement

Random sequence genera- Low risk tion (selection bias)
Quote: "A randomized treatment kit number list will be generated centrally by Sanofi. The investigational product (dupilumab or placebo) will be packaged in accordance with this list.

The Sanofi Clinical Supplies team will provide the randomized treatment kit number list and the Study Biostatistician will provide the randomization scheme to the centralized treatment allocation system. This centralized treatment allocation system will generate the patient randomization list according to which it will allocate the treatments to the patients."

Comment: central randomisation using computer software
Allocation concealment Low risk (selection bias)

\begin{abstract}
Quote: "This centralized treatment allocation system will generate the patient randomization list according to which it will allocate the treatments to the patients". "The Investigator obtains treatment kit numbers at randomization and subsequent scheduled visits via an Interactive Voice Response System/Interactive Web Response System (IVRS/IWRS) that will be available 24 hours a day." page 36 protocol
\end{abstract}

Comment: central allocation, separate to enrolment of participants 
Bachert 2016 (Continued)

Blinding of participants and personnel (performance bias)

All outcomes
Low risk Quote: "Dupilumab and placebo were provided in identical and indistinguishable treatment kits, and study patients, investigators, and site personnel were blinded to study treatment."

Comment: double-blind
Blinding of outcome as- Low risk sessment (detection bias)

All outcomes
Quote: "In accordance with the double-blind design, study patients, investigators, and study site personnel will remain blinded to study treatment and will not have access to the randomization (treatment codes)." "Video recordings of endoscopies were sent to an independent reviewer for centralized blinded data assessment."

Comment: blinded study

$\begin{array}{lll}\begin{array}{l}\text { Incomplete outcome data } \\ \text { (attrition bias) } \\ \text { All outcomes }\end{array} & \text { High risk } & \begin{array}{l}\text { Quote: "There were 23 patients in the placebo group who completed the 16- } \\ \text { week treatment period and } 28 \text { in the dupilumab group." }\end{array} \\ \begin{array}{l}\text { Comment: high dropout of } 7 / 30(23 \%) \text { in placebo arm versus } 2 / 30(7 \%) \text { in inter- } \\ \text { vention arm }\end{array} \\ \begin{array}{l}\text { Selective reporting (re- } \\ \text { porting bias) }\end{array} & \text { Unclear risk } & \begin{array}{l}\text { Comment: all primary and secondary endpoints assessed and reported. Pub- } \\ \text { lished protocol. Some lack of clarity in protocol regarding choice of measure- } \\ \text { ment tool (original trial record states "patient reported symptoms of sinusitis" } \\ \text { will be assessed, but does not state which tools will be used). }\end{array}\end{array}$

\section{Bachert 2017}

Methods Double-blind, parallel-group RCT with 24 weeks of treatment/follow-up

Participants

Setting: multicentre study at 6 sites in Europe (Belgium, the Netherlands and the UK)

\section{Sample size:}

- Number randomised: 107

- Number completed: 74 (42 in intervention group, 32 in comparator)

Participant (baseline) characteristics:

- Age: mean 51 years mepolizumab group; mean 50 years placebo group

- Gender: $76 \%$ male mepolizumab group; $67 \%$ male placebo group

- Main diagnosis: severe recurrent bilateral nasal polyposis requiring surgery

- Polyps status: bilateral nasal polyp score mean 6.28 mepolizumab group; 6.31 placebo group (range 0 to 8 , higher $=$ worse)

- Previous sinus surgery status: all participants had at least one previous surgery (inclusion criterion)

- Previous courses of steroids: refractory to standard-of-care steroid therapy (received INCS for $\geq 3$ months and/or received a short course of oral steroids) at the time of enrollment

- Asthma: 81\% mepolizumab group; $75 \%$ placebo group

- Need for surgery: all participants were deemed to require surgery at baseline, according to the inclusion criteria (see above)

\section{Inclusion criteria:}

- Diagnosis of severe bilateral nasal polyposis at the screening visit and Visit 1 (i.e. at end of run-in period), which meets the definition of the situation indicative of the need for surgery (an endoscopic nasal polyposis score of 3 or greater and a symptom score of greater than 7 on a VAS)

- At least one previous surgery for the removal of nasal polyps 
- History of refractory response to steroid therapy as shown by being deemed potentially eligible for surgery despite having been on a regular/continuous course of nasal corticosteroids for the treatment of nasal polyposis for at least 3 months and/or have received a short course of oral steroids in the past for nasal polyp treatment

- Male or female between 18 and 70 years of age, inclusive

- BMI within the range 19.0 to $31.0 \mathrm{~kg} / \mathrm{m}^{2}$ (inclusive)

- Free of any clinically significant disease that would interfere with the study schedule or procedures or compromise his/her safety

- Concurrent asthma must be maintained on no more than $10 \mathrm{mg} /$ day of prednisolone or the equivalent

- Adequate contraception

\section{Exclusion criteria:}

- Requiring oral corticosteroids at a dose greater than $10 \mathrm{mg}$ prednisolone or equivalent during the study

- Asthma exacerbation requiring admission to hospital within 4 weeks of screening

- Immunotherapy within the previous 12 months

- Positive pre-study drug/alcohol screen. A minimum list of drugs that will be screened for include amphetamines, barbiturates, cocaine, opiates, cannabinoids and benzodiazepines.

- Known medical history of hepatitis B, hepatitis C or HIV infection

- History or suspicion of drug abuse or alcohol abuse within the last 6 months

- Currently receiving, or have received within 3 months prior to first mepolizumab dose, chemotherapy, radiotherapy or investigational medications/therapies

- One or more of the following abnormal laboratory values:

* serum creatinine $\geq 3$ times institutional upper limit of normal;

* AST or/ALT $\geq 5$ times institutional upper limit of normal;

* Platelet count $<50,000 / \mu \mathrm{L}$

- History of sensitivity to any of the study medications, or components thereof or a history of drug or other allergy that contraindicates their participation. Aspirin-sensitive participants were acceptable.

- History of allergic reaction to anti-IL-5 or other antibody therapy

- Positive serum pregnancy test at screening or positive urine pregnancy test prior to each dosing occasion

- Breastfeeding/lactating

- Current smoker or smoked in the last 6 months

Interventions

Intervention ( $\mathbf{n}=\mathbf{5 4})$ :

- 750 mg intravenous infusion of mepolizumab every 4 weeks for 24 weeks ( 6 doses in total)

Control $(\mathbf{n}=\mathbf{5 3})$ :

- Placebo given intravenously every 4 weeks for 24 weeks ( 6 doses in total)

Use of additional medication (common to both groups): $100 \mu \mathrm{g}$ fluticasone propionate nasal spray in each nostril daily given during a 10- to 14-day run-in period and continued this dose throughout the trial. Inhaled asthma controller therapies could be continued.

All reported at 25 weeks

- Disease-specific health-related quality of life (SNOT-22 score)

- Disease severity symptom score (VAS score range 0 to 10 , "how troublesome are your symptoms of nasal polyposis?", individual VAS scores for four symptoms (rhinorrhoea, mucus in the throat, nasal blockage and loss of smell))

- Severe adverse events

Secondary outcomes (relevant to this review): 
Bachert 2017 (Continued)

All reported at 25 weeks

- Avoidance of surgery (number of participants who no longer met the criteria for requiring surgery)

- Endoscopic nasal polyp score (range 0 to 8, higher = worse)

- Health-related quality of life, generic (EQ-5D scores, scale 0 to 100 , higher = better)

- Nasopharyngitis

Other outcomes reported by the study:

All reported at 25 weeks

- Sense of smell - Sniffin' Sticks Screening-12

- Lung function assessments

\begin{tabular}{|c|c|}
\hline Funding sources & GlaxoSmithKline \\
\hline Declarations of interest & $\begin{array}{l}\text { GlaxoSmithKline, in collaboration with the academic clinical investigators, provided input on the de- } \\
\text { sign and conduct of the study; oversaw the collection, management and statistical analysis of data; } \\
\text { and contributed to the interpretation of the data and the preparation, review and submission of the } \\
\text { manuscript. All authors had roles in the conception, design and interpretation of the analysis. All au- } \\
\text { thors participated in the development of the manuscript and had access to the data from the study. } \\
\text { The decision to submit for publication was that of the authors alone. The final decision on manuscript } \\
\text { submission was made by the authors. The sponsors did not have the right to veto publication. }\end{array}$ \\
\hline
\end{tabular}

Notes Trial registration number NCT01362244

\section{Risk of bias}

Bias Authors' judgement Support for judgement

Random sequence genera- Low risk tion (selection bias)
Quote: "A randomization schedule was generated before the start of the study by using validated internal software. Patients were randomized with the GlaxoSmithKline IVRS system RAMOS. Site staff called the RAMOS system to register the patient on the system and allocated a randomization number. The randomization schedule used by the RAMOS system was generated by the GlaxoSmithKline study statistician before the start of the study using validated internal software. A center-based randomization schedule was used, with blocking (block size 4)."

Comment: central randomisation using computer software

Allocation concealment Low risk
(selection bias)

Quote: "site staff (except for the unblinded pharmacist), GlaxoSmithKline study staff (except for the independent statistician who analyzed the interim data), and bioanalytical staff (placebo-treated subjects were not assayed for PK concentrations) had no access to the random codes until after completion of the study."

Comment: central allocation, separate to enrolment of participants

\begin{tabular}{|c|c|c|}
\hline $\begin{array}{l}\text { Blinding of participants } \\
\text { and personnel (perfor- } \\
\text { mance bias) } \\
\text { All outcomes }\end{array}$ & Low risk & $\begin{array}{l}\text { Quote: "The patients and treating doctors were blind to treatment." } \\
\text { Comment: double-blind }\end{array}$ \\
\hline $\begin{array}{l}\text { Blinding of outcome as- } \\
\text { sessment (detection bias) } \\
\text { All outcomes }\end{array}$ & Low risk & $\begin{array}{l}\text { Quote: "Blinding was strictly maintained until all data had been collected and } \\
\text { cleaned and Database Freeze had been declared." } \\
\text { Comment: blinded study, outcomes collected prior to unmasking }\end{array}$ \\
\hline
\end{tabular}


Bachert 2017 (Continued)

Incomplete outcome data High risk (attrition bias)

All outcomes
Quote: "[for placebo] 32 (63\%) completed treatment phase to Week 25. [for mepolizumab] 42 (78\%) completed treatment phase to Week 25."

Comment: high dropout (>20\%) in both arms, > 10\% difference between the groups. There were high rates of discontinuation, with imbalance between arms (19 (37\%) of placebo group and $12(22 \%)$ of mepolizumab population discontinued), which may impact on results.

Selective reporting (re- Low risk porting bias)

Comment: all primary and secondary endpoints assessed and reported

\section{Gevaert 2011}

Methods Double-blind, parallel-group RCT with 8 weeks of treatment and 40 weeks of follow-up

Participants

Setting: single centre within Europe (Belgium)

Sample size: $\mathbf{3 0}$

- Number randomised: 30

- Number completed: 10 (9 in intervention group, 1 in comparator)

Participant (baseline) characteristics:

- Age: mean 50.0 years mepolizumab group; mean 45.9 years placebo group

- Gender: $70 \%$ male mepolizumab group, $80 \%$ male placebo group

- Main diagnosis: chronic sinusitis with primary nasal polyps (grades 3 or 4) or recurrent nasal polyps (grade 1 to 4 )

- Polyps status: bilateral nasal polyp score mean 5.2 mepolizumab group; mean 5.5 placebo group(range 0 to 8 , higher $=$ worse)

- Previous sinus surgery status: $75 \%$ had $\geq 1$ previous surgery for nasal polyps in mepolizumab group; $80 \%$ in placebo group

- Previous courses of steroids: (excluded if received oral corticosteroids within past month)

- $50 \%$ mepolizumab group and $30 \%$ of placebo group reported comorbid asthma

- $25 \%$ of mepolizumab group and $0 \%$ of placebo group reported aspirin sensitivity

- Need for surgery: no information provided regarding whether participants were deemed to require surgery at baseline (no surgical outcomes reported)

\section{Inclusion criteria:}

- Chronic rhinosinusitis with primary nasal polyps grade 3 to 4 (each nostril scored 0 to 4 , higher $=$ worse) or recurrent nasal polyps after surgery (grade 1 to 4); and

- Failure of standard care for chronic rhinosinusitis with nasal polyps.

\section{Exclusion criteria:}

- Use of systemic corticosteroids/surgery in the month before recruitment

- Use of nasal corticosteroids, nasal antihistamines, nasal atropine, nasal cromolyn, nasal saline or antibiotic treatment for 2 months after first dosing

Interventions

Intervention $(\mathbf{n}=\mathbf{2 0})$ :

- 2 doses of $750 \mathrm{mg}$ dose of intravenous mepolizumab given 28 days apart

Control $(n=10)$ :

- Placebo given IV 28 days apart in 2 doses 
Use of additional medication (common to both groups): use of systemic corticosteroids and surgical intervention was not allowed from 1 month before treatment until the end of the study, and participants were not permitted to use nasal corticosteroids, nasal antihistamines, nasal atropine, nasal cromolyn, nasal saline or antibiotic treatment for 2 months after first dosing.

\section{Outcomes Primary outcomes (relevant to this review):}

- Disease severity symptom scores (4 individual symptoms, anterior rhinorrhoea, nasal obstruction, postnasal drip and loss of sense of smell, each scored with range 0 to 3 , higher $=$ worse) (reported at 8 weeks)

- Serious adverse events (reported at 48 weeks)

\section{Secondary outcomes (relevant to this review):}

- Endoscopy (reduction in nasal polyp score) (reported at 8 weeks)

- Change in CT scan score (improvement versus worsening or no change) (reported at 8 weeks)

- Pharyngitis (reported at 48 weeks)

\section{Other outcomes reported by the study:}

All reported at 8 weeks

- Nasal peak inspiratory flow

- Blood and serum markers (eosinophils, serum IL-5Ra, eosinophil cationic protein)

\begin{tabular}{ll}
\hline Funding sources & Study was supported by GlaxoSmithKline (GSK), who also provided the study drug \\
\hline Declarations of interest & 2 trial authors were employed by GSK and a further 2 authors received funding from GSK \\
\hline Notes & Trial registration number: not available \\
\hline
\end{tabular}

\section{Risk of bias}

Bias Authors' judgement Support for judgement

Random sequence genera- Unclear risk tion (selection bias)
Quote: "Subjects were randomized to receive..."

Comment: no further details given, therefore unclear how randomisation was performed or by whom.

Although not statistically significant, more participants in the intervention arm had asthma and/or aspirin intolerance

Allocation concealment Unclear risk Comment: no information provided
(selection bias)

\begin{tabular}{|c|c|c|}
\hline $\begin{array}{l}\text { Blinding of participants } \\
\text { and personnel (perfor- } \\
\text { mance bias) }\end{array}$ & Low risk & $\begin{array}{l}\text { Quote: "The study was double blind up to } 48 \text { weeks" } \\
\text { Comment: described as double-blind and placebo injection was used }\end{array}$ \\
\hline
\end{tabular}

Unclear risk

Blinding of outcome assessment (detection bias)

All outcomes
Comment: no comment on blinding of outcome assessors. Some subjective outcomes (e.g. worsening/improvement in CT scans).
Incomplete outcome data High risk (attrition bias)

All outcomes
Quote: "At the end of the study there was a considerable drop out rate in both the mepolizumab and placebo arms."

Comment: high dropout (30\%) in placebo arm versus $10 \%$ in intervention arm by week 8 
Gevaert 2011 (Continued)

Selective reporting (reporting bias)
Unclear risk
Comment: no published protocol available. Insufficient detail in methods to judge adequacy of reporting. Some outcome measures reported narratively (e.g. symptom scores), with no data to support the description. No online record identified for CRT110178, so could not compare.

Gevaert 2013

Methods Double-blind, parallel-group, 2-arm RCT with 16 weeks duration of treatment and 4 weeks follow-up

Participants

Setting: 2 centres in European hospitals (Belgium)

Sample size: 24

- Number randomised: 24

- Number completed: 23 (15 in intervention group, 8 in comparator)

Participant (baseline) characteristics:

- Age, median (IQR): 50 (44 to 56) omalizumab group; 45 (42 to 54) placebo group

- Gender, men/women (n): 12/3 omalizumab group; 4/4 placebo group

- Main diagnosis: chronic rhinosinusitis with nasal polyps

- Polyps status (total nasal endoscopic polyp score) median (IQR): 6 (4 to 6) omalizumab group; 6 (6 to 8) placebo group

- Previous sinus surgery status; $\mathrm{n}(\%)$ with previous surgery: 13 (87) omalizumab group; 6 (75) placebo group

- Previous courses of steroids: not reported

- Aspirin hypersensitivity: $12 / 24$ patients

- Asthma: all participants had asthma

- Need for surgery: no information provided regarding whether participants were deemed to require surgery at baseline (no surgical outcomes reported)

\section{Inclusion criteria:}

- Chronic rhinosinusitis (according to the European Position Paper on Rhinosinusitis and Nasal Polyps guidelines) and comorbid asthma (based on Global Initiative for Asthma guidelines and diagnosed by a respiratory physician) for more than 2 years

- Total serum IgE levels between 30 and $700 \mathrm{kU} / \mathrm{mL}$

\section{Exclusion criteria:}

- None stated and none available in online repository

- Subcutaneous treatment with anti-IgE (omalizumab). The dose and dosing frequency (every 2 weeks/8 injections in total or every month/4 injections in total) of omalizumab were based on total serum IgE levels and body weight, with a maximum dose of $375 \mathrm{mg}$. After screening, 10 visits were scheduled every 2 weeks over 20 weeks.

\section{Control $(n=8)$ :}

- Placebo injection, schedule as above

Use of additional medication (common to both groups): maintenance treatment for asthma was standardised and controlled by a respiratory physician. During the study, participants were not permitted to use systemic corticosteroids, an inhaled corticosteroid (doses of greater than $1000 \mu \mathrm{g} / \mathrm{day}$ beclomethasone dipropionate or equivalent), antibiotic treatment, leukotriene receptor antagonists or nasal decongestants. 
Gevaert 2013 (Continued)

Outcomes

\section{Primary outcomes (relevant to this review):}

- Disease-specific health-related quality of life (RSOM-31, AQLQ) (at 16 weeks)

- Disease severity symptom score, nasal and asthma symptoms (patient-reported, daily "absent, mild, moderate or severe" (scores $0,1,2,3$ ) (at 16 weeks)

- Significant adverse effects (unclear time frame, presumed to be at 20 weeks)

\section{Secondary outcomes (relevant to this review):}

All reported at 16 weeks

- Health-related quality of life, generic (SF-36)

- Endoscopy (polyps size or overall score) (total nasal endoscopic polyp score (primary outcome) at 16 weeks)

- CT scan (change in Lund Mackay CT scores)

\section{Other outcomes reported by the study:}

All reported at 16 weeks

- $\mathrm{FEV}_{1}$ and PEFV (percentage of predicted)

- Peripheral blood eosinophil counts, serum total IgE levels and measurement of cytokines and mediators in sera and nasal secretions

Funding sources

This study received an unrestricted grant from Novartis, and Novartis provided the study medication

Research grants from Ghent University and the Flemish Scientific Research Board; the Interuniversity Attraction Poles program (IUAP)-Belgian state-Belgian Science Policy P6/35, and the Global Allergy and Asthma European Network

Declarations of interest Gevaert, Calus, Van Zele, Blomme, De Ruyck and Bachert were provided with medication by Novartis. The rest of the authors declare that they have no relevant conflicts of interest.

Notes Trial registration number: NCT01393340

\section{Risk of bias}

\begin{tabular}{lll}
\hline Bias & Authors' judgement & Support for judgement \\
\hline $\begin{array}{l}\text { Random sequence genera- } \\
\text { tion (selection bias) }\end{array}$ & Low risk & Quote: "computer-generated randomization list " \\
\hline $\begin{array}{l}\text { Allocation concealment } \\
\text { (selection bias) }\end{array}$ & Unclear risk & Quote: "computer-generated randomization list" \\
& $\begin{array}{l}\text { Comment: states "list" with no further information. No details on separation of } \\
\text { individuals who recruit to the study and allocate intervention/placebo. }\end{array}$ \\
$\begin{array}{l}\text { Blinding of participants } \\
\begin{array}{l}\text { and personnel (perfor- } \\
\text { mance bias) }\end{array}\end{array}$ & Unclear risk & Quote: "Both the investigator and the subject were blind to study treatment." \\
All outcomes & & $\begin{array}{l}\text { Comment: low risk if the investigator is also the care provider, but this is not } \\
\text { clear from the publication. }\end{array}$
\end{tabular}

Blinding of outcome as- Unclear risk sessment (detection bias)

All outcomes

\section{Quote: "Polyps were evaluated on each side by means of nasal endoscopy at each visit and graded based on polyp size."}

Comment: unclear whether assessors were blinded to treatment group. Not stated whether investigator (blinded) was also responsible for outcome measurement. Blinding of assessor is clearly stated for other outcomes (CT scan), but not mentioned for this, the primary outcome for the study. 
Gevaert 2013 (Continued)

Incomplete outcome data Low risk (attrition bias)

All outcomes
Quote "All patients completed all study visits."

Comment: 1 dropout prior to medication being given (omalizumab group). All other participants completed follow-up (although some discontinued medication - ITT analysis).

\begin{tabular}{|c|c|c|}
\hline $\begin{array}{l}\text { Selective reporting (re- } \\
\text { porting bias) }\end{array}$ & High risk & $\begin{array}{l}\text { Comment: trial registration NCT01393340 had week } 20 \text { as the endpoint but } \\
\text { publication had } 16 \text { weeks as the endpoint. }\end{array}$ \\
\hline
\end{tabular}

\section{LIBERTY SINUS 24}

Methods

Double-blind, parallel-group RCT with 24 weeks of treatment and 24 weeks of follow-up

Participants

Setting: multicentre study based in 67 hospitals or clinical centres in 13 countries (Bulgaria, Czechia, France, Germany, Hungary, Italy, the Netherlands, Poland, Romania, Ukraine, Russia, the UK and the USA)

Sample size: 276

- Number randomised: 276

- Number completed: 262 (138 in intervention group, 124 in comparator)

Participant (baseline) characteristics:

- Age: mean 52 years dupilumab group; mean 50 years placebo group

- Gender: 62\% male dupilumab group, $63 \%$ male placebo group

- Main diagnosis: bilateral nasal polyps and symptoms of chronic rhinosinusitis despite intranasal corticosteroid therapy before randomisation

- Polyps status: $100 \%$ with polyps. Bilateral endoscopic polyp score 5.64 for dupilumab group, 5.86 for placebo group (scale 0 to 8 , higher = worse)

- Previous sinus surgery status: $69 \%$ of dupilumab group had previous sinus surgery, $74 \%$ of placebo group had previous sinus surgery. Time since most recent surgery, mean 5.93 years for dupilumab group, 5.54 years for placebo group.

- Previous courses of steroids: $64 \%$ of dupilumab group had a course of systemic corticosteroids in the preceding 2 years, $65 \%$ of the placebo group

- Asthma was diagnosed in $57 \%$ of dupilumab group, $59 \%$ of placebo group

- NSAID-exacerbated respiratory disease was diagnosed in $32 \%$ of dupilumab group, $29 \%$ of placebo group

- Other type 2 medical history (non-asthma/NSAID-exacerbated disease) was reported in $57 \%$ of dupilumab group and $56 \%$ of placebo group

- Need for surgery: no information provided regarding whether participants were deemed to require surgery at baseline

\section{Inclusion criteria:}

- $\geq 18$ years of age

- Chronic rhinosinusitis with bilateral nasal polyps

- Prior treatment with systemic glucocorticoids within the last 2 years (or a medical contraindication or intolerance to systemic glucocorticoids), prior surgery for nasal polyps, or both

- Endoscopic bilateral nasal polyp score of at least 5 (out of 8), with a minimum score of 2 in each nasal cavity

- Ongoing symptoms for at least 8 weeks prior to study entry, including:

* nasal congestion, blockage or obstruction with moderate or severe symptom severity (score 2 or 3 ) and a weekly average severity score of at least 1 (range 0 to 3 ) at randomisation; and

* at least one other symptom, such as partial loss of smell (hyposmia), total loss of smell (anosmia), or anterior or posterior rhinorrhoea 
- Patients with concomitant asthma had to be stable in the previous 6 weeks using their regular asthma treatment

\section{Exclusion criteria:}

- Previous participation in a dupilumab study

- Received biologic therapy/systemic immunosuppressant to treat inflammatory or autoimmune disease within 2 months of study entry or 5 half-lives, whichever is longer

- Received experimental monoclonal antibody treatment within 5 half-lives or 6 months of study entry

- Received anti-IgE therapy within 130 days prior to study entry

- Received leukotriene antagonist/modifier treatment unless continuous treatment was received $\geq 30$ days prior to study entry

- Any sinus intranasal surgery (including nasal polypectomy) within 6 months before visit 1

- Patients with a forced expiratory volume in 1 second $\left(\mathrm{FEV}_{1}\right) \leq 50 \%$ of predicted normal (for comorbid asthma patients)

- Presence of antrochoanal nasal polyps; acute rhinosinusitis; upper respiratory infection; allergic granulomatous angiitis/eosinophilic granulomatosis with polyangiitis; granulomatosis with polyangiitis; cystic fibrosis; fungal rhinosinusitis; Young syndrome; Kartagener syndrome; or dyskinetic cilia syndrome

Interventions

\section{Intervention ( $=143)$ :}

- 300 mg subcutaneous dupilumab every 2 weeks for 24 weeks

\section{Control $(n=133)$ :}

- Placebo given subcutaneously every 2 weeks for 24 weeks

Use of additional medication (common to both groups): $100 \mu \mathrm{g}$ mometasone furoate nasal spray in each nostril twice daily given during the 4-week run-in period and throughout the trial. Saline nasal lavage, systemic antibiotics, short-course systemic corticosteroids or sinonasal surgery were permitted as needed during the treatment and follow-up periods.

Outcomes

\section{Primary outcomes (relevant to this review):}

All reported at 24 weeks

- Disease-specific health-related quality of life (SNOT-22 score)

- Disease severity symptom score (VAS for rhinosinusitis, scored 0 to $10 \mathrm{~cm}$ for the questions "how troublesome are your symptoms of rhinosinusitis?"; patient-reported total symptoms score (composite severity score including symptoms of nasal congestion, loss of smell and anterior/posterior rhinorrhoea, each scored 0 to 30) with range 0 to 9 , higher = worse)

- Serious adverse events

\section{Secondary outcomes (relevant to this review):}

All reported at 24 weeks

- Number of participants requiring surgery

- Endoscopic nasal polyp score (range 0 to 8, higher = worse)

- CT scan score (change from baseline in sinus opacification, assessed by Lund Mackay CT score, range 0 to 24 , higher $=$ worse)

- Generic health-related quality of life (EQ-5D score, range 0 to 100 , higher = better)

- Nasopharyngitis

\section{Other outcomes reported by the study:}

All reported at 24 weeks

- Rescue treatment use of corticosteroids (participants with $\geq 1$ event by week 24 )

- Change from baseline in nasal peak inspiratory flow 
LIBERTY SINUS 24 (Continued)

- FEV ${ }_{1}$ and Asthma Control Questionnaire-6 for patients with asthma

- UPSIT score

\begin{tabular}{ll}
\hline Funding sources & Sanofi and Regeneron Pharmaceuticals \\
\hline Declarations of interest & Trial authors employed/received funding from Sanofi and Regeneron Pharmaceuticals \\
\hline Notes & Trial registration number: NCT02912468 \\
\hline
\end{tabular}

\section{Risk of bias}

Bias Authors' judgement Support for judgement

Random sequence genera- Low risk tion (selection bias)
Quote: "Patients were randomly assigned centrally with a permuted block randomisation schedule by Interactive Voice Response System or Interactive Web Response System. Randomisations and allocations were done with use of ClinPhone from Parexel (Waltham, MA, USA), which generated the patient randomisation list and treatment assignment."

Comment: central randomisation using computer software.
Quote: "Randomisations and allocations were done with use of ClinPhone from Parexel (Waltham, MA, USA), which generated the patient randomisation list and treatment assignment. [...]The sponsor provided the randomisation scheme to the centralised treatment allocation system and treatments were allocated to the patients accordingly."

Comment: central allocation, separate to enrolment of participants.
Quote: "both patients and investigators were masked to the assigned drug, with active drug or matching placebo used in identical prefilled syringes labelled with a treatment kit number."

Comment: double-blind

\begin{tabular}{|c|c|c|}
\hline $\begin{array}{l}\text { Blinding of outcome as- } \\
\text { sessment (detection bias) } \\
\text { All outcomes }\end{array}$ & Low risk & $\begin{array}{l}\text { Quote: "Treatment group information was masked in data transfers from } \\
\text { Parexel to the sponsor until database lock. [...] Once all data were clean and } \\
\text { approved by the site, the database was extracted and locked, and data were } \\
\text { transferred to the SAS environment for statistical analysis." }\end{array}$ \\
\hline
\end{tabular}

Comment: blinded study, outcomes reported prior to randomisation code being broken.

Incomplete outcome data Unclear risk
(attrition bias)
All outcomes

Quote: "We did efficacy analyses in the intention-to-treat population, defined as all patients who were randomly assigned; data were analysed according to assigned intervention, whether received or not.[...] 12 (4\%) of 276 patients discontinued treatment before week 24 , and $13(5 \%)$ patients discontinued from the study; one patient was randomly assigned, but not treated, and the primary reason for discontinuation was occurrence of adverse events."

Comment: reasons for dropouts are explicit; $<10 \%$ loss, balanced across groups. Trialists used WOCF and multiple imputation methods to include in the analysis participants who discontinued. Although similar numbers of participants discontinued due to adverse effects before week 24, 25/133 (18.8\%) placebo group had systemic corticosteroid or surgery before week 24 , compared with 10/143 (7\%) dupilumab group, resulting in imbalance between the groups in follow-up data.

\begin{tabular}{|c|c|c|}
\hline $\begin{array}{l}\text { Selective reporting (re- } \\
\text { porting bias) }\end{array}$ & Unclear risk & $\begin{array}{l}\text { Comment: majority of outcomes are reported in full. Some outcome data are } \\
\text { missing from the publication, including the specific number of participants }\end{array}$ \\
\hline
\end{tabular}




\section{LIBERTY SINUS 52}

Methods

Participants
Double-blind, 3-arm parallel-group RCT with 52 weeks of treatment and follow-up

Setting: 117 hospitals or clinical centres in 14 countries (Argentina, Australia, Belgium, Canada, Chile, Israel, Mexico, Portugal, Russia, Spain, Sweden, Turkey, Japan and the USA)

Sample size: 448

- Number randomised: 448

- Number completed: 428 (142 in intervention arm A, 146 in intervention arm B, 140 in comparator)

Participant (baseline) characteristics:

- Age: mean 53 years dupilumab (2-weekly, decreasing to 4-weekly group); mean 51 years dupilumab (2-weekly group); mean 53 years placebo group

- Gender: 60\% male dupilumab (2-weekly, decreasing to 4-weekly group); 65\% male dupilumab (2weekly group); $62 \%$ male placebo group

- Main diagnosis: bilateral nasal polyps and symptoms of chronic rhinosinusitis despite intranasal corticosteroid therapy before randomisation

- Polyps status: $100 \%$ with polyps. Mean bilateral endoscopic polyp score 6.29 for dupilumab (2-weekly, decreasing to 4-weekly group), 6.07 for dupilumab (2-weekly group), 5.96 for placebo group (scale 0 to 8).

- Previous sinus surgery status: $59 \%$ of dupilumab (2-weekly, decreasing to 4-weekly group) had previous sinus surgery, $59 \%$ of dupilumab (2-weekly group) had previous sinus surgery, $58 \%$ of placebo group had previous sinus surgery. Time since most recent surgery, mean 8.41 years for dupilumab (2-weekly, decreasing to 4-weekly group); 7.54 years for dupilumab (2-weekly group); 8.77 years for placebo group

- Previous courses of steroids: $80 \%$ of dupilumab (2-weekly, decreasing to 4-weekly) group had a course of systemic corticosteroids in the preceding 2 years; $81 \%$ of dupilumab (2-weekly) group; $80 \%$ of the placebo group

- Asthma: diagnosed in $63 \%$ of dupilumab (2-weekly, decreasing to 4-weekly group); $57 \%$ of dupilumab (2-weekly) group; $59 \%$ of placebo group

- NSAID-exacerbated respiratory disease: diagnosed in $28 \%$ of dupilumab (2-weekly, decreasing to 4 weekly) group; $23 \%$ of dupilumab (2-weekly) group and $29 \%$ of placebo group.

- Other type 2 medical history: (non-asthma/NSAID-exacerbated disease) was reported in $68 \%$ of dupilumab (2-weekly, decreasing to 4-weekly) group, $64 \%$ of dupilumab (2-weekly) group and $64 \%$ of placebo group

- Need for surgery: no information provided regarding whether participants were deemed to require surgery at baseline

\section{Inclusion criteria:}

- $\geq 18$ years of age

- Chronic rhinosinusitis with bilateral nasal polyps

- Prior treatment with systemic glucocorticoids within the last 2 years (or a medical contraindication or intolerance to systemic glucocorticoids), prior surgery for nasal polyps, or both

- Endoscopic bilateral nasal polyp score of at least 5 (out of 8), with a minimum score of 2 in each nasal cavity 
- Ongoing symptoms for at least 8 weeks prior to study entry, including:

* Nasal congestion, blockage or obstruction with moderate or severe symptom severity (score 2 or 3 ) and a weekly average severity score of at least 1 (range 0 to 3 ) at randomisation; and

* At least one other symptom, such as partial loss of smell (hyposmia), total loss of smell (anosmia), or anterior or posterior rhinorrhoea

- Patients with concomitant asthma had to be stable in the previous 6 weeks using their regular asthma treatment

\section{Exclusion criteria:}

- Previous participation in a dupilumab study

- Received biologic therapy/systemic immunosuppressant to treat inflammatory or autoimmune disease within 2 months of study entry or 5 half-lives, whichever is longer

- Received experimental monoclonal antibody treatment within 5 half-lives or 6 months of study entry

- Received anti-IgE therapy within 130 days prior to study entry

- Received leukotriene antagonist/modifier treatment unless continuous treatment was received $\geq 30$ days prior to study entry

- Any sinus intranasal surgery (including nasal polypectomy) within 6 months before visit 1

- Patients with a forced expiratory volume in 1 second $\left(\mathrm{FEV}_{1}\right) \leq 50 \%$ of predicted normal (in comorbid asthma patients)

- Presence of antrochoanal nasal polyps; acute rhinosinusitis; upper respiratory infection; allergic granulomatous angiitis/eosinophilic granulomatosis with polyangiitis; granulomatosis with polyangiitis; cystic fibrosis; fungal rhinosinusitis; Young syndrome; Kartagener syndrome; or dyskinetic cilia syndrome

Interventions Intervention $(\mathrm{n}=295)$

- Arm A: 300 mg subcutaneous dupilumab every 2 weeks for 24 weeks, followed by every 4 weeks until a total of 52 weeks ( $n=145)$; or

- Arm B: 300 mg subcutaneous dupilumab every 2 weeks for 52 weeks $(n=150)$

Control $(n=153)$

- Placebo given subcutaneously every 2 weeks for 52 weeks

Use of additional medication (common to both groups): $100 \mu \mathrm{g}$ mometasone furoate nasal spray in each nostril twice daily given during the 4-week run-in period and throughout the trial. Saline nasal lavage, systemic antibiotics, short-course systemic corticosteroids or sinonasal surgery were permitted as needed during the treatment and follow-up periods.

Primary outcomes (relevant to this review):

- Disease-specific health-related quality of life (SNOT-22 score) (reported at 24 and 52 weeks)

- Disease symptom severity score (VAS scored 0 to $10 \mathrm{~cm}$, for the question "how troublesome are your symptoms of rhinosinusitis?"; patient-reported total symptoms score (including nasal congestion, loss of smell and anterior/posterior rhinorrhoea, each scored as 0 to 3), range 0 to 9 , higher = worse) (reported at 24 weeks)

- Serious adverse events (reported at 52 weeks)

\section{Secondary outcomes (relevant to this review):}

- Number of participants requiring surgery (reported at 24 weeks)

- Endoscopic nasal polyp score (range 0 to 8, higher = worse) (reported at 24 weeks)

- CT scan score (change from baseline in sinus opacification, assessed by Lund Mackay CT score, range 0 to 24 , higher $=$ worse) (reported at 24 weeks)

- Nasopharyngitis, including sore throat (reported at 52 weeks) 
LIBERTY SINUS 52 (Continued)

Declarations of interest Trial authors employed/received funding from Sanofi and Regeneron Pharmaceuticals

Notes This is a 3-arm trial. Data from the 2 intervention arms were combined for outcomes reported at 24 weeks.

Trial registration number: NCT02898454.

\section{Risk of bias}

Bias Authors' judgement Support for judgement

Random sequence genera- Low risk tion (selection bias)
Quote: "Patients were randomly assigned centrally with a permuted block randomisation schedule by Interactive Voice Response System or Interactive Web Response System. Randomisations and allocations were done with use of ClinPhone from Parexel (Waltham, MA, USA), which generated the patient randomisation list and treatment assignment."

Comment: central randomisation using computer software.

Allocation concealment Low risk
(selection bias)

sch

Quote: "Randomisations and allocations were done with use of ClinPhone
from Parexel (Waltham, MA, USA), which generated the patient randomisation list and treatment assignment.[...] The sponsor provided the randomisation scheme to the centralised treatment allocation system and treatments were allocated to the patients accordingly."

Comment: central allocation, separate to enrolment of participants

$\begin{array}{ll}\begin{array}{l}\text { Blinding of participants } \\ \text { and personnel (perfor- }\end{array} & \text { Low risk } \\ \begin{array}{l}\text { mance bias) } \\ \text { All }\end{array} & \begin{array}{l}\text { Quote: "both patients and investigators were masked to the assigned drug, } \\ \text { with active drug or matching placebo used in identical prefilled syringes la- } \\ \text { belled with a treatment kit number." }\end{array}\end{array}$

All outcomes

For intervention group which switched to four weekly injections: "After Week 24, dupilumab administration was alternated with matched placebo injection every other week up to Week 50."

Comment: study stated as double-blind

Blinding of outcome as- $\quad$ Low risk
sessment (detection bias)

All outcomes

Quote: "Treatment group information was masked in data transfers from Parexel to the sponsor until database lock. [...] Once all data were clean and approved by the site, the database was extracted and locked, and data were transferred to the SAS environment for statistical analysis."

Comment: blinded study, outcomes reported prior to randomisation code being broken.

Incomplete outcome data High risk
(attrition bias)

All outcomes 
LIBERTY SINUS 52 (Continued)

Selective reporting (re- Unclear risk porting bias)
Comment: no outcomes reported for 24- to 52-week follow-up for participants who decreased dupilumab dose to 4-weekly. Some data only reported as pooled analysis with another trial (e.g. number of participants requiring surgery).

- Number randomised: 27

- Number completed: 24 (12 in intervention group, 12 in comparator)

\section{Participant (baseline) characteristics:}

- Age: range 18 to 65

- Gender: $7 / 24$ (29\%) female, $17 / 24$ (71\%) male

- Main diagnosis: chronic rhinosinusitis with nasal polyps

- Polyps status: no information

- Previous sinus surgery status: no information

- Previous courses of steroids: no information

- Other important effect modifiers, if applicable (e.g. aspirin sensitivity, comorbidities of asthma): no information

- Need for surgery: no information provided regarding whether participants were deemed to require surgery at baseline (no surgical outcomes reported)

\section{Inclusion criteria:}

- Age $\geq 18$ years

- Criteria for chronic rhinosinusitis: participants must have (1) at least 2 major criteria (facial pain/pressure or headache, nasal congestion, anterior or posterior nasal drainage, hyposmia/anosmia) for at least 3 consecutive months; (2) an abnormal sinus CT scan in at least 2 sinus areas documented within 3 months of entry or endoscopic evidence of disease

- Participants must have bilateral polypoid disease demonstrated either by CT or endoscopy with evidence of nasal polyps or polypoid mucosa on examination in at least 2 of the following areas: right maxillary sinus, left maxillary sinus, right anterior ethmoid sinus, left anterior ethmoid sinus plus a minimal polyp/polypoid score of 4 on the baseline rhinoscopic examination. (Nasal polyps are defined as discreet polyps visible in the middle meatus area.)

- Positive skin test or in vitro reactivity to a perennial aeroallergen

- Meeting study drug-dosing table eligibility criteria (serum IgE level $\geq 30$ to $\leq 1500 \mathrm{IU} / \mathrm{mL}$ and body weight $\geq 30$ to $\leq 150 \mathrm{~kg}$ )

- Minimum total symptom score of 5 (range of scores 0 to 15) at baseline

\section{Exclusion criteria:}

- Women who are pregnant/nursing/not using approved contraception

- Not meeting clinical criteria for omalizumab

- Taking a beta blocker

- Known sensitivity to Xolair (omalizumab)

- Evidence of acute bacterial exacerbation of rhinosinusitis requiring antibiotics

- Having received antibiotics within 3 weeks of the screening visit 
- Uncontrolled moderate to severe asthma with a recent exacerbation requiring use of systemic steroids burst within 6 weeks of study enrolment (participants receiving a maintenance dose of prednisone of $5 \mathrm{mg} /$ day or less will be allowed provided the dose of prednisone is not changed during the study)

- Uncontrolled recurrent epistaxis within the past 6 weeks

- History of hypogammaglobulinaemia, cystic fibrosis, bronchiectasis, immotile cilia syndrome, systemic granulomatous disease, malignancy (or strong family history of malignancy)

- History of recent cocaine use; cigarette smoking in the past 3 years

- Other serious medical problems or major surgery within 3 months of the screening visit

- Any significant history of non-compliance

- Alcohol or drug abuse/dependence within the past 3 months

- Persistent abnormalities of hepatic, renal or haematologic function, defined as: total bilirubin, SGOT and SGPT > $1.5 \times$ upper limit of normal, creatinine $>2.0 \times$ upper limit of normal, absolute neutrophil count $<1.5 \times 109 / \mathrm{L}$, platelets $<100 \times 109 / \mathrm{L}$

- Using oral or systemic steroid burst within 6 weeks of study enrolment, or any other investigational agent in the 30 days prior to enrolment

\section{Interventions Intervention $(\mathrm{n}=13)$}

- Xolair (omalizumab), administered subcutaneously, every 2 to 4 weeks depending on the patient's baseline serum total IgE level (IU/mL) and body weight $(\mathrm{kg})$. Doses $>150 \mathrm{mg}$ are divided among more than one injection site to limit injections to not more than $150 \mathrm{mg}$ per site. Treatment is for 5 months.

Control $(n=14)$

- Xolair placebo $150 \mathrm{mg}$ to $375 \mathrm{mg}$, administered as above

Use of additional medication (common to both groups): no information provided

Outcomes

\section{Primary outcomes (relevant to this review):}

Reported at 18 weeks (4 months)

- Serious adverse events

\section{Secondary outcomes (relevant to this review):}

Reported at 18 weeks (4 months)

- CT scan (scored using the Zinreich modification of the Lund Mackay scoring system)

- Nasal polyp score

Other outcomes reported by the study:

- None reported

\begin{tabular}{ll}
\hline Funding sources & Massachusetts General Hospital (study sponsor) \\
Genentech, Inc. (collaborator)
\end{tabular}

Declarations of interest Quote: "Principal Investigators are NOT employed by the organization sponsoring the study. There IS an agreement between Principal Investigators and the Sponsor (or its agents) that restricts the PI's rights to discuss or publish trial results after the trial is completed"

Notes Trial registration number: NCT01066104

\section{Risk of bias}




\section{NCT01066104 (Continued)}

Random sequence genera- Unclear risk Comment: no information given on method of randomisation, just stated to tion (selection bias)

have "randomized" allocation

\begin{tabular}{lll}
\hline $\begin{array}{l}\text { Allocation concealment } \\
\text { (selection bias) }\end{array}$ & Unclear risk
\end{tabular}

\begin{tabular}{|c|c|c|}
\hline $\begin{array}{l}\text { Blinding of participants } \\
\text { and personnel (perfor- }\end{array}$ & Low risk & $\begin{array}{l}\text { Quote: "Placebo of similar volume and frequency, administered by subcuta- } \\
\text { neous injection." }\end{array}$ \\
\hline All outcomes & & $\begin{array}{l}\text { Comment: triple masking included participants and care providers; placebo } \\
\text { was matching injection }\end{array}$ \\
\hline
\end{tabular}

\begin{tabular}{|c|c|c|}
\hline $\begin{array}{l}\text { Blinding of outcome as- } \\
\text { sessment (detection bias) } \\
\text { All outcomes }\end{array}$ & Low risk & $\begin{array}{l}\text { Comment: triple masking (participant, care provider, investigator); not clear if } \\
\text { "investigator" included outcome assessors, but matching placebo used so un- } \\
\text { likely that they were aware }\end{array}$ \\
\hline
\end{tabular}

\begin{tabular}{lll}
\hline $\begin{array}{l}\text { Incomplete outcome data } \\
\text { (attrition bias) } \\
\text { All outcomes }\end{array}$ & Low risk & $\begin{array}{l}\text { Comment: low attrition, similar between groups: } 1 / 13 \text { in omalizumab group } \\
\text { and } 1 / 14 \text { in placebo group withdrew due to adverse effects, and one person in } \\
\text { placebo group withdrew due to a protocol violation }\end{array}$ \\
\hline $\begin{array}{l}\text { Selective reporting (re- } \\
\text { porting bias) }\end{array}$ & High risk & $\begin{array}{l}\text { Quote: "Total symptom score (TSS) recorded daily. CRS Facial Pain/Headache } \\
\text { questionnaire at each visit." }\end{array}$ \\
& $\begin{array}{l}\text { Comment: methods section states that these outcomes will be collected, but } \\
\text { there are no data presented on clinical trials register entry. No full publication } \\
\text { available. }\end{array}$ \\
\hline
\end{tabular}

\section{Pinto 2010}

Methods Double-blind, parallel-group RCT with 26 weeks treatment/follow-up

Participants

Setting: single-centre study in the USA

\section{Sample size: 14}

- Number randomised: 14

- Number completed: 14 (7 in intervention group, 7 in comparator)

\section{Participant (baseline) characteristics:}

- Age (mean $\pm \mathrm{SD})$ : omalizumab $43.1 \pm 9.8$; placebo $48.6 \pm 9.1$

- Gender (\% male (n/N)): omalizumab 43\% (3/7) 100\% (7/7); placebo 100\% (7/7)

- Main diagnosis: chronic rhinosinusitis

- Polyps status: $7 / 7$ in omalizumab and 5/7 in placebo had nasal polyposis

- Previous sinus surgery status: $100 \%$ had undergone endoscopic sinus surgery

- Previous courses of steroids:

- Intranasal steroids: omalizumab group: $71 \%$ (4/7); placebo group $71 \%(5 / 7)$

- Systemic steroids omalizumab group: $43 \%$ (3/7); placebo group $0 \%(0 / 7)$

- Inhaled asthma therapy taken by $72 \%(5 / 7)$ in omalizumab group and $43 \%(3 / 7)$ in placebo group

- Need for surgery: all participants had undergone endoscopic sinus surgery (no surgical outcomes reported)

\section{Inclusion criteria:}

- Chronic rhinosinusitis was defined by symptoms (nasal obstruction, nasal discharge, facial pain, hyposmia) for greater than 12 weeks, confirmatory findings on nasal endoscopy, and evidence of inflammation on sinus CT scan 
Pinto 2010 (Continued)

- Age 18 to 75 years

- Chronic sinusitis, as defined by symptoms for greater than 12 weeks, despite treatment

- Paranasal sinus CT scan showing evidence of chronic sinusitis

- Positive skin or RAST test to an inhalant allergen

- Serum total IgE between 30 and $700 \mathrm{IU} / \mathrm{mL}$

- Body weight less than $150 \mathrm{~kg}$

- Impaired quality of life, as measured by the Rhinosinusitis Disability Index (RSDI)

\section{Exclusion criteria:}

- Women who are breastfeeding or of childbearing potential not using a contraception method

- Known sensitivity to Xolair

- Patients with severe medical condition(s)

- Use of any other investigational agent in the last 30 days

- No measurable disability on the RSDI

- Immunocompromised patients or patients with ciliary disorders

Interventions

Intervention ( $\mathbf{n}=\mathbf{7})$ :

- Omalizumab administered subcutaneously, once or twice monthly (dose dependent on participant weight and serum IgE level), for 6 months

\section{Control $(n=7)$ :}

- Placebo subcutaneous injection, dosing as for omalizumab

Use of additional medication (common to both groups): rescue medications permitted (trial reported use of courses of systemic steroids, antibiotics and added adjunctive medications (anti-leukotrienes, antihistamines or intranasal steroids)

Outcomes

\section{Primary outcomes (relevant to this review):}

All reported at 26 weeks

- Health-related quality of life, disease specific: SNOT-20, recorded monthly for 6 months; Rhinosinusitis Disability Index (RSDI) recorded monthly for 6 months

- Disease severity symptom score: participants recorded symptoms daily (nasal obstruction, nasal discharge, facial pain and hyposmia) each recorded on a 4-point scale ( $0=$ none, $1=$ mild, $2=$ moderate, 3 = severe); total scores were summed for a TNSS)

\section{Secondary outcomes (relevant to this review):}

All reported at 26 weeks

- Health-related quality of life, generic: SF-36 at 6 months

- Endoscopy (polyps size or overall score): nasal endoscopy score at 6 months

- CT scan - mucosal thickness on CT scan at 6 months (primary outcome)

- Adverse events

\section{Other outcomes reported by the study:}

- Number of sinusitis exacerbations requiring additional treatment at 6 months

- Nasal peak inspiratory flow at 6 months

- Nasal lavage eosinophils at 6 months

- University of Pennsylvania Smell Identification Test (UPSIT) at 6 months

Funding sources

Quote: "Supported in part by a grant from Genentech and the McHugh Otolaryngology Research Fund. JMP was supported by a Dennis W. Jahnigen Career Development Award from the American Geriatrics Society." 
Pinto 2010 (Continued)

NCT record also lists Novartis Pharmaceuticals as a collaborator.

Declarations of interest Quote: "The investigators had full access to all the data in the study and JMP takes responsibility for the integrity of the data and the accuracy of the data analysis."

Notes

Study terminated early. "Patients were monitored after each injection based on prevailing guidelines. These changed during the study to the current recommendation which is 2 hours of observation following the first 3 injections due to new FDA warnings regarding the possible risk of anaphylaxis ... This requirement ended recruitment because of the time commitment required for participation in the study by volunteers."

Comment: early termination resulted in very low number of participants (only 14/50 planned number).

Trial registration number: NCT00117611

\section{Risk of bias}

\begin{tabular}{|c|c|c|}
\hline Bias & Authors' judgement & Support for judgement \\
\hline $\begin{array}{l}\text { Random sequence genera- } \\
\text { tion (selection bias) }\end{array}$ & Unclear risk & $\begin{array}{l}\text { Quote: "... randomized to omalizumab or placebo groups" } \\
\text { Comment: no further details given }\end{array}$ \\
\hline $\begin{array}{l}\text { Allocation concealment } \\
\text { (selection bias) }\end{array}$ & Unclear risk & Comment: no details given \\
\hline $\begin{array}{l}\text { Blinding of participants } \\
\text { and personnel (perfor- } \\
\text { mance bias) } \\
\text { All outcomes }\end{array}$ & Low risk & $\begin{array}{l}\text { Quote: "Subjects were randomized and followed throughout the trial in a } \\
\text { blinded fashion." (main paper); "Masking: Double (Participant, Investiga- } \\
\text { tor)" (NCT record) } \\
\text { Comment: placebo used and trial described as double-blind }\end{array}$ \\
\hline $\begin{array}{l}\text { Blinding of outcome as- } \\
\text { sessment (detection bias) } \\
\text { All outcomes }\end{array}$ & Unclear risk & $\begin{array}{l}\text { Quote "All CT scan (sic) were read blinded to treatment category." } \\
\text { Comment: no comment on blinding for nasal endoscopy outcome. Insufficient } \\
\text { information to judge adequacy of blinding for patient reported outcomes. }\end{array}$ \\
\hline $\begin{array}{l}\text { Incomplete outcome data } \\
\text { (attrition bias) } \\
\text { All outcomes }\end{array}$ & Unclear risk & $\begin{array}{l}\text { Comment: } 0 \text { withdrawals, but } 1 / 7 \text { placebo participant's CT scans could not } \\
\text { be analysed for technical reasons. Given the low number of participants, this } \\
\text { could introduce bias for the primary outcome. }\end{array}$ \\
\hline $\begin{array}{l}\text { Selective reporting (re- } \\
\text { porting bias) }\end{array}$ & Unclear risk & $\begin{array}{l}\text { Comment: outcomes mostly match those in NCT trial registration. RSDI (listed } \\
\text { on NCT) does not appear to have been reported. Report states that no side ef- } \\
\text { fects or adverse events occurred, but no information given on how these were } \\
\text { detected. }\end{array}$ \\
\hline
\end{tabular}

AQLQ: Asthma Quality of Life Questionnaire

AST: aspartate transaminase

ALT: alanine transaminase

BMI: body mass index

CT: computerised tomography

$\mathrm{FEV}_{1}$ : forced expiratory volume in one second

IgE: immunoglobulin $\mathrm{E}$

IQR: interquartile range

ITT: intention-to-treat

IV: intravenous

INCS: intranasal corticosteroids

mAb: monoclonal antibody

NPIF: nasal peak inspiratory flow 
NSAID: non-steroidal anti-inflammatory drug

OCS: oral corticosteroids

PEFV: partial expiratory flow volume

RAST: radioallergosorbent test

$\mathrm{RCT}$ : randomised controlled trial

RSDI: Rhinosinusitis Disability Index

RSOM-31: Rhinosinusitis Outcome Measures-31

SD: standard deviation

SGOT: serum glutamic oxaloacetic transaminase

SGPT: serum glutamic pyruvic transaminase

SNOT-22: Sino-Nasal Outcome Test-22

TNSS: total nasal symptom score

UPSIT: University of Pennsylvania Smell Identification Test

VAS: visual analogue scale

WOCF: worst observation carried forward

Characteristics of excluded studies [ordered by study ID]

\begin{tabular}{|c|c|}
\hline Study & Reason for exclusion \\
\hline Boguniewicz 2019 & STUDY DESIGN: not a RCT \\
\hline Castro 2011 & $\begin{array}{l}\text { POPULATION: less than half had chronic rhinosinusitis and not stratified for chronic rhinosi- } \\
\text { nusitis at randomisation }\end{array}$ \\
\hline De Schryver 2015 & STUDY DESIGN: not a RCT \\
\hline Gevaert 2006 & INTERVENTION: single dose, not a course of treatment \\
\hline Gevaert 2008 & STUDY DESIGN: not a RCT \\
\hline Gonzalez-Diaz 2014 & STUDY DESIGN: not a RCT \\
\hline Hellings 2017 & STUDY DESIGN: not a RCT \\
\hline Laidlaw 2019 & STUDY DESIGN: not a RCT \\
\hline Liberty Asthma Quest & POPULATION: chronic rhinosinusitis diagnosis was self-reported and less than half had it \\
\hline MUSCA & POPULATION: asthma \\
\hline Naclerio 2017 & STUDY DESIGN: not a RCT \\
\hline NCT00603785 & Study withdrawn \\
\hline NCT01285323 & POPULATION: asthma \\
\hline NCT02170337 & POPULATION: safety study in healthy patients \\
\hline NCT02734849 & Study withdrawn \\
\hline NCT02743871 & STUDY DESIGN: not a RCT \\
\hline Perez De Llano 2018 & STUDY DESIGN: not a RCT \\
\hline Tajiri 2013 & STUDY DESIGN: not a RCT \\
\hline
\end{tabular}




\section{Study Reason for exclusion}

Zangrilli 2019

STUDY DESIGN: not a RCT

$\mathrm{RCT}$ : randomised controlled trial

Characteristics of ongoing studies [ordered by study ID]

\section{NCT02772419} $\begin{array}{ll}\text { Trial name or title } & \begin{array}{l}\text { A phase 2, double-blind, placebo-controlled study of benralizumab (KHK4563) in patients with } \\ \text { eosinophilic chronic rhinosinusitis }\end{array}\end{array}$

Methods Double-blind, parallel-group, randomised controlled trial

Participants $\quad$ Adults (20 to 75 years) with:

- Eosinophilic chronic rhinosinusitis with a total score of $\geq 11$ according to the diagnosis of eosinophilic chronic rhinosinusitis at enrollment

- A minimum bilateral nasal polyp score of 3 out of the maximum score of 8 (with a score of at least 1 out of the maximum score of 4 for each nostril) at screening and at enrollment

\section{Interventions}

Outcomes

\section{Benralizumab}

Primary outcome measures:

1. The change from baseline in nasal polyp score at week 12 (time frame: baseline and 12 weeks post-dose)

Secondary outcome measures:

1. The change from baseline in nasal polyp score (time frame: pre-dose and 4, 8, 12, 16, 20, 24 weeks post-dose)

2. The change from baseline in computed tomography (CT) score (time frame: baseline and 12 weeks post-dose)

3. Number of participants discontinued from the study due to aggravation of eosinophilic chronic rhinosinusitis (time frame: up to 24 weeks after dosing)

4. Time to discontinuation (days) from the study due to aggravation of eosinophilic chronic rhinosinusitis (time frame: up to 24 weeks after dosing)

5. The change from baseline in blood eosinophil count (time frame: pre-dose and 4, 8, 12, 16, 20,24 weeks post-dose)

6. The change from baseline in nasal airway resistance (time frame: pre-dose and 4, 8, 12, 24 weeks post-dose). Nasal airway resistance $\left(\mathrm{Pa} / \mathrm{cm}^{3} / \mathrm{s}\right)$.

7. The change from baseline in the averaged values of the olfactory thresholds (time frame: predose and 4, 8, 12, 24 weeks post-dose); olfactory thresholds are assessed by T\&T Olfactometer Test Score ( 5 kinds of smell with eight ( 5 to -2 ) phases)

8. The change from baseline in the improvement of olfactory dysfunction (time frame: pre-dose and $4,8,12,24$ weeks post-dose); olfactory dysfunction ( 1 to 5 ) is calculated by the olfactory thresholds

9. The change from baseline in Sino-Nasal Outcome Test-2 (SNOT-22) (time frame: pre-dose and 4, 8 , 12, 16, 20, 24 weeks post-dose); symptom scores are assessed by VAS (nasal congestion, anterior and posterior nasal drip, loss of the sense of smell, headache and impairment in activities of daily living)

10.The change from baseline in symptom score by visual analogue scale (VAS) (time frame: pre-dose and 4, 8, 12, 16, 20, 24 weeks post-dose); symptom scores are assessed by VAS (nasal congestion, anterior and posterior nasal drip, loss of the sense of smell, headache and impairment in activities of daily living) 
NCT02772419 (Continued)

11.Incidence of treatment-emergent adverse events (TEAEs) or drug-related TEAEs and their nature (time frame: up to 24 weeks after dosing)

\begin{tabular}{ll}
\hline Starting date & - \\
\hline Contact information & - \\
\hline Notes & Actual completion date: March 2017 \\
& Expected publication date: unknown \\
& $\begin{array}{l}\text { Company contacted } 6 \text { January 2020. Response: publication planned. Company response: unable to } \\
\text { provide study data or Clinical Study Report. Email in Appendix 4. }\end{array}$ \\
\hline
\end{tabular}

\section{NCT02799446}

\begin{tabular}{|c|c|}
\hline Trial name or title & NCT02799446 \\
\hline Methods & Randomised controlled trial \\
\hline Participants & $\begin{array}{l}\text { Adults (18 to } 75 \text { years) and a diagnosis of chronic rhinosinusitis according to the clinical practice } \\
\text { guideline (update) of the American Academy of Otolaryngology - Head and Neck Surgery }\end{array}$ \\
\hline Interventions & Reslizumab 3 mg/kg intravenous (IV) \\
\hline \multirow[t]{7}{*}{ Outcomes } & Primary outcome measures: \\
\hline & 1. Change in computed tomography (CT) score (time frame: 24 weeks) \\
\hline & Secondary outcome measures: \\
\hline & 1. Quality of life questionnaire (time frame: 24 weeks) \\
\hline & 2. Smell test (time frame: 24 weeks) \\
\hline & 3. Endoscopy score (time frame: 24 weeks) \\
\hline & 4. Adverse events by body system (time frame: 24 weeks) \\
\hline
\end{tabular}

\begin{tabular}{ll}
\hline Starting date & June 2016 \\
\hline Contact information & - \\
\hline Notes & Expected study completion date: July 2019 \\
& Expected publication: July 2020 \\
& Publication of study results not required until July 2020 \\
\hline
\end{tabular}

NCT03450083

\begin{tabular}{ll}
\hline Trial name or title & NCT03450083 \\
\hline Methods & Randomised controlled trial \\
\hline Participants & Adults (18 to 75 years) with: \\
& S Severe bilateral nasal polyps with average endoscopic score of at least 5
\end{tabular}


NCT03450083 (Continued)

- At least $1000 \mathrm{mg}$ prednisone (or equivalent) over the previous 12 months to control symptoms

- At least 1 prior nasal surgical polypectomy

\begin{tabular}{|c|c|}
\hline Interventions & $30 \mathrm{mg}$ benralizumab will be delivered subcutaneously \\
\hline \multirow[t]{11}{*}{ Outcomes } & Primary outcome measures: \\
\hline & $\begin{array}{l}\text { 1. Nasal polyp size (time frame: } 24 \text { weeks); reduction in endoscopic nasal polyp score after } 6 \text { months } \\
\text { of treatment }\end{array}$ \\
\hline & Secondary outcome measures: \\
\hline & $\begin{array}{l}\text { 1. Nasal polyp size by CT (time frame: } 24 \text { weeks). Lund Mackay (LM) CT scan of sinus will be used to } \\
\text { determine nasal polyp size. Each of } 4 \text { sinuses are graded } 0 \text { to } 3 \text { on each side (total range } 0 \text { to 24; } \\
0 \text { no abnormality) } \\
\text { a. (partial opacification); or }\end{array}$ \\
\hline & b. (complete opacification). \\
\hline & $\begin{array}{l}\text { 2. Clinical survey (time frame: } 24 \text { weeks). Sino-nasal Outcome Test (SNOT-22) nasal symptoms score; } \\
22 \text { questions each scored } 0 \text { to } 5 \text { (no problem - as bad as it can be) for a total range of } 0 \text { to } 110\end{array}$ \\
\hline & $\begin{array}{l}\text { 3. Smell test (time frame: } 24 \text { weeks). UPSIT smell test; } 40 \text { questions with } 4 \text { choices each - number of } \\
\text { correct answers range } 0 \text { to } 40\end{array}$ \\
\hline & $\begin{array}{l}\text { 4. Blood test (time frame: } 24 \text { weeks). Complete blood count (CBC) to determine absolute eosinophil } \\
\text { count; range } 30 \text { to } 300 / \mu \mathrm{L}\end{array}$ \\
\hline & $\begin{array}{l}\text { 5. Rescue medication use (time frame: up to } 24 \text { weeks). Rescue medication score; rescue medica- } \\
\text { tions include triamcinolone twice daily and prednisone } 20 \mathrm{mg} \text { for } 5 \text { days, which will be given only } \\
\text { as needed periodically. Score ranges from } 0 \text { to } 20(0=\text { none, } 5=\text { triamcinolone nasal daily, } 10= \\
\text { triamcinolone nasal twice daily, } 20=\text { prednisone } 20 \mathrm{mg} \text { for } 5 \text { days) }\end{array}$ \\
\hline & $\begin{array}{l}\text { 6. Time to surgery (time frame: } 24 \text { weeks). Time to nasal polyp surgery; measured in months starting } \\
\text { after last injection }\end{array}$ \\
\hline & $\begin{array}{l}\text { 7. Dropout rate (time frame: up to } 24 \text { weeks). Dropout rate; calculated continuously throughout the } \\
\text { study up to } 24 \text { weeks }\end{array}$ \\
\hline
\end{tabular}

Starting date July 2017

Contact information -

Notes $\quad$ Expected completion date: December 2019

Expected publication date: December 2020

Publication of study results not required until December 2020

\section{NCT03614923}

\begin{tabular}{ll}
\hline Trial name or title & NCT03614923 \\
\hline Methods & Randomised controlled trial \\
\hline Participants & Adults (18 to 65 years) with: \\
& Clinically confirmed diagnosis of chronic rhinosinusitis with nasal polyps \\
& Nasal polyp score $\geq 5$ out of a maximum score for both nostrils (with at least a score of 2 for each \\
& nostril) \\
SNOT-22 score $>7$
\end{tabular}


NCT03614923 (Continued)

- Presence of at least 2 of the following symptoms prior to screening: nasal blockade/obstruction/congestion or nasal discharge (anterior/posterior nasal drip); facial pain/pressure; reduction or loss of smell

\begin{tabular}{|c|c|}
\hline Interventions & Etokimab \\
\hline \multirow[t]{7}{*}{ Outcomes } & Primary outcome measures: \\
\hline & $\begin{array}{l}\text { 1. Change from baseline in nasal polyp score (NPS) to week } 16 \text { (time frame: week } 16) \text {. Total scoring } \\
0 \text { to } 8 \text {, scoring of } 0 \text { to } 4(0=\text { no polyps, } 4=\text { large polyps causing complete obstruction) bilateral }\end{array}$ \\
\hline & $\begin{array}{l}\text { 2. Change from baseline in Sino-Nasal Outcome Test }-22 \text { (SNOT-22). Score from week } 16 \text { (time frame: } \\
\text { week } 16) \text {; total scoring } 0 \text { to } 110 \text {, scoring of } 0 \text { to } 5(0=\text { no problem, } 5=\text { problem as bad as it can } \\
\text { be) ( } 22 \text { items) }\end{array}$ \\
\hline & Secondary outcome measures; \\
\hline & 1. Change from baseline in smell test from week 16 (time frame: week 16) \\
\hline & 2. Change from baseline in nasal peak inspiratory flow from week 16 (time frame: week 16) \\
\hline & $\begin{array}{l}\text { 3. Change in sinus opacification as assessed by CT scan using the Lund Mackay score (time frame: } \\
\text { week 16). Total scoring of } 0 \text { to } 24 \text {, ostiomeatal complex } 0 \text { or } 2 \text { (obstructed) for each sinus group } \\
\text { (6), bilateral }\end{array}$ \\
\hline
\end{tabular}

\begin{tabular}{ll}
\hline Starting date & December 2018 \\
\hline Contact information & - \\
\hline
\end{tabular}

Notes $\quad$ Expected completion date: December 2019

Expected publication date: December 2020

Publication of study results not required until December 2020

OSTRO

\begin{tabular}{|c|c|}
\hline Trial name or title & OSTRO (NCT03401229) \\
\hline Methods & Randomised controlled trial \\
\hline \multirow[t]{7}{*}{ Participants } & Adults (18 to 75 years): \\
\hline & $\begin{array}{l}\text { 1. Patients with bilateral sinonasal polyposis that, despite treatment with a stable dose of intranasal } \\
\text { corticosteroids (INCS) for at least } 4 \text { weeks prior to V1, in addition to history of treatment with sys- } \\
\text { temic corticosteroids (SCS - oral, parenteral) or prior surgery for nasal polyposis (NP), have sever- } \\
\text { ity consistent with a need for surgery as described by: a minimum total Nasal Polyp Score (NPS) } \\
\text { of } 5 \text { out of a maximum score of } 8 \text { (with a unilateral score of at least } 2 \text { for each nostril) at V1, and } \\
\text { continuously maintained at V2 to meet the randomisation criterion, as determined by the study } \\
\text { Imaging Core Lab; ongoing symptoms for at least } 12 \text { weeks prior to V1; patient-reported moderate } \\
\text { to severe nasal blockage score (NBS) } 2 \text { or } 3 \text { over the } 2 \text { weeks prior to V1 (2-week recall assessment } \\
\text { of symptoms, scores } 0=\text { none to } 3=\text { severe) }\end{array}$ \\
\hline & $\begin{array}{l}\text { 2. SNOT-22 total score } \geq 30 \text { at enrolment. Patient must meet the following criteria at the randomi- } \\
\text { sation visit: }\end{array}$ \\
\hline & $\begin{array}{l}\text { - At least } 8 \text { days of evaluable daily diary data in the } 14 \text {-day period prior to randomisation (baseline } \\
\text { bi-weekly mean score collected from study Day }-13 \text { to study Day } 0 \text { ) }\end{array}$ \\
\hline & - At randomisation, a bi-weekly mean NBS $\geq 1.5$ \\
\hline & - SNOT-22 total score $\geq 30$ at randomisation \\
\hline & - At least $70 \%$ compliance with INCS during the run-in period based on daily diary \\
\hline
\end{tabular}


OSTRO (Continued)

Interventions
Benralizumab 30 mg subcutaneous
Outcomes
Primary outcome measures:

1. Effect of benralizumab on nasal polyp burden (time frame: week 56 (visit 11)). Change from baseline in endoscopic total nasal polyp score (NPS). NPS (maximum 8) is the sum of the right and left nostril scores

2. Effect of benralizumab on patient-reported nasal blockage (NB) (time frame: week 56 (visit 11)). Change from baseline in mean nasal blockage score (NBS). NBS is assessed in daily diary by asking patients to rate the severity of their worst nasal blockage over the past 24 hours using the following response options: $0=$ none; 1 = mild; 2 = moderate; 3 = severe

Secondary outcome measures:

1. Effect of benralizumab on disease specific health-related quality of life (HRQL) (time frame: week 56 (visit 11)). Change from baseline in SinoNasal Outcome Test (SNOT-22) score. SNOT-22 captures patient-reported physical problems, functional limitations and emotional consequences of sinonasal condition. Its patient-reported symptom severity and symptom impact over the past 2 weeks and are captured via a 6 -point scale $(0=$ no problem to $5=$ problem as bad as it can be). The total score is the sum of item scores and has a range from 0 to 110 .

2. Effect of benralizumab on nasal polyp surgery (time frame: by week 56 (visit 11)). Time to first nasal polyp surgery.

3. Proportion of nasal polyp surgery (time frame: by week 56 (visit 11)). Proportion of patients with surgery for nasal polyps.

4. Systemic corticosteroids (SCS) use for relief of nasal symptoms (time frame: by week 56 (visit 11)). Proportion of patients with SCS use for nasal polyps.

5. Systemic corticosteroids (SCS) use for relief of nasal symptoms (time frame: by week 56 (visit 11)). Time to first SCS course for nasal polyps.

6. Symptoms associated with nasal polyps (time frame: week 56 (visit 11)). Change from baseline in nasal symptom score(s) as captured in the daily diary. Patients report the severity of symptom related to nasal polyps at its worst using a 4-point verbal rating scale ( $0=$ none to $3=$ severe).

7. Symptoms associated with nasal polyps (time frame: week 56 (visit 11)). Sense of smell captured as change from baseline in University of Pennsylvania Smell Identification Test (UPSIT) score. It is a quantitative test of olfactory function which uses microencapsulated odorants that are released by scratching standardised odour-impregnated test booklets. Four booklets each with 10 odorants each are used for the test. Patients are asked to identify the odour using multiple choice format which lists different possibilities. Scores are based on number of correctly identified odours (score range 0 to 40 ).

8. Sinus opacification by computed tomography (CT) scan (subset of patients) (time frame: week 56 (visit 11)). Change from baseline in Lund Mackay score.

9. Patient-reported general health status (time frame: week 56 (visit 11)). Change from baseline in Short Form 36-item Health survey, Version 2 (SF-36v2).

10.Systemic corticosteroids (SCS) use for relief of nasal symptoms (time frame: by week 56 (visit 11)). Total SCS dose used.

11.Systemic corticosteroids (SCS) use for relief of nasal symptoms (time frame: by week 56 (visit 11)). Number of courses of SCS for nasal polyps.

12.Systemic corticosteroids (SCS) use for relief of nasal symptoms (time frame: by week 56 (visit 11)). Total duration of SCS use for nasal polyps.

13.Sinus opacification by computed tomography (CT) scan (subset of patients) (time frame: week 56 (visit 11)). Change from baseline in sinus severity score by Quantitative CT analysis.

\section{Starting date}

January 2018

\section{Contact information}

Expected completion date: August 2020

Expected publication date: August 2021 
POLYP 1

\begin{tabular}{ll}
\hline Trial name or title & POLYP 1 (NCT03280550) \\
\hline Methods & Randomised controlled trial \\
\hline Participants & Adults (18 to 75 years) with:
\end{tabular}

- Nasal polyp score (NPS) $\geq 5$, with a unilateral score of $\geq 2$ for each nostril, at screening (Day -35) and on Day -7

- Sino-Nasal Outcome Test-22 (SNOT-22) score $\geq 20$ at screening (Day -35) and at randomisation (Day 1)

- Treatment with at least nasal mometasone $200 \mu \mathrm{g}$ per day, or equivalent daily dosing of INCS for at least 4 weeks before screening (Day -35)

- Treatment with nasal mometasone $200 \mu \mathrm{g}$ twice a day (or once a day if intolerant to twice daily) during the run-in period with an adherence rate of at least $70 \%$

- Presence of nasal blockage/congestion with NCS $\geq 2$ (1-week recall) at Day -35 and an average of the daily NCS score over the 7 days prior to randomization of NCS $>1$ with at least one of the following symptoms prior to screening: nasal discharge (anterior/posterior nasal drip) and/or reduction or loss of smell

Interventions Omalizumab

Outcomes

Primary outcome measures:

1. Change from baseline in average daily nasal congestion score (NCS) at week 24 (time frame: baseline, week 24)

2. Change from baseline in nasal polyp score (NPS) to week 24 (time frame: baseline, week 24)

Secondary outcome measures:

1. Change from baseline in average daily total nasal symptom score (TNSS) at week 24 (time frame: baseline, week 24)

2. Change from baseline in average daily sense of smell score at week 24 (time frame: baseline, week 24)

3. Change from baseline in average daily posterior rhinorrhoea score at week 24 (time frame: baseline, week 24)

4. Change from baseline in average daily anterior rhinorrhoea score at week 24 (time frame: baseline, week 24)

5. Change from baseline in participant reported health-related quality of life (HRQL) as assessed by the total Sino-Nasal Outcome Test (SNOT)-22 at week 24 (time frame: baseline, week 24)

6. Change from baseline in sense of smell, as assessed by the University of Pennsylvania Smell Identification Test (UPSIT) at week 24 (time frame: baseline, week 24)

7. Change from baseline in Asthma Quality of Life Questionnaire (AQLQ) of $\geq 0.5$ (in participants with comorbid asthma only) at week 24 (time frame: baseline, week 24)

8. Change from baseline in average daily NCS at week 16 (time frame: baseline, week 16)

9. Change from baseline in NPS at week 16 (time frame: baseline, week 16)

10.Percentage of participants with reduction in the need for surgery by week 24 , as defined by a NPS of $\leq 4$ (unilateral score of $\leq 2$ on each side) and improvement in SNOT-22 score of $\geq 8.9$ (time frame: up to week 24)

11.Percentage of participants requiring of rescue treatment (systemic corticosteroid for $\geq 3$ consecutive days) or having had surgery for nasal polyps through week 24 (time frame: up to week 24)

12.Percentage of participants requiring of rescue treatment (systemic corticosteroid for $\geq 3$ consecutive days) through week 24 (time frame: up to week 24) 
POLYP 1 (Continued)

13.Percentage of participants having had surgery for nasal polyps through week 24 (time frame: up to week 24)

14.Percentage of participants with adverse events (time frame: up to week 28)

15.Percentage of participants with serious adverse events (time frame: up to week 28)

16.Percentage of participants with adverse events leading to omalizumab/placebo discontinuation (time frame: up to week 28)

17.Percentage of participants with clinically significant change in laboratory values (time frame: up to week 28)

18.Serum concentration of omalizumab at specified time points (time frame: Day 1, Day 112, Day 168, Day 196)

19.Serum concentration of total and free immunoglobulin $E$ (IgE) at specified time points (time frame: screening (Day -35), Day 1, Day 112, Day 168, Day 196)

\begin{tabular}{ll}
\hline Starting date & November 2017 \\
\hline Contact information & - \\
\hline Notes & Actual completion date: March 2019 \\
& Expected publica ton date: March 2020 \\
& Publication of study results not required until March 2020 \\
\hline
\end{tabular}

\section{POLYP 2}

\begin{tabular}{ll}
\hline Trial name or title & POLYP 2 (NCT03280537) \\
\hline Methods & Randomised controlled trial \\
\hline Participants & Adults (18 to 75 years) \\
\hline Interventions & Omalizumab \\
\hline
\end{tabular}

Outcomes

Primary outcome measures:

1. Change from baseline in average daily nasal congestion score (NCS) at week 24 (time frame: baseline, week 24)

2. Change from baseline in nasal polyp score (NPS) to week 24 (time frame: baseline, week 24)

Secondary outcome measures:

1. Change from baseline in average daily total nasal symptom score (TNSS) at week 24 (time frame: baseline, week 24)

2. Change from baseline in average daily sense of smell score at week 24 (time frame: baseline, week 24)

3. Change from baseline in average daily posterior rhinorrhoea score at week 24 (time frame: baseline, week 24)

4. Change from baseline in average daily anterior rhinorrhoea score at week 24 (time frame: baseline, week 24)

5. Change from baseline in participant-reported health-related quality of life (HRQL) as assessed by the total Sino-Nasal Outcome Test (SNOT)-22 at week 24 (time frame: baseline, week 24)

6. Change from baseline in sense of smell, as assessed by the University of Pennsylvania Smell Identification Test (UPSIT) at week 24 (time frame: baseline, week 24)

7. Change from baseline in Asthma Quality of Life Questionnaire (AQLQ) of $\geq 0.5$ (in participants with comorbid asthma only) at week 24 (time frame: baseline, week 24)

8. Change from baseline in average daily NCS at week 16 (time frame: baseline, week 16) 
POLYP 2 (Continued)

9. Change from baseline in NPS at week 16 (time frame: baseline, week 16)

10.Percentage of participants with reduction in the need for surgery by week 24 , as defined by a NPS of $\leq 4$ (unilateral score of $\leq 2$ on each Side) and improvement in SNOT-22 score of $\geq 8.9$ (time frame: up to week 24)

11.Percentage of participants requiring of rescue treatment (systemic corticosteroid for $\geq 3$ consecutive days) or having had surgery for nasal polyps through week 24 (time frame: up to week 24)

12.Percentage of participants requiring of rescue treatment (systemic corticosteroid for $\geq 3$ consecutive days) through week 24 (time frame: up to week 24)

13.Percentage of participants having had surgery for nasal polyps through week 24 (time frame: up to week 24)

14.Percentage of participants with adverse events (time frame: up to week 28)

15.Percentage of participants with serious adverse events (time frame: up to week 28)

16.Percentage of participants with adverse events leading to omalizumab/placebo discontinuation (time frame: up to week 28)

17.Percentage of participants with clinically significant change in laboratory values (time frame: up to week 28)

18.Serum concentration of omalizumab at specified time points (time frame: Day 1, Day 112, Day 168, Day 196)

19.Serum concentration of total and free immunoglobulin $\mathrm{E}$ (IgE) at specified time points (time frame: screening (Day -35), Day 1, Day 112, Day 168, Day 196)

\begin{tabular}{ll}
\hline Starting date & November 2019 \\
\hline Contact information & -
\end{tabular}

Notes

Actual completion date: March 2019

Expected publication date: March 2020

Publication of study results not required until March 2020

\section{SYNAPSE}

Trial name or title SYNAPSE (NCT03085797)

Methods Randomised controlled trial

- Participants who have had at least one previous surgery in the previous 10 years for the removal of nasal polyps. Nasal polyp surgery is defined as any procedure involving instruments with resulting incision (cutting open) and removal of polyp tissue from the nasal cavity (polypectomy). For the purpose of inclusion into this study, any procedure involving instrumentation in the nasal cavity resulting in dilatation of the nasal passage such as balloon sinuplasty, insertion of coated stents or direct injection of steroids or other medication without any removal of nasal polyp tissue is not accepted.

- Bilateral nasal polyps as diagnosed by endoscopy or computed tomography (CT) scan. The presence of at least 2 of the following symptoms one of which should be either nasal blockage/obstruction/congestion or nasal discharge (anterior/posterior nasal drip) and either nasal discharge (anterior/posterior nasal drip); facial pain/pressure; reduction or loss of smell for at least 12 weeks prior to screening

- Presence of at least 2 of the following symptoms one of which should be either nasal blockage/obstruction/congestion or nasal discharge (anterior/posterior nasal drip) and either nasal discharge (anterior/posterior nasal drip); facial pain/pressure; reduction or loss of smell for at least 12 weeks prior to screening.

- Severe nasal polyp symptoms defined as an obstruction VAS symptom score of $>5$. 
- Severity consistent with a need for surgery as described by: participants with an overall VAS symptom score $>7$, participants with an endoscopic bilateral nasal polyp score of at least 5 out of a maximum score of 8 (with a minimum score of 2 in each nasal cavity).

Interventions
Mepolizumab injection $100 \mathrm{mg} / \mathrm{mL}$

\section{Outcomes}

\section{Primary outcome measures:}

1. Change from baseline in total endoscopic nasal polyp score at week 52 (time frame: baseline and week 52). Each nostril was assessed for polyps and graded at week 0, 4, 8, 12, 16, 20, 24, 28, 32, 36 and 52 . The grading was based on nasal polyp size and recorded as the sum of the right and left nostril scores. Total score ranges from 0 to 8; higher scores indicate worse status. Individual score ranges from 0 (no polyps) to 4 (large polyps causing almost complete congestion/obstruction of the inferior meatus).

2. Change from baseline in mean nasal obstruction visual analogue scale (VAS) score during the 4 weeks prior to week 52 (time frame: baseline and up to week 52). VAS is an instrument that measures a characteristic or attitude that is believed to range across a continuum of values and cannot easily be directly measured. The participant will be asked to indicate on a VAS ( 0 to 100 units on an electronic device which corresponds to a 0 to 10 score) the severity of 5 nasal polyposis symptoms, one VAS for each symptom (1) nasal obstruction; 2) nasal discharge; 3) mucus in the throat; 4) loss of smell; 5) facial pain) and overall VAS symptoms score. The left hand side of the scale (0) represents "None" and the right hand side of the scale (100) represents "As bad as you can imagine". The VAS score will be collected daily in morning from screening up to week 52.

Secondary outcome measures:

1. Time to first nasal surgery up to week 52 (time frame: up to week 52). Nasal polyp surgery is defined as any procedure involving instruments resulting in incision and removal of tissue (polypectomy) or dilatation of the air passages (e.g. balloon sinuplasty) in the nasal cavity. Time to first nasal surgery up to week 52 will be assessed.

2. Change from baseline in mean overall VAS symptom score during the 4 weeks prior to week 52 (time frame: baseline and up to week 52). The mean VAS score over the last 7 days before Visit 2 (week 0 ) will be used to determine the baseline value. The participant will be asked to indicate on a VAS ( 0 to 100 units on an electronic device which corresponds to 0 to 10 score) the severity of 5 nasal polyposis symptoms, one VAS for each symptom (1) nasal obstruction; 2) nasal discharge; 3) mucus in the throat; 4) loss of smell; 5) facial pain) and overall VAS symptoms score. The left hand side of the scale (0) represents "None" and the right hand side of the scale (100) represents "As bad as you can imagine". The VAS score will be collected daily in morning from screening up to week 52.

3. Change from baseline in Sino-Nasal Outcome Test (SNOT)-22 total score at week 52 (time frame: baseline and week 52). The SNOT-22 is a health-related quality of life questionnaire and has been shown to be a reliable outcome measure for successful septal surgery and in chronic rhinosinusitis management. It is also a tool to evaluate outcomes in nasal polyposis. Participants will be asked to rate the severity of their condition on each of the 22 items over the previous 2 weeks using a 6point rating scale of 0 to 5 including: $0=$ not present $/$ no problem; $1=$ very mild problem; $2=$ mild or slight problem; 3 = moderate problem; 4 = severe problem; 5 = problem as "bad as it can be". The theoretical total score range for the SNOT-22 is 0 to 110 , where lower scores imply less severe symptoms and higher scores represent a worse quality of life. The SNOT-22 questionnaire will be completed by participants at weeks $0,4,8,12,16,20,24,28,32,36$ and 52.

4. Number of $\mathrm{mg}$ per year of prednisolone-equivalent oral corticosteroid dose up to week 52 (time frame: up to week 52). The number of courses of systemic steroids as well as the dose and duration of the courses will be recorded. The dose for a course of oral corticosteroids will be according to the participants SoC for oral corticosteroid use for its nasal polyps condition. A course of systemic corticosteroids is considered continuous if treatment is separated by less than 7 days. Various doses of intravenous and oral steroids will be converted to prednisolone-equivalent oral corticosteroid. 
SYNAPSE (Continued)

Contact information

Notes

Expected study completion date: December 2019

Expected publication: December 2020

GSK intend to make IPD available 6 months after publication of the primary endpoints. Publication not required until December 2020.

CT: computed tomography

INCS: intranasal corticosteroids

IV: intravenous

NBS: nasal blockage score

NCS: nasal congestion score

NP: nasal polyposis

NPS: nasal polyp score

SCS: systemic corticosteroids

SNOT-22: Sino-Nasal Outcome Test-2

TEAE: treatment-emergent adverse event

\section{DATA AND ANALYSES}

\section{Comparison 1. Anti-IL-4Ra mAb (dupilumab) versus placebo (on top of topical steroids)}

\begin{tabular}{|c|c|c|c|c|}
\hline Outcome or subgroup title & $\begin{array}{l}\text { No. of } \\
\text { studies }\end{array}$ & $\begin{array}{l}\text { No. of } \\
\text { partici- } \\
\text { pants }\end{array}$ & Statistical method & Effect size \\
\hline $\begin{array}{l}1 \mathrm{HRQL} \text { - disease-specific (SNOT-22, } \\
0 \text { to } 110 \text {, lower = better) }\end{array}$ & 3 & & Mean Difference (IV, Random, 95\% CI) & Subtotals only \\
\hline $1.1 \mathrm{Up}$ to 24 weeks & 3 & 784 & Mean Difference (IV, Random, 95\% CI) & $-19.61[-22.54,-16.69]$ \\
\hline 1.2 At 52 weeks & 1 & 303 & Mean Difference (IV, Random, 95\% CI) & $-22.38[-27.10,-17.66]$ \\
\hline $\begin{array}{l}2 \text { Disease severity - VAS ( } 0 \text { to } 10 \text {, } \\
\text { lower = better) }\end{array}$ & 3 & 784 & Mean Difference (IV, Random, 95\% CI) & $-3.00[-3.47,-2.53]$ \\
\hline 3 Serious adverse events & 3 & 782 & Risk Ratio (M-H, Fixed, 95\% Cl) & $0.45[0.28,0.75]$ \\
\hline $\begin{array}{l}4 \text { Avoidance of surgery - number of } \\
\text { patients who had surgery as rescue } \\
\text { treatment }\end{array}$ & 2 & 725 & Risk Ratio (M-H, Random, 95\% Cl) & $0.17[0.05,0.52]$ \\
\hline $\begin{array}{l}5 \text { Extent of disease - endoscopy } \\
\text { ('nasal polyps score', } 0 \text { to } 8 \text {, higher = } \\
\text { worse) }\end{array}$ & 3 & & Mean Difference (IV, Random, 95\% CI) & Subtotals only \\
\hline 5.1 Up to 24 weeks & 3 & 784 & Mean Difference (IV, Random, 95\% CI) & $-1.80[-2.25,-1.35]$ \\
\hline 5.2 Up to 52 weeks & 1 & 303 & Mean Difference (IV, Random, 95\% CI) & $-2.34[-2.77,-1.91]$ \\
\hline $\begin{array}{l}6 \text { Extent of disease }- \text { CT scan (Lund } \\
\text { Mackay, } 0 \text { to } 24 \text {, higher = worse) }\end{array}$ & 3 & 784 & Mean Difference (IV, Random, 95\% CI) & $-7.00[-9.61,-4.39]$ \\
\hline
\end{tabular}




\begin{tabular}{llllll}
\hline Outcome or subgroup title & $\begin{array}{l}\text { No. of } \\
\text { studies }\end{array}$ & $\begin{array}{l}\text { No. of } \\
\text { partici- } \\
\text { pants }\end{array}$ & Statistical method & Effect size \\
\hline $\begin{array}{l}7 \mathrm{HRQL} \text { - generic (EQ-5D VAS, 0 to } \\
100, \text { higher = better) }\end{array}$ & 2 & 706 & Mean Difference (IV, Random, 95\% Cl) & $-8.59[-11.86,-5.31]$ \\
\hline $\begin{array}{l}8 \text { Adverse events - nasopharyngitis, } \\
\text { including sore throat (longest avail- } \\
\text { able data) }\end{array}$ & 3 & 783 & Risk Ratio (M-H, Random, 95\% Cl) & $0.95[0.72,1.25]$ \\
\hline
\end{tabular}

Analysis 1.1. Comparison 1 Anti-IL-4Ra mAb (dupilumab) versus placebo (on top of topical steroids), Outcome $1 \mathrm{HRQL}$ - disease-specific (SNOT-22, 0 to 110, lower = better).

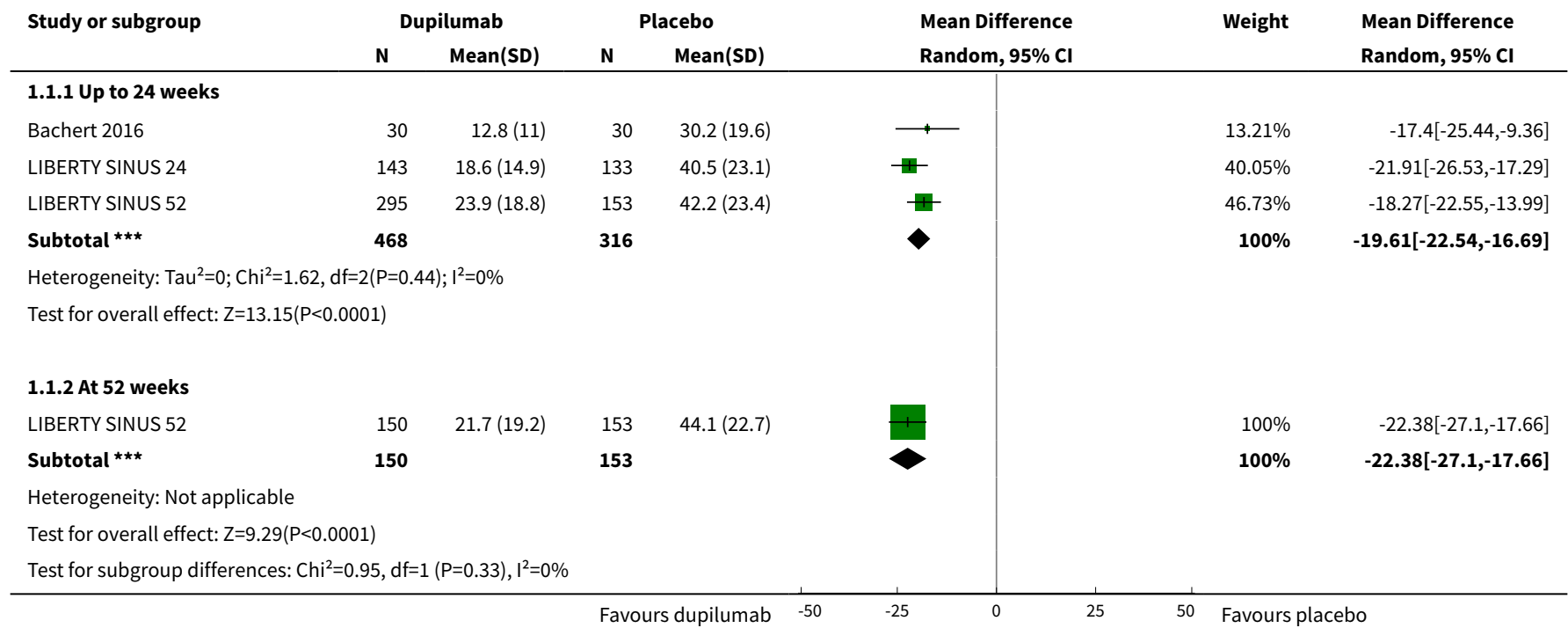

Analysis 1.2. Comparison 1 Anti-IL-4Ra mAb (dupilumab) versus placebo (on top of topical steroids), Outcome 2 Disease severity - VAS (0 to 10, lower = better).

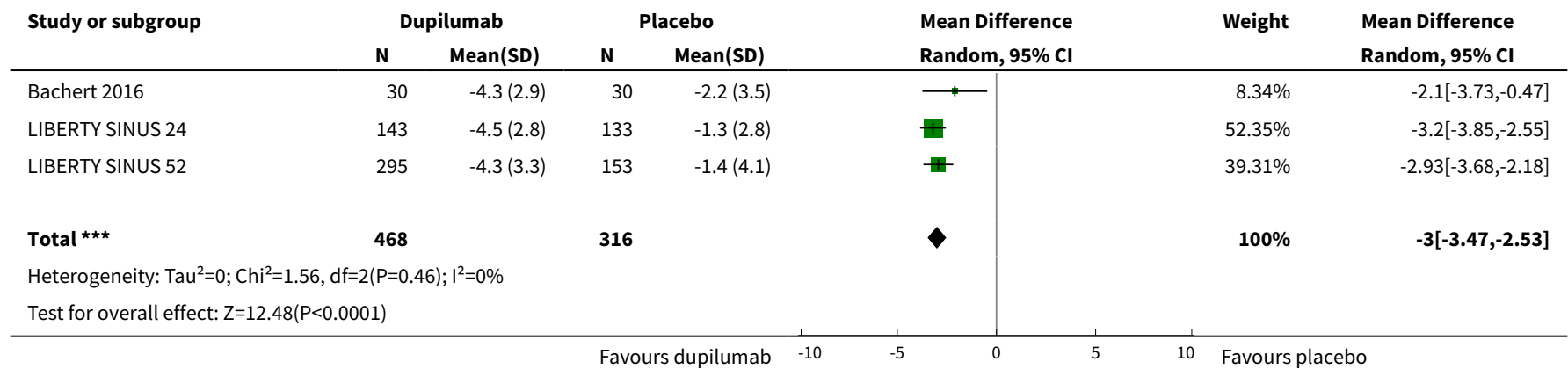


Analysis 1.3. Comparison 1 Anti-IL-4Ra mAb (dupilumab) versus placebo (on top of topical steroids), Outcome 3 Serious adverse events.

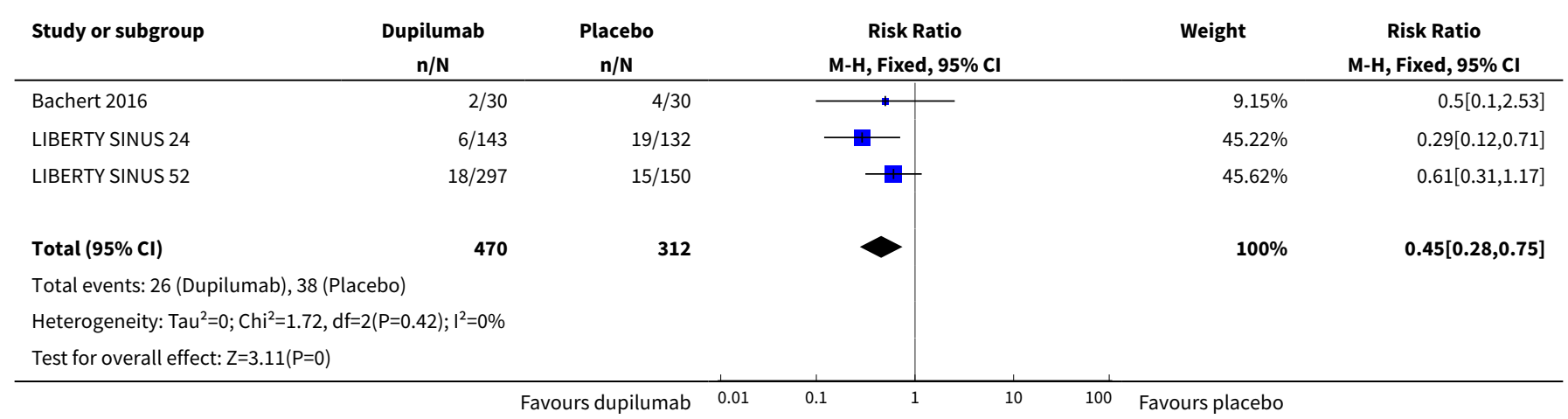

Analysis 1.4. Comparison 1 Anti-IL-4Ra mAb (dupilumab) versus placebo (on top of topical steroids), Outcome 4 Avoidance of surgery - number of patients who had surgery as rescue treatment.

\begin{tabular}{|c|c|c|c|c|c|}
\hline Study or subgroup & $\begin{array}{c}\text { Dupilumab } \\
\mathbf{n} / \mathbf{N} \\
\end{array}$ & $\begin{array}{c}\text { Placebo } \\
\mathbf{n} / \mathbf{N}\end{array}$ & $\begin{array}{c}\text { Risk Ratio } \\
\text { M-H, Random, 95\% Cl }\end{array}$ & Weight & $\begin{array}{c}\text { Risk Ratio } \\
\text { M-H, Random, } 95 \% \text { Cl }\end{array}$ \\
\hline LIBERTY SINUS 24 & $3 / 143$ & $10 / 133$ & + & $55.66 \%$ & $0.28[0.08,0.99]$ \\
\hline LIBERTY SINUS 52 & $2 / 295$ & $12 / 154$ & & $44.34 \%$ & $0.09[0.02,0.38]$ \\
\hline Total $(95 \% \mathrm{Cl})$ & 438 & 287 & & $100 \%$ & $0.17[0.05,0.52]$ \\
\hline \multicolumn{6}{|c|}{ Total events: 5 (Dupilumab), 22 (Placebo) } \\
\hline \multicolumn{6}{|c|}{ Heterogeneity: Tau $^{2}=0.19 ; \mathrm{Chi}^{2}=1.38, \mathrm{df}=1(\mathrm{P}=0.24) ; \mathrm{I}^{2}=27.37 \%$} \\
\hline \multicolumn{6}{|c|}{ Test for overall effect: $Z=3.09(P=0)$} \\
\hline
\end{tabular}

Analysis 1.5. Comparison 1 Anti-IL-4Ra mAb (dupilumab) versus placebo (on top of topical steroids), Outcome 5 Extent of disease - endoscopy ('nasal polyps score', 0 to 8, higher = worse).

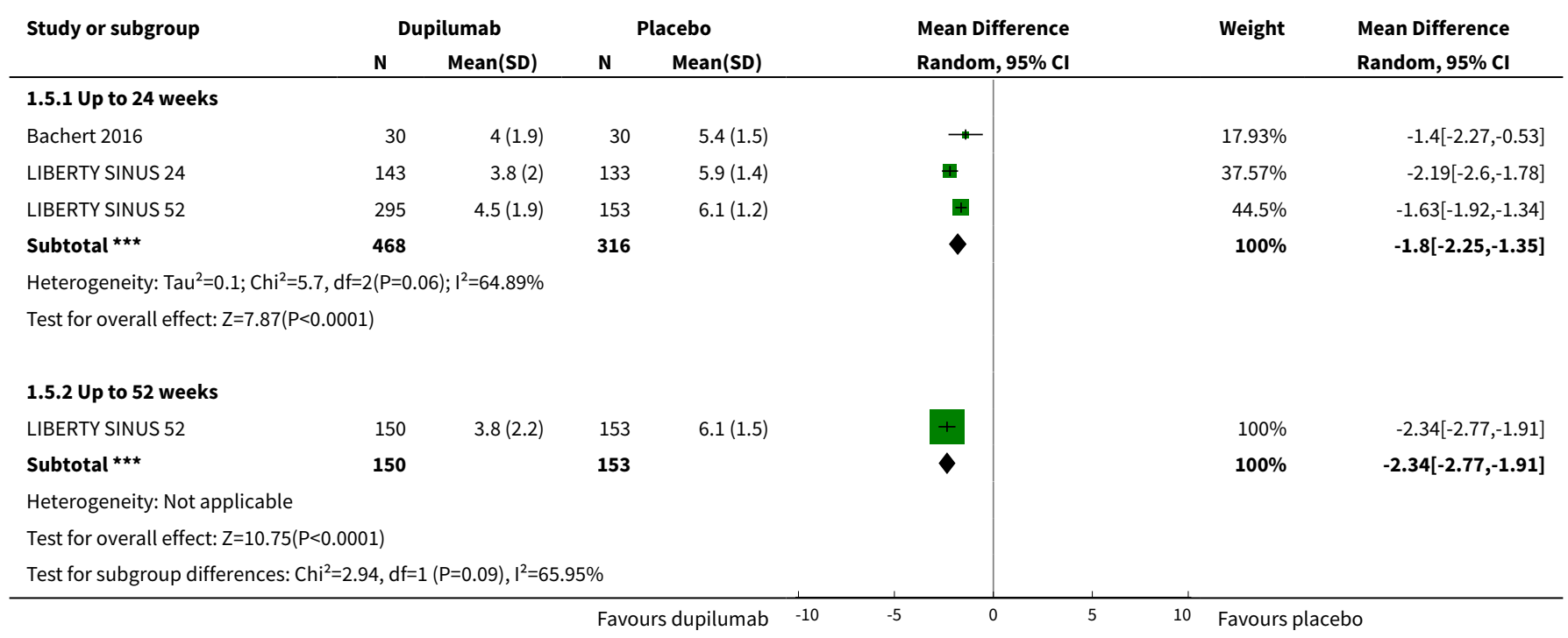




\section{Analysis 1.6. Comparison 1 Anti-IL-4Ra mAb (dupilumab) versus placebo (on top of topical steroids), Outcome 6 Extent of disease - CT scan (Lund Mackay, 0 to 24, higher = worse).}

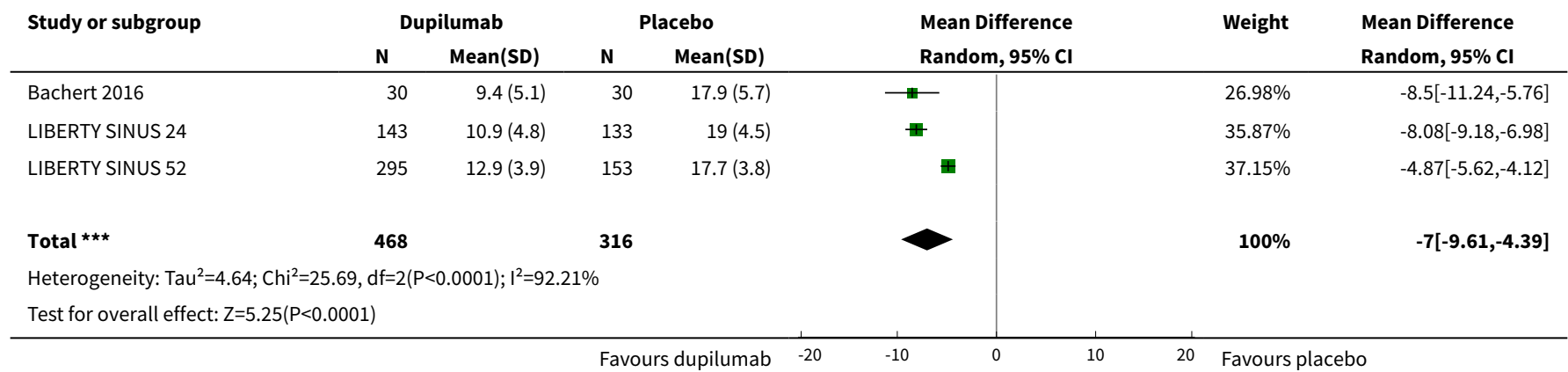

Analysis 1.7. Comparison 1 Anti-IL-4Ra mAb (dupilumab) versus placebo (on top of topical steroids), Outcome $7 \mathrm{HRQL}$ - generic (EQ-5D VAS, 0 to 100, higher = better).

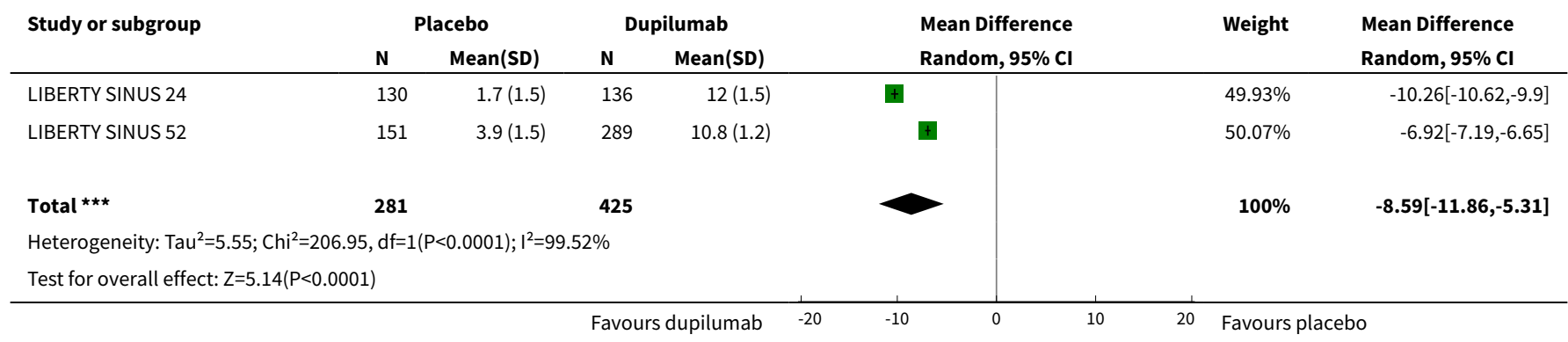

Analysis 1.8. Comparison 1 Anti-IL-4Ra mAb (dupilumab) versus placebo (on top of topical steroids), Outcome 8 Adverse events - nasopharyngitis, including sore throat (longest available data).

\begin{tabular}{|c|c|c|c|c|c|}
\hline Study or subgroup & $\begin{array}{c}\text { Dupilumab } \\
\mathbf{n} / \mathbf{N} \\
\end{array}$ & $\begin{array}{c}\text { Placebo } \\
\mathbf{n} / \mathbf{N}\end{array}$ & $\begin{array}{c}\text { Risk Ratio } \\
\text { M-H, Random, 95\% Cl }\end{array}$ & Weight & $\begin{array}{c}\text { Risk Ratio } \\
\text { M-H, Random, } 95 \% \text { CI }\end{array}$ \\
\hline Bachert 2016 & $14 / 30$ & $10 / 30$ & - & $19.02 \%$ & $1.4[0.74,2.64]$ \\
\hline LIBERTY SINUS 24 & $19 / 143$ & $20 / 133$ & . & $22.61 \%$ & $0.88[0.49,1.58]$ \\
\hline LIBERTY SINUS 52 & $61 / 297$ & $36 / 150$ & & $58.37 \%$ & $0.86[0.6,1.23]$ \\
\hline Total $(95 \% \mathrm{Cl})$ & 470 & 313 & & $100 \%$ & $0.95[0.72,1.25]$ \\
\hline \multicolumn{6}{|c|}{ Heterogeneity: $\mathrm{Tau}^{2}=0 ; \mathrm{Chi}^{2}=1.83, \mathrm{df}=2(\mathrm{P}=0.4) ; \mathrm{I}^{2}=0 \%$} \\
\hline Test for overall effect & & & & & \\
\hline
\end{tabular}


Comparison 2. Anti-IL-5 mAb (mepolizumab) versus placebo (on top of topical steroids)

\begin{tabular}{|c|c|c|c|c|}
\hline Outcome or subgroup title & $\begin{array}{l}\text { No. of } \\
\text { studies }\end{array}$ & $\begin{array}{l}\text { No. of } \\
\text { partici- } \\
\text { pants }\end{array}$ & Statistical method & Effect size \\
\hline $\begin{array}{l}1 \mathrm{HRQL} \text { - SNOT-22 ( } 1 \text { to } 100 \text {, lower = better) up } \\
\text { to } 25 \text { weeks }\end{array}$ & 1 & 105 & $\begin{array}{l}\text { Mean Difference (IV, Random, 95\% } \\
\mathrm{CI})\end{array}$ & $\begin{array}{l}-13.26[-22.08 \\
-4.44]\end{array}$ \\
\hline $\begin{array}{l}2 \text { Disease severity - VAS ( } 0 \text { to } 10 \text {, lower = bet- } \\
\text { ter) }\end{array}$ & 1 & 72 & $\begin{array}{l}\text { Mean Difference (IV, Random, 95\% } \\
\mathrm{CI})\end{array}$ & $-2.03[-3.65,-0.41]$ \\
\hline 3 Severe adverse events & 2 & 135 & Risk Ratio (M-H, Fixed, 95\% Cl) & $1.57[0.07,35.46]$ \\
\hline $\begin{array}{l}4 \text { Avoidance of surgery - patients no longer } \\
\text { meeting criteria for surgery at end of fol- } \\
\text { low-up }\end{array}$ & 2 & 135 & Risk Ratio (M-H, Random, 95\% Cl) & $0.78[0.64,0.94]$ \\
\hline $\begin{array}{l}4.1 \text { Patients still meeting criteria for surgery } \\
\text { at } 24 \text { weeks }\end{array}$ & 1 & 105 & Risk Ratio (M-H, Random, 95\% Cl) & $0.78[0.64,0.95]$ \\
\hline $\begin{array}{l}4.2 \text { Patients requiring 'rescue' surgery during } \\
\text { trial }\end{array}$ & 1 & 30 & Risk Ratio (M-H, Random, 95\% Cl) & $0.67[0.18,2.42]$ \\
\hline 5 Extent of disease - endoscopic score & 2 & 137 & $\begin{array}{l}\text { Mean Difference (IV, Random, 95\% } \\
\mathrm{Cl} \text { ) }\end{array}$ & $-1.23[-1.79,-0.68]$ \\
\hline $\begin{array}{l}6 \mathrm{HRQL} \text { - generic measured using EQ-5D VAS } \\
\text { (range } 0 \text { to } 100 ; 0=\text { worst, } 100=\text { best imagin- } \\
\text { able health state) at week } 25\end{array}$ & 1 & 105 & $\begin{array}{l}\text { Mean Difference (IV, Random, 95\% } \\
\mathrm{CI})\end{array}$ & $5.68[-1.18,12.54]$ \\
\hline $\begin{array}{l}7 \text { Adverse events - nasopharyngitis, including } \\
\text { sore throat }\end{array}$ & 2 & 135 & Risk Ratio (M-H, Random, 95\% Cl) & $0.73[0.36,1.47]$ \\
\hline
\end{tabular}

Analysis 2.1. Comparison 2 Anti-IL-5 mAb (mepolizumab) versus placebo (on top of topical steroids), Outcome $1 \mathrm{HRQL}$ - SNOT-22 (1 to 100, lower = better) up to 25 weeks.

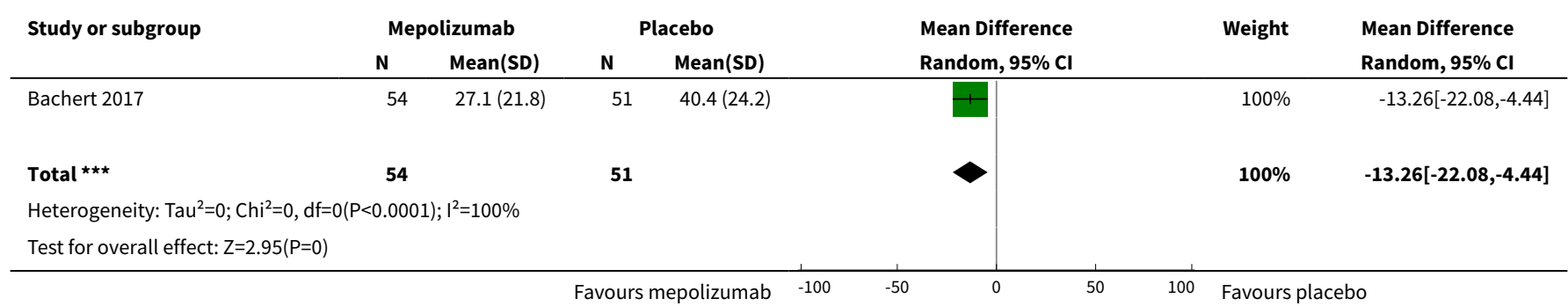


Analysis 2.2. Comparison 2 Anti-IL-5 mAb (mepolizumab) versus placebo (on top of topical steroids), Outcome 2 Disease severity - VAS (0 to 10, lower = better).

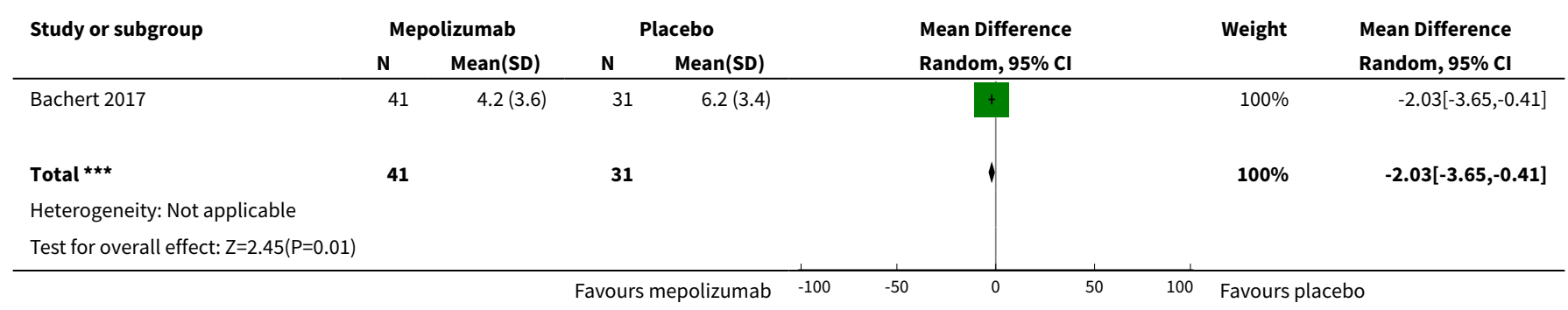

Analysis 2.3. Comparison 2 Anti-IL-5 mAb (mepolizumab) versus placebo (on top of topical steroids), Outcome 3 Severe adverse events.

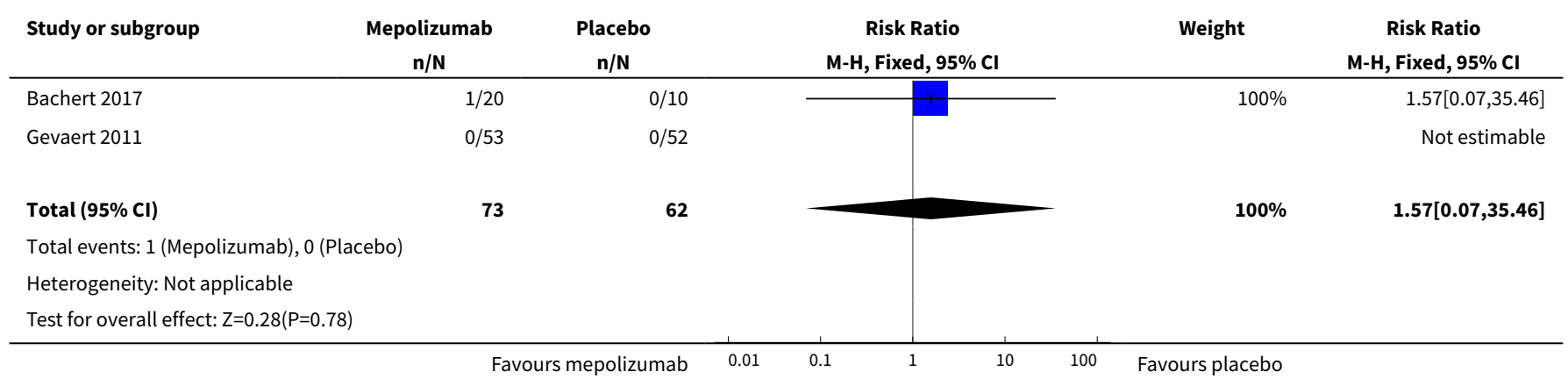

Analysis 2.4. Comparison 2 Anti-IL-5 mAb (mepolizumab) versus placebo (on top of topical steroids), Outcome 4 Avoidance of surgery - patients no longer meeting criteria for surgery at end of follow-up.

\begin{tabular}{|c|c|c|c|c|c|}
\hline Study or subgroup & $\begin{array}{c}\text { Mepolizumab } \\
\mathrm{n} / \mathrm{N}\end{array}$ & $\begin{array}{l}\text { Placebo } \\
\mathrm{n} / \mathrm{N}\end{array}$ & $\begin{array}{c}\text { Risk Ratio } \\
\text { M-H, Random, 95\% Cl }\end{array}$ & Weight & $\begin{array}{c}\text { Risk Ratio } \\
\text { M-H, Random, } 95 \% \mathrm{Cl}\end{array}$ \\
\hline \multicolumn{6}{|c|}{ 2.4.1 Patients still meeting criteria for surgery at 24 weeks } \\
\hline Bachert 2017 & $38 / 54$ & $46 / 51$ & & $97.76 \%$ & $0.78[0.64,0.95]$ \\
\hline Subtotal $(95 \% \mathrm{Cl})$ & 54 & 51 & $\Delta$ & $\mathbf{9 7 . 7 6 \%}$ & $0.78[0.64,0.95]$ \\
\hline \multicolumn{6}{|c|}{ Total events: 38 (Mepolizumab), 46 (Placebo) } \\
\hline \multicolumn{6}{|c|}{ Heterogeneity: Not applicable } \\
\hline \multicolumn{6}{|c|}{ Test for overall effect: $Z=2.49(P=0.01)$} \\
\hline \multicolumn{6}{|c|}{ 2.4.2 Patients requiring 'rescue' surgery during trial } \\
\hline Gevaert 2011 & $4 / 20$ & $3 / 10$ & - & $2.24 \%$ & $0.67[0.18,2.42]$ \\
\hline Subtotal $(95 \% \mathrm{Cl})$ & 20 & 10 & & $2.24 \%$ & $0.67[0.18,2.42]$ \\
\hline \multicolumn{6}{|c|}{ Total events: 4 (Mepolizumab), 3 (Placebo) } \\
\hline \multicolumn{6}{|c|}{ Heterogeneity: Not applicable } \\
\hline \multicolumn{6}{|c|}{ Test for overall effect: $\mathrm{Z}=0.62(\mathrm{P}=0.54)$} \\
\hline Total $(95 \% \mathrm{Cl})$ & 74 & 61 & $\diamond$ & $100 \%$ & $0.78[0.64,0.94]$ \\
\hline \multicolumn{6}{|c|}{ Total events: 42 (Mepolizumab), 49 (Placebo) } \\
\hline \multicolumn{6}{|c|}{ Heterogeneity: $\mathrm{Tau}^{2}=0 ; \mathrm{Chi}^{2}=0.06, \mathrm{df}=1(\mathrm{P}=0.8) ; 1^{2}=0 \%$} \\
\hline \multicolumn{6}{|c|}{ Test for overall effect: $Z=2.56(P=0.01)$} \\
\hline
\end{tabular}




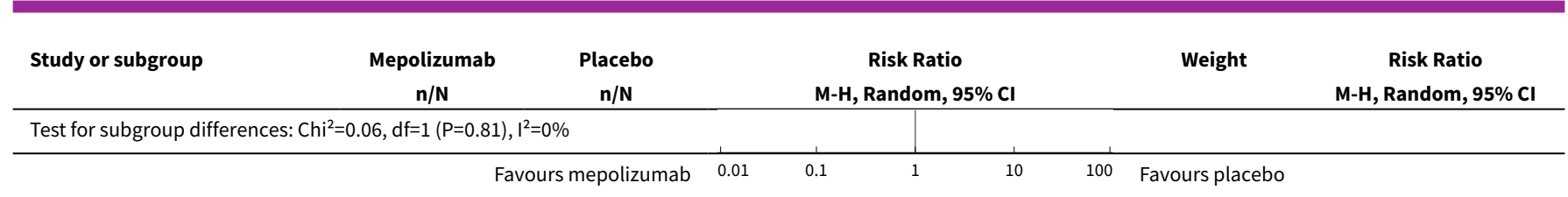

Analysis 2.5. Comparison 2 Anti-IL-5 mAb (mepolizumab) versus placebo (on top of topical steroids), Outcome 5 Extent of disease - endoscopic score.

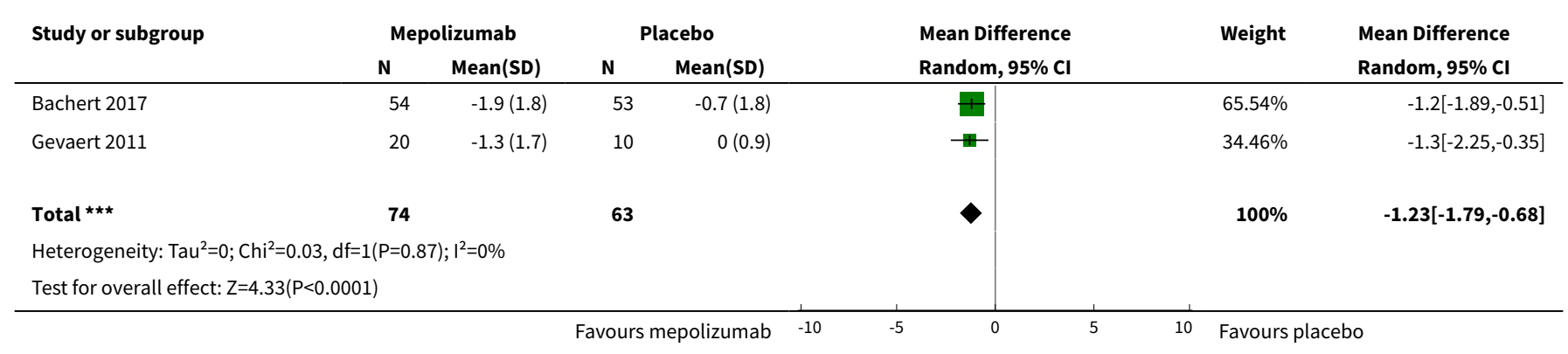

Analysis 2.6. Comparison 2 Anti-IL-5 mAb (mepolizumab) versus placebo (on top of topical steroids), Outcome 6 HRQL - generic measured using EQ-5D VAS (range 0 to 100; 0 = worst, 100 = best imaginable health state) at week 25.

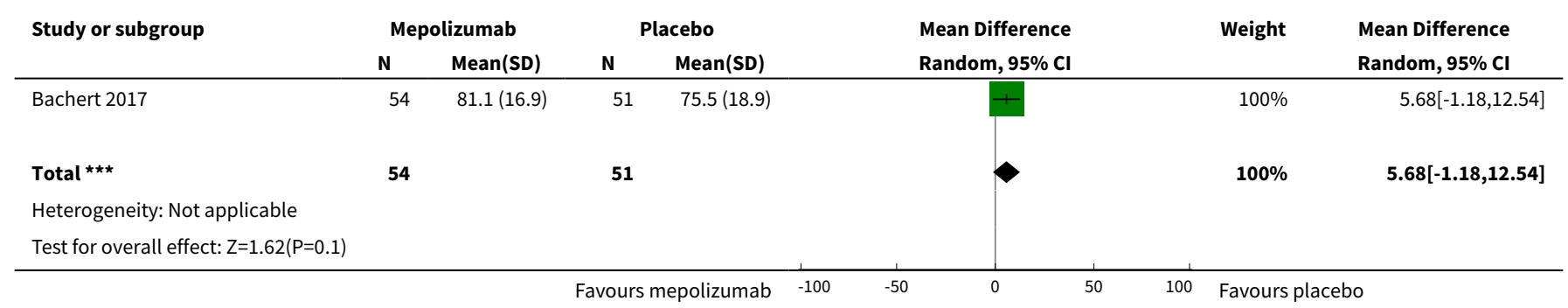

Analysis 2.7. Comparison 2 Anti-IL-5 mAb (mepolizumab) versus placebo (on top of topical steroids), Outcome 7 Adverse events - nasopharyngitis, including sore throat.

\begin{tabular}{|c|c|c|c|c|c|}
\hline Study or subgroup & $\begin{array}{c}\text { Mepolizumab } \\
\mathbf{n} / \mathbf{N} \\
\end{array}$ & $\begin{array}{c}\text { Placebo } \\
\mathbf{n} / \mathbf{N}\end{array}$ & $\begin{array}{c}\text { Risk Ratio } \\
\text { M-H, Random, } 95 \% \mathrm{CI}\end{array}$ & Weight & $\begin{array}{c}\text { Risk Ratio } \\
\text { M-H, Random, } 95 \% \mathrm{CI}\end{array}$ \\
\hline Bachert 2017 & $10 / 53$ & $14 / 52$ & 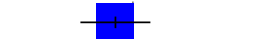 & $94.99 \%$ & $0.7[0.34,1.43]$ \\
\hline Gevaert 2011 & $1 / 20$ & $0 / 10$ & 1 & $5.01 \%$ & $1.57[0.07,35.46]$ \\
\hline Total $(95 \% \mathrm{CI})$ & 73 & 62 & & $100 \%$ & $0.73[0.36,1.47]$ \\
\hline \multicolumn{6}{|c|}{ Total events: 11 (Mepolizumab), 14 (Placebo) } \\
\hline Test for overall effect & & & & & \\
\hline
\end{tabular}


Comparison 3. Anti-IgE mAb (omalizumab) versus placebo (on top of topical steroids)

\begin{tabular}{|c|c|c|c|c|}
\hline Outcome or subgroup title & $\begin{array}{l}\text { No. of } \\
\text { studies }\end{array}$ & $\begin{array}{l}\text { No. of } \\
\text { partici- } \\
\text { pants }\end{array}$ & Statistical method & Effect size \\
\hline 1 Severe adverse events & 3 & 64 & Risk Ratio (M-H, Fixed, 95\% Cl) & $0.0[0.0,0.0]$ \\
\hline 2 Extent of disease - endoscopic score & 2 & 47 & Std. Mean Difference (IV, Random, 95\% CI) & $-1.51[-4.22,1.21]$ \\
\hline $\begin{array}{l}3 \text { Extent of disease - CT scan (lower } \\
\text { score = better) }\end{array}$ & 2 & 47 & Std. Mean Difference (IV, Random, 95\% CI) & $-0.20[-1.55,1.14]$ \\
\hline $\begin{array}{l}4 \text { Adverse events - nasopharyngitis, } \\
\text { including sore throat }\end{array}$ & 3 & 64 & Risk Ratio (M-H, Random, 95\% Cl) & $0.0[0.0,0.0]$ \\
\hline
\end{tabular}

Analysis 3.1. Comparison 3 Anti-IgE mAb (omalizumab) versus placebo (on top of topical steroids), Outcome 1 Severe adverse events.

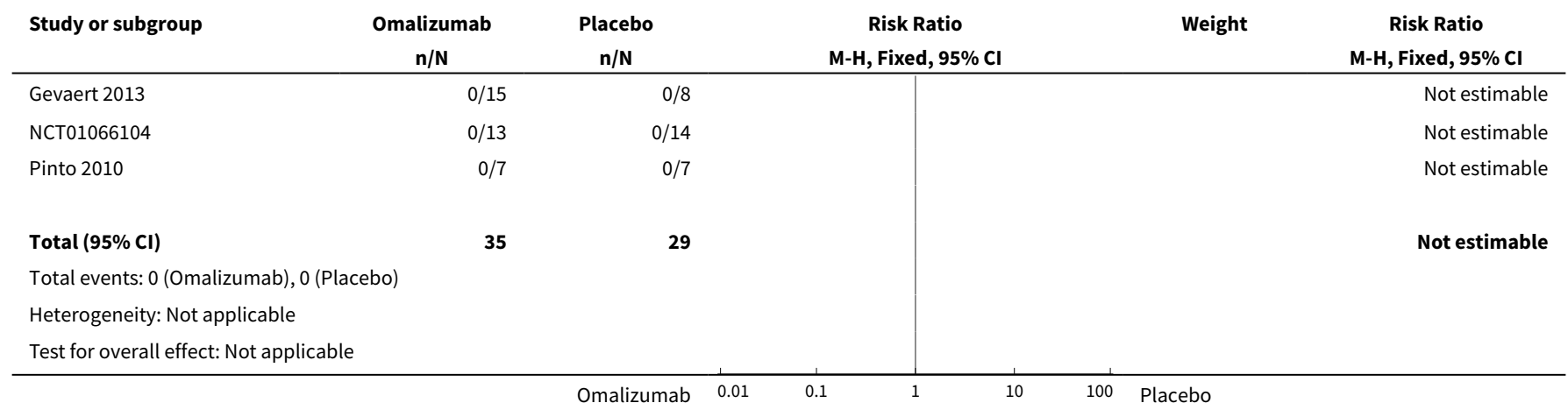

Analysis 3.2. Comparison 3 Anti-IgE mAb (omalizumab) versus placebo (on top of topical steroids), Outcome 2 Extent of disease - endoscopic score.

\begin{tabular}{|c|c|c|c|c|c|c|c|c|}
\hline \multirow[t]{2}{*}{ Study or subgroup } & \multicolumn{2}{|c|}{ Omalizumab } & \multicolumn{2}{|c|}{ Placebo } & \multirow{2}{*}{\multicolumn{2}{|c|}{$\begin{array}{c}\text { Std. Mean Difference } \\
\text { Random, } 95 \% \mathrm{Cl}\end{array}$}} & \multirow[t]{2}{*}{ Weight } & \multirow{2}{*}{$\begin{array}{c}\text { Std. Mean Difference } \\
\text { Random, } 95 \% \mathrm{Cl}\end{array}$} \\
\hline & $\mathbf{N}$ & Mean(SD) & $\mathbf{N}$ & $\operatorname{Mean}(S D)$ & & & & \\
\hline Gevaert 2013 & 15 & $-2.7(1)$ & 8 & $-0.1(0.4)$ & & + & $48.37 \%$ & $-2.94[-4.21,-1.67]$ \\
\hline NCT01066104 & 12 & $-0.3(2.1)$ & 12 & $0.1(2.3)$ & & 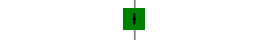 & $51.63 \%$ & $-0.17[-0.97,0.63]$ \\
\hline Total $* \star \star$ & 27 & & 20 & & & 1 & $100 \%$ & $-1.51[-4.22,1.21]$ \\
\hline \multicolumn{9}{|c|}{ Heterogeneity: $\mathrm{Tau}^{2}=3.55 ; \mathrm{Chi}^{2}=13.12, \mathrm{df}=1(\mathrm{P}=0) ; \mathrm{I}^{2}=92.38 \%$} \\
\hline
\end{tabular}


Analysis 3.3. Comparison 3 Anti-IgE mAb (omalizumab) versus placebo (on top of topical steroids), Outcome 3 Extent of disease - CT scan (lower score = better).

\begin{tabular}{|c|c|c|c|c|c|c|c|}
\hline \multirow[t]{2}{*}{ Study or subgroup } & \multicolumn{2}{|c|}{ Omalizumab } & \multicolumn{2}{|c|}{ Placebo } & \multirow{2}{*}{$\begin{array}{c}\text { Std. Mean Difference } \\
\text { Random, } 95 \% \mathrm{Cl}\end{array}$} & \multirow[t]{2}{*}{ Weight } & \multirow{2}{*}{$\begin{array}{c}\text { Std. Mean Difference } \\
\text { Random, } 95 \% \mathrm{Cl}\end{array}$} \\
\hline & $\mathbf{N}$ & Mean(SD) & $\mathbf{N}$ & Mean(SD) & & & \\
\hline Gevaert 2013 & 15 & $13.6(5)$ & 8 & $18.3(5)$ & + & $48.92 \%$ & $-0.91[-1.81,-0]$ \\
\hline NCT01066104 & 12 & $4.2(25.6)$ & 12 & $-8.9(28.2)$ & + & $51.08 \%$ & $0.47[-0.34,1.28]$ \\
\hline 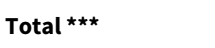 & 27 & & 20 & & 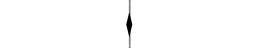 & $100 \%$ & $-0.2[-1.55,1.14]$ \\
\hline \multicolumn{8}{|c|}{ Heterogeneity: $\mathrm{Tau}^{2}=0.75 ; \mathrm{Chi}^{2}=4.91, \mathrm{df}=1(\mathrm{P}=0.03) ; \mathrm{I}^{2}=79.64 \%$} \\
\hline
\end{tabular}

Analysis 3.4. Comparison 3 Anti-IgE mAb (omalizumab) versus placebo (on top of topical steroids), Outcome 4 Adverse events - nasopharyngitis, including sore throat.

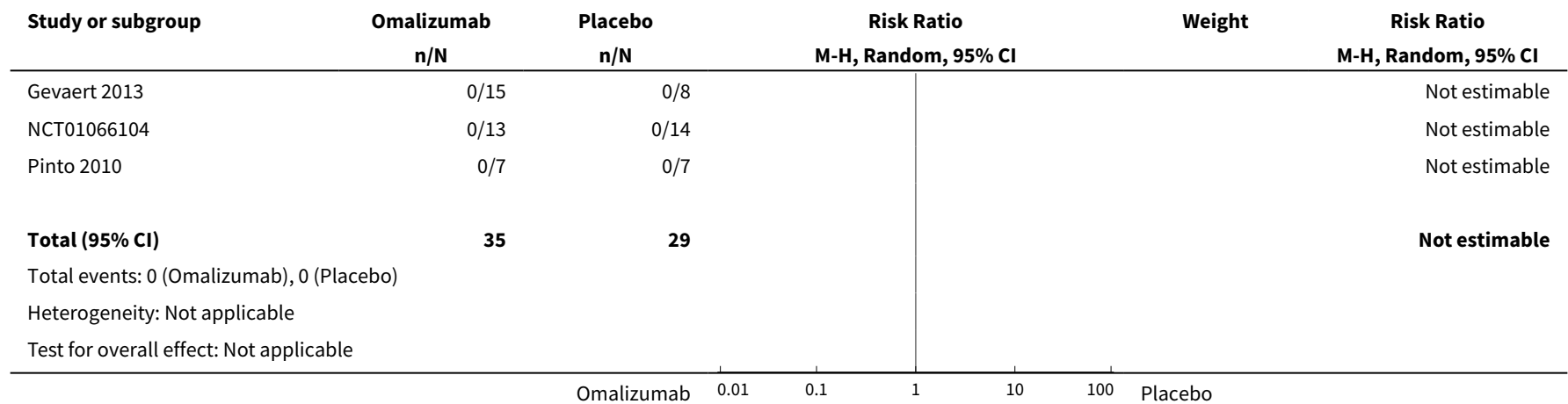

\section{ADDITIONAL TABLES}


Table 1. Summary of characteristics of included studies

\begin{tabular}{|c|c|c|c|c|c|c|c|c|}
\hline & $\begin{array}{l}\text { SINUS } 24 \\
(n=276)\end{array}$ & $\begin{array}{l}\text { SINUS } 52 \\
(n=448)\end{array}$ & $\begin{array}{l}\text { Bachert } \\
2016 \\
(n=60)\end{array}$ & $\begin{array}{l}\text { Bachert } 2017 \\
(n=107)\end{array}$ & $\begin{array}{l}\text { Gevaert } 2011 \\
(n=30)\end{array}$ & $\begin{array}{l}\text { Pinto } 2010 \\
(n=14)\end{array}$ & $\begin{array}{l}\text { Gevaert } 2013 \\
(n=24)\end{array}$ & $\begin{array}{l}\text { NCT01066104 } \\
(n=27)\end{array}$ \\
\hline $\begin{array}{l}\text { Popula- } \\
\text { tion }\end{array}$ & $\begin{array}{l}\text { Bilateral nasal } \\
\text { polyps (mean } \\
5.75 \text { points) } \\
\text { with symptoms } \\
\text { of chronic rhi- } \\
\text { nosinusitis de- } \\
\text { spite intranasal } \\
\text { steroids }\end{array}$ & $\begin{array}{l}\text { Bilateral nasal } \\
\text { polyps (mean } 6.10 \\
\text { points) with symp- } \\
\text { toms of chronic rhi- } \\
\text { nosinusitis despite } \\
\text { intranasal steroids }\end{array}$ & $\begin{array}{l}\text { Chronic } \\
\text { sinusitis } \\
\text { with nasal } \\
\text { polyps } \\
\text { (mean } 5.8 \\
\text { points) }\end{array}$ & $\begin{array}{l}\text { Severe, recur- } \\
\text { rent bilateral } \\
\text { nasal polypo- } \\
\text { sis requiring } \\
\text { surgery (worst } \\
\text { affected nos- } \\
\text { tril } \geq 3 \text { (on 4- } \\
\text { point scale), } \\
\text { and symptoms } \\
\text { score }>7 \text { on } \\
10 \mathrm{~cm} \text { VAS de- } \\
\text { spite intranasal } \\
\text { steroids and/ } \\
\text { or previous oral } \\
\text { corticosteroids } \\
\text { Mean bilateral } \\
\text { polyp score } 6.29\end{array}$ & $\begin{array}{l}\text { Chronic rhinos- } \\
\text { inusitis with se- } \\
\text { vere primary } \\
\text { polyps (grade } \\
3 \text { to 4) or recur- } \\
\text { rent polyps (any } \\
\text { grade) } \\
\text { Failure of stan- } \\
\text { dard care for } \\
\text { chronic rhinosi- } \\
\text { nusitis }\end{array}$ & $\begin{array}{l}\text { Chronic rhinosinusi- } \\
\text { tis } \\
\text { Polyps status: } 7 / 7 \\
\text { in omalizumab and } \\
5 / 7 \text { in placebo had } \\
\text { nasal polyposis }\end{array}$ & $\begin{array}{l}\text { Chronic rhinosi- } \\
\text { nusitis with nasal } \\
\text { polyps } \\
\text { Polyps status: TPS } \\
\text { (total nasal endo- } \\
\text { scopic polyp score), } \\
\text { median (IQR): } 6 \text { (4 } \\
\text { to } 6 \text { ); } 6 \text { (6 to } 8 \text { ) }\end{array}$ & $\begin{array}{l}\text { Chronic rhinos- } \\
\text { inusitis with } \\
\text { nasal polyps } \\
\text { Inclusion crite- } \\
\text { ria state min- } \\
\text { imum polyp } \\
\text { score of } 4\end{array}$ \\
\hline $\begin{array}{l}\text { Comor- } \\
\text { bidity }\end{array}$ & Asthma 58\% & Asthma $60 \%$ & $\begin{array}{l}\text { Asthma } \\
58 \%\end{array}$ & Asthma 78\% & Asthma 43\% & $\begin{array}{l}\text { Inhaled asthma } \\
\text { therapy taken by } \\
72 \%(5 / 7) \text { in omal- } \\
\text { izumab group and } \\
43 \%(3 / 7) \text { in place- } \\
\text { bo group }\end{array}$ & Asthma (100\%) & No information \\
\hline $\begin{array}{l}\text { Eligi- } \\
\text { ble for } \\
\text { surgery? }\end{array}$ & No information & No information & $\begin{array}{l}\text { No infor- } \\
\text { mation }\end{array}$ & Yes $^{a}$ & No information & $\begin{array}{l}100 \% \text { had under- } \\
\text { gone endoscopic } \\
\text { sinus surgery, but } \\
\text { no information on } \\
\text { eligibility for more } \\
\text { surgery }\end{array}$ & No information & No information \\
\hline $\begin{array}{l}\text { Interven- } \\
\text { tion }\end{array}$ & $\begin{array}{l}\text { Dupilumab } 300 \\
\text { mg subcuta- } \\
\text { neously every } 2 \\
\text { weeks }\end{array}$ & $\begin{array}{l}\text { a) Dupilumab } 300 \\
\text { mg subcutaneously } \\
\text { every } 2 \text { weeks for } 24 \\
\text { weeks, followed by } \\
\text { every } 4 \text { weeks until } \\
52 \text { weeks }\end{array}$ & $\begin{array}{l}\text { Dupilum- } \\
\text { ab } 600 \\
\text { mg load- } \\
\text { ing dose } \\
\text { subcuta- } \\
\text { neously, } \\
\text { followed }\end{array}$ & $\begin{array}{l}\text { Mepolizumab } \\
750 \text { mg intra- } \\
\text { venously every } \\
4 \text { weeks }\end{array}$ & $\begin{array}{l}\text { Mepolizumab } \\
750 \text { mg intra- } \\
\text { venously every } \\
4 \text { weeks }\end{array}$ & $\begin{array}{l}\text { Omalizumab sub- } \\
\text { cutaneously, once } \\
\text { or twice monthly } \\
\text { (dose dependent on } \\
\text { participant weight } \\
\text { and serum IgE lev- } \\
\text { el), for } 6 \text { months }\end{array}$ & $\begin{array}{l}\text { Omalizumab sub- } \\
\text { cutaneously every } 2 \\
\text { weeks ( } 8 \text { injections } \\
\text { in total) or every } \\
\text { month ( } 4 \text { injections } \\
\text { in total), based on } \\
\text { total serum IgE }\end{array}$ & $\begin{array}{l}\text { Omalizumab } \\
\text { subcutaneous- } \\
\text { ly, every } 2 \text { to } 4 \\
\text { weeks depend- } \\
\text { ing on baseline } \\
\text { serum total IgE }\end{array}$ \\
\hline
\end{tabular}




\begin{tabular}{|c|c|c|c|c|c|c|c|c|}
\hline & & $\begin{array}{l}\text { b) Dupilumab } 300 \\
\text { mg subcutaneously } \\
\text { every } 2 \text { weeks for } 52 \\
\text { weeks in total }\end{array}$ & $\begin{array}{l}\text { by } 300 \\
\text { mg every } \\
\text { week }\end{array}$ & & & & $\begin{array}{l}\text { levels and body } \\
\text { weight, with a max- } \\
\text { imum dose of } 375 \\
\text { mg }\end{array}$ & $\begin{array}{l}\text { level and body } \\
\text { weight }\end{array}$ \\
\hline $\begin{array}{l}\text { Compari- } \\
\text { son }\end{array}$ & $\begin{array}{l}\text { Placebo subcu- } \\
\text { taneously every } \\
2 \text { weeks }\end{array}$ & $\begin{array}{l}\text { Placebo subcuta- } \\
\text { neously every } 2 \\
\text { weeks }\end{array}$ & $\begin{array}{l}\text { Placebo } \\
\text { subcuta- } \\
\text { neous- } \\
\text { ly every } \\
\text { week }\end{array}$ & $\begin{array}{l}\text { Intravenous } \\
\text { placebo every } 4 \\
\text { weeks }\end{array}$ & $\begin{array}{l}\text { Intravenous } \\
\text { placebo every } 4 \\
\text { weeks }\end{array}$ & $\begin{array}{l}\text { Placebo injection, } \\
\text { same dose and fre- } \\
\text { quency }\end{array}$ & $\begin{array}{l}\text { Placebo injection, } \\
\text { same dose and fre- } \\
\text { quency }\end{array}$ & $\begin{array}{l}\text { Stated as "Xo- } \\
\text { lair placebo } \\
150-375 \mathrm{mg} \\
\text { depending on } \\
\text { baseline serum } \\
\text { total IgE lev- } \\
\text { el and body } \\
\text { weight" }\end{array}$ \\
\hline $\begin{array}{l}\text { Treat- } \\
\text { ment } \\
\text { length }\end{array}$ & 24 weeks & 52 weeks & 15 weeks & 24 weeks & $\begin{array}{l}8 \text { weeks ( } 2 \text { dos- } \\
\text { es) }\end{array}$ & 26 weeks & 16 weeks & 22 weeks \\
\hline $\begin{array}{l}\text { Follow-up } \\
\text { length } \\
\text { (total } \\
\text { treatment } \\
\text { and fol- } \\
\text { low-up } \\
\text { period) }\end{array}$ & 24 weeks & $\begin{array}{l}24 \text { weeks and } 52 \\
\text { weeks }\end{array}$ & 16 weeks & 25 weeks & $\begin{array}{l}48 \text { weeks (most } \\
\text { outcomes as- } \\
\text { sessed after } 8 \\
\text { weeks' treat- } \\
\text { ment) }\end{array}$ & 26 weeks & $\begin{array}{l}20 \text { weeks (out- } \\
\text { comes assessed af- } \\
\text { ter } 16 \text { weeks' treat- } \\
\text { ment) }\end{array}$ & 22 weeks \\
\hline $\begin{array}{l}\text { Specific } \\
\text { HRQL }\end{array}$ & $\begin{array}{l}\text { Measured and } \\
\text { reported } b\end{array}$ & $\begin{array}{l}\text { Measured and re- } \\
\text { ported } b\end{array}$ & $\begin{array}{l}\text { Measured } \\
\text { and re- } \\
\text { ported } b\end{array}$ & $\begin{array}{l}\text { Measured and } \\
\text { reported } b\end{array}$ & Not measured & $\begin{array}{l}\text { Measured and re- } \\
\text { ported } b\end{array}$ & $\begin{array}{l}\text { Measured and re- } \\
\text { ported } c\end{array}$ & Not measured \\
\hline \multirow[t]{2}{*}{$\begin{array}{l}\text { Disease } \\
\text { severity } \\
\text { (overall) }\end{array}$} & \multirow[t]{2}{*}{$\begin{array}{l}\text { Measured and } \\
\text { reported d,e }\end{array}$} & \multirow[t]{2}{*}{$\begin{array}{l}\text { Measured and re- } \\
\text { portedd,e }\end{array}$} & \multirow[t]{2}{*}{$\begin{array}{l}\text { Measured } \\
\text { and re- } \\
\text { portedd,j }\end{array}$} & \multirow[t]{2}{*}{$\begin{array}{l}\text { Measured and } \\
\text { reported } d\end{array}$} & $\begin{array}{l}\text { No global ques- } \\
\text { tionnaire re- } \\
\text { ported }\end{array}$ & $\begin{array}{l}\text { No global question- } \\
\text { naire reported }\end{array}$ & $\begin{array}{l}\text { No global question- } \\
\text { naire reported }\end{array}$ & $\begin{array}{l}\text { No global ques- } \\
\text { tionnaire re- } \\
\text { ported }\end{array}$ \\
\hline & & & & & $\begin{array}{l}\text { Specific symp- } \\
\text { toms measured } \\
\text { and reported } f\end{array}$ & $\begin{array}{l}\text { measured and re- } \\
\text { portedg,h }\end{array}$ & $\begin{array}{l}\text { measured and re- } \\
\text { ported }^{i}\end{array}$ & $\begin{array}{l}\text { Measured but } \\
\text { not reportedk }\end{array}$ \\
\hline $\begin{array}{l}\text { Severe } \\
\text { adverse } \\
\text { event }\end{array}$ & $\begin{array}{l}\text { Measured and } \\
\text { reported }\end{array}$ & $\begin{array}{l}\text { Measured and re- } \\
\text { ported }\end{array}$ & $\begin{array}{l}\text { Measured } \\
\text { and re- } \\
\text { ported }\end{array}$ & $\begin{array}{l}\text { Measured and } \\
\text { reported }\end{array}$ & $\begin{array}{l}\text { Measured and } \\
\text { reported }\end{array}$ & $\begin{array}{l}\text { Measured and re- } \\
\text { ported }\end{array}$ & Not measured & $\begin{array}{l}\text { Measured and } \\
\text { reported }\end{array}$ \\
\hline
\end{tabular}




\begin{tabular}{|c|c|c|c|c|c|c|c|c|}
\hline $\begin{array}{l}\text { Avoid- } \\
\text { ance of } \\
\text { Surgery }\end{array}$ & $\begin{array}{l}\text { Measured and } \\
\text { reportedl,m }\end{array}$ & $\begin{array}{l}\text { Measured and re- } \\
\text { portedl,n }\end{array}$ & $\begin{array}{l}\text { Not mea- } \\
\text { sured }\end{array}$ & $\begin{array}{l}\text { Measured and } \\
\text { reported }{ }^{\circ}\end{array}$ & Not measured & Not measured & Not measured & Not measured \\
\hline CT scan & $\begin{array}{l}\text { Measured and } \\
\text { reported } p\end{array}$ & $\begin{array}{l}\text { Measured and re- } \\
\text { ported } p\end{array}$ & $\begin{array}{l}\text { Measured } \\
\text { and re- } \\
\text { ported } p\end{array}$ & Not measured & $\begin{array}{l}\text { Measured and } \\
\text { reported } q\end{array}$ & $\begin{array}{l}\text { Measured and re- } \\
\text { ported } r\end{array}$ & $\begin{array}{l}\text { Measured and re- } \\
\text { ported } p\end{array}$ & $\begin{array}{l}\text { Measured and } \\
\text { reporteds }^{s}\end{array}$ \\
\hline $\begin{array}{l}\text { Polyps } \\
\text { score }\end{array}$ & $\begin{array}{l}\text { Measured and } \\
\text { reported } t\end{array}$ & $\begin{array}{l}\text { Measured and re- } \\
\text { ported } t\end{array}$ & $\begin{array}{l}\text { Measured } \\
\text { and re- } \\
\text { ported } t\end{array}$ & $\begin{array}{l}\text { Measured and } \\
\text { reported } u\end{array}$ & $\begin{array}{l}\text { Measured and } \\
\text { reported } t\end{array}$ & $\begin{array}{l}\text { Measured and re- } \\
\text { ported } v\end{array}$ & $\begin{array}{l}\text { Measured and re- } \\
\text { ported } t\end{array}$ & $\begin{array}{l}\text { Measured and } \\
\text { reported } t\end{array}$ \\
\hline $\begin{array}{l}\text { Generic } \\
\text { HRQL }\end{array}$ & $\begin{array}{l}\text { Measured and } \\
\text { reportedw,m }\end{array}$ & $\begin{array}{l}\text { Measured and re- } \\
\text { ported } \mathrm{w}, \mathrm{m}\end{array}$ & $\begin{array}{l}\text { Not mea- } \\
\text { sured }\end{array}$ & $\begin{array}{l}\text { Measured and } \\
\text { reported } w, x\end{array}$ & Not measured & $\begin{array}{l}\text { Measured and re- } \\
\text { ported } y\end{array}$ & $\begin{array}{l}\text { Measured and re- } \\
\text { ported } y\end{array}$ & Not measured \\
\hline $\begin{array}{l}\text { Na- } \\
\text { sopharyn- } \\
\text { gitis }\end{array}$ & $\begin{array}{l}\text { Measured and } \\
\text { reported }\end{array}$ & $\begin{array}{l}\text { Measured and re- } \\
\text { ported }\end{array}$ & $\begin{array}{l}\text { Measured } \\
\text { and re- } \\
\text { ported }\end{array}$ & $\begin{array}{l}\text { Measured and } \\
\text { reported }\end{array}$ & $\begin{array}{l}\text { Measured and } \\
\text { reported }\end{array}$ & Not measured $z$ & Not measured & Not measured \\
\hline $\begin{array}{l}\text { Main data } \\
\text { source }\end{array}$ & $\begin{array}{l}\text { Publications; } \\
\text { generic health- } \\
\text { related qual- } \\
\text { ity of life and } \\
\text { avoidance of } \\
\text { surgery data } \\
\text { from trial reg- } \\
\text { istry only }\end{array}$ & $\begin{array}{l}\text { Publications; gener- } \\
\text { ic health-relat- } \\
\text { ed quality of life } \\
\text { and avoidance of } \\
\text { surgery data from } \\
\text { trial registry only }\end{array}$ & $\begin{array}{l}\text { Publica- } \\
\text { tions }\end{array}$ & Publications & Publications & Publication & Publication & $\begin{array}{l}\text { NCT record (no } \\
\text { publications) }\end{array}$ \\
\hline
\end{tabular}

aWorst affected nostril $\geq 3$ (on a 4-point scale), and symptoms score $>7$ on $10 \mathrm{~cm}$ VAS despite intranasal steroids and/or previous oral corticosteroids.

bSNOT-22, scale 0 to 110 , higher $=$ worse, minimal clinically important difference (MID) $\geq 8.9$ points.

CRSOM-31; AQLQ.

$d$ Visual analogue scale for rhinosinusitis: "how troublesome are your symptoms?", scale 0 to $10 \mathrm{~cm}$, higher = worse.

eTotal symptom severity score (including nasal congestion, rhinorrhoea and sense of smell, each rated between 0 and 3 ), total scale 0 to 9 , higher $=$ worse

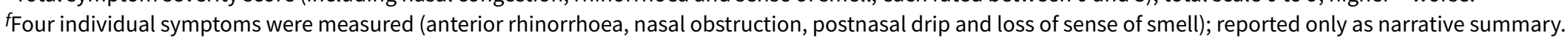

$g$ Total nasal symptom score (TNSS): nasal obstruction, nasal discharge, facial pain and hyposmia) each recorded on a 4 -point scale $(0=$ none, $1=$ mild, $2=$ moderate, $3=$ severe); total scores summed.

hOnly reported as 'no significant difference' - no data presented.

'Disease severity symptom score: nasal and asthma symptoms (patient-reported, daily 'absent, mild, moderate or severe' (scores $0,1,2,3)$.

jSeverity scores for individual symptoms (nasal congestion, anterior and posterior rhinorrhoea, loss in sense of smell, nocturnal awakenings), range 0 to 3 , higher $=$ worse.

kNCT record states that a total symptom score (TSS) and chronic rhinosinusitis facial pain/headache questionnaire were recorded daily; no outcome data presented in NCT record. INumber of participants requiring rescue with nasal polyp surgery - no definition for eligibility provided.

moutcome reported, but specific data only reported in trial registry (publication includes pooled data with SINUS 52 only). 
nOutcome measured but not reported (pooled data with SINUS 24 only, specific data for this trial not reported on trial registry or publication).

oAt study endpoint, participants with a nasal polyp score of $\geq 3$ were deemed as continuing to need surgery (regardless of VAS score). In addition, participants with a nasal polyp score of 2 , who had a VAS score of $>7$ were also viewed as requiring surgery.

pLund Mackay CT score, range 0 to 24, higher $=$ worse.

qPublication reports proportion of participants who showed improvement in CT score during the study. Shown separately for three independent raters, with no summary measure reported.

rMucosal thickness on CT scan.

${ }^{\mathrm{S}} \mathrm{CT}$ scan scored using the Zinreich modification of the Lund Mackay scoring system.

${ }^{t_{B}}$ ilateral "endoscopic nasal polyps score" (NPS) or total polyps score (TPS), range 0 to 8 , higher $=$ worse.

"Improvement by at least one point in endoscopic nasal polyp score.

vNasal endoscopy score (0 to 4). Unclear which scoring system used.

WEQ-5D visual analogue scale, range 0 to 100 (100 = best imaginable).

${ }^{x} \mathrm{EQ}-5 \mathrm{D}$ index score, range 0 to 1 , higher $=$ better.

$y \mathrm{SF}-36$.

zOutcome not specifically mentioned, paper just states "No side effects or adverse events occurred during the study".

\section{Table 2. Eligibility for surgery}

\section{Study name Study}

Eligibility for surgery: defined at

Eligibility criteria for surgery: as recorded in results

randomisation?

Yes

No

De- Yes
scrip-
tion of
how
deci-
sions
were
made
to car-
ry out/
offer
surgery

\section{Completed (included) studies}

\begin{tabular}{|c|c|c|c|c|c|}
\hline $\begin{array}{l}\text { SINUS } 52 \\
\text { (NCT02898454) }\end{array}$ & $\begin{array}{l}\text { EUC- } \\
\text { TR2015-001314-10-ES } \\
2016\end{array}$ & $x$ & $\begin{array}{l}\text { Not } \\
\text { men- } \\
\text { tioned }\end{array}$ & $x$ & $\begin{array}{l}\text { Criteria not defined but one outcome was "Proportion of } \\
\text { patients during study treatment receiving oral corticos- } \\
\text { teroid (OCS) for NP and/or planned to under surgery for nasal } \\
\text { polyps" }\end{array}$ \\
\hline $\begin{array}{l}\text { SINUS } 24 \\
\text { (NCT02898454) }\end{array}$ & $\begin{array}{l}\text { Bachert } 2019 \\
\text { NCT02898454 }\end{array}$ & $x$ & $\begin{array}{l}\text { Not } \\
\text { men- } \\
\text { tioned }\end{array}$ & & $\begin{array}{l}\text { Offered when there was worsening of signs and/or symptoms } \\
\text { during the study }\end{array}$ \\
\hline
\end{tabular}




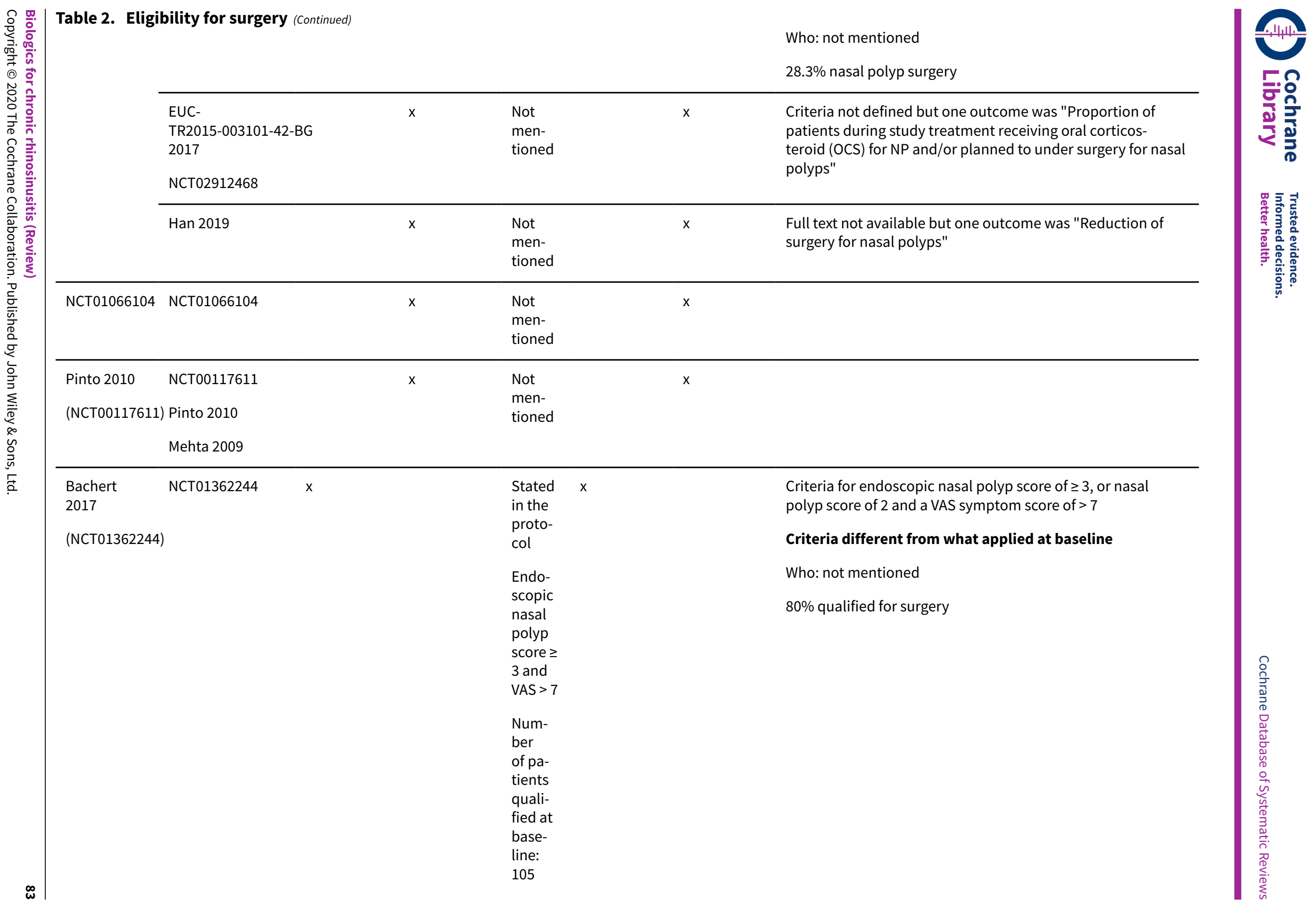




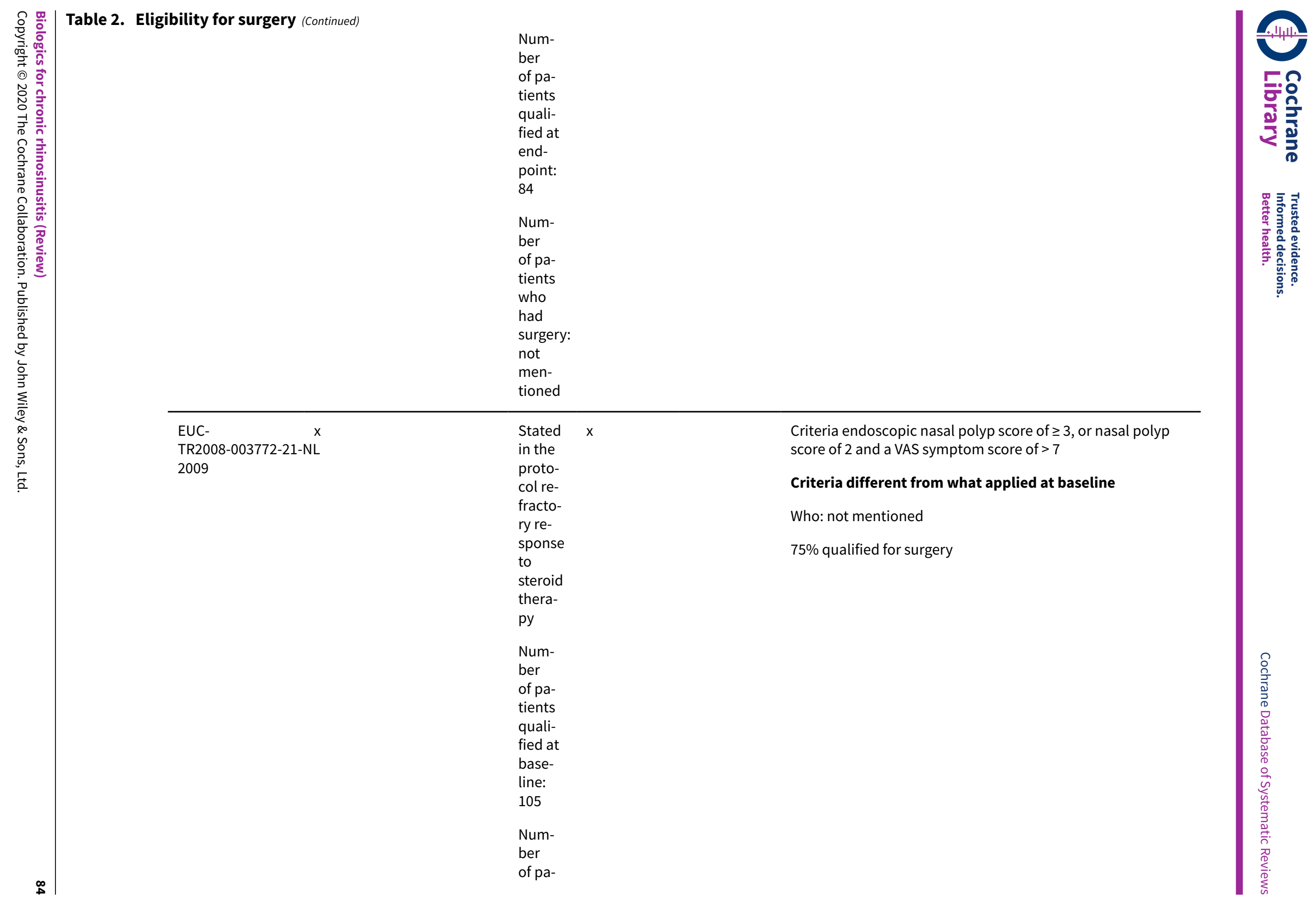




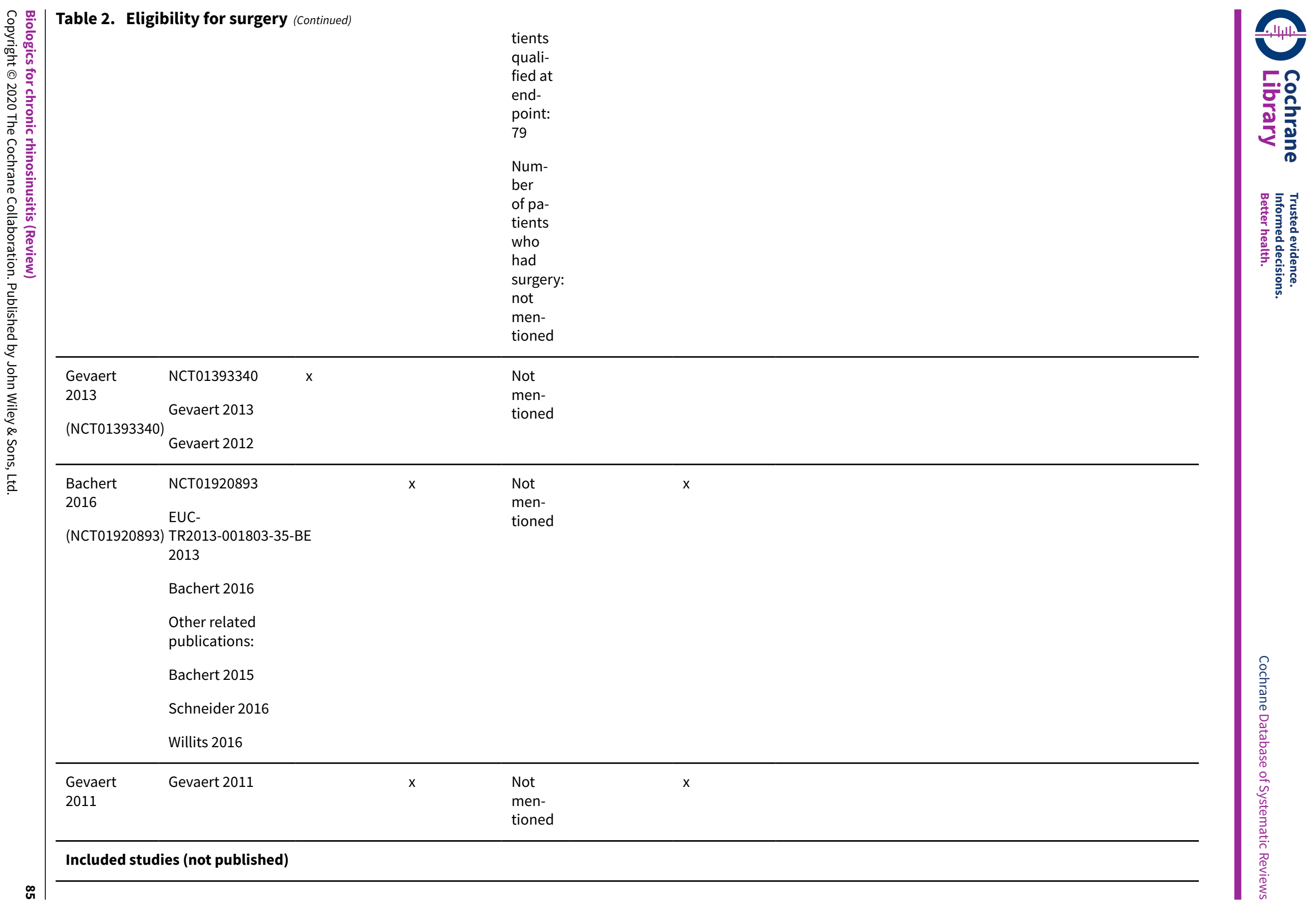




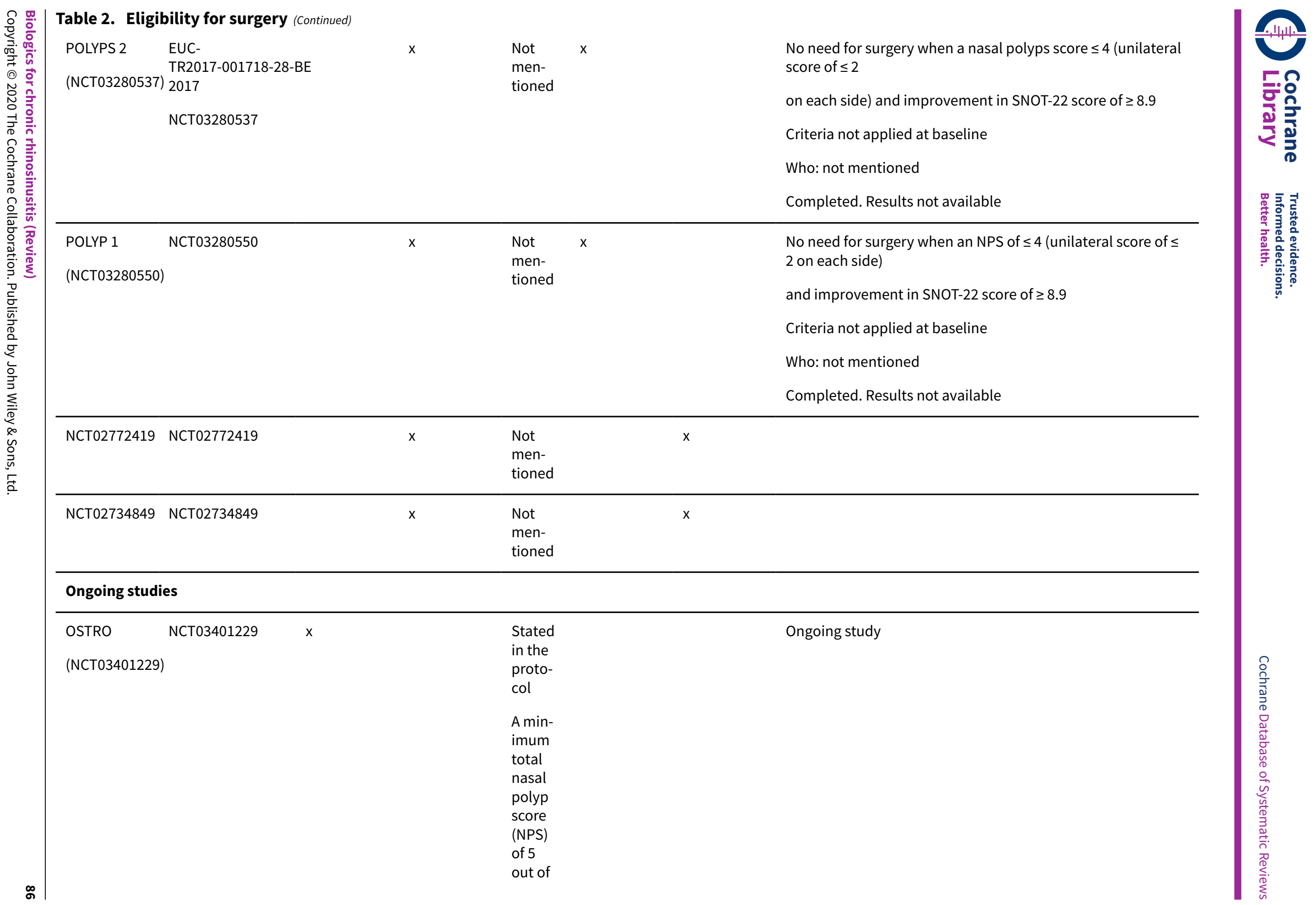




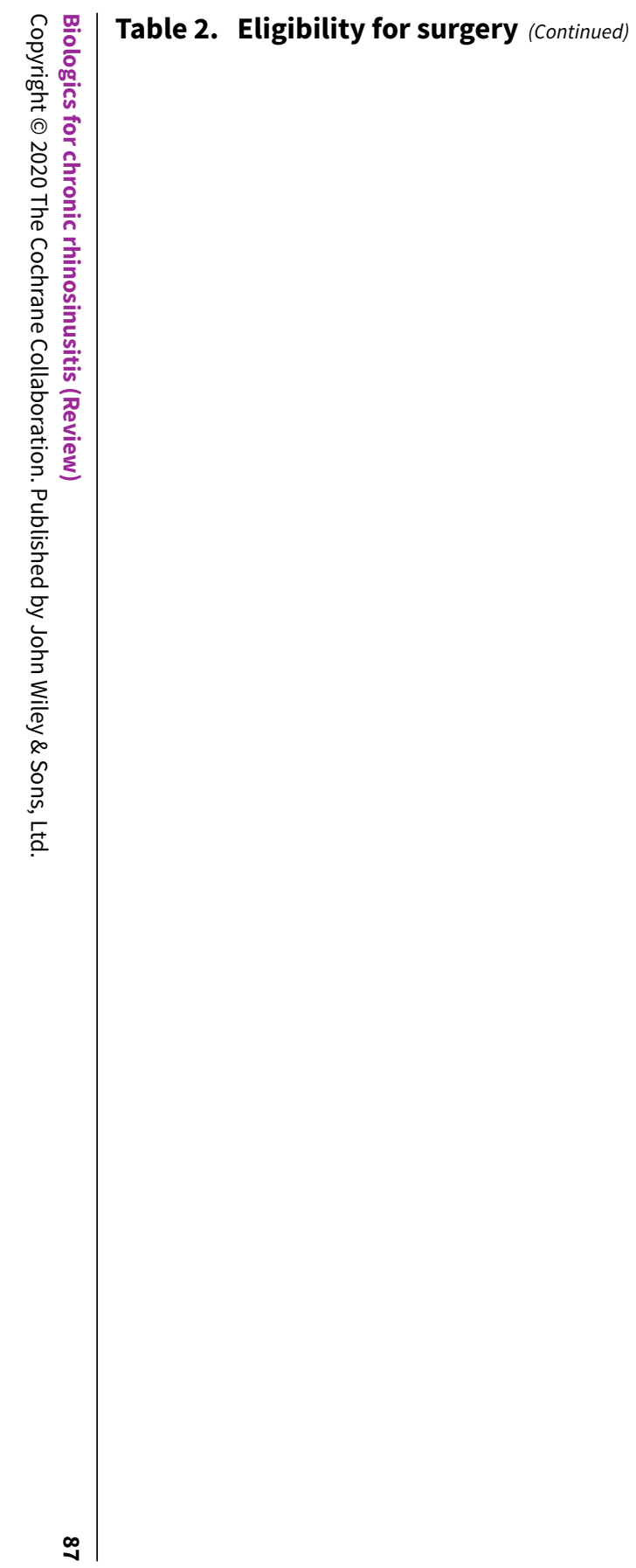

a max-

imum

score

of 8

(with

a uni-

latera

score

least

2 for

each

nos-

tril) at

$V 1$ and

contin-

uously

main-

at V2

to

meet

the

ran-

domi-

sation

criteri-

on, as

deter-

mined

by the

study

Imag-

ing

Core

Lab

On-

going

symp

toms

for at

least

12

weeks

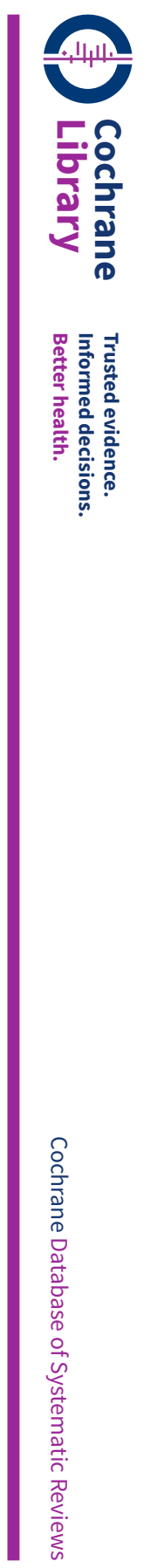




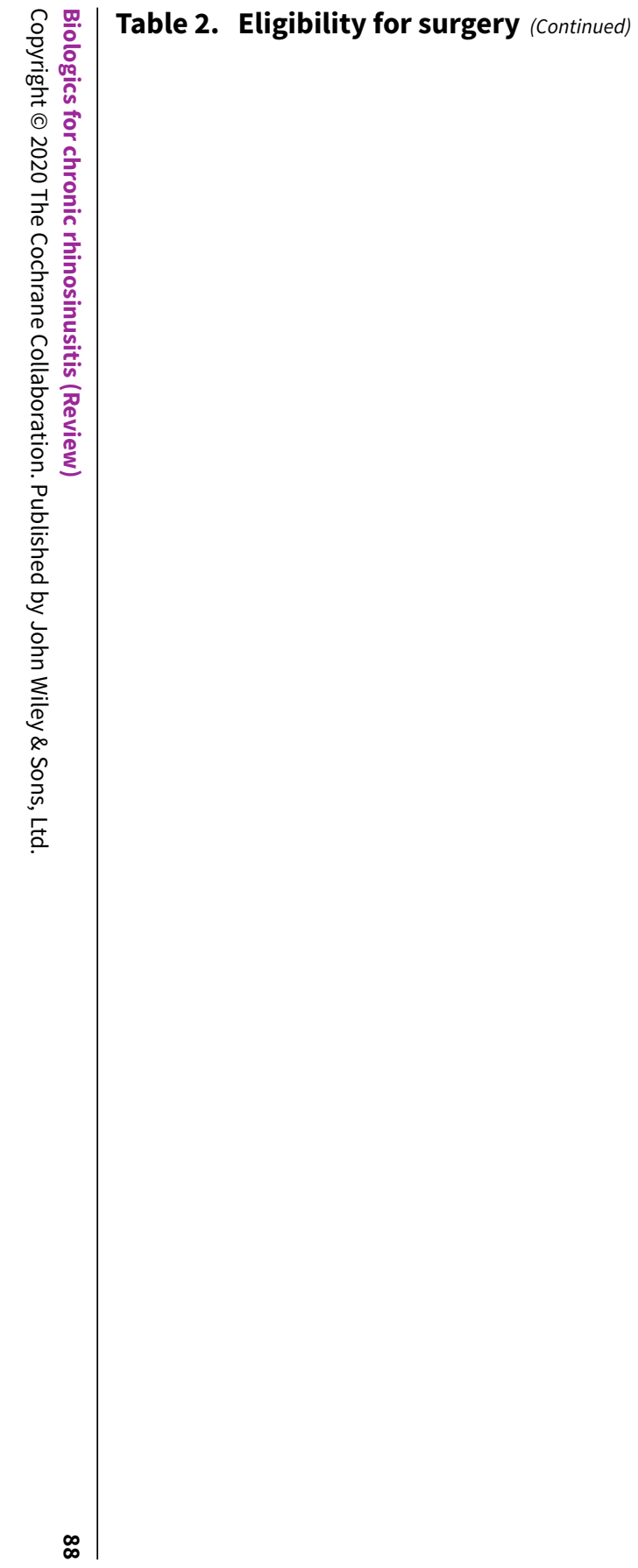

prior to 


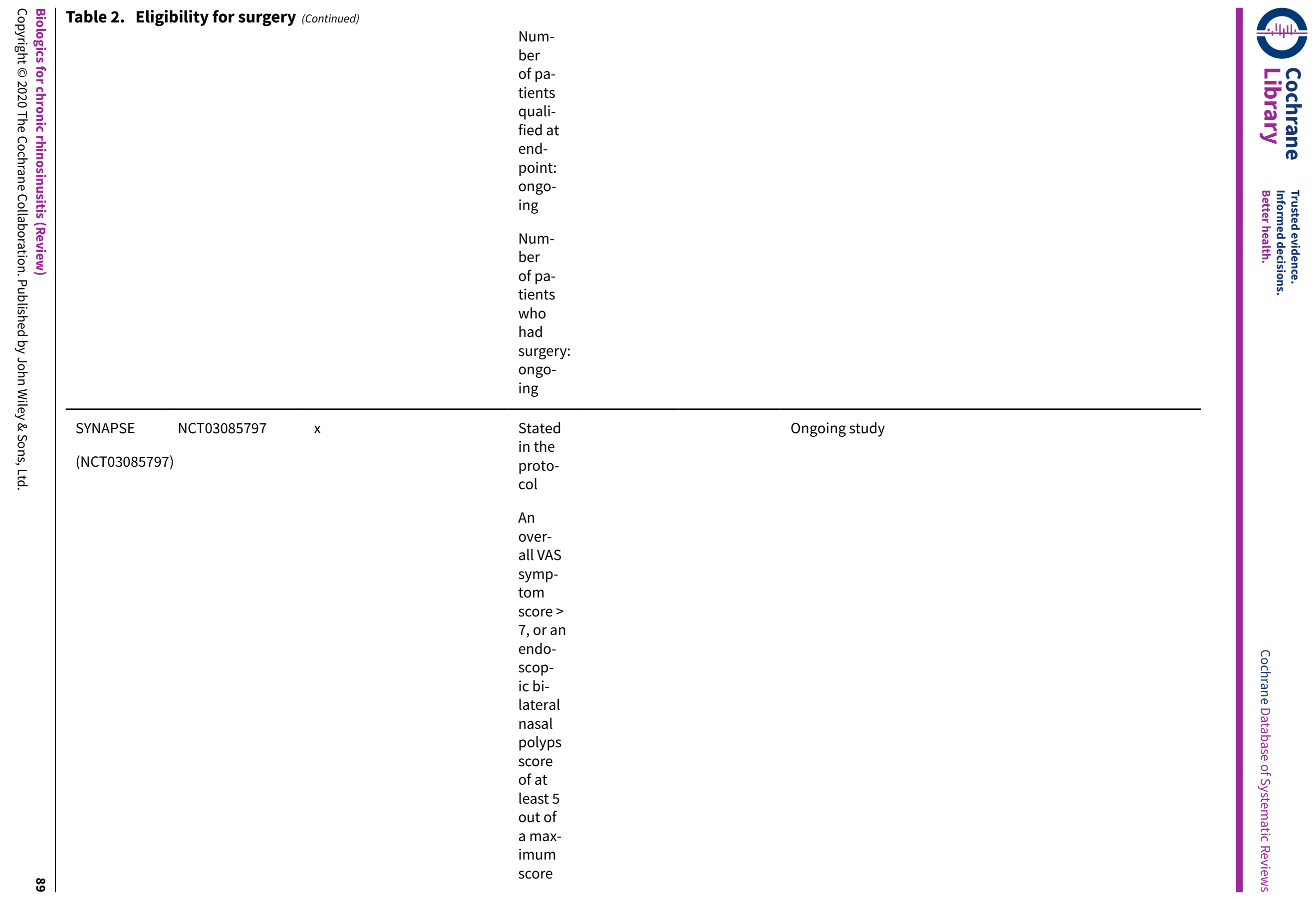



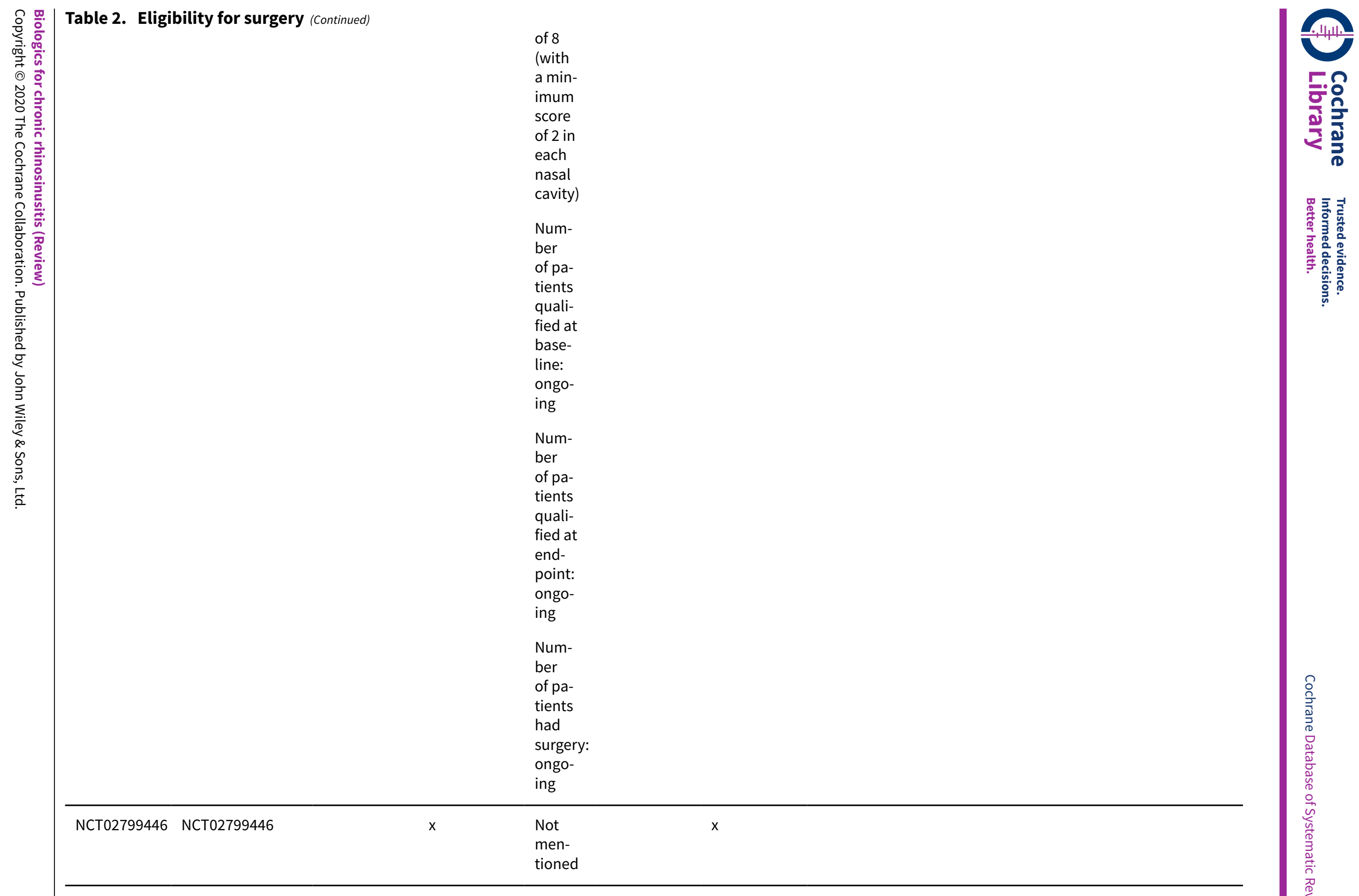


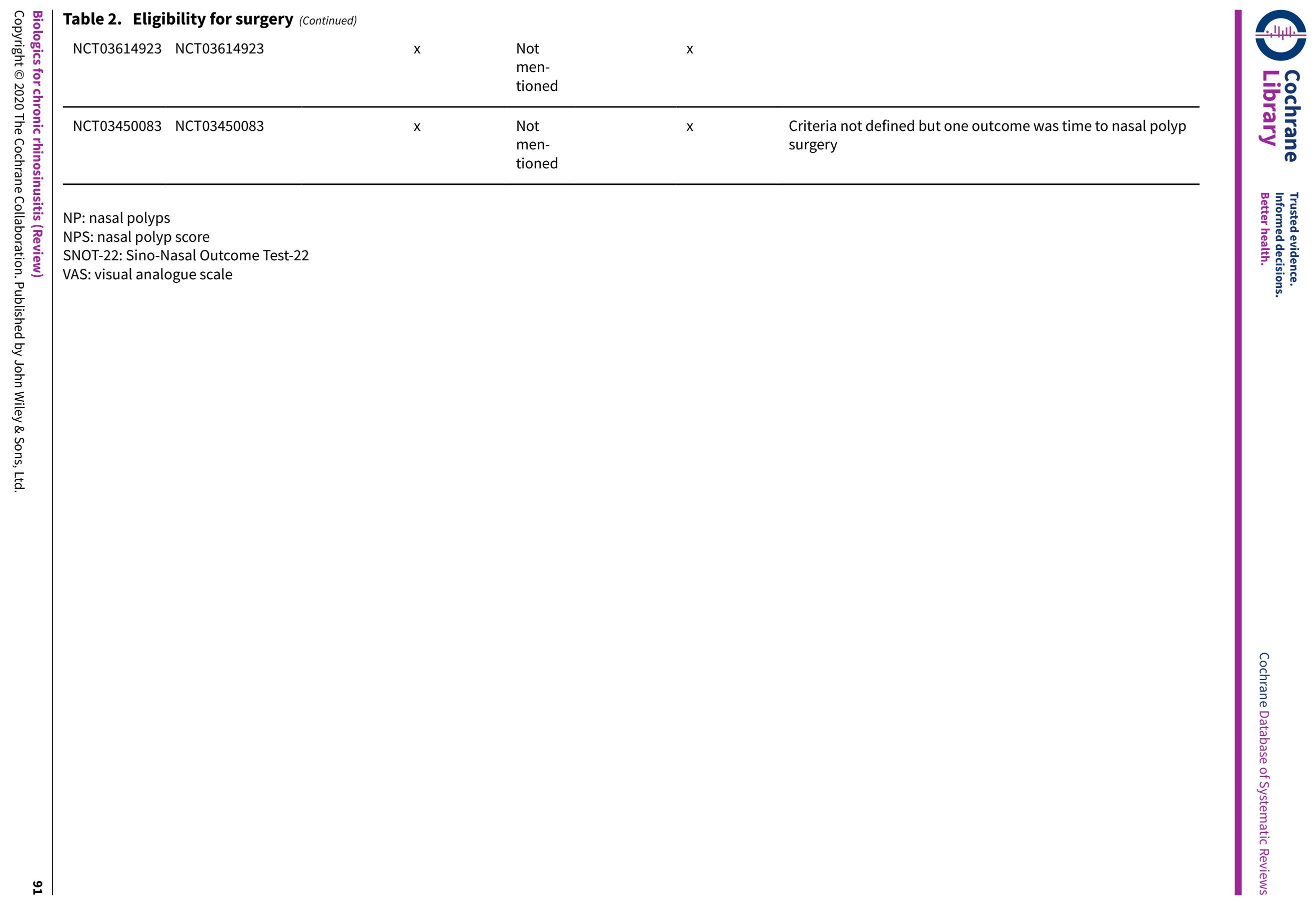




\section{AP PENDICES}

\section{Appendix 1. Search strategies (main electronic sources)}

\begin{tabular}{|c|c|c|c|}
\hline CENTRAL (via CRS) & ENT Register (via CRS) & MEDLINE (Ovid) & Embase (Ovid) \\
\hline $\begin{array}{l}1 \text { MESH DESCRIPTOR Sinusitis EX- } \\
\text { PLODE ALL AND CENTRAL:TARGET }\end{array}$ & $\begin{array}{l}1 \text { MESH DESCRIPTOR Si- } \\
\text { nusitis EXPLODE ALL AND }\end{array}$ & $\begin{array}{l}1 \text { exp Sinusitis/ } \\
2 \text { paranasal sinus dis- }\end{array}$ & $\begin{array}{l}1 \text { exp sinusitis/ or paranasal sinus } \\
\text { disease/ }\end{array}$ \\
\hline 2 MESH DESCRIPTOR Rhinitis AND & & eases/or rhinitis/ or & 2 rhinitis/ or atrophic rhinitis/ or \\
\hline CENTRAL:TARGET & 2 MESH DESCRIPTOR & rhinitis, atrophic/ or & chronic rhinitis/ or rhinosinusitis/ \\
\hline 3 MESH DESCRIPTOR Rhinitis, Atroph- & Rhinitis AND INREGI & rhinitis, vasomotor/ & vasomotor rhinitis/ \\
\hline ic AND CENTRAL:TARGET & $\begin{array}{l}3 \text { MESH DESCRIPTOR } \\
\text { Rhinitis, Atrophic AND IN- }\end{array}$ & 3 exp Paranasal Sinuses/ & 3 exp paranasal sinus/ \\
\hline $\begin{array}{l}4 \text { MESH DESCRIPTOR Rhinitis, Vaso- } \\
\text { motor AND CENTRAL:TARGET }\end{array}$ & REGISTER & $\begin{array}{l}4 \text { (rhinosinusitis or na- } \\
\text { sosinusitis or pansi- }\end{array}$ & $\begin{array}{l}4 \text { (rhinosinusitis or nasosinusitis } \\
\text { or pansinusitis or ethmoiditis or }\end{array}$ \\
\hline 5 MESH DESCRIPTOR Paranasal Sinus & $\begin{array}{l}4 \text { MESH DESCRIPTOR } \\
\text { Rhinitis, Vasomotor AND }\end{array}$ & $\begin{array}{l}\text { nusitis or ethmoiditis or } \\
\text { sphenoiditis).ab,ti. }\end{array}$ & sphenoiditis).tw. \\
\hline CENTRAL:TARGET & INREGISTER & ग.वv, प. & $\begin{array}{l}5 \text { (kartagener }^{*} \text { adj3 syn- } \\
\text { drome*).tw. }\end{array}$ \\
\hline 6 MESH DESCRIPTOR Paranasal & $5 \mathrm{MESH}$ DES & drome*).ab,ti. & \\
\hline Sinuses EXPLODE ALL AND CEN- & Paranasal Sinus Diseases & & 6 (inflamm adj5 sinus*).tw. \\
\hline TRAL:TARGET & AND INREGISTER & $\begin{array}{l}6 \text { (inflamm* adj5 si- } \\
\text { nus*).ab,ti. }\end{array}$ & 7 ((maxilla* or frontal*) adj3 si- \\
\hline 7 (rhinosinusitis or nasosinusitis or & 6 MESH DESCRIPTOR & & nus $\left.{ }^{\star}\right) . t w$. \\
\hline pansinusitis or ethmoiditis or sphe- & $\begin{array}{l}\text { Paranasal Sinuses EX- } \\
\text { PLODE ALL AND IN- }\end{array}$ & $\begin{array}{l}7\left(\left(\text { maxilla }^{\star} \text { or frontal }\right.\right. \\
\left.\text { adj3 sinus }{ }^{\star}\right) \cdot a b, t i .\end{array}$ & 81 or 2 or 3 or 4 or 5 or 6 or 7 \\
\hline AND CENTRAL:TARGET & REGISTER & 81 or 2 or 3 & 9 exp chronic disease/ \\
\hline $\begin{array}{l}\left.8 \text { (kartagener }{ }^{\star} \text { near syndrome }{ }^{\star}\right): A B, E- \\
\text { H,KW,KY,MC,MH,TI,TO AND CEN- }\end{array}$ & $\begin{array}{l}7 \text { (rhinosinusitis or na- } \\
\text { sosinusitis or pansi- }\end{array}$ & or 7 & 10 exp recurrent disease/ \\
\hline TRAL:TARGET & nusitis or ethmoiditis or & 9 exp chronic disease/ & 11 (chronic or persis ${ }^{\star}$ or recur ${ }^{\star}$ ).tw. \\
\hline 9 (inflamm* near sinus*):AB,E- & $\begin{array}{l}\text { sphenoiditis):AB,EH,K- } \\
\text { W,KY,MC,MH,TI,TO AND }\end{array}$ & 10 exp Recurrence/ & 129 or 10 or 11 \\
\hline 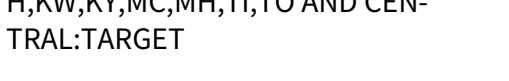 & INREGISTER & $\begin{array}{l}11 \text { (chronic or persis }{ }^{\star} \text { or } \\
\left.\text { recur }^{\star}\right) \cdot \text { ab,ti. }\end{array}$ & 138 and 12 \\
\hline $\begin{array}{l}10\left(\left(\text { maxilla* }^{*} \text { or frontal }{ }^{\star}\right) \text { near si- }\right. \\
\left.\text { nus }{ }^{\star}\right): A B, E H, K W, K Y, M C, M H, T I, T O ~ A N D\end{array}$ & $\begin{array}{l}8 \text { (kartagener }^{\star} \text { near syn- } \\
\text { drome*):AB,EH,KW,KY,M- }^{*} \text { C,MH,TI,TO AND IN- }\end{array}$ & 129 or 10 or 11 & 14 CRSsNP.tw. \\
\hline CENTRAL:TARGET & REGISTER & 138 and 12 & $\begin{array}{l}15 \text { ((sinusitis or rhinitis) adj3 } \\
\left.\left.\text { (chronic or persis* } \text { or recur }^{\star}\right)\right) . \text { tw. }\end{array}$ \\
\hline $\begin{array}{l}11 \# 1 \text { or } \# 2 \text { or } \# 3 \text { or } \# 4 \text { or } \# 5 \text { or } \# 6 \\
\text { or } \# 7 \text { or } \# 8 \text { or } \# 9 \text { or } \# 10 \text { AND CEN- }\end{array}$ & 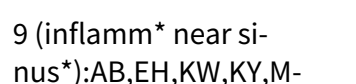 & & 1613 or 14 or 15 \\
\hline $\begin{array}{l}\text { TRAL:TARGET } \\
12 \text { MESH DESCRIPTOR Chronic }\end{array}$ & $\begin{array}{l}\text { C,MH,TI,TO AND IN- } \\
\text { REGISTER }\end{array}$ & 15 ((sinusitis or rhinitis) & 17 exp nose polyp/ \\
\hline $\begin{array}{l}\text { Disease EXPLODE ALL AND CEN- } \\
\text { TRAL:TARGET }\end{array}$ & $\begin{array}{l}10\left(\left(\text { maxilla* }^{*} \text { or frontal }\right.\right. \\
\text { near sinus })\end{array}$ & 1613 or 14 or 15 & $\begin{array}{l}18 \text { exp nose disease/ or exp nose/ } \\
19 \text { exp polyp/ }\end{array}$ \\
\hline $\begin{array}{l}13 \text { MESH DESCRIPTOR Recurrence } \\
\text { EXPLODE ALL AND CENTRAL:TARGET }\end{array}$ & $\begin{array}{l}\text { W,KY,MC,MH,TI,TO AND } \\
\text { INREGISTER }\end{array}$ & 17 exp Nasal Polyps/ & 2018 and 19 \\
\hline $\begin{array}{l}\left.14 \text { (chronic or persis* or recur }{ }^{\star}\right): A B, E- \\
\text { H,KW,KY,MC,MH,TI,TO AND CEN- } \\
\text { TRAL:TARGET }\end{array}$ & $\begin{array}{l}11 \# 1 \text { or } \# 2 \text { or } \# 3 \text { or } \# 4 \text { or } \\
\# 5 \text { or } \# 6 \text { or } \# 7 \text { or } \# 8 \text { or } \# 9 \\
\text { or \#10 AND INREGISTER }\end{array}$ & $\begin{array}{l}18 \text { exp Nose/ or exp Nose } \\
\text { Diseases/ } \\
19 \text { exp Polyps/ }\end{array}$ & $\begin{array}{l}21 \text { ((nose or nasal or rhino* or } \\
\text { rhinitis or sinus* or sinonasal) adj3 } \\
\left.\left(\text { papilloma* or polyp }{ }^{\star}\right)\right) . \text { tw. }\end{array}$ \\
\hline $15 \# 12$ or \#13 or \#14 AND CEN- & $\begin{array}{l}12 \text { MESH DESCRIPTOR } \\
\text { Chronic Disease EX- }\end{array}$ & 2018 and 19 & 22 (rhinopolyp* or CRSwNP).tw. \\
\hline & PLODE ALL AND IN- & 21 & 2316 or 17 or 20 or 21 or 22 \\
\hline $\begin{array}{l}16 \# 11 \text { and \#15 AND CENTRAL:TAR- } \\
\text { GET }\end{array}$ & REGISTER & no* or rhinitis or sinus* & 24 exp antiidiotypic antibody/ \\
\hline
\end{tabular}


(Continued)

17 (CRSsNP):AB,EH,KW,KY,MC,MH,TI,TO AND CENTRAL:TARGET

18 ((sinusitis or rhinitis) near (chronic or persis* or recur $\left.{ }^{\star}\right)$ ):AB,EH, KW, KY,MC,MH,TI,TO AND CENTRAL:TARGET

$19 \# 16$ or \#17 or \#18 AND CENTRAL:TARGET

20 MESH DESCRIPTOR Nasal Polyps EXPLODE ALL AND CENTRAL:TARGET

21 MESH DESCRIPTOR Nose EXPLODE ALL AND CENTRAL:TARGET

22 MESH DESCRIPTOR Nose Diseases EXPLODE ALL AND CENTRAL:TARGET

\section{3 \#21 or \#22 AND CENTRAL:TARGET}

24 MESH DESCRIPTOR Polyps EXPLODE ALL AND CENTRAL:TARGET

25 \#23 and \#24 AND CENTRAL:TARGET

26 ((nose or nasal or rhino* or rhinitis or sinus* or sinonasal) near (papilloma* or polyp*)):AB,EH,KW,KY,MC,MH,TI,TO AND CENTRAL:TARGET

27 (rhinopolyp* or CRSwNP):AB,EH,KW,KY,MC,MH,TI,TO AND CENTRAL:TARGET

$28 \# 19$ or \#20 or \#25 or \#26 or \#27 AND CENTRAL:TARGET

29 MESH DESCRIPTOR Antibodies, Monoclonal EXPLODE ALL AND CENTRAL:TARGET

30 MESH DESCRIPTOR Antibodies, Anti-Idiotypic EXPLODE ALL AND CENTRAL:TARGET

31 MESH DESCRIPTOR Immunoglobulin E EXPLODE ALL AND CENTRAL:TARGET

32 MESH DESCRIPTOR Interleukins EXPLODE ALL AND CENTRAL:TARGET

33 MESH DESCRIPTOR Receptors, Interleukin EXPLODE ALL AND CENTRAL:TARGET

34 MESH DESCRIPTOR Biological Therapy EXPLODE ALL AND CENTRAL:TARGET
13 MESH DESCRIPTOR Recurrence EXPLODE ALL AND INREGISTER

14 (chronic or persis ${ }^{*}$ or recur $\left.^{\star}\right): A B, E H, K W, K Y, M-$ C,MH,TI,TO AND INREGISTER

15 \#12 or \#13 or \#14 AND INREGISTER

$16 \# 11$ and \#15 AND INREGISTER

17 (CRSsNP):AB,EH,KW,KY,MC,MH,TI,TO AND INREGISTER

18 ((sinusitis or rhinitis) near (chronic or persis $^{\star}$ or recur $\left.\left.{ }^{\star}\right)\right): A B, E H, K-$ W,KY,MC,MH,TI,TO AND INREGISTER

$19 \# 16$ or \#17 or \#18 AND INREGISTER

20 MESH DESCRIPTOR Nasal Polyps EXPLODE ALL AND INREGISTER

21 MESH DESCRIPTOR Nose EXPLODE ALL AND INREGISTER

22 MESH DESCRIPTOR Nose Diseases EXPLODE ALL AND INREGISTER

23 \#21 or \#22 AND INREGISTER

24 MESH DESCRIPTOR Polyps EXPLODE ALL AND INREGISTER

25 \#23 and \#24 AND INREGISTER

26 ((nose or nasal or rhino* or rhinitis or sinus* or sinonasal) near (papilloma* or polyp $\left.{ }^{\star}\right)$ ):AB,E$\mathrm{H}, \mathrm{KW}, \mathrm{KY}, \mathrm{MC}, \mathrm{MH}, \mathrm{TI}, \mathrm{TO}$ AND INREGISTER

27 (rhinopolyp* or CRSWNP):AB,EH,KW, KY,MC,MH,TI,TO AND INREGISTER or sinonasal) adj3 (papilloma* or polyp*)).ab,ti.

22 (rhinopolyp* or

CRSwNP).ab,ti.

2316 or 17 or 20 or 21 or 22

24 exp Antibodies, Mono clonal/

25 exp Antibodies, Anti-Idiotypic/

26 exp Immunoglobulin E/

\section{7 exp INTERLEUKINS/}

28 exp Receptors, Interleukin/

29 exp Biological Therapy/

30 exp Granulocyte-Macrophage Colony-Stimulating Factor/

31 exp Cytokines/

32 exp Etanercept/ or exp Alefacept/

33 (Antibod* adj3 monoclonal).ab,ti.

34 (Interleukin* or IgE or "immunoglobulin E" or Antiglobulin* or antiidiotyp*).ab,ti.

35 (anti adj3 (globulin* or idiotyp* or immunoglobulin* or M1 or CCR4 or "LFA 1" or "GATA 3" or OX40L)).ab,ti.

36 (ralokimumab or Adalimumab or Alemtuzumab or Bevacizumab or Certolizumab or Cetuximab or Denosumab or Ipilimumab or Natalizumab or Omalizumab or Palivizumab or Ranibizumab or Trastuzumab or stekinumab or mepolizumab or Nucala or SB240563 or "SB 240563" or dupilumab or REGN668
25 biological product/

26 exp immunoglobulin e/

27 exp interleukin derivative/

28 exp interleukin receptor/

29 exp monoclonal antibody/

30 exp chemokine receptor CCR4 antagonist/

31 exp cytokine/

32 biological factor/

33 exp cytokine receptor antagonist/

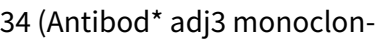
al).ab,ti.

35 (Interleukin* or IgE or "immunoglobulin E" or Antiglobulin* or antiidiotyp*).ab,ti.

36 (anti adj3 (globulin* or idiotyp* or immunoglobulin* or M1 or CCR4 or "LFA 1 " or "GATA 3 " or OX40L)).ab,ti.

37 (ralokimumab or Adalimumab or Alemtuzumab or Bevacizumab or Certolizumab or Cetuximab or Denosumab or Ipilimumab or Natalizumab or Omalizumab or Palivizumab or Ranibizumab or Trastuzumab or stekinumab or mepolizumab or Nucala or SB240563 or "SB 240563" or dupilumab or REGN668 or AMG317 or "AMG 317 " or AMG827 or "AMG 827 " or DNAzyme or antiTSLP or CSL311 or "CSL 311" or "AMG 761" or AMG761 or "AMG 837" or KW0761 or "KW 0761" or "CSF 2" or "CSF GM").ab.

38 (siliq or D2E7 or humira or campath or Lemtrada or avastin or cimzia or CDP870 or "CDP 870" or Erbitux or C225 or Xgeva or prolia or "AMG 162" or AMG162 or Yervoy or Tysabri or Antegren or Xolair* or Synagis or RhuFab or lucentis or Herceptin or stelara or CNTO or ASM8 or granulocyte-macrophage or GM-CSF or QGE031 or Raptiva or AK001 or "AK 001").ab,ti. 
(Continued)

35 MESH DESCRIPTOR Granulocyte-Macrophage Colony-Stimulating Factor EXPLODE ALL AND CENTRAL:TARGET

36 MESH DESCRIPTOR Cytokines EXPLODE ALL AND CENTRAL:TARGET

37 MESH DESCRIPTOR Etanercept EXPLODE ALL AND CENTRAL:TARGET

38 MESH DESCRIPTOR Immunoglobulin G EXPLODE ALL AND CENTRAL:TARGET

39 (Antibod* ${ }^{*}$ adj3 monoclonal):AB,E$\mathrm{H}, \mathrm{KW}, \mathrm{KY}, \mathrm{MC}, \mathrm{MH}, \mathrm{TI}, \mathrm{TO}$ AND CENTRAL:TARGET

40 (Interleukin* or IgE or "immunoglobulin E" or Antiglobulin* or antiidiotyp $\left.{ }^{\star}\right): A B, E H, K W, K Y, M-$ C,MH,TI,TO AND CENTRAL:TARGET

41 (anti adj3 (globulin* or idiotyp* or immunoglobulin * or M1 or CCR4 or "LFA 1" or "GATA 3" or OX40L)):AB,E$\mathrm{H}, \mathrm{KW}, \mathrm{KY}, \mathrm{MC}, \mathrm{MH}, \mathrm{TI}, \mathrm{TO}$ AND CENTRAL:TARGET

42 (ralokimumab or Adalimumab or Alemtuzumab or Bevacizumab or Certolizumab or Cetuximab or Denosumab or Ipilimumab or Natalizumab or Omalizumab or Palivizumab or Ranibizumab or Trastuzumab or stekinumab or mepolizumab or Nucala or SB240563 or "SB 240563" or dupilumab or REGN668 or AMG317 or "AMG 317 " or AMG827 or "AMG 827" or DNAzyme or antiTSLP or CSL311 or "CSL 311" or "AMG 761" or AMG761 or "AMG 837" or KW0761 or "KW 0761" or "CSF 2" or "CSF GM"):AB,E$\mathrm{H}, \mathrm{KW}, \mathrm{KY}, \mathrm{MC}, \mathrm{MH}, \mathrm{TI}, \mathrm{TO}$ AND CENTRAL:TARGET

43 (siliq or D2E7 or humira or campath or Lemtrada or avastin or cimzia or CDP870 or "CDP 870" or Erbitux or C225 or Xgeva or prolia or "AMG 162" or AMG162 or Yervoy or Tysabri or Antegren or Xolair or Synagis or RhuFab or lucentis or Herceptin or stelara or CNTO or ASM8 or granulocyte-macrophage or GM-CSF or QGE031 or Raptiva or AK001 or "AK 001"):AB,EH,KW,KY,MC,MH,TI,TO AND CENTRAL:TARGET

44 ((B-cell or T-cell or Eosinophil or "mast cell" or stimulating) adj3 fac-
28 \#19 or \#20 or \#25 or \#26 or \#27 AND INREGISTER

29 MESH DESCRIPTOR Antibodies, Monoclonal EXPLODE ALL AND INREGISTER

30 MESH DESCRIPTOR Antibodies, Anti-Idiotypic EXPLODE ALL AND INREGISTER

\section{MESH DESCRIPTOR} Immunoglobulin E EXPLODE ALL AND INREGISTER

32 MESH DESCRIPTOR Interleukins EXPLODE ALL AND INREGISTER

33 MESH DESCRIPTOR Receptors, Interleukin EXPLODE ALL AND INREGISTER

\section{MESH DESCRIPTOR} Biological Therapy EXPLODE ALL AND INREGISTER

\section{MESH DESCRIPTOR} Granulocyte-Macrophage Colony-Stimulating Factor EXPLODE ALL AND INREGISTER

36 MESH DESCRIPTOR Cytokines EXPLODE ALL AND INREGISTER

37 MESH DESCRIPTOR Etanercept EXPLODE ALL AND INREGISTER

38 MESH DESCRIPTOR Immunoglobulin G EXPLODE ALL AND INREGISTER

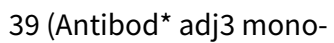
clonal):AB, EH, KW, KY,MC,MH,TI,TO AND INREGISTER

40 (Interleukin* or IgE or "immunoglobulin E" or Antiglobulin* or antiidiotyp $\left.{ }^{\star}\right): A B, E H, K-$ W, KY,MC,MH,TI,TO AND INREGISTER or AMG827 or "AMG 827" or DNAzyme or antiTSLP or CSL311 or "CSL 311" or "AMG 761" or AMG761 or "AMG 837" or KW0761 or "KW 0761" or "CSF 2" or "CSF GM").ab,ti.

37 (siliq or D2E7 or humira or campath or Lemtrada or avastin or cimzia or CDP870 or "CDP 870" or Erbitux or C225 or Xgeva or prolia or "AMG 162" or AMG162 or Yervoy or Tysabri or Antegren or Xolair or Synagis or RhuFab or lucentis or Herceptin or stelara or CNTO or ASM 8 or granulocyte-macrophage or GM-CSF or QGE031 or Raptiva or AK001 or "AK 001").ab,ti.

38 ((B-cell or T-cell or Eosinophil or "mast cell" or stimulating) adj3 factor).ab,ti.

39 (CD23 or CD2 or CD11a or CD20 or CD25 opr CD252 or (receptor ${ }^{\star}$ adj3 apsilon)).ab,ti.

40 (CD adj3 ("23" or antigen* or "2" or 11a or "20" or "25" or "252")).ab,ti.

41 ((antigamma or "anti gamma") adj3 Anti-

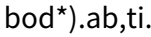

42 (IgEid or "55700" or SCH55700 or CEP38072 or "CEP 38072" or cinqair or DCP835 or "DCP 835" or GM-CSF or TNF or TSLP or OX40L).ab,ti.

43 (IL adj3 ("5" or five or "4" or four or "13" or thirteen or "1" or one or "10" or ten or "11" or eleven or "12" or twelve or "15" or fifteen or "16" or sixteen or "17" or seventeen or "18" or eighteen or "2" or two or "23" or "twenty three" or "12" or twelve
39 ((B-cell or T-cell or Eosinophil or "mast cell" or stimulating) adj3 factor).ab,ti.

40 (CD23 or CD2 or CD11a or CD20 or CD25 opr CD252 or (receptor* adj3 apsilon)).ab,ti.

41 (CD adj3 ("23" or antigen* or "2" or 11a or "20" or "25" or "252")).ab,ti.

42 ((antigamma or "anti gamma") adj3 Antibod*).ab,ti.

43 (IgEid or "55700" or SCH55700 or CEP38072 or "CEP 38072" or cinqair or DCP835 or "DCP 835" or GM-CSF or TNF or TSLP or OX40L).ab,ti.

44 (IL adj3 ("5" or five or "4" or four or "13" or thirteen or "1" or one or "10" or ten or "11" or eleven or "12" or twelve or "15" or fifteen or "16" or sixteen or "17" or seventeen or "18" or eighteen or "2" or two or "23" or "twenty three" or "12" or twelve or " 27 " or "twenty seven" or "3" or three or "33" or "thirty three" or "6" or six or "7" or seven or "8" or eight or "9" or nine or $5 R^{\star}$ or $1 \mathrm{R} 1$ or $4 R^{\star}$ or $12 \mathrm{p} 40$ or IL-23p40 or 17A or 17RA or "22" or "twenty two" or "31" or "thirty one" or 31R)).ab,ti.

45 (IL5 or IL4 or IL13 or IL1 or IL10 or IL11 or IL12 or IL15 or IL15 or IL16 or IL17 or IL18 or IL2 or IL23 or IL12 or IL27 or IL3 or IL33 or IL6 or IL7 or IL8 or IL9 or IL22 or IL31).ab,ti.

46 (biologic or biologics or biotherap*).ab,ti.

47 (biologic* adj3 therap*).ab,ti.

48 ( $\mathrm{mAB}$ or mepo or MDX or MEDI or siglec* or "lectin 8").ab,ti.

49 (SAR231893 or reslizumab or siglec 8 or benralizumab or lebrikizumab or brodalumab or Tralokinumab or Quilizumab or Ligelizumab or Mogamulizumab or Efalizumab or Pitrakinra or Odulimomab or Mogamulizumabor or BCGF or binetrakin or "anti antibod*").ab,ti. 
(Continued)

tor):AB,EH,KW,KY,MC,MH,TI,TO AND CENTRAL:TARGET

45 (CD23 or CD2 or CD11a or CD20 or CD25 opr CD252 or (receptor* adj3 apsilon)):AB,EH, KW, KY,MC,MH,TI,TO AND CENTRAL:TARGET

46 (CD adj3 ("23" or antigen* or "2" or 11a or "20" or "25" or "252")):AB,EH,KW,KY,MC,MH,TI,TO AND CENTRAL:TARGET

47 ((antigamma or "anti gamma") adj3 Antibod $\left.^{\star}\right): A B, E H, K W, K Y, M-$ C,MH,TI,TO AND CENTRAL:TARGET

48 (IgEid or "55700" or SCH55700 or CEP38072 or "CEP 38072" or cinqair or DCP835 or "DCP 835" or GMCSF or TNF or TSLP or OX40L):AB,E$\mathrm{H}, \mathrm{KW}, \mathrm{KY}, \mathrm{MC}, \mathrm{MH}, \mathrm{TI}, \mathrm{TO}$ AND CENTRAL:TARGET

49 (IL adj3 ("5" or five or "4" or four or "13" or thirteen or "1" or one or "10" or ten or "11" or eleven or "12" or twelve or "15" or fifteen or "16" or sixteen or "17" or seventeen or "18" or eighteen or "2" or two or "23" or "twenty three" or "12" or twelve or "27" or "twenty seven" or "3" or three or "33" or "thirty three" or "6" or six or "7" or seven or "8" or eight or "9" or nine or $5 \mathrm{R}^{\star}$ or $1 \mathrm{R} 1$ or $4 \mathrm{R}^{\star}$ or $12 \mathrm{p} 40$ or IL-23p40 or 17A or 17RA or "22" or "twenty two" or "31" or "thirty one" or 31R)):AB,EH,KW,KY,MC,MH,TI,TO AND CENTRAL:TARGET

50 (biologic or biologics or biothera$\left.\mathrm{p}^{\star}\right): \mathrm{AB}, \mathrm{EH}, \mathrm{KW}, \mathrm{KY}, \mathrm{MC}, \mathrm{MH}, \mathrm{TI}, \mathrm{TO}$ AND CENTRAL:TARGET

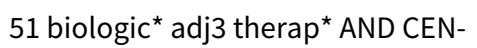
TRAL:TARGET

52 ( $\mathrm{mAB}$ or mepo or MDX or MEDI or siglec* or "lectin 8"):AB,EH,KW,KY,MC,MH,TI,TO AND CENTRAL:TARGET

53 SAR231893 or reslizumab or siglec 8 or benralizumab or lebrikizumab or brodalumab or Tralokinumab or Quilizumab or Ligelizumab or Mogamulizumab or Efalizumab or Pitrakinra or Odulimomab or Mogamulizumabor or BCGF or binetrakin or "anti antibod*" AND CENTRAL:TARGET

54 (Canakinumab or Ilaris or Rilonacept or Arcalyst or Anakinra or
41 (anti adj3 (globulin * or idiotyp* or immunoglobulin* or $\mathrm{M} 1$ or CCR4 or "LFA 1" or "GATA 3" or OX40L)):AB,EH,KW,KY,MC,MH,TI,TO AND INREGISTER

42 (ralokimumab or Adalimumab or Alemtuzumab or Bevacizum$a b$ or Certolizumab or Cetuximab or Denosumab or Ipilimumab or Natalizumab or Omalizumab or Palivizumab or Ranibizumab or Trastuzumab or stekinumab or mepolizumab or Nucala or SB240563 or "SB 240563" or dupilumab or REGN668 or AMG317 or "AMG 317" or AMG827 or "AMG 827" or DNAzyme or antiTSLP or CSL311 or "CSL 311" or "AMG 761" or AMG761 or "AMG 837" or KW0761 or "KW 0761" or "CSF 2" or "CSF GM"):AB,EH,KW,KY,MC,MH,TI,TO AND INREGISTER

43 (siliq or D2E7 or humira or campath or Lemtrada or avastin or cimzia or CDP870 or "CDP 870" or Erbitux or C225 or Xgeva or prolia or "AMG 162" or AMG162 or Yervoy or Tysabri or Antegren or Xolair or Synagis or RhuFab or lucentis or Herceptin or stelara or $\mathrm{CN}$ TO or ASM 8 or granulocyte-macrophage or GM-CSF or QGE031 or Raptiva or AK001 or "AK 001"):AB,EH,KW,KY,MC,MH,TI,TO AND INREGISTER

44 ((B-cell or T-cell or Eosinophil or "mast cell" or stimulating) adj3 factor):AB,EH,KW,KY,MC,MH,TI,TO AND INREGISTER

45 (CD23 or CD2 or CD11a or CD20 or CD25 opr CD252 or (receptor* or "27" or "twenty seven" or "3" or three or "33" or "thirty three" or "6" or six or "7" or seven or "8" or eight or "9" or nine or $5 \mathrm{R}^{\star}$ or $1 \mathrm{R} 1$ or $4 \mathrm{R}^{\star}$ or $12 \mathrm{p} 40$ or IL-23p40 or $17 \mathrm{~A}$ or 17RA or "22" or "twenty two" or "31" or "thirty one" or 31R)).ab,ti.

\section{4 (IL5 or IL4 or IL13 or} IL1 or IL10 or IL11 or IL12 or IL15 or IL15 or IL16 or IL17 or IL18 or IL2 or IL23 or IL12 or IL27 or IL3 or IL33 or IL6 or IL7 or IL8 or IL9 or IL22 or IL31).ab,ti.

45 (biologic or biologics or biotherap $\left.{ }^{\star}\right)$.ab,ti.

46 (biologic $^{*}$ adj3 thera$\left.p^{\star}\right)$.ab,ti.

47 (mAB or mepo or MDX or MEDI or siglec ${ }^{*}$ or "lectin 8").ab,ti.

\section{8 (SAR231893 or} reslizumab or siglec 8 or benralizumab or lebrikizumab or brodalumab or Tralokinumab or Quilizumab or Ligelizumab or Mogamulizumab or Efalizumab or Pitrakinra or Odulimomab or Mogamulizumabor or BCGF or binetrakin or "anti antibod*").ab,ti.

49 (siglec 8 or TPI ASM8 or Rilonacept).rn.

50 (Canakinumab or Ilaris or Rilonacept or Arcalyst or Anakinra or Kineret or Antril or Altrakincept or Nuvance or Pascolizumab or SB 240683 or VAK694 or QBX258 or VAK 694 or VAK-694 or dectrekumab QAX-576 or QAX576 or QAX 576 or aerovant or AER-001 or AER001 or "AER 001" or BAY-16-9996 or BAY 16-9996 or Bosatria or Nucala or CDP 835 or CDP835 or CDP-835 or CINQAIR or CTX55700 or CTX 55700 or CTX-55700
50 (Canakinumab or Ilaris or Rilonacept or Arcalyst or Anakinra or Kineret or Antril or Altrakincept or Nuvance or Pascolizumab or SB 240683 or VAK694 or QBX258 or VAK 694 or VAK-694 or dectrekumab QAX-576 or QAX576 or QAX 576 or aerovant or AER-001 or AER001 or "AER 001" or BAY-16-9996 or BAY 16-9996 or Bosatria or Nucala or CDP 835 or CDP835 or CDP-835 or CINQAIR or CTx 55700 or CTx 55700 or CTx- 55700 or DCP 835 or DCP-835 or DCP835 or SCH5570 or SCH 5570 SCH-5570 or TRFK-5 or TRFK 5 or TRFK5 or BIW-8405* or BIW8405* or BIW $8405^{\star}$ or KHK 4563 or KHK-4563 or KHK4563 or Enokizumab or $7 \mathrm{~F} 3 \mathrm{com}-2 \mathrm{H} 2$ or Ustekinumab or Stelara or CNTO-1275 or CNTO1275 or CNTO 1275 or Anrukinzuma* or IMA-638 or PF-05230917 or GSK679586 or GSK-679586 or GDK 679586 or IMA026 or IMA-026 or "IMA 026" or IMA638 or IMA 638 MILR1444A or MILR 1444A or MILR-1444A or PRO-301444 or PRO301444 or PRO 301444 or RG-3637 or RG3637 or RG 3637 or RO-5490255 or RO5490255 or RO 5490255 or TNX-650 or TNX650 or TNX 650 or RPC-4046 or ABT-308 or RPC 4046 or ABT308 or RPC 4046 or ABT 308 or CAT-354 or CAT354 or CAT 354 or Secukinumab or Cosentyx or AIN-457 or KB-03303A or NVP-AIN 457 or AIN457 or KB03303A or NVPAIN457 or AIN 457 or KB 03303A or NVP-AIN-457 or KHK-4827 or KHK4827 or KHK 4827 or fezakinumab * or ILV-094 or PF-5212367 or ILV094 or PF5212367 or "ILV 094" or PF 5212367 or BMS-981164 or BMS981164 or BMS 981164 or Nemolizumab or CIM331 or CIM 331 or CIM-331 or Lenzilumab or KB003 or "KB 003" or KB-003 or ABT-D2E7 or D2E7 or LU 200134 or ABTD2E7 or LU200134 or ABT D2E7 or LU 200134 or Golimumab or Simponi or CNTO-148 or CNTO148 or CNTO 148 or Inflixima or CA2 or CenTNF or Remicade or TA- 650 or TA 650 or TA 650 or Etanercept or Enbrel or p75TNFR-Ig or rhu TNFR-Fc or TNFR-Fcp75 or TNR-001 or TNR001 or "TNR 001" or AMG-157 or MEDI-9929 or AMG157 or AMG 157 MEDI4212 or MEMP1972A or RG7449 or MEMP 
(Continued)

Kineret or Antril or Altrakincept or Nuvance or Pascolizumab or SB 240683 or VAK694 or QBX258 or VAK 694 or VAK-694 or dectrekumab QAX-576 or QAX576 or QAX 576 or aerovant or AER-001 or AER001 or "AER 001" or BAY-16-9996 or BAY 16-9996 or Bosatria or Nucala or CDP 835 or CDP835 or CDP-835 or CINQAIR or CTx 55700 or CTx 55700 or CTX-55700 or DCP 835 or DCP-835 or DCP835 or SCH5570 or SCH 5570 SCH-5570 or TRFK-5 or TRFK 5 or TRFK5 or BIW-8405* or BIW8405* or BIW $8405^{\star}$ or KHK 4563 or KHK-4563 or KHK4563 or Enokizumab or $7 \mathrm{~F} 3 \mathrm{com}-2 \mathrm{H} 2$ or Ustekinumab or Stelara or CNTO-1275 or CNTO1275 or CNTO 1275 or Anrukinzuma* or IMA-638 or PF-05230917 or GSK679586 or GSK-679586 or GDK 679586 or IMA026 or IMA-026 or "IMA 026" or IMA638 or IMA 638 MILR1444A or MILR 1444A or MILR-1444A or PRO-301444 or PRO301444 or PRO 301444 or RG-3637 or RG3637 or RG 3637 or RO-5490255 or RO5490255 or RO 5490255 or TNX-650 or TNX650 or TNX 650 or RPC-4046 or ABT-308 or RPC 4046 or ABT308 or RPC 4046 or ABT 308 or CAT-354 or CAT354 or CAT 354 or Secukinumab or Cosentyx or AIN-457 or KB-03303A or NVP-AIN 457 or AIN457 or KB03303A or NVPAIN457 or AIN 457 or KB 03303 A or NVP-AIN-457 or KHK-4827 or KHK4827 or KHK 4827 or fezakinumab * or ILV-094 or PF-5212367 or ILV094 or PF5212367 or "ILV 094" or PF 5212367 or BMS-981164 or BMS981164 or BMS 981164 or Nemolizumab or CIM331 or CIM 331 or CIM-331 or Lenzilumab or KB003 or "KB 003 " or KB-003 or ABT-D2E7 or D2E7 or LU 200134 or ABTD2E7 or LU200134 or ABT D2E7 or LU 200134 or Golimumab or Simponi or CNTO-148 or CNTO148 or CNTO 148 or Inflixima or CA2 or CenTNF or Remicade or TA- 650 or TA650 or TA 650 or Etanercept or Enbrel or p75TNFR-Ig or rhu TNFR-Fc or TNFR-Fc-p75 or TNR-001 or TNR001 or "TNR 001" or AMG-157 or MEDI-9929 or AMG157 or AMG 157 MEDI4212 or MEMP1972A or RG7449 or MEMP 1972 A or RG 7449 or MEMP-1972A or RG-7449 or Mogamulizumab or KM8761 or Poteligeo or KM-8761 or KM 8761 or Alefacept or Amevive or "ASP 0485" or BG 9273 or BG 9712 or ASP0485 or BG9273 or BG9712 or ASP-0485 or adj3 apsilon)):AB,EH,KW,KY,MC,MH,TI,TO AND INREGISTER

46 (CD adj3 ("23" or antigen* or "2" or 11a or "20" or "25" or "252")):AB,E$\mathrm{H}, \mathrm{KW}, \mathrm{KY}, \mathrm{MC}, \mathrm{MH}, \mathrm{TI}, \mathrm{TO}$ AND INREGISTER

47 ((antigamma or "anti gamma") adj3 Antibod $\left.^{\star}\right): A B, E H, K W, K Y, M-$ C,MH,TI,TO AND INREGISTER

48 (IgEid or "55700" or SCH55700 or CEP38072 or "CEP 38072" or cinqair or DCP835 or "DCP 835" or GM-CSF or TNF or TSLP or OX40L):AB,EH,KW,KY,MC,MH,TI,TO AND INREGISTER

49 (IL adj3 ("5" or five or "4" or four or "13" or thirteen or " 1 " or one or "10" or ten or "11" or eleven or "12" or twelve or "15" or fifteen or "16" or sixteen or "17" or seventeen or "18" or eighteen or "2" or two or "23" or "twenty three" or "12" or twelve or "27" or "twenty seven" or "3" or three or "33" or "thirty three" or "6" or six or "7" or seven or "8" or eight or "9" or nine or $5 \mathrm{R}^{\star}$ or $1 \mathrm{R} 1$ or $4 \mathrm{R}^{\star}$ or $12 \mathrm{p} 40$ or IL-23p40 or $17 \mathrm{~A}$ or 17RA or "22" or "twenty two" or "31" or "thirty one" or 31R)):AB,EH,KW,KY,MC,MH,TI,TO AND INREGISTER

50 (biologic or biologics or biotherap $\left.{ }^{\star}\right): A B, E H, K-$ W,KY,MC,MH,TI,TO AND INREGISTER

51 biologic* adj3 therap* AND INREGISTER

52 (mAB or mepo or MDX or MEDI or siglec* or "lectin 8"):AB,EH,KW,KY,MC,MH,TI,TO AND INREGISTER or DCP 835 or DCP-835 or DCP835 or SCH5570 or SCH 5570 SCH-5570 or TRFK- 5 or TRFK 5 or TRFK5 or BIW-8405* or BIW8405* or BIW 8405* or KHK 4563 or KHK-4563 or KHK4563 or Enokizumab or $7 \mathrm{~F} 3 \mathrm{com}-2 \mathrm{H} 2$ or Ustekinumab or Stelara or CNTO-1275 or CNTO1275 or CNTO 1275 or Anrukinzuma* or IMA-638 or PF-05230917 or GSK679586 or GSK-679586 or GDK 679586 or IMA026 or IMA-026 or "IMA 026" or IMA638 or IMA 638 MILR1444A or MILR 1444A or MILR-1444A or PRO-301444 or PRO301444 or PRO 301444 or RG-3637 or RG3637 or RG 3637 or RO-5490255 or RO5490255 or RO 5490255 or TNX-650 or TNX650 or TNX 650 or RPC-4046 or ABT-308 or RPC 4046 or $\mathrm{ABT} 308$ or RPC 4046 or ABT 308 or CAT-354 or CAT354 or CAT 354 or Secukinumab or Cosentyx or AIN-457 or KB-03303A or NVP-AIN 457 or AIN457 or KB03303A or NVPAIN457 or AIN 457 or KB 03303A or NVP-AIN-457 or KHK-4827 or KHK4827 or KHK 4827 or fezakinumab * or ILV-094 or PF-5212367 or ILV094 or PF5212367 or "ILV 094" or PF 5212367 or BMS-981164 or BMS981164 or BMS 981164 or Nemolizumab or CIM331 or CIM 331 or CIM-331 or Lenzilumab or KB003 or "KB 003" or KB-003 or ABT-D2E7 or D2E7 or LU 200134 or ABTD2E7 or LU200134 or ABT D2E7 or LU 200134 or Golimumab or Simponi or CNTO-148 or CNTO148 or CNTO 148 or Inflixima or CA2 or CenTNF or Remicade or TA-650
KM8761 or Poteligeo or KM-8761 or KM 8761 or Alefacept or Amevive or "ASP 0485" or BG 9273 or BG 9712 or ASP0485 or BG9273 or BG9712 or ASP-0485 or BG-9273 or BG-9712 or Xanelim or Rituximab or Rituxan or Daclizumab or Zenapax or Oxeluma* or huMAb or OX40L or RG 4930 or R04989991 or RG4930 or RG-4930 or RO 4989991 or RO-4989991 or Bertilimumab or Tezepeluma or Isunakinra or "Fusion Protein ${ }^{\star}$ or cytokine*).ab,ti.

51 or/24-50

5223 and 51

53 (random* $^{\star}$ or factorial ${ }^{*}$ or placebo* or assign ${ }^{\star}$ or allocat ${ }^{\star}$ or crossover ${ }^{\star}$ ).tw.

54 (control* adj group $\left.{ }^{\star}\right)$. tw.

55 (trial $^{*}$ and (control* or comparative)).tw.

56 ((blind ${ }^{*}$ or mask $\left.{ }^{\star}\right)$ and (single or double or triple or treble)).tw.

57 (treatment adj arm*).tw.

58 (control $^{\star}$ adj group ${ }^{\star}$ ).tw.

59 (phase adj (III or three)).tw.

60 (versus or vs).tw.

61 rct.tw.

62 crossover procedure/

63 double blind procedure/

64 single blind procedure/

65 randomization/

66 placebo/

67 exp clinical trial/

68 parallel design/

69 Latin square design/

7053 or 54 or 55 or 56 or 57 or 58 or 59 or 60 or 61 or 62 or 63 or 64 or 65 or 66 or 67 or 68 or 69

71 exp ANIMAL/ or exp NONHUMAN/ or exp ANIMAL EXPERIMENT/ or exp ANIMAL MODEL/

72 exp human/ 
(Continued)

uximab or Rituxan or Daclizumab or Zenapax or Oxeluma* or huMAb or OX40L or RG 4930 or RO4989991 or RG4930 or RG-4930 or RO 4989991 or RO-4989991 or Bertilimumab or Tezepeluma or Isunakinra or "Fusion Protein $^{\star}$ " or cytokine*):AB,EH,KW,KY,MC,MH,TI,TO AND CENTRAL:TARGET

55 (IL5 or IL4 or IL13 or IL1 or IL10 or IL11 or IL12 or IL15 or IL15 or IL16 or IL17 or IL18 or IL2 or IL23 or IL12 or IL27 or IL3 or IL33 or IL6 or IL7 or IL8 or IL9 or IL22 or IL31):AB,EH,KW,KY,MC,MH,TI,TO AND CENTRAL:TARGET

56 \#29 OR \#30 OR \#31 OR \#32 OR \#33 OR \#34 OR \#35 OR \#36 OR \#37 OR \#38 OR \#39 OR \#40 OR \#41 OR \#42 OR \#43 OR \#44 OR \#45 OR \#46 OR \#47 OR \#48 OR \#49 OR \#50 OR \#51 OR \#52 OR \#53 OR \#54 OR \#55

57 \#56 AND \#28
53 SAR231893 or reslizumab or siglec 8 or benralizumab or lebrikizumab or brodalumab or Tralokinumab or Quilizumab or Ligelizumab or Mogamulizumab or Efalizumab or Pitrakinra or Odulimomab or Mogamulizumabor or BCGF or binetrakin or "anti antibod*" AND INREGISTER

54 (Canakinumab or Ilaris or Rilonacept or Arcalyst or Anakinra or Kineret or Antril or Altrakincept or Nuvance or Pascolizumab or SB 240683 or VAK694 or QBX258 or VAK 694 or VAK-694 or dectrekumab QAX-576 or QAX576 or QAX 576 or aerovant or AER-001 or AER001 or "AER 001" or BAY-16-9996 or BAY 16-9996 or Bosatria or Nucala or CDP 835 or CDP835 or CDP-835 or CINQAIR or CTX55700 or CTx 55700 or CTx-55700 or DCP 835 or DCP-835 or DCP835 or SCH5570 or SCH 5570 SCH-5570 or TRFK- 5 or TRFK 5 or TRFK5 or BIW-8405* or BIW8405* or BIW 8405* or KHK 4563 or KHK-4563 or KHK4563 or Enokizumab or $7 \mathrm{~F} 3 \mathrm{com}-2 \mathrm{H} 2$ or Ustekinumab or Stelara or CNTO-1275 or CNTO1275 or CNTO 1275 or Anrukinzuma* or IMA-638 or PF-05230917 or GSK679586 or GSK-679586 or GDK 679586 or IMA026 or IMA-026 or "IMA 026" or IMA638 or IMA 638 MILR1444A or MILR 1444A or MILR-1444A or PRO-301444 or PRO301444 or PRO 301444 or RG-3637 or RG3637 or RG 3637 or RO-5490255 or R05490255 or RO 5490255 or TNX-650 or TNX650 or TNX 650 or RPC-4046 or ABT-308 or TA650 or TA 650 or Etanercept or Enbrel or p75TNFR-Ig or rhu TNFR-Fc or TNFR-Fc-p75 or TNR-001 or TNR001 or "TNR 001" or AMG-157 or MEDI-9929 or AMG157 or AMG 157 MEDI4212 or MEMP1972A or RG7449 or MEMP 1972A or RG 7449 or MEMP-1972A or RG-7449 or Mogamulizumab or KM8761 or Poteligeo or KM-8761 or KM 8761 or Alefacept or Amevive or "ASP 0485" or BG 9273 or BG 9712 or ASP0485 or BG9273 or BG9712 or ASP-0485 or BG-9273 or BG-9712 or Xanelim or Rituximab or Rituxan or Daclizumab or Zenapax or Oxeluma* or huMAb or OX40L or RG 4930 or RO4989991 or RG4930 or RG-4930 or RO 4989991 or RO-4989991 or Bertilimumab or Tezepeluma or Isunakinra or "Fusion Protein ${ }^{\star "}$ or cytokine ${ }^{\star}$ ).ab,ti.

\section{1 or/24-50}

\section{3 and 51}

53 randomized controlled trial.pt.

54 controlled clinical trial.pt.

55 randomized.ab.

56 placebo.ab.

57 drug therapy.fs.

58 randomly.ab.

59 trial.ab.

60 groups.ab.

6153 or 54 or 55 or 56 or 57 or 58 or 59 or 60

62 exp animals/ not humans.sh.

6361 not 62

6452 and 63
7371 not 72

7470 not 73

7552 and 74 
or RPC 4046 or ABT308 or RPC 4046 or ABT 308 or CAT-354 or CAT354 or CAT 354 or Secukinumab or Cosentyx or AIN-457 or KB-03303A or NVP-AIN 457 or AIN457 or KB03303A or NVPAIN457 or AIN 457 or KB 03303A or NVP-AIN-457 or KHK-4827 or KHK4827 or KHK 4827 or fezakinumab * or ILV-094 or PF-5212367 or ILV094 or PF5212367 or "ILV 094" or PF 5212367 or BMS-981164 or BMS981164 or BMS 981164 or Nemolizumab or CIM331 or CIM 331 or CIM-331 or Lenzilumab or KB003 or "KB 003" or KB-003 or ABT-D2E7 or D2E7 or LU 200134 or ABTD2E7 or LU200134 or ABT D2E7 or LU 200134 or Golimumab or Simponi or CNTO- 148 or CNTO148 or CNTO 148 or Inflixima or CA2 or CenTNF or Remicade or TA-650 or TA650 or TA 650 or Etanercept or Enbrel or p75TNFR-Ig or rhu TNFR-Fc or TNFR-Fc-p75 or TNR-001 or TNR001 or "TNR 001" or AMG-157 or MEDI-9929 or AMG157 or AMG 157 MEDI4212 or MEMP1972A or RG7449 or MEMP 1972A or RG 7449 or MEMP-1972A or RG-7449 or Mogamulizumab or KM8761 or Poteligeo or KM-8761 or KM 8761 or Alefacept or Amevive or "ASP 0485" or BG 9273 or BG 9712 or ASP0485 or BG9273 or BG9712 or ASP-0485 or BG-9273 or BG-9712 or Xanelim or Rituximab or Rituxan or Daclizumab or Zenapax or Oxeluma* or huMAb or OX40L or RG 4930 or RO4989991 or RG4930 or RG-4930 or RO 4989991 or RO-4989991 or Bertilimumab or Tezepeluma or Isunakinra or "Fusion Protein*" 
or cytokine $\left.{ }^{\star}\right): A B, E H, K-$ W,KY,MC,MH,TI,TO AND INREGISTER

55 (IL5 or IL4 or IL13 or IL1 or IL10 or IL11 or IL12 or IL15 or IL15 or IL16 or IL17 or IL18 or IL2 or IL23 or IL12 or IL27 or IL3 or IL33 or IL6 or IL7 or IL8 or IL9 or IL22 or IL31):AB,E$\mathrm{H}, \mathrm{KW}, \mathrm{KY}, \mathrm{MC}, \mathrm{MH}, \mathrm{TI}, \mathrm{TO}$ AND INREGISTER

56 \#29 OR \#30 OR \#31 OR \#32 OR \#33 OR \#34 OR \#35 OR \#36 OR \#37 OR \#38 OR \#39 OR \#40 OR \#41 OR \#42 OR \#43 OR \#44 OR \#45 OR \#46 OR \#47 OR \#48 OR \#49 OR \#50 OR \#51 OR \#52 OR \#53 OR \#54 OR \#55 AND INREGISTER

57 \#56 AND \#28 AND INREGISTER

\section{Web of Science (Web of Knowledge)}

\section{ClinicalTrials.gov (via} clinicaltrials.gov)

\section{ICTRP (via the WHO platform)}

\section{Search 1}

( rhinosinusitis OR CRS OR CRSSNP OR CRSWNP OR rhinopolypy) AND ( biologics OR biologic OR biotherapy OR Interleukins OR interleukin OR IgE OR immunoglobulin OR Antiglobulin OR antiidiotype OR $\mathrm{mAB} O R$ mepo OR "IL-4" OR "IL-5" OR M1 OR "CCR4 LFA-1" OR "IL-13" OR "IL-4a" OR Dupilumab OR Reslizumab OR Benralizumab OR Mepolizumab OR Omalizumab OR Quilizumab OR Ligelizumab OR Mogamulizumab OR Efalizumab OR AMG317 OR Pitrakinra OR Lebrikizumab OR Tralokinumab OR GATA-3 OR siglec OR AK001 OR OX40L OR TNF OR TSLP OR CSL311 OR "IL-3" OR GM-CSF OR "IL-25" OR "IL-5" OR granulocyte-macrophage

\section{Search 1}

Rhinosinusitis AND Biologic* OR Rhinosinusitis AND biotherap* OR Rhinosinusitis AND Interleukin* OR Rhinosinusitis AND IgE OR Rhinosinusitis AND immunoglobulin OR Rhinosinusitis AND Antiglobulin OR Rhinosinusitis AND antiidiotype OR Rhinosinusitis AND mAB OR Rhinosinusitis AND mepo OR Rhinosinusitis AND IL OR Rhinosinusitis AND Dupilumab OR Rhinosinusitis AND Reslizumab OR Rhinosinusitis AND Benralizumab OR Rhinosinusitis AND Mepolizumab OR Rhinosinusitis AND Omalizumab OR Rhinosinusitis AND Rhinosinusitis AND Quilizumab OR Rhinosinusitis AND Ligelizumab OR Rhinosinusitis AND Mogamulizumab OR Rhinosinusitis AND Efalizumab

\section{ClinicalTrials.gov and ICTRP (via CRS)}

1 rhinosinusitis or nasosinusitis or pansinusitis or ethmoiditis or sphenoiditis AND CENTRAL:TARGET

2 kartagener ${ }^{\star}$ near syndrome* AND CENTRAL:TARGET

3 inflamm* and sinus AND CENTRAL:TARGET

4 (maxilla* or frontal $^{*}$ ) and sinus* AND CENTRAL:TARGET

5 CRSsNP or sinusitis or rhinitis or rhinopolyp* or CRSWNP AND CENTRAL:TARGET

6 (nose or nasal or rhino* or rhinitis or sinus* or sinonasal) and (papilloma* or polyp ${ }^{\star}$ ) AND CENTRAL:TARGET

7 \#1 OR \#2 OR \#3 OR \#4 OR \#5 OR \#6 AND CENTRAL:TARGET

8 (Antibod* and monoclonal):AB,E$\mathrm{H}, \mathrm{KW}, \mathrm{KY}, \mathrm{MC}, \mathrm{MH}, \mathrm{TI}, \mathrm{TO}$ AND CENTRAL:TARGET

9 (Interleukin* or IgE or immunoglobulin or Antiglobulin* 
(Continued)

\#10 TOPIC: ((nose or nasal or rhino* or rhinitis or sinus* or sinonasal) NEAR/3 (papilloma* or polyp*))

\#11 TOPIC: (rhinopolyp* or CRSwNP)

\#12 \#11 OR \#10 OR \#9 OR \#8 OR \#7

\#13 TOPIC: (Antibod* NEAR/3 monoclonal)

\#14 TOPIC: (Interleukin* or IgE or "immunoglobulin E" or Antiglobulin* or antiidiotyp ${ }^{\star}$ )

\#15 TOPIC: (anti NEAR/3 (globulin* or idiotyp* or immunoglobulin* or M1 or CCR4 or "LFA 1" or "GATA 3" or OX40L))

\#16 TOPIC: (ralokimumab or Adalimumab or Alemtuzumab or Bevacizumab or Certolizumab or Cetuximab or Denosumab or Ipilimumab or Natalizumab or Omalizumab or Palivizumab or Ranibizumab or Trastuzumab or stekinumab or mepolizumab or Nucala or SB240563 or "SB 240563" or dupilumab or REGN668 or AMG317 or "AMG 317" or AMG827 or "AMG 827" or DNAzyme or antiTSLP or CSL311 or "CSL 311" or "AMG 761" or AMG761 or "AMG 837" or KW0761 or "KW 0761" or "CSF 2" or "CSF GM")

\#17 TOPIC: (siliq or D2E7 or humira or campath or Lemtrada or avastin or cimzia or CDP870 or "CDP 870" or Erbitux or C225 or Xgeva or prolia or "AMG 162" or AMG162 or Yervoy or Tysabri or Antegren or Xolair or Synagis or RhuFab or lucentis or Herceptin or stelara or CNTO or ASM8 or granulocyte-macrophage or GM-CSF or QGE031 or Raptiva or AK001 or "AK $\left.001^{\prime \prime}\right)$

\#18 TOPIC: ((B-cell or T-cell or Eosinophil or "mast cell" or stimulating) NEAR/3 factor)

\#19 TOPIC: (CD23 or CD2 or CD11a or CD20 or CD25 opr CD252 or (receptor ${ }^{\star}$ NEAR/3 apsilon))

\#20 TOPIC: (CD NEAR/3 ("23" or antigen * or "2" or 11 a or "20" or "25" or "252"))

\#21 TOPIC: ((antigamma or "anti gamma") NEAR/3 Antibod*)
OR monoclonal AND antibodies )

\section{Search 2}

(rhinitis OR sinusitis ) AND ( recurrence OR recurrent OR chronic OR persistant OR persistance ) AND ( biologics OR biologic OR biotherapy OR Interleukins OR interleukin OR IgE OR immunoglobulin OR Antiglobulin OR antiidiotype OR mAB OR mepo OR "IL-4" OR "IL-5" OR M1 OR "CCR4 LFA-1" OR "IL-13" OR "IL-4a" OR Dupilumab OR Reslizumab OR Benralizumab OR Mepolizumab OR Omalizumab OR Quilizumab OR Ligelizumab OR Mogamulizumab OR Efalizumab OR AMG317 OR Pitrakinra OR Lebrikizumab OR Tralokinumab OR GATA-3 OR siglec OR AK001 OR OX40L OR TNF OR TSLP OR CSL311 OR "IL-3" OR GM-CSF OR "IL-25" OR "IL-5" OR granulocyte-macrophage OR monoclonal AND antibodies )

\section{Search 3}

( nose OR nasal OR sinus OR sinonasal ) AND ( polyp OR polyps) AND (biologics OR biologic OR biotherapy OR Interleukins OR interleukin OR IgE OR immunoglobulin OR Antiglobulin OR antiidiotype OR $\mathrm{mAB}$ OR mepo OR "IL-4" OR "IL-5" OR M1 OR "CCR4 LFA-1" OR "IL-13" OR "IL-4a" OR Dupilumab OR Reslizumab OR Benralizumab OR Mepolizumab OR Omalizumab OR Quilizumab OR Ligelizumab OR Mogamulizumab OR Efalizumab OR AMG317 OR Pitrakinra OR Lebrikizumab OR Tralokinumab OR GATA-3 OR siglec OR AK001 OR OX40L OR
OR Rhinosinusitis AND Pitrakinra OR Rhinosinusitis AND Lebrikizumab OR Rhinosinusitis AND Tralokinumab OR Rhinosinusitis AND siglec OR Rhinosinusitis AND monoclonal AND antibod*

\section{Search 2}

Sinusitis AND chronic AND Biologic* OR Sinusitis AND chronic AND biotherap* OR Sinusitis AND chronic AND Interleukin* OR Sinusitis AND chronic AND IgE OR Sinusitis AND chronic AND immunoglobulin OR Sinusitis AND chronic AND Antiglobulin OR Sinusitis AND chronic AND antiidiotype OR Sinusitis AND chronic AND mAB OR Sinusitis AND chronic AND mepo OR Sinusitis AND chronic AND IL OR Sinusitis AND chronic AND Dupilumab OR Sinusitis AND chronic AND Reslizumab OR Sinusitis AND chronic AND Benralizumab OR Sinusitis AND chronic AND Mepolizumab OR Sinusitis AND chronic AND Omalizumab OR Sinusitis AND chronic AND Sinusitis AND chronic AND Quilizumab OR Sinusitis AND chronic AND Ligelizumab OR Sinusitis AND chronic AND Mogamulizumab OR Sinusitis AND chronic AND Efalizumab OR Sinusitis AND chronic AND Pitrakinra OR Sinusitis AND chronic AND Lebrikizumab OR Sinusitis AND chronic AND Tralokinumab OR Sinusitis AND chronic AND siglec OR Sinusitis AND chronic AND monoclonal AND antibod*sitis AND siglec OR Sinusitis AND monoclonal AND antibod*

\section{Search 3}

or antiidiotyp $\left.{ }^{\star}\right): A B, E H, K W, K Y, M-$ C,MH,TI,TO AND CENTRAL:TARGET

10 (anti adj3 (globulin* or idiotyp* or immunoglobulin* or M1 or CCR4 or "LFA 1" or "GATA 3" or OX40L)):AB,EH,KW,KY,MC,MH,TI,TO AND CENTRAL:TARGET

11 (ralokimumab or Adalimumab or Alemtuzumab or Bevacizumab or Certolizumab or Cetuximab or Denosumab or Ipilimumab or Natalizumab or Omalizumab or Palivizumab or Ranibizumab or Trastuzumab or stekinumab or mepolizumab or Nucala or SB240563 or "SB 240563" or dupilumab or REGN668 or AMG317 or "AMG 317 " or AMG827 or "AMG 827 " or DNAzyme or antiTSLP or CSL311 or "CSL 311" or "AMG 761 " or AMG761 or "AMG 837" or KW0761 or "KW 0761" or "CSF 2" or "CSF GM"):AB,EH,KW,KY,MC,MH,TI,TO AND CENTRAL:TARGET

12 (siliq or D2E7 or humira or campath or Lemtrada or avastin or cimzia or CDP870 or "CDP 870" or Erbitux or C225 or Xgeva or prolia or "AMG 162" or AMG162 or Yervoy or Tysabri or Antegren or Xolair or Synagis or RhuFab or lucentis or Herceptin or stelara or CNTO or ASM8 or granulocyte-macrophage or GM-CSF or QGE031 or Raptiva or AK001 or "AK 001"):AB,E$\mathrm{H}$,KW, KY,MC,MH,TI,TO AND CENTRAL:TARGET

13 ((B-cell or T-cell or Eosinophil or "mast cell" or stimulating) adj3 factor):AB, EH, KW, KY,MC,MH,TI,TO AND CENTRAL:TARGET

14 (CD23 or CD2 or CD11a or CD20 or CD25 opr CD252 or (receptor* adj3 apsilon)):AB,EH, KW, KY,MC,MH,TI,TO AND CENTRAL:TARGET

15 (CD adj3 ("23" or antigen* or "2" or 11a or "20" or "25" or "252")):AB,EH, KW, KY,MC,MH,TI,TO AND CENTRAL:TARGET

16 ((antigamma or "anti gamma") and Antibod*):AB,EH,KW,KY,MC,MH,TI,TO AND CENTRAL:TARGET

17 (IgEid or "55700" or SCH55700 or CEP38072 or "CEP 38072" or 
(Continued)

835 " or GM-CSF or TNF or TSLP or OX40L)

\#23 TOPIC: (IL NEAR/3 ("5" or five or "4" or four or "13" or thirteen or " 1 " or one or "10" or ten or "11" or eleven or "12" or twelve or "15" or fifteen or "16" or sixteen or "17" or seventeen or "18" or eighteen or "2" or two or "23" or "twenty three" or "12" or twelve or "27" or "twenty seven" or "3" or three or "33" or "thirty three" or "6" or six or "7" or seven or "8" or eight or "9" or nine or 1R1 or 12p40 or IL-23p40 or 17A or 17RA or "22" or "twenty two" or "31" or "thirty one" or 31R))

\#24 TOPIC: (IL5 or IL4 or IL13 or IL1 or IL10 or IL11 or IL12 or IL15 or IL15 or IL16 or IL17 or IL18 or IL2 or IL23 or IL12 or IL27 or IL3 or IL33 or IL6 or IL7 or IL8 or IL9 or IL22 or IL31 or "IL 4R*" or "IL 5R*")

\#25 TOPIC: (biologic or biologics or biotherap *)

\#26 TOPIC: (biologic* NEAR/3 ther$\left.a p^{\star}\right)$

\#27 TOPIC: (mAB or mepo or MDX or MEDI or siglec * or "lectin 8")

\#28 TOPIC: (SAR231893 or reslizumab or siglec 8 or benralizumab or lebrikizumab or brodalumab or Tralokinumab or Quilizumab or Ligelizumab or Mogamulizumab or Efalizumab or Pitrakinra or Odulimomab or Mogamulizumabor or BCGF or binetrakin or "anti antibod*")

\#29 TOPIC: (Canakinumab or llaris or Rilonacept or Arcalyst or Anakinra or Kineret or Antril or Altrakincept or Nuvance or Pascolizumab or SB 240683 or VAK694 or QBX258 or VAK 694 or VAK-694 or dectrekumab QAX-576 or QAX576 or QAX 576 or aerovant or AER-001 or AER001 or "AER 001" or BAY-16-9996 or BAY 16-9996 or Bosatria or Nucala or CDP 835 or CDP835 or CDP-835 or CINQAIR or CTx55700 or CTx 55700 or CTX-55700 or DCP 835 or DCP-835 or DCP835 or SCH5570 or SCH 5570 SCH-5570 or TRFK-5 or TRFK 5 or TRFK 5 or BIW- $8405^{\star}$ or BIW8405* or BIW 8405* or KHK 4563 or KHK-4563 or KHK4563 or Enokizumab or 7F3com-2H2 or Ustek-
OR "IL-3" OR GM-CSF OR "IL-25" OR "IL-5" OR granulocyte-macrophage OR monoclonal AND antibodies )

Biologics for chronic rhinosinusitis (Review)

Nasal AND polyp* AND Biologic* OR Nasal AND polyp* AND biotherap* OR Nasal AND polyp* AND Interleukin * OR Nasal AND polyp* AND IgE OR Nasal AND polyp* AND immunoglobulin OR Nasal AND polyp* AND Antiglobulin OR Nasal AND polyp* AND antiidiotype OR Nasal AND polyp* AND mAB OR Nasal AND polyp* AND mepo OR Nasal AND polyp* AND IL OR Nasal AND polyp* AND Dupilumab OR Nasal AND polyp* AND Reslizumab OR Nasal AND polyp* AND Benralizumab OR Nasal AND polyp* AND Mepolizumab OR Nasal AND polyp* AND Omalizumab OR Nasal AND polyp* AND Nasal AND polyp* AND Quilizumab OR Nasal AND polyp* AND Ligelizumab OR Nasal AND polyp* AND Mogamulizumab OR Nasal AND polyp* AND Efalizumab OR Nasal AND polyp* AND Pitrakinra OR Nasal AND polyp* AND Lebrikizumab OR Nasal AND polyp* AND Tralokinumab OR Nasal AND polyp* AND siglec OR Nasal AND polyp* AND monoclonal AND antibod* $^{*}$

\section{Search 4}

Rhinitis AND chronic AND Biologic* OR Rhinitis AND chronic AND biotherap* OR Rhinitis AND chronic AND Interleukin* OR Rhinitis AND chronic AND IgE OR Rhinitis AND chronic AND immunoglobulin OR Rhinitis AND chronic AND Antiglobulin OR Rhinitis AND chronic AND antiidiotype OR Rhinitis AND chronic AND mAB OR Rhinitis AND chronic AND mepo OR Rhinitis AND chronic ANDII cinqair or DCP835 or "DCP 835" or GM-CSF or TNF or TSLP or OX40L):AB,EH,KW,KY,MC,MH,TI,TO AND CENTRAL:TARGET

18 (IL adj3 ("5" or five or "4" or four or "13" or thirteen or " 1 " or one or "10" or ten or "11" or eleven or "12" or twelve or "15" or fifteen or "16" or sixteen or "17" or seventeen or "18" or eighteen or "2" or two or "23" or "twenty three" or "12" or twelve or "27" or "twenty seven" or "3" or three or "33" or "thirty three" or "6" or six or "7" or seven or "8" or eight or "9" or nine or $5 R^{\star}$ or $1 R 1$ or $4 R^{\star}$ or $12 p 40$ or IL-23p40 or 17A or 17RA or "22" or "twenty two" or "31" or "thirty one" or 31R)):AB,EH,KW,KY,MC,MH,TI,TO AND CENTRAL:TARGET

19 (biologic or biologics or biother$\mathrm{ap}^{\star}$ ):AB,EH,KW, KY,MC,MH,TI,TO AND CENTRAL:TARGET

20 biologic ${ }^{\star}$ adj3 therap * AND CENTRAL:TARGET

21 ( $\mathrm{mAB}$ or mepo or MDX or MEDI or siglec* or "lectin 8"):AB,EH,KW, KY,MC,MH,TI,TO AND CENTRAL:TARGET

22 SAR231893 or reslizumab or siglec8 or benralizumab or lebrikizumab or brodalumab or Tralokinumab or Quilizumab or Ligelizumab or Mogamulizumab or Efalizumab or Pitrakinra or Odulimomab or Mogamulizumabor or BCGF or binetrakin or "anti antibod*" AND CENTRAL:TARGET

23 (Canakinumab or Ilaris or Rilonacept or Arcalyst or Anakinra or Kineret or Antril or Altrakincept or Nuvance or Pascolizumab or SB 240683 or VAK694 or QBX258 or VAK 694 or VAK-694 or dectrekumab QAX-576 or QAX576 or QAX 576 or aerovant or AER-001 or AER001 or "AER 001" or BAY-16-9996 or BAY 16-9996 or Bosatria or Nucala or CDP 835 or CDP835 or CDP-835 or CINQAIR or CTx55700 or CTx 55700 or CTx-55700 or DCP 835 or DCP-835 or DCP835 or SCH5570 or SCH 5570 SCH-5570 or TRFK-5 or TRFK 5 or TRFK5 or BIW-8405* or BIW8405* or BIW $8405^{\star}$ or KHK 4563 or KHK-4563 or KHK4563 or 
(Continued)

inumab or Stelara or CNTO-1275 or CNTO1275 or CNTO 1275 or Anrukinzuma* or IMA-638 or PF-05230917 or GSK679586 or GSK-679586 or GDK 679586 or IMA026 or IMA-026 or "IMA 026" or IMA638 or IMA 638 MILR1444A or MILR 1444A or MILR-1444A or PRO-301444 or PRO301444 or PRO 301444 or RG-3637 or RG3637 or RG 3637 or RO-5490255 or RO5490255 or RO 5490255 or TNX-650 or TNX650 or TNX 650 or RPC-4046 or ABT-308 or RPC 4046 or ABT308 or RPC 4046 or ABT 308 or CAT-354 or CAT354 or CAT 354 or Secukinumab or Cosentyx or AIN-457 or KB-03303A or NVP-AIN 457 or AIN457 or KB03303A or NVPAIN457 or AIN 457 or KB $03303 A$ or NVP-AIN-457 or KHK-4827 or KHK4827 or KHK 4827 or fezakinumab * or ILV-094 or PF-5212367 or ILV094 or PF5212367 or "ILV 094" or PF 5212367 or BMS-981164 or BMS981164 or BMS 981164 or Nemolizumab or CIM331 or CIM 331 or CIM-331 or Lenzilumab or KB003 or "KB 003" or KB-003 or ABT-D2E7 or D2E7 or LU 200134 or ABTD2E7 or LU200134 or ABT D2E7 or LU 200134 or Golimumab or Simponi or CNTO-148 or CNTO148 or CNTO 148 or Inflixima or CA2 or CenTNF or Remicade or TA-650 or TA650 or TA 650 or Etanercept or Enbrel or p75TNFR-Ig or rhu TNFR-Fc or TNFR-Fc-p75 or TNR-001 or TNR001 or "TNR 001" or AMG-157 or MEDI-9929 or AMG157 or AMG 157 MEDI4212 or MEMP1972A or RG7449 or MEMP 1972A or RG 7449 or MEMP-1972A or RG-7449 or Mogamulizumab or KM8761 or Poteligeo or KM-8761 or KM 8761 or Alefacept or Amevive or "ASP 0485" or BG 9273 or BG 9712 or ASP0485 or BG9273 or BG9712 or ASP-0485 or BG-9273 or BG-9712 or Xanelim or Rituximab or Rituxan or Daclizumab or Zenapax or Oxeluma* or huMAb or OX40L or RG 4930 or RO4989991 or RG4930 or RG-4930 or RO 4989991 or RO-4989991 or Bertilimumab or Tezepeluma or Isunakinra or "Fusion Protein*" or cytokine*)

\#30 \#29 OR \#28 OR \#27 OR \#26 OR \#25 OR \#24 OR \#23 OR \#22 OR \#21 OR \#20 OR \#19 OR \#18 OR \#17 OR \#16 OR \#15 OR \#14 OR \#13

\#31 \#30 AND \#12
OR Rhinitis AND chronic AND Dupilumab OR Rhinitis AND chronic AND Reslizumab OR Rhinitis AND chronic AND Benralizumab OR Rhinitis AND chronic AND Mepolizumab OR Rhinitis AND chronic AND Omalizumab OR Rhinitis AND chronic AND Rhinitis AND chronic AND Quilizumab OR Rhinitis AND chronic AND Ligelizumab OR Rhinitis AND chronic AND Mogamulizumab OR Rhinitis AND chronic AND Efalizumab OR Rhinitis AND chronic AND Pitrakinra OR Rhinitis AND chronic AND Lebrikizumab OR Rhinitis AND chronic AND Tralokinumab OR Rhinitis AND chronic AND siglec OR Rhinitis AND chronic AND monoclonal AND antibod* $^{*}$
Enokizumab or $7 \mathrm{~F} 3 \mathrm{com}-2 \mathrm{H} 2$ or Ustekinumab or Stelara or CNTO-1275 or CNTO1275 or CNTO 1275 or Anrukinzuma* or IMA-638 or PF-05230917 or GSK679586 or GSK-679586 or GDK 679586 or IMA026 or IMA-026 or "IMA 026" or IMA638 or IMA 638 MILR1444A or MILR 1444A or MILR-1444A or PRO-301444 or PRO301444 or PRO 301444 or RG-3637 or RG3637 or RG 3637 or RO-5490255 or RO5490255 or RO 5490255 or TNX-650 or TNX650 or TNX 650 or RPC-4046 or ABT-308 or RPC 4046 or ABT308 or RPC 4046 or ABT 308 or CAT-354 or CAT354 or CAT 354 or Secukinumab or Cosentyx or AIN-457 or KB-03303A or NVP-AIN 457 or AIN457 or KB03303A or NVPAIN457 or AIN 457 or KB $03303 \mathrm{~A}$ or NVP-AIN-457 or KHK-4827 or KHK4827 or KHK 4827 or fezakinumab * or ILV-094 or PF-5212367 or ILV094 or PF5212367 or "ILV 094" or PF 5212367 or BMS-981164 or BMS981164 or BMS 981164 or Nemolizumab or CIM331 or CIM 331 or CIM-331 or Lenzilumab or KB003 or "KB 003 " or KB-003 or ABT-D2E7 or D2E7 or LU 200134 or ABTD2E7 or LU200134 or ABT D2E7 or LU 200134 or Golimumab or Simponi or CNTO-148 or CNTO148 or CNTO 148 or Inflixima or CA2 or CenTNF or Remicade or TA-650 or TA650 or TA 650 or Etanercept or Enbrel or p75TNFR-Ig or rhu TNFR-FC or TNFR-FCp75 or TNR-001 or TNR001 or "TNR 001" or AMG-157 or MEDI-9929 or AMG157 or AMG 157 MEDI4212 or MEMP1972A or RG7449 or MEMP 1972A or RG 7449 or MEMP-1972A or RG-7449 or Mogamulizumab or KM8761 or Poteligeo or KM-8761 or KM 8761 or Alefacept or Amevive or "ASP 0485 " or BG 9273 or BG 9712 or ASP0485 or BG9273 or BG9712 or ASP-0485 or BG-9273 or BG-9712 or Xanelim or Rituximab or Rituxan or Daclizumab or Zenapax or Oxeluma* or huMAb or OX40L or RG 4930 or RO4989991 or RG4930 or RG-4930 or RO 4989991 or RO-4989991 or Bertilimumab or Tezepeluma or Isunakinra or "Fusion Protein ${ }^{\star}$ or cytokine*):AB,E$\mathrm{H}, \mathrm{KW}, \mathrm{KY}, \mathrm{MC}, \mathrm{MH}, \mathrm{TI}, \mathrm{TO}$ AND CENTRAL:TARGET 
(Continued)

\#32 TOPIC: ((randomised OR randomized OR randomisation OR randomisation OR placebo* OR (random* AND (allocat* OR assign*)) OR (blind * AND (single OR double OR treble OR triple))))

\#33 \#32 AND \#31
24 (IL5 or IL4 or IL13 or IL1 or IL10 or IL11 or IL12 or IL15 or IL15 or IL16 or IL17 or IL18 or IL2 or IL23 or IL12 or IL27 or IL3 or IL33 or IL6 or IL7 or IL8 or IL9 or IL22 or IL31):AB,EH,KW,KY,MC,MH,TI,TO AND CENTRAL:TARGET

25 \#24 OR \#23 OR \#22 OR \#21 OR \#20 OR \#19 OR \#18 OR \#17 OR \#16 OR \#15 OR \#14 OR \#13 OR \#12 OR \#11 OR \#10 OR \#9 OR \#8

26 \#25 AND \#7

27 nct:AU OR http*:SO AND CENTRAL:TARGET

28 \#26 AND \#27

\section{Appendix 2. Data extraction form}

REF ID: $\quad$ Study title:

Date of extraction: $\quad$ Extracted by:

General comments/notes (internal for discussion):

Group A (Interven- Group B (Compartion) ison)

No. of people screened

No. of participants randomised - all

No. randomised to each group

No. receiving treatment as allocated

No. not receiving treatment as allocated

- Reason 1

- Reason 2

Ne_drannad Lut

Biologics for chronic rhinosinusitis (Review)

Copyright (c) 2020 The Cochrane Collaboration. Published by John Wiley \& Sons, Ltd. 
(Continued)

(no follow-up data for any outcome available)

No. excluded from analysis ${ }^{1}$ (for all outcomes)

- Reason 1

- Reason 2

Number analysed

1This should be the people who received the treatment and were therefore not considered 'dropouts' but were excluded from all analyses (e.g. because the data could not be interpreted or the outcome was not recorded for some reason).

\section{Information to go into 'Characteristics of included studies' table}

Methods $\quad \mathrm{X}$ arm, double/single/non-blinded, [multicentre] parallel-group/cross-over/cluster-RCT, with $\mathrm{x}$ du-
ration of treatment and $x$ duration of follow-up

Participants Location: country, no of sites etc.

\section{Setting of recruitment and treatment:}

\section{Sample size:}

- Number randomised: $x$ in intervention, $y$ in comparison

- Number completed: $x$ in intervention, $y$ in comparison

Participant (baseline) characteristics:

- Age:

- Gender:

- Main diagnosis: [as stated in paper]

- Polyps status: $\mathrm{x} \%$ with polyps/no information [add info on mean polyps score if available]

- Previous sinus surgery status: [x\% with previous surgery]

- Previous courses of steroids: [add info on mean number of courses if available

Other important effect modifiers, if applicable (e.g. aspirin sensitivity, comorbidities of asthma):

Inclusion criteria: [state diagnostic criteria used for CRS, polyps score if available] Exclusion criteria:

\section{Interventions}

Intervention $(\mathbf{n}=\mathbf{x})$ : drug name, method of administration, dose per day/frequency of administration, duration of treatment

\section{Comparator group $(n=y)$ :}

\section{Use of additional interventions (common to both treatment arms):}

\section{Outcomes}

\section{Outcomes of interest in the review:}

Primary outcomes:

- Health-related quality of life, disease-specific

- Disease severity symptom score

- Significant adverse effects: local reaction at the injection site, including swelling, redness

Secondary outcomes: 
- Health-related quality of life, generic

- Nasopharyngitis, including sore throat

- Endoscopy (polyps size or overall score)

- CT scan

\section{Funding sources}

\section{Declarations of interest}

'No information provided'/'None declared'/State source of funding

'No information provided'/'None declared'/State conflict

\section{Notes}

\begin{tabular}{|c|c|c|}
\hline Bias (ROB 1.0) & $\begin{array}{l}\text { Authors' judge- } \\
\text { ment }\end{array}$ & Support for judgement \\
\hline \multirow[t]{2}{*}{ Random sequence generation (selection bias) } & & Quote: "..." \\
\hline & & Comment: \\
\hline \multirow[t]{2}{*}{ Allocation concealment (selection bias) } & & Quote: "..." \\
\hline & & Comment: \\
\hline \multirow[t]{2}{*}{ Blinding of participants and personnel (performance bias) } & & Quote: "..." \\
\hline & & Comment: \\
\hline \multirow[t]{2}{*}{ Blinding of outcome assessment (detection bias) } & & Quote: "..." \\
\hline & & Comment: \\
\hline \multirow[t]{2}{*}{ Incomplete outcome data (attrition bias) } & & Quote: "..." \\
\hline & & Comment: \\
\hline \multirow[t]{2}{*}{ Selective reporting (reporting bias) } & & Quote: "..." \\
\hline & & Comment: \\
\hline Other bias (see section 8.15) & & Quote: "..." \\
\hline Insensitive/non-validated instrument? & & Comment: \\
\hline
\end{tabular}




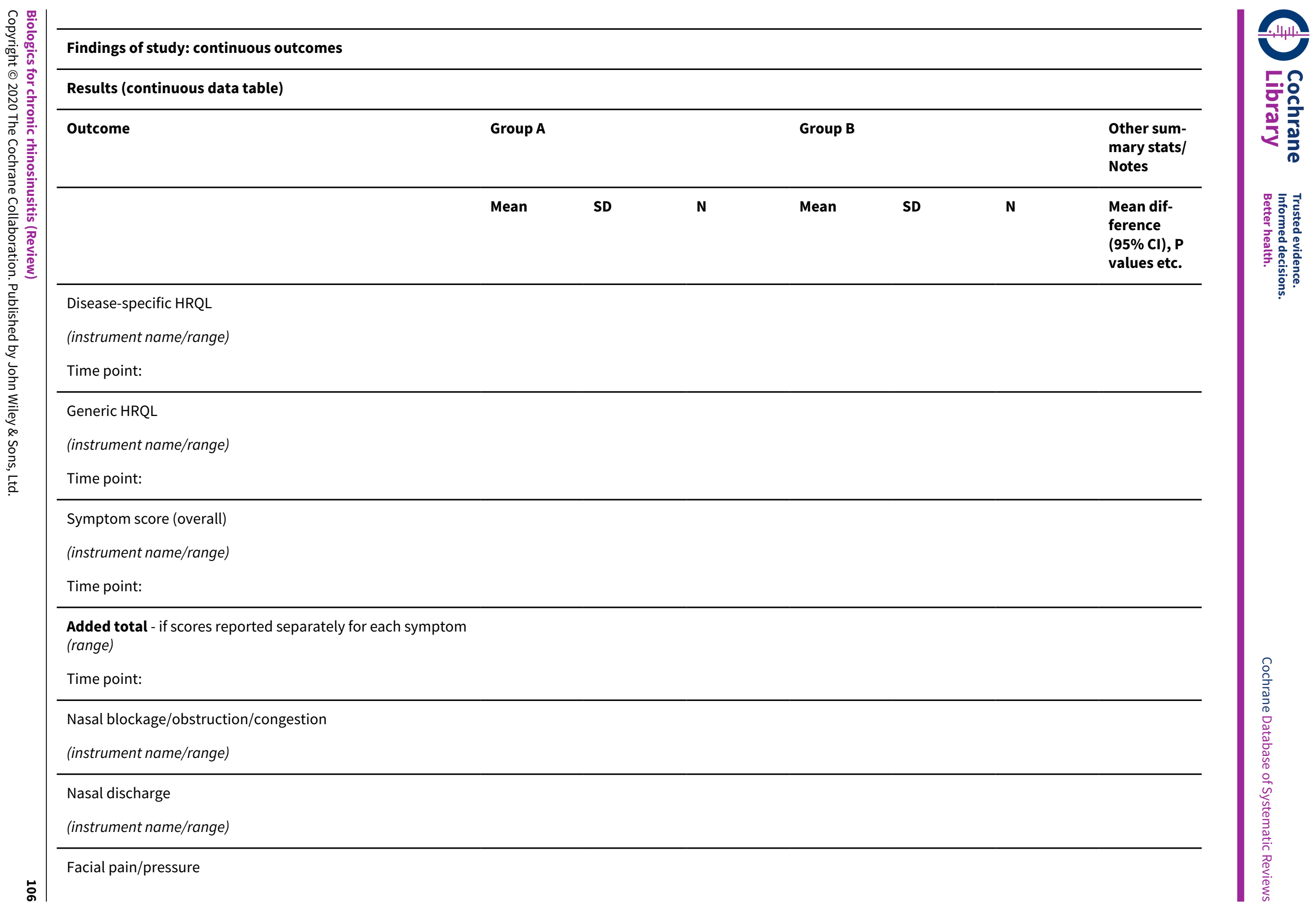




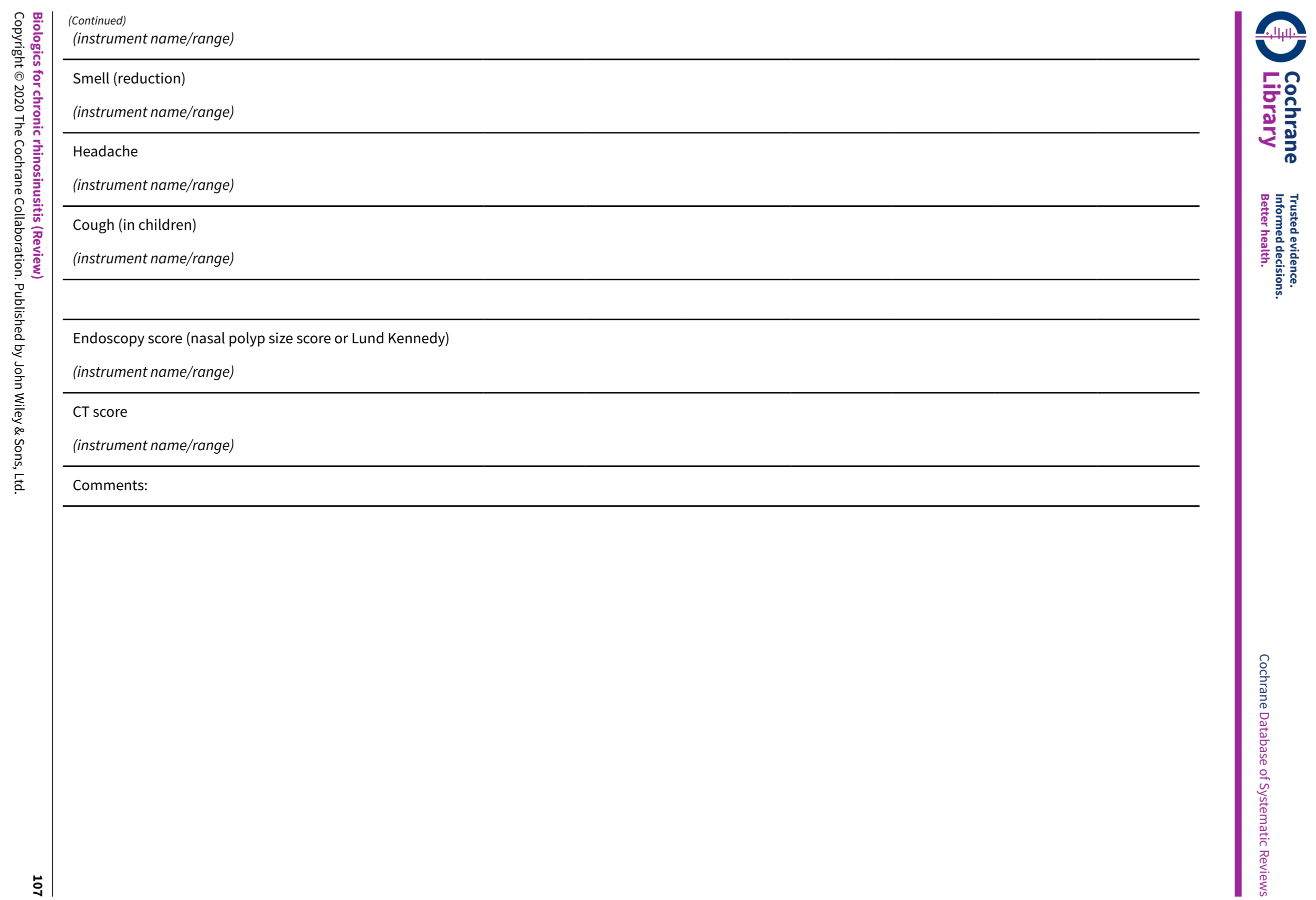




\section{Results (dichotomous data table)}

\begin{tabular}{|c|c|c|c|c|c|}
\hline \multirow[t]{2}{*}{ Outcome } & \multicolumn{2}{|l|}{ Group A } & \multicolumn{2}{|l|}{ Group B } & \multirow{2}{*}{$\begin{array}{l}\text { Other summary stats/ } \\
\text { notes } \\
\text { P values, RR }(95 \% \mathrm{CI}) \text {, } \\
\text { OR }(95 \% \mathrm{CI})\end{array}$} \\
\hline & $\begin{array}{l}\text { No. of } \\
\text { people } \\
\text { with } \\
\text { events }\end{array}$ & $\begin{array}{l}\text { No. of } \\
\text { people } \\
\text { analysed }\end{array}$ & $\begin{array}{l}\text { No. of } \\
\text { people } \\
\text { with } \\
\text { events }\end{array}$ & $\begin{array}{l}\text { No. of } \\
\text { people } \\
\text { analysed }\end{array}$ & \\
\hline
\end{tabular}

Local reaction at the injection site, including

swelling, redness

Nasopharyngitis, including sore throat

Comments:

Appendix 3. Search strategies for Clinical Study Reports

\begin{tabular}{|c|c|c|c|}
\hline EUCTR & Novartis (searched via Google) & $\begin{array}{l}\text { GlaxoSmithKlein (searched } \\
\text { via Google) }\end{array}$ & Other \\
\hline $\begin{array}{l}\text { ( rhinosinusitis OR CRS OR CRSs- } \\
\text { NP OR CRSwNP OR rhinopolypy) } \\
\text { AND ( biologics OR biologic OR } \\
\text { biotherapy OR Interleukins OR in- } \\
\text { terleukin OR IgE OR immunoglob- } \\
\text { ulin OR Antiglobulin OR anti- } \\
\text { idiotype OR mAB OR mepo OR } \\
\text { "IL-4" OR "IL-5" OR M1 OR "CCR4 } \\
\text { LFA-1" OR "IL-13" OR "IL-4a" OR } \\
\text { Dupilumab OR Reslizumab OR } \\
\text { Benralizumab OR Mepolizumab } \\
\text { OR Omalizumab OR Quilizum- } \\
\text { ab OR Ligelizumab OR Moga- } \\
\text { mulizumab OR Efalizumab OR } \\
\text { AMG317 OR Pitrakinra OR Le- } \\
\text { brikizumab OR Tralokinumab } \\
\text { OR GATA-3 OR siglec OR AKO01 } \\
\text { OR OX40L OR TNF OR TSLP OR } \\
\text { CSL311 OR "IL-3" OR GM-CSF OR } \\
\text { "IL-25" OR "IL-5" OR granulo- } \\
\text { cyte-macrophage OR "monoclon- } \\
\text { al antibodies" ) } \\
\text { ( rhinitis OR sinusitis ) AND ( re- } \\
\text { currence OR recurrent OR chron- } \\
\text { ic OR persistant OR persistance ) } \\
\text { AND ( biologics OR biologic OR } \\
\text { biotherapy OR Interleukins OR in- } \\
\text { terleukin OR IgE OR immunoglob- } \\
\text { ulin OR Antiglobulin OR anti- } \\
\text { idiotype OR mAB OR mepo OR } \\
\text { "IL-4" OR "IL-5" OR M1 OR "CCR4 }\end{array}$ & $\begin{array}{l}\text { site:novctrd.com ( rhinosinusitis OR CRS } \\
\text { OR CRSsNP OR CRSwNP OR rhinopolypy) } \\
\text { ( biologics OR biologic OR biotherapy OR } \\
\text { Interleukins OR interleukin OR IgE OR im- } \\
\text { munoglobulin OR Antiglobulin OR anti- } \\
\text { idiotype OR mAB OR mepo OR "IL-4" OR } \\
\text { "IL-5" OR M1 OR "CCR4 LFA-1" OR "IL-13" OR } \\
\text { "IL-4a" OR Dupilumab OR Reslizumab OR } \\
\text { Benralizumab OR Mepolizumab) } \\
\text { site:novctrd.com ( rhinosinusitis OR CRS OR } \\
\text { CRSsNP OR CRSwNP OR rhinopolypy) (Oma- } \\
\text { lizumab OR Quilizumab OR Ligelizumab OR } \\
\text { Mogamulizumab OR Efalizumab OR AMG317 } \\
\text { OR Pitrakinra OR Lebrikizumab OR Tralok- } \\
\text { inumab OR GATA-3 OR siglec OR AKO01 OR } \\
\text { OX40L OR TNF OR TSLP OR CSL311 OR "IL-3" } \\
\text { OR GM-CSF OR "IL-25" OR "IL-5" OR granulo- } \\
\text { cyte-macrophage) } \\
\text { site:novctrd.com ( rhinosinusitis OR CRS OR } \\
\text { CRSsNP OR CRSwNP OR rhinopolypy) (mon- } \\
\text { oclonal AND antibodies) } \\
\text { site:novctrd.com ( rhinitis OR sinusitis) ( re- } \\
\text { currence OR recurrent OR chronic OR per- } \\
\text { sistant OR persistance) ( biologics OR bio- } \\
\text { logic OR biotherapy OR Interleukins OR in- } \\
\text { terleukin OR IgE OR immunoglobulin OR } \\
\text { Antiglobulin OR antiidiotype OR mAB OR } \\
\text { mepo OR "IL-4" OR "IL-5" OR M1 OR "CCR4 } \\
\text { LFA-1" OR "IL-13" OR "IL-4a" OR Dupilumab } \\
\text { OR Reslizumab) }\end{array}$ & $\begin{array}{l}\text { site:gsk-studyregister.com ( rhi- } \\
\text { nosinusitis OR CRS OR CRSsNP } \\
\text { OR CRSwNP OR rhinopolypy) } \\
\text { ( biologics OR biologic OR } \\
\text { biotherapy OR Interleukins } \\
\text { OR interleukin OR IgE OR im- } \\
\text { munoglobulin OR Antiglobu- } \\
\text { lin OR antiidiotype OR mAB OR } \\
\text { mepo OR "IL-4" OR "IL-5" OR } \\
\text { M1 OR "CCR4 LFA-1" OR "IL-13" } \\
\text { OR "IL-4a" OR Dupilumab OR } \\
\text { Reslizumab OR Benralizumab } \\
\text { OR Mepolizumab) } \\
\text { site:gsk-studyregister.com ( rhi- } \\
\text { nosinusitis OR CRS OR CRSsNP } \\
\text { OR CRSwNP OR rhinopolypy) } \\
\text { (Omalizumab OR Quilizum- } \\
\text { ab OR Ligelizumab OR Moga- } \\
\text { mulizumab OR Efalizumab OR } \\
\text { AMG317 OR Pitrakinra OR Le- } \\
\text { brikizumab OR Tralokinumab } \\
\text { OR GATA-3 OR siglec OR AKO01 } \\
\text { OR OX40L OR TNF OR TSLP OR } \\
\text { CSL311 OR "IL-3" OR GM-CSF OR } \\
\text { "IL-25" OR "IL-5" OR granulo- } \\
\text { cyte-macrophage) } \\
\text { site:gsk-studyregister.com ( rhi- } \\
\text { nosinusitis OR CRS OR CRSsNP } \\
\text { OR CRSwNP OR rhinopolypy) } \\
\text { (monoclonal AND antibodies) }\end{array}$ & $\begin{array}{l}\text { We down- } \\
\text { loaded } \\
\text { spreadsheet, } \\
\text { with com- } \\
\text { plete lists of } \\
\text { trials from } \\
\text { the follow- } \\
\text { ing sources, } \\
\text { and interi- } \\
\text { gated these } \\
\text { to identify } \\
\text { unique trials: } \\
\text { - GSK } \\
\text { - EMA - } \\
\text { pending } \\
\text { - EMA - ap- } \\
\text { prove }\end{array}$ \\
\hline
\end{tabular}


(Continued)

LFA-1" OR "IL-13" OR "IL-4a" OR Dupilumab OR Reslizumab OR Benralizumab OR Mepolizumab OR Omalizumab OR Quilizumab OR Ligelizumab OR Mogamulizumab OR Efalizumab OR AMG317 OR Pitrakinra OR Lebrikizumab OR Tralokinumab OR GATA-3 OR siglec OR AK001 OR OX40L OR TNF OR TSLP OR CSL311 OR "IL-3" OR GM-CSF OR "IL-25" OR "IL-5" OR granulocyte-macrophage OR "monoclonal antibodies" )

( nose OR nasal OR sinus OR sinonasal ) AND ( polyp OR polyps ) AND ( biologics OR biologic OR biotherapy OR Interleukins OR interleukin OR IgE OR immunoglobulin OR Antiglobulin OR antiidiotype OR $\mathrm{mAB}$ OR mepo OR "IL-4" OR "IL-5" OR M1 OR "CCR4 LFA-1" OR "IL-13" OR "IL-4a" OR Dupilumab OR Reslizumab OR Benralizumab OR Mepolizumab OR Omalizumab OR Quilizumab OR Ligelizumab OR Mogamulizumab OR Efalizumab OR AMG317 OR Pitrakinra OR Lebrikizumab OR Tralokinumab OR GATA-3 OR siglec OR AK001 OR OX40L OR TNF OR TSLP OR CSL311 OR "IL-3" OR GM-CSF OR "IL-25" OR "IL-5" OR granulocyte-macrophage OR "monoclonal antibodies" ) site:novctrd.com ( rhinitis OR sinusitis) (recurrence OR recurrent OR chronic OR persistant OR persistance) (Omalizumab OR Quilizumab OR Ligelizumab OR Mogamulizumab OR Efalizumab OR AMG317 OR Pitrakinra OR Lebrikizumab OR Tralokinumab OR GATA-3 OR siglec OR AK001 OR OX40L OR TNF OR TSLP OR CSL311 OR "IL-3" OR GM-CSF OR "IL-25")

site:novctrd.com ( rhinitis OR sinusitis) (recurrence OR recurrent OR chronic OR persistant OR persistance) (Benralizumab OR Mepolizumab OR granulocyte-macrophage OR "IL-5" OR (monoclonal AND antibodies)

site:novctrd.com ( nose OR nasal OR sinus OR sinonasal ) ( polyp OR polyps) (Benralizumab OR Mepolizumab OR granulocyte-macrophage OR "IL-5" OR (monoclonal AND antibodies)

site:novctrd.com ( nose OR nasal OR sinus OR sinonasal ) ( polyp OR polyps) ( biologics OR biologic OR biotherapy OR Interleukins OR interleukin OR IgE OR immunoglobulin OR Antiglobulin OR antiidiotype OR mAB OR mepo OR "IL-4" OR "IL-5" OR M1 OR "CCR4 LFA-1" OR "IL-13" OR "IL-4a" OR Dupilumab OR Reslizumab)

site:novctrd.com ( nose OR nasal OR sinus OR sinonasal ) ( polyp OR polyps) (O1malizumab OR Quilizumab OR Ligelizumab OR Mogamulizumab OR Efalizumab OR AMG317 OR Pitrakinra OR Lebrikizumab OR Tralokinumab OR GATA-3 OR siglec OR AK001 OR OX40L OR TNF OR TSLP OR CSL311 OR "IL-3" OR GM-CSF OR "IL-25") site:gsk-studyregister.com (rhinitis OR sinusitis) (recurrence OR recurrent OR chronic OR persistant OR persistance) ( biologics OR biologic OR biotherapy OR Interleukins OR interleukin OR IgE OR immunoglobulin OR Antiglobulin OR antiidiotype OR MAB OR mepo OR "IL-4" OR "IL-5" OR M1 OR "CCR4 LFA-1" OR "IL-13" OR "IL-4a" OR Dupilumab OR Reslizumab)

site:gsk-studyregister.com ( rhinitis OR sinusitis) ( recurrence OR recurrent OR chronic OR persistant OR persistance) (Omalizumab OR Quilizumab OR Ligelizumab OR Mogamulizumab OR Efalizumab OR AMG317 OR Pitrakinra OR Lebrikizumab OR Tralokinumab OR GATA-3 OR siglec OR AK001 OR OX40L OR TNF OR TSLP OR CSL311 OR "IL-3" OR GM-CSF OR "IL-25")

site:gsk-studyregister.com ( rhinitis OR sinusitis) ( recurrence OR recurrent OR chronic OR persistant OR persistance) (Benralizumab OR Mepolizumab OR granulocyte-macrophage OR "IL-5" OR (monoclonal AND antibodies)

site:gsk-studyregister.com ( nose OR nasal OR sinus OR sinonasal) ( polyp OR polyps) (Benralizumab OR Mepolizumab OR granulocyte-macrophage OR "IL-5" OR (monoclonal AND antibodies)

site:gsk-studyregister.com ( nose OR nasal OR sinus OR sinonasal) ( polyp OR polyps) (biologics OR biologic OR biotherapy OR Interleukins OR interleukin OR IgE OR immunoglobulin OR Antiglobulin OR antiidiotype OR $\mathrm{MAB}$ OR mepo OR "IL-4" OR "IL-5" OR M1 OR "CCR4 LFA-1" OR "IL-13" OR "IL-4a" OR Dupilumab OR Reslizumab)

site:gsk-studyregister.com ( nose OR nasal OR sinus OR sinonasal ) ( polyp OR polyps) (O1malizumab OR Quilizum- 
ab OR Ligelizumab OR Mogamulizumab OR Efalizumab OR AMG317 OR Pitrakinra OR Lebrikizumab OR Tralokinumab OR GATA-3 OR siglec OR AK001 OR OX40L OR TNF OR TSLP OR CSL311 OR "IL-3" OR GM-CSF OR "IL-25")

\section{Appendix 4. Responses to requests for data}

Email from Kyowa Kirin RE: NCT02772419 (8 January 2020)

Dear Ms. Cox,

Thank you for your prompt reply.

Unfortunately, we cannot share the study date of KHK4563-005 with you.

As AstraZeneca now has global rights to Benralizumab for all current and future indication, Kyowa Kirin cannot provide study data without AstraZeneca's permission.

Please refer our Press Release on Mar. 25, 2019.

https://www.kyowakirin.com/media_center/news_releases/2019/e20190325_01.html

We appreciate it if you could wait for our paper to be published.

Best regards,

Kyowa Kirin Co., Ltd.

\section{CONTRIBUTIONSOF AUTHORS}

Lee-Yee Chong: scoped the review, and designed and wrote the protocol. Screened the search results and selected studies, carried out statistical analyses, and reviewed and edited the text of the review.

Patorn Piromchai: commented on the draft protocol and agreed the final version. Screened the search results and selected studies, carried out data checking of statistical analysis, reviewed the analyses of results and provided clinical guidance at all stages of the review, reviewed and edited the text of the review.

Steve Sharp: advised on the search strategy, commented on the draft protocol and agreed the final version. Screened the search results and selected studies. Carried out tasks related to searching for other resources.

Kornkiat Snidvongs: commented on the draft protocol and agreed the final version. Selected studies, reviewed the analyses and reviewed and edited the text of the review.

Carl Philpott: clinical guidance at all stages of the review; reviewed the analyses and reviewed and edited the text of the review.

Claire Hopkins: clinical guidance at all stages of the review; reviewed the analyses and reviewed and edited the text of the review.

Martin J Burton: clinical guidance at all stages of the review; screened the search results and selected studies, carried out data extraction, reviewed the analyses, wrote, reviewed and edited the text of the review.

\section{DECLARATIONSOF INTEREST}

Lee-Yee Chong: none known.

Patorn Piromchai: none known.

Steve Sharp: Steve Sharp's employer, the National Institute for Health and Care Excellence (NICE), has produced guidance on related topics such as sinusitis, which he has not contributed to.

Kornkiat Snidvongs: none known. 
Carl Philpott: Carl Philpott has previously received consultancy fees for Acclarent, Navigant, Aerin Medical and Entellus, and is a trustee of the patient charity Fifth Sense. He is an investigator on a clinical trial that may be included in this review, but will have no role in the data extraction, risk of bias assessment or data analysis for this study.

Claire Hopkins: Claire Hopkins has participated in advisory boards for Olympus, Chordate, Smith \& Nephew and Sanofi to provide expertise with regards to study design and outcome assessment, and interpretation of trial data. She is an investigator on a clinical trial that is included in this review, but had no role in the data extraction, risk of bias assessment or data analysis for this study (LIBERTY SINUS 24; LIBERTY SINUS 52).

Martin J Burton: Professor Martin Burton is joint Co-ordinating Editor of Cochrane ENT, but had no role in the editorial process for this review.

\section{SOURCES OF SUPPORT}

\section{Internal sources}

- No sources of support supplied

\section{External sources}

- National Institute for Health Research, UK.

Infrastructure funding for Cochrane ENT

- National Institute for Health Research, UK.

Cochrane-NIHR Incentive Award 2019

\section{DIFFERENCES BETWEEN PROTOCOL AND REVIEW}

As planned we identified completed trials that have not been published, but we did not contact the principal investigator or pharmaceutical company to obtain original data or clinical study reports, because the studies identified were not yet due to be published. We plan to make these contacts over the coming months and to incorporate any data into the next published version of this living systematic review.

\section{Clinical study reports (CSRs) and other sources of evidence}

We planned to request data from various sources beyond those listed above under electronic searches. We ran the searches as listed above and did not identify any additional reports of known trials, or trials not identified via the electronic searches. We did not, therefore, proceed to make contact but we plan to make additional efforts in this area for the first update of this living systematic review.

We did not search Clinical Study Data Request (CSDR) (https://clinicalstudydatarequest.com), AllTrials (http://www.alltrials.net) or the TrialsTracker website (https://trialstracker.ebmdatalab.net), because we determined that they were not useful for the identification of clinical study reports and other sources of evidence.

We searched the European Medicines Agency (EMEA) (http://www.emea.europa.eu), but did not make a formal request for all relevant clinical study reports (CSRs) to the European Medicines Agency (EMA) under the Access to Documents Policy (0043). We plan to pursue this as part of the planned update of this living systematic review. We did not search the UK Medicine and Healthcare Regulatory Authority (UK MHRA), as there is no database of trials to search. We plan to contact the UK MHRA to request clinical study reports for identified trials regulated by them, as part of the planned update of this living systematic review. 